\title{
Natural Gas Monthly
}

\author{
Septemiver 1990
}

\section{Energy Information Administration \\ Office of Oil and Gas \\ U.S. Department of Energy \\ Washington, DC 20585}

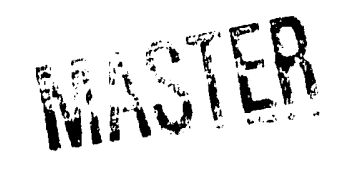

$$
\begin{aligned}
& \text { DISTRIBUTION OF THIS DOCUMENT IS UNLMMTE } \\
& \text { P2 }
\end{aligned}
$$


Energy Information Administration

Electronic Publication System (E.P(B)

User Instructions

Sclected Weekly Petroleum Status Report (WPSR), Petroleum Supply Morlhly (PSM), He'kly Coal Produtiton (WCP), Electric: Power Monthly (EPM), and Natural Gas Momhly (NGMR) statistics are now available clectronically on the Encrgy Information Administration (EIA) Computer Facility. Public acciss lo these machine-readable statistics is possible by dialing (202) $586-8658$ for 300 baud or 1200 baud line speeds. Communications are Asynchronous and require a standard ASCII-type terminal. There is no charge for this service. Although no password is required, you will be requested to use your telephone number as a user identifier. This service is available 7 days per week $(8:(0)$ a.m. - 11:(0) p.m., Monday through Friday, and 10:00 a.m.-6:(0) p.m., weekends and holidays). Weckly petroleum and coal stitistics are updated on Wednesday (Thursday in the event of a holiday) after 5:(0) p.m. Monthly petroleum supply data for the current month are also provided and are updated by 5:(X) p.m. on the 24 th of each month. Nonthly statistics from the Electric Power Monthlyare available on or about the first working day of each month. Monthly statistics from the Natural Gas Monthly are available on or about the 20) th of each month. Questions or comments on petroleum data should be directed to Dale Bodzer at (202)586-1257. Questions or comments on coal data should be directed to Noel Balthasar at (202)586-5252. Questions or comments on clectricity data should be directed to Deborah Bolden at (202)5866872. Questions or comments on natural gas data should be directed to Jim Todaro at (202) 586-6305.

Access Instructions:

1) DIAL (202) $5866 \cdot 8658$

2) HIT RETURN (CARRIAGE RETURN) TWO OR THREE TIMES UNTIL THE EPUB BANNER APPEARS.

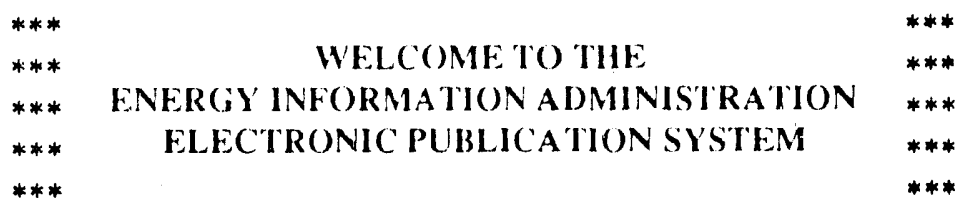

3) SELECT THE STATISTICS YOU WISH FROM THE MENU.

THE FOLLOWING REPORTS ARE AVAILABLE:

WPSR - WEEKLY PETROLEUM STATUS REPOKT

PSMR - PETROLEUM SUPPLY MONTHLY

STKS - PSM STATE STOCKS TABIE

WCPR - WEEKLY COAL PRODUCTION REPORT

EPMS - U.S. ELECTRIC POWER STATISTICS

NGMR- NATURAL GAS MONTHLY

PLEASE ENTER THE DESIRED REPORT ID... PSMR

4) ENTER YOUR 10 DIGIT PHONE NUMBER.

\$WP1081 LOGON IN PROGRESS AT 13:23:22 ON MAY 9, 1989

PLEASE ENTER YOUR PHONE NUMBER...

5) YOU WILL THEN SEE A BANNER WHICH SHOWS THE REPORT YOU HAVE SELECTED AND PAUSES TO ALLOW AMPLE TIME TO GET READY'TO RECEIVE OUTPUT.

YOU HAVE SELECTED MONTHLY STATISTICS FROM PEIROLEUM SUPPLY MONTHLY (PSM) SYSTEM. THIS SYSTEM WILL DISHLAY THE MOST RECENT PSM DATA FOR TABLES 4, 16, 25, 41, AND 42. PLFASE TURNON YOUR PRINTER NOY IFYOUWISHTOOBTAINHARD COPY OUTPUT.

(PRINTING WIIL BEGIN IN 20 SI:CONDS)

Note: Users who experience problems when first ancumbing lo begon should check their lerminal switch sellings for the following:

- 7 Dalía Bits

- 1 Siop Bir

- Even Parity 


\section{Preface}

The Natural Gas Momhly (NGM) is prepared in the Data Operations Branch of the Reserves and Natural Gas Division, Office of Oil and Gas, Energy Information Administration (EIA), U.S. Department of Energy (DOE).

General questions and comments regarding the $N G M$ may be referred to Kendrick E. Brown, Jr. (202) 586-6077. James M. Todaro, (202) 586-6305, or Eva M. Fleming, (202) 586-6113. Specific technical questions may be referred to the appropriate persons listed in Appendix E.

The NGM highlights activities, events, and analyses of interest to public and private sector organizations associated with the natural gas industry. Volume and price' sta are presented each month for natural gas production, distribution, consumption, and interstate pipeline activities. Producer-related activities and underground storage data are also reported. From time to time, the $N G M$ features articles designed to assist readers in using and interpreting natural gas information.

The data in this publication are collected on surveys conducted by the ElA to fulfill its responsibilities for gathering and reporting energy data. Some of the data are collected under the authority of the Federal Energy Regulatory Commission (FERC), an independent commission within the DOE, which has jurisdiction primarily in the regulation of electric utilities and the interstate natural gas industry.

Explanatory Notes supplement the information found in tables of the report. A description of the data collection surveys that support the $N G M$ is provided in the Data Sources section. A glossary of the terms used in this report is also provided to assist readers in understanding the data presented in this publication.

All natural gas volumes are reported at a pressure base of 14.73 pounds per square inch absolute (psia) and at 60 degrees Fahrenheit. 


\section{Common Acronyms Used in the Natural Gas Monthly}

\begin{tabular}{|c|c|}
\hline$\triangle(i . A$ & American Cias Amociation \\
\hline$|3 h|$ & Barrels \\
\hline$B 1.5$ & $\begin{array}{l}\text { Burcatt of } 1 \text { abor Statistices l L.S. } \\
\text { Department of } 1 \text { abor }\end{array}$ \\
\hline$B C f^{\circ}$ & Billion Cubic Feel \\
\hline BO( ) 1 & $\begin{array}{l}\text { Burcau of Nimes, Ls. Depart- } \\
\text { ment of the Interior }\end{array}$ \\
\hline BH & Britioh Thermal Unit \\
\hline 1$)(3)$ & L.s. Department of Energey \\
\hline$D() I$ & U.S. Departmeist of the Interion \\
\hline I:IA & $\begin{array}{l}\text { Energy Information Administra- } \\
\text { tom. (I.S. Department of Energy }\end{array}$ \\
\hline IIERC & $\begin{array}{l}\text { Federal Energey Regulatory } \\
\text { Comminson }\end{array}$ \\
\hline
\end{tabular}

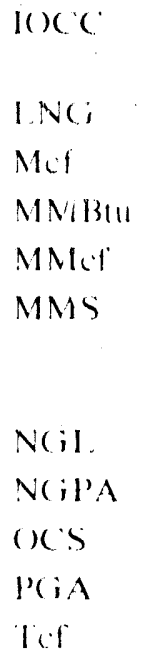

Interstate ()il (ompalat (ommissicill

1.ipuefied Natural (ias

Thousand Cuhic leed

Millon British Themal lnits

Million Cubic lecet

Enited States Mincrals Manage-

ment Sorvices L.S. Department

(1) the Interion

Nallual Cias liguids

Nallural Cias Policy Act of 1978

(oner Continental shelf

Purchased Cias Adjustment

Prillion Cubic Ped 


\section{Contents}

() Nervicil

Appendices

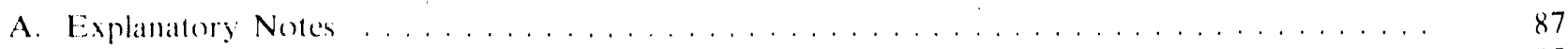

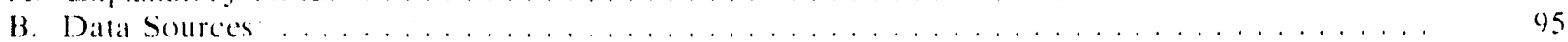

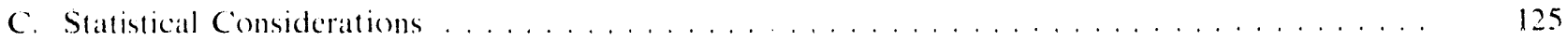

D. Natural Gas Reports and Feature Articles . . . . . . . . . . . . . . . . . . . . . . . .

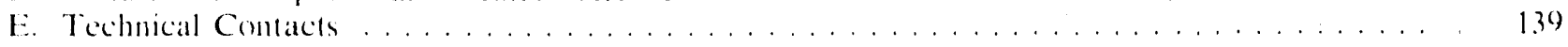

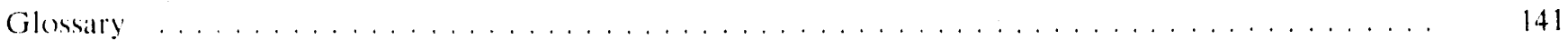

\section{Tables}

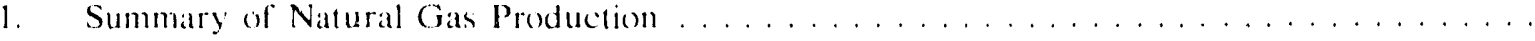

Supply and Disposition of Dry Natural Gas . . . . . . . . . . . . . . . . . .

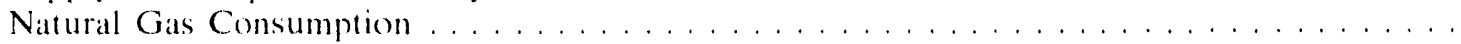

Selected National Average Natural Gas Prices . . . . . . . . . . . . . . . . . .

Projected Volumes and Prices of Wellhead Purchases by NGPA Category . . . . . . . . . .

Summary of Natural Gas Imports and Exports . . . . . . . . . . . . . . . . . .

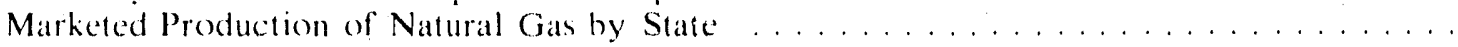

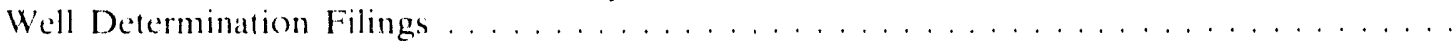

Well Determination Filings by State, September $1900 \ldots \ldots \ldots \ldots \ldots \ldots \ldots$

Well Determination ilings by Category, September $1990 \ldots \ldots \ldots \ldots \ldots$

Natural Gas Ceiling Prices by Category of Gas, Type of Sale, or Contract . . . . . . . . . . .

Revenues, Expenses, and Income of Major Interstate Natural Gas Pipeline Companies . . . .

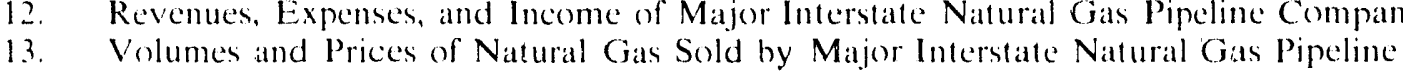

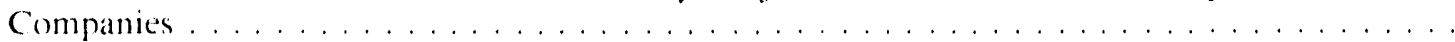

14. Volumes and Prices of Natural Gas Sold by Major Interstate Natural Gas Pipeline Compa-

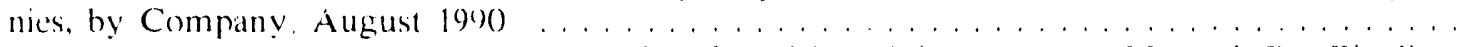

15. Natural and Other Gases Produced and Purchased by Major Interstate Natural Gas Pipeline

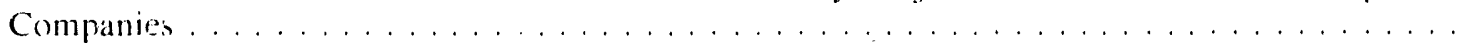
Natual and Other Gases Produced and Purchased by Major Interstate Natural Gas Pipeline

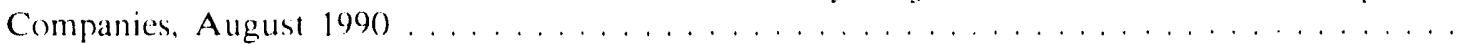
Underground Natural Gas Storage - All operators . . . . . . . . . . . . . . . . .

18. Underground Natural Gas Storage - Interstate Operators of Storage Fields . . . . . . . . . . Underground Natural Gas Storage - Intrastate Operators and Independent Producers . . . . Activities of Multi-State Undergroand Natural Gas Storage Operators, September 190() ... Activities of Single-State Underground Natural Gas Stotage Operators, by State, September 1990

Natural Gas Deliveries to Residential Comsumers by state . . . . . . . . . . . . . . .

Natural Gas Deliveries of Commercial Consumers by Statc . . . . . . . . . . . . .

Natural Cias Deliveries to Industrial Comsumers by State . . . . . . . . . . . . . . . .

Natural Gas Deliveries 10 Electric Utility Comsumers by State . . . . . . . . . . . . . .

Natural Gas Deliveries to All Comsumers by Statc . . . . . . . . . . . . . . .

Average City Ciate Price by State.

Average Price of Natural Gas Delidered to Residential Comsmomers hy Stalle

Average Prece of Natural cias Sold lo Commercial Comstumers by State 
30. Average Price of Natural Gas Sold to Industrial Consumers by State . . . . . . . . . . . . . 64

31. Average Price of Natural Gas Delivered to Electric Utility Consumers by State . . . . . . . 67

32. Average Price of Natural Gas Delivered to All Consumers by State . . . . . . . . . . . . . . . 70

33. Percentage of Total Deliveries Represented by Onsystem Sales . . . . . . . . . . . . . . . . 73

C1. Standard Error for Natural Gas Deliveries and Price to Consumers by State, August 1990 .

\section{Illustrations}

Production and Consumption of Natural Gas in the United States . . . . . . . . . . . . . . . . . . . . . . . . . . .

Page

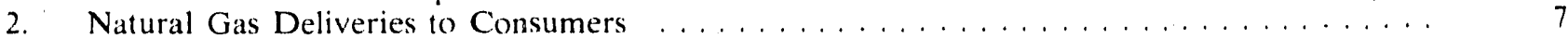

3. Average Price of Natural Gas Delivered to Consumers . . . . . . . . . . . . . . . . . . .

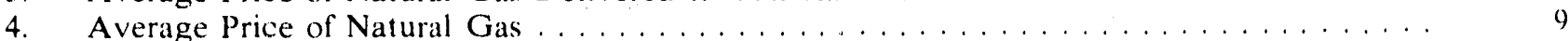

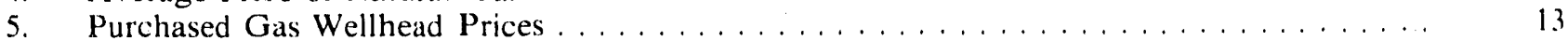

6. Underground Natural Gas Storage in the United States . . . . . . . . . . . . . . . . . . . . . . . . . .

7. Percentage of Total Deliveries Represented by Onsystem Saies . . . . . . . . . . . . . . . . . . 79 


\section{Overview}

\section{Supply and Disposition}

The natural gas market remained relatively stable during September, a month dominated by the conflict in the Mideast and the attendant surge in oil prices. As in August, when the comflict began, September activity in the natural gas industry largely followed seasomal patterns.

The Mideast conflict has resulted in litte fuel switching. Most fuel switching had already occurred because the price of natural gas was lower even before the dramatic rise in oil prices. As reported in last month's $N G, 1$, for the year to date, warmer than normal weather during the first quarter and a sluggish economy kept demand and prices lower than might otherwise have been expected. The effects of these lower prices on production and demand are discussed below.

ElA estimates that marketed production (gross withdrawals less gas used for repressuring, quantities vented and Mared, and nonhydrocarbon gases removed) totaled 1,403 billion cubic feet, down 4.5 percent from August's 1,469 billion cubic feet (a typical decline for this time of year). A year-to-date comparison shows that marketed production in 1990) is so far running just 1 percent higher than in 1989,2 pereent higher than in 1988.

The lotal gas supply available for disposition in September was estimated at 1,504 billion cubic feet (almost 4 percent less than in August 1990) but virtually the same as in September 1989). Shown in Table 2, this tolal includes 36 billion cubic feet withdrawn from storage, 11 billion cubic feet of supplemental fuel supplies, and 120 billion cubic feet of imported gas. Imports, predominantly from Canada, accounted for slightly more than 7 percent for both the month and the year as a whole. Imports have been increasing since 1987 because they have been priced below domestic onsystem sales by 8 to 13 cents per thousand cubic feet.

On the disposition side, total consumption decreased 10 1,2.35 billion cubic feet, 284 hillion rubie feet were injected into underground storage reservoirs, and 8 billion cubic feet were exported. This leaves 23 billion cubic fect unaceounted for.

\section{Consumption}

Natural gas consumption increased from July to August, by 2.2 percent. Total consumption is up slightly for the year compared with the same period of 1989. Energy demand closely tracks economic growth and as would be expected with the nation's economy slowing, demand for all fossil fuels is down during this period.

Only in the industrial sector has consumption shown any significant growth: totaling 4,676 billion cubic feet for the year to date. Consumption is 4.6 percent higher than for the same period of 1989; from July to August, the increase amounted to almosi 4 percent. This rate of growth, while stronger than in the other consuming sectors, is somewhat weaker than the 7-percent annual growth witnessed from 1986 to 1989. The slowdown in the economy is taking its toll on this sector as well as the others. At the same time, the relatively low price of natural gas probably kept demand from declining further than would be expected in a low economy.

The electric utility sector typically consumes more natural gas during the summer months, when spaceheating demands are weakest. Following this seasonal pattern, consumption increased by over 6 percent from July to August (compared with 10 percent from June to July). But consumption is still off by almost 2 percent for the year, as compared with the same period in 1989. Total electric generation is up less than 2 percent for the year, with only nuclear power and hydroelectric gencration showing any substantial growth.

In both the residential and commercial sectors, demand for natural gat: is predominantly driven by space heating requirements. With the warmer than normal first quarter, year-to-date residential consumption is almost 6 percent lower than during the same period of 1989 $(3,034$ billion cubic feet compared with 3,214 billion cubic feet); and commercial consumption is virtually the same $(1,821$ billion cubic feet compared with 1,831 billion cubic feet).

Following typical seasonal patterns, consumption of natural gas has declined maskedly since the winter months in both these sectors: August demand dropped to 124 billion cubic feet in the residential sector, down from 129 billion cubic feet in July; and to 119 billion 
cubic feet in the commercial sector, down from 128 billion cubic lece in luly,

\section{Prices}

As moted ahove, Irag's invasion of Kuwait and the dramatic increase in oil prices has nol caused any substantial increase in natural gas prices. Natural gats prices were already relatively low in 1000), a function of the spot market and gas 10 gats competition. Obviously, these low prices have kept natural gas comnetitive with other fuels throughout the year. It will te some time, if at all, before the surge in oil prices has any effect on natural gas prices.

\section{As shewe in Table 4.}

- According to EIÁs estimates, in July (the last menth for which data are available) wellhead prices averaged $\$ 1.5()$ per thousand cubic feet. down from $\$ 1.53$ per thousand cubic feet in both May and June; wellhead prices averaged $\$ 1.65$ per theosand cubic feet in July 1989, about 9 percent more

- In August 1990, major interstate pipeline companies paid an average of $\$ 1.86$ per thousand cubic fiel for gas purcl.ased from domestic producers, down from $\$ 2.0()$ per thousand cubic feet in July. In August these pipeline companies paid, on avcrage, \$1.93 for imported gas, almost all of it Canadian. Although higher than domestic prices (onsystem sales) in August, imports have heen 8 cents cheaper than domestic for the year as a whole.

- Distributors paid an average $\$ 2.91$ per theousand cubic feet at the city galle in August, approximalcly 4 percent less than the $\$ 3.3 .0 .3$ paid in July 1990 or the \$3.04 paid in August 1989.

- Industrial consumers paid an average of $\$ 2.52$ per thousand cubic feet. This is a 2 percent increase form July's \$2.47, which was less than in any momth in recent history. Fin the geall lo dater industrial consumers are patying 2 pereent less than they did for year-to-date loge but exaldy the same as for year-fo-dalce 1988.

- Commercial comsumers paid $\$ 4.55$ per lhousand cubic feet in August, 2 pereent more than the $\$ 4.46$ they paid in July. 2 pereent less than the $\$ 4.65$ paid in August 1989. For the year as a whole, prices are running ? perecont higher than ycar-to-date 1989.

- Residential consumers paid \$7.04 per thousand cubic feet, less than 1 percent more than the $\$ 6.99$ paid in July, and almost .5 percent less than the $\$ 7.07$ paid in August 1989. Residential prices are traditionally higher in the summer months because demand is lower while fixed costs remain. For the year to date, residential consumer prices are running almost 2 pereent above year-to-date 1989 prices, and almost 7 percent above year-todate 1988 prices.

- Electric utilities paid an average of \$2.22 per thousand cubic fect in July. This is an increase of 3 percent from the $\$ 2.16$ atilities paid in Junc, a decline of 7.5 percent from July 1989. So far in 199(), electric utility prices are rumning 2 percent below 1989, 3 pereent above 1988 prices.

\section{Underground Storage}

\begin{abstract}
A majority of the available natural gas (gas mot needed to meet eonsumer demand) was placed in underground storage facilities to prepare for winter heating requirements. As shown in Table 17, in September, 284 billion cubic feet were injected inter storage and 37 billion cubic feet withdrawn. This brings the total gas in storage 107,119 billion cubic feet, a new record. The warm weather allows storage facilities to place so much gas in storage. This amount is almost 2 pereent greater than the storage level of $\left.6,{ }^{(6)}\right)$ reported in Sepiember 1989.
\end{abstract}


Table 1. Summary of Natural Gas Production (Billion Cubic Feet)

\begin{tabular}{|c|c|c|c|c|c|c|c|}
\hline $\begin{array}{l}\text { Year } \\
\text { and } \\
\text { Month }\end{array}$ & $\begin{array}{c}\text { Gross } \\
\text { Withdrawals }\end{array}$ & Repressuring & $\begin{array}{c}\text { Nonhydrocarbon } \\
\text { Gases } \\
\text { Hemoved }\end{array}$ & $\begin{array}{l}\text { Vonted } \\
\text { and } \\
\text { Flared }\end{array}$ & $\begin{array}{l}\text { Markeled } \\
\text { Production } \\
\text { (Wel) }\end{array}$ & $\begin{array}{c}\text { Extraction } \\
\text { Loss }\end{array}$ & $\begin{array}{c}\text { Tolal Dry } \\
\text { Gas } \\
\text { Production }\end{array}$ \\
\hline 1984 Total & 20,192 & 1,630 & 224 & 108 & 18,230 & 838 & 17,392 \\
\hline 1985 Total & $19,53 A$ & 1,915 & 326 & 95 & 17,198 & 816 & 16,382 \\
\hline 1986 Total & 19,063 & 1,838 & 337 & 98 & 16,791 & 800 & 15,991 \\
\hline 1987 Total & 20,056 & 2.208 & 376 & 124 & 17,349 & 812 & 16,536 \\
\hline \multicolumn{8}{|l|}{1988} \\
\hline January ................. & 1,925 & 216 & 40 & 12 & 1,657 & 76 & 1,581 \\
\hline February & 1.752 & 196 & 36 & 12 & 1,508 & 69 & 1,439 \\
\hline 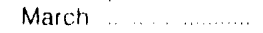 & 1,826 & $20^{\prime}$ & 40 & 12 & 1,573 & 72 & 1,501 \\
\hline Apı & 1.684 & 19.1 & 39 & 12 & 1,440 & 66 & 1,374 \\
\hline 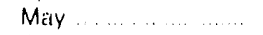 & 1.724 & 204 & 33 & 12 & 1,475 & 68 & 1,407 \\
\hline June $\ldots \ldots \ldots \ldots$ & 1.655 & 202 & 39 & 12 & 1,402 & 64 & 1,338 \\
\hline July $\ldots \ldots \ldots \ldots$ & 1.674 & 204 & 37 & 13 & 1,420 & 65 & 1,355 \\
\hline 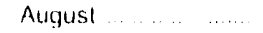 & 1,691 & 203 & 36 & 12 & 1,440 & 66 & 1,374 \\
\hline September $\ldots$ & 1,609 & 200 & 38 & 12 & 1,359 & 62 & 1,297 \\
\hline 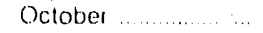 & 1,747 & 217 & 42 & 12 & 1,476 & 67 & 1,409 \\
\hline November ............ & 1,772 & 217 & 38 & 12 & 1,505 & 69 & 1,436 \\
\hline Docember $\ldots$..... & 1,864 & 225 & 42 & 11 & 1,586 & 73 & 1,513 \\
\hline Total $\ldots \ldots \ldots$ & 20.922 & 2.478 & 460 & 143 & 17,841 & 817 & 17,026 \\
\hline \multicolumn{8}{|l|}{1989} \\
\hline January & 1,859 & 217 & 34 & 11 & 1,597 & 70 & 1,527 \\
\hline February & 1,709 & 191 & 29 & 11 & 1,476 & 64 & 1,412 \\
\hline 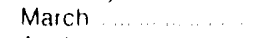 & 1.804 & 195 & 31 & 13 & 1,564 & 68 & 1,496 \\
\hline April $\ldots \ldots \ldots$ & 1.734 & 201 & 29 & 12 & 1,491 & 65 & 1,426 \\
\hline 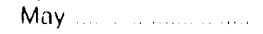 & 1.766 & 212 & 31 & 12 & 1,511 & 66 & 1,445 \\
\hline June $\ldots . . . . . . . . . . . .$. & 1.677 & 190 & 28 & 12 & 1,449 & 63 & 1,386 \\
\hline July & 1.710 & 197 & 30 & 12 & 1,474 & 64 & 1,410 \\
\hline August ...................... & 1,701 & 205 & 28 & 12 & 1,460 & 63 & 1,397 \\
\hline September ......... & 1.637 & 205 & 28 & 12 & 1,393 & 60 & 1,333 \\
\hline October & 1.718 & 209 & 29 & 12 & 1,469 & 64 & 1,405 \\
\hline November ............ & 1.782 & 213 & 31 & 12 & 1.525 & 66 & 1,459 \\
\hline December ....... & 1,901 & 217 & 33 & 12 & 1,635 & 72 & 1,563 \\
\hline$\ldots \ldots \ldots \ldots$ & 20,998 & 2.451 & 362 & 141 & 19,044 & 785 & 17,260 \\
\hline \multicolumn{8}{|l|}{1990} \\
\hline January ............... & 1.928 & 205 & 32 & 15 & 1,676 & 78 & 1,598 \\
\hline 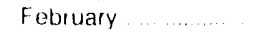 & 1,708 & 180 & 27 & 9 & 1,492 & 70 & 1,422 \\
\hline March & 1.815 & 207 & 30 & 10 & 1,568 & 73 & 1,495 \\
\hline April & 1,737 & 201 & 29 & 10 & 1.497 & 70 & 1.427 \\
\hline May $\ldots \ldots$ & 1.772 & 203 & 35 & 11 & 1,523 & 71 & 1,452 \\
\hline June & 1,698 & 191 & 29 & 10 & 1,468 & 69 & 1,399 \\
\hline July $\ldots$ & A 1.701 & ค 194 & A 30 & ค 10 & R 1,467 & 69 & ค 1,398 \\
\hline Auçusi & E 1.708 & E 195 & E 33 & E 11 & E 1,469 & E 69 & E 1,400 \\
\hline September & E. 1,627 & E 185 & 29 & E 10 & E 1,403 & E 66 & E. 1,337 \\
\hline 1990 YTD & 15,694 & 1.761 & 274 & 96 & 13,563 & 635 & 12,928 \\
\hline 1989 Y Y D & 15.597 & 1,813 & 268 & 107 & 13,416 & 583 & 12,832 \\
\hline 1988 YTD & 15.540 & 1.819 & 338 & 109 & 13,275 & 608 & 12,666 \\
\hline
\end{tabular}

\footnotetext{
a See Appendix A. Explanatory Note 1 lor a discussion of data on Nonhydrocarbon Gases Removed - Equal to marketed production (wet) minus extraction loss.

E Estimaterd Data

H Fieviseo Data

Notes and Sources. Soe the last page of this section
} 
Table 2. Supply and Disposition of Dry Natural Gas (Billion Cubic Feet)

\begin{tabular}{|c|c|c|c|c|c|c|c|c|c|}
\hline & & & pply & & & & & Disposilion & \\
\hline Year & & 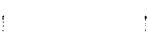 & , & & Tolal & & & & 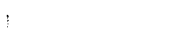 \\
\hline $\begin{array}{c}\text { and } \\
\text { Month }\end{array}$ & $\begin{array}{l}\text { Total Dry } \\
\text { Gas } \\
\text { Production }\end{array}$ & $\begin{array}{c}\text { Withdrawals } \\
\text { from } \\
\text { Storagea }\end{array}$ & $\begin{array}{l}\text { Supplemental } \\
\text { Gaseous } \\
\text { Fuels }\end{array}$ & Imports & $\begin{array}{c}\text { Supply/ } \\
\text { Disposition }\end{array}$ & $\begin{array}{c}\text { Additions } \\
10 \\
\text { Storagea }\end{array}$ & Exports & Consumption ${ }^{c}$ & $\begin{array}{l}\text { Unaccounted } \\
\text { Ford }\end{array}$ \\
\hline 1984 Tolal & 17,392 & 2,098 & 110 & 843 & 20,443 & 2,295 & 55 & 17,951 & 143 \\
\hline 1985 Total & 16.382 & 2,397 & 126 & 950 & 19,855 & 2,163 & 55 & 17,281 & 356 \\
\hline 1986 Total $\ldots . . . . . . . . .$. & 15,991 & 1,837 & 113 & 750 & 18,692 & 1,984 & 61 & 16,221 & 427 \\
\hline 1987 Total & 16,536 & 1.905 & 101 & 993 & 19,534 & 1,911 & 54 & 17.211 & 359 \\
\hline 1988 & & & & & & & & & \\
\hline January & 1,581 & 586 & 12 & 139 & 2,318 & 47 & 5 & 2,187 & 79 \\
\hline February & 1.439 & 462 & 11 & 117 & 2,029 & 50 & 5 & 2,038 & .64 \\
\hline March & 1,501 & 259 & 10 & 113 & 1,883 & 99 & 6 & 1,867 & .89 \\
\hline 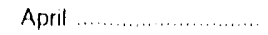 & 1,374 & 92 & 8 & 96 & $1,5 \% 0$ & 165 & 6 & 1.464 & -65 \\
\hline 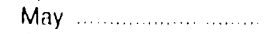 & 1,407 & 46 & 7 & 94 & 1,554 & 288 & 4 & 1.302 & .40 \\
\hline 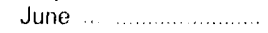 & 1,338 & 36 & 7 & 93 & 1,474 & 280 & 8 & 1,170 & 16 \\
\hline 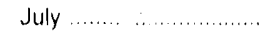 & 1,355 & $4 ?$ & 7 & 100 & 1,504 & 300 & 5 & 1.177 & 22 \\
\hline 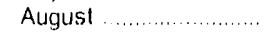 & $1,37 i$ & 52 & 7 & 94 & 1.527 & 288 & 6 & 1,222 & 11 \\
\hline September .............. & 1,297 & 46 & 6 & 95 & 1,444 & 314 & 7 & 1,099 & 24 \\
\hline 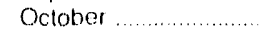 & 1,409 & 92 & $B$ & 106 & 1,615 & 202 & 6 & 1,232 & 175 \\
\hline Novernber ..................... & 1.436 & 159 & 9 & 12.1 & 1.725 & 117 & 7 & 1.453 & 148 \\
\hline December .................. & 1,513 & 397 & 11 & 127 & 2,048 & 62 & 9 & 1,820 & 157 \\
\hline 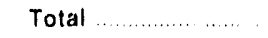 & 17.026 & 2.270 & 101 & 1,294 & 20,691 & 2,211 & 74 & 18,030 & 376 \\
\hline 1989 & & & & & $\cdot$ & & & & \\
\hline January .................... & 1,527 & 426 & 11 & 119 & 2.0833 & 53 & 7 & 2,023 & 0 \\
\hline Fotruary ....................... & 1,412 & 61.4 & 10 & 110 & 2.146 & 32 & 7 & 2,008 & 99 \\
\hline 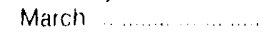 & 1.196 & 369 & 10 & 113 & 1,988 & 106 & 11 & 1.945 & -74 \\
\hline 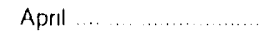 & 1.426 & 138 & 8 & 110 & $1,68 ?$ & 184 & 11 & 1,580 & .93 \\
\hline 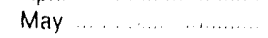 & 1.445 & 44 & 8 & 108 & 1,605 & 326 & 8 & 1,348 & -77 \\
\hline June ..................... & 1,386 & 20 & 7 & 104 & 1,517 & 381 & 9 & 1,200 & -73 \\
\hline July ........................ & 1.410 & 29 & 8 & 101 & 1.548 & 377 & 9 & 1,220 & $-5 ! 3$ \\
\hline August & 1,397 & 29 & 8 & 108 & 1,542 & 362 & 9 & 1,216 & .45 \\
\hline Suptember & 1,333 & 39 & 7 & 117 & 1,496 & 325 & 9 & 1,181 & -19 \\
\hline October . . . & 1,405 & 96 & 9 & 123 & 1,633 & 225 & 10 & 1,337 & 61 \\
\hline Novemtser $\ldots \ldots \ldots$ & 1.459 & 227 & 9 & 123 & 1,818 & 105 & 8 & 1.567 & 138 \\
\hline Decernter $\quad . . . . . . . .$. & 1,563 & 82.1 & 12 & 145 & 2,541 & 52 & 8 & 2.156 & 325 \\
\hline Total & 17,260 & 2,852 & 107 & 1.382 & 21,599 & 2,529 & 107 & $18, i 80$ & 182 \\
\hline 1990 & & & & & & & & & \\
\hline January & 1.598 & 339 & 16 & 149 & 2.102 & 91 & 8 & R 2.107 & A .104 \\
\hline Fobruary & 1,422 & 324 & 14 & 118 & 1,878 & 70 & 8 & R 1.805 & R -5 \\
\hline March & 1.495 & 256 & 14 & 115 & 1,880 & 124 & 10 & A $1.77 t$ & A -30 \\
\hline April. & 1.427 & 140 & 13 & 122 & 1.702 & 183 & 8 & ค 1,584 & в ...73 \\
\hline$\ldots \ldots$ & 1.452. & 45 & 11 & 108 & 1.616 & 2899 & 8 & A 1,397 & A $-7 B$ \\
\hline$. \quad .$. & 1,399 & 42 & 11 & 114 & 1.566 & 327 & 9 & H 1,298 & ค -68 \\
\hline July . . ... & A 1,398 & 27 & 12 & R 119 & A 1.556 & 325 & $\Leftrightarrow$ & H 1,292 & H -69 \\
\hline Augusl ... . & E 1,400 & 37 & 11 & A 118 & A 1,566 & 321 & 8 & A 1,321 & A -84 \\
\hline Seplember & E: 1.397 & 36 & 11 & 120 & 1.504 & 284 & 8 & 1.235 & -23 \\
\hline 1990 YTD & 12.928 & 1.246 & 113 & 1.083 & 15,370 & 2,014 & 75 & 13,915 & -534 \\
\hline 1989 YTD & 12,832 & 1.708 & 77 & 990 & 15.607 & 2.146 & 80 & 13.721 & -340 \\
\hline 1988 YTD & 12,666 & 1.6321 & 75 & 941 & 15,303 & 1.831 & 52 & 13,526 & 106 \\
\hline
\end{tabular}

a Monthly and annual data tor 1984 through 1989 include underground storage and liquefed natural gas storage. Data lor January 1990 forward include underground storage only. See Appendix A. Explanatory Note 7 for discussion of computation procedures.

b "Total" data for 1984 through 1989 do not equal equivalent dala in Table 1 of the 1989 Natural Gas Annual due to the exclusion of intransil receipts and deliveries in the NGM.

- Consists of pipeline fuel use, lease and planl fuet use, and deliveries to consuming sectors as shown in Table 3.

d Fepresents quantities lost and imbalarices in data due to differences among data sources. Soe Appendix 1, Explanatory Nolo 11 for full discussion.

E Estirnated Data

H Pervised Data

Notes and Sources see the last page of this section 
Figure 1. Production and Consumption of Natural Gas in the United States

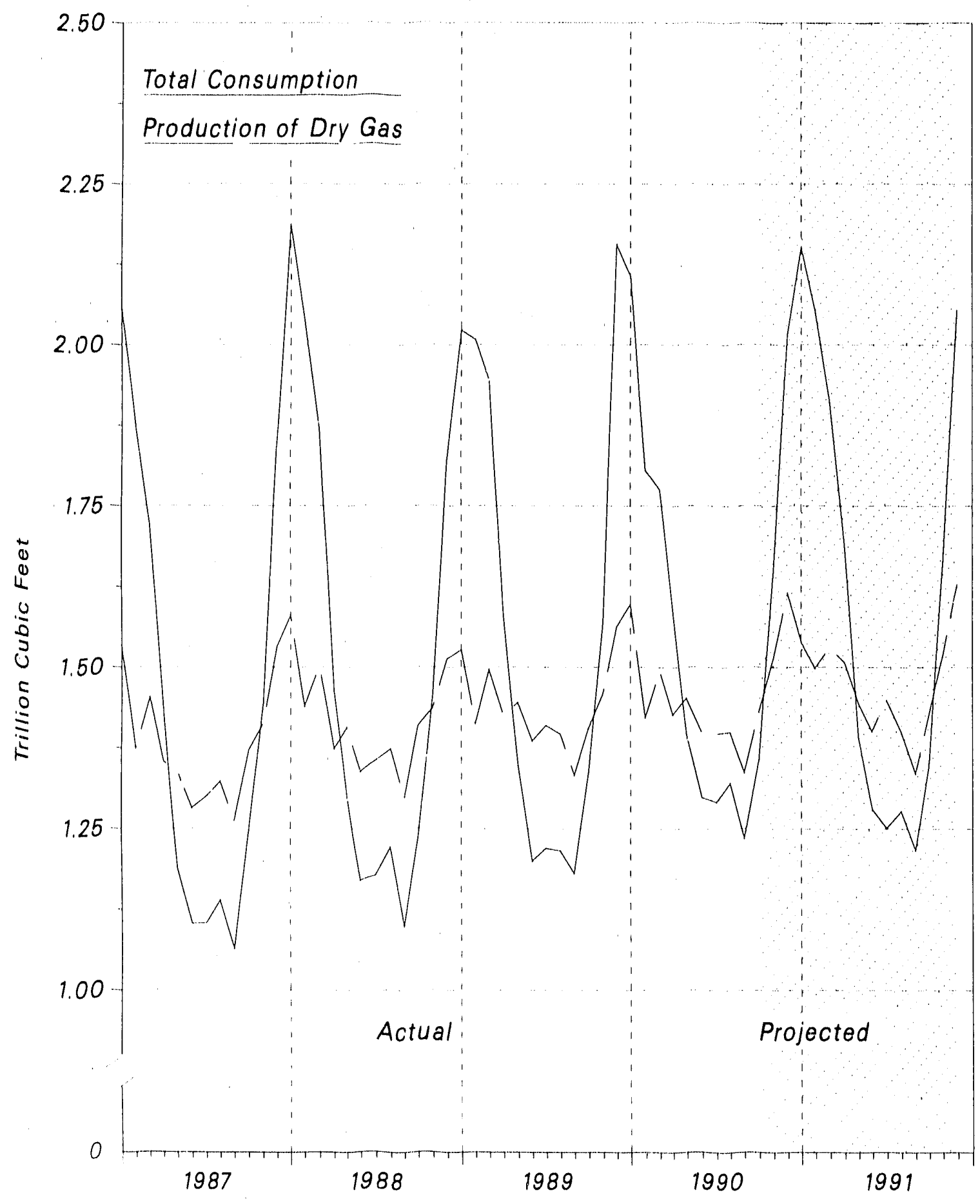

Source: Natural Gas Annual and the Short Term Energy Outlook. 
Table 3. Natural Gas Consumption (Billion Cubic Feet)

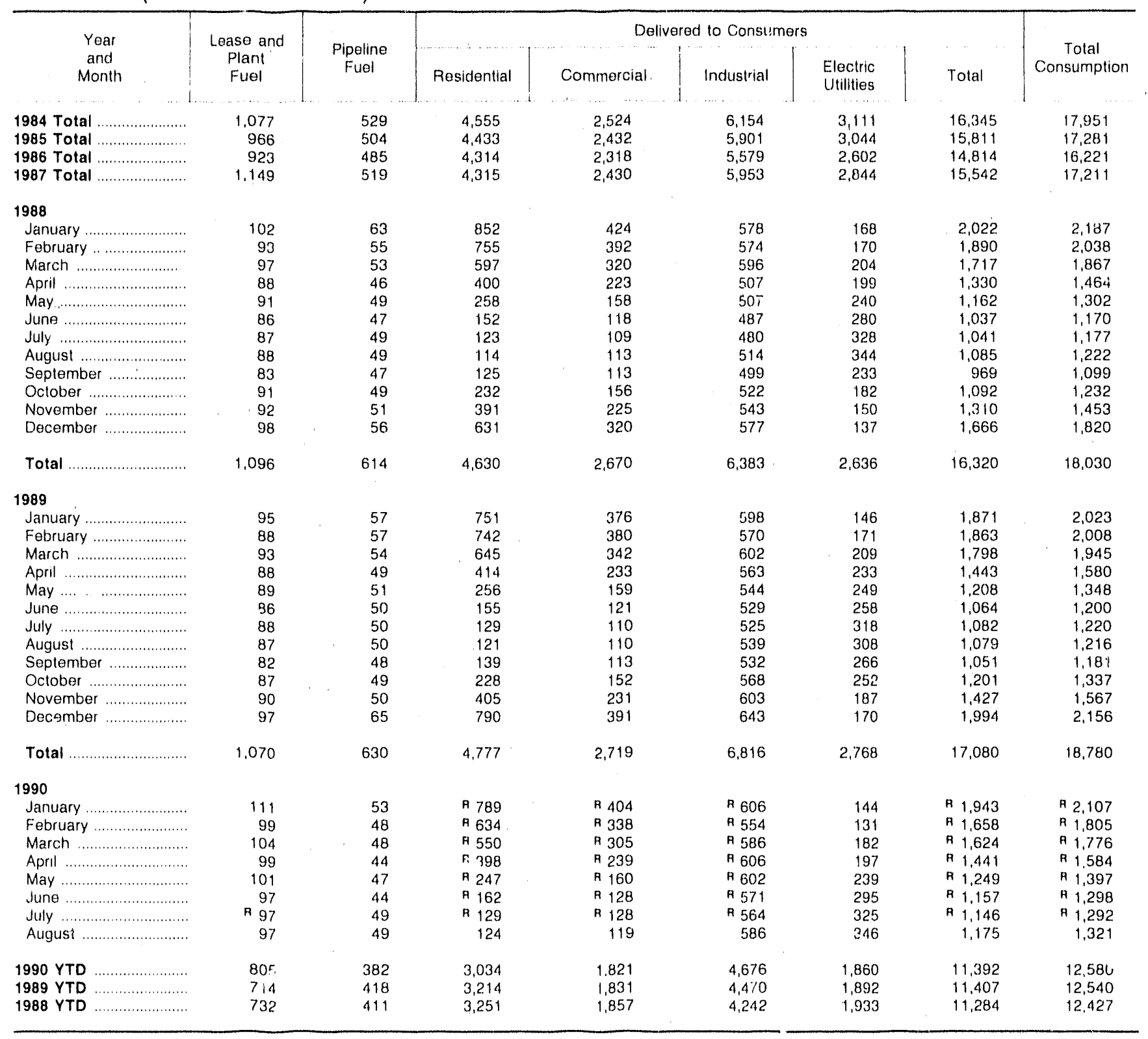

H Revised Data.

Notes and Sources: See the last page of this section. 
Figure 2. Natural Gas Deliveries to Consumers

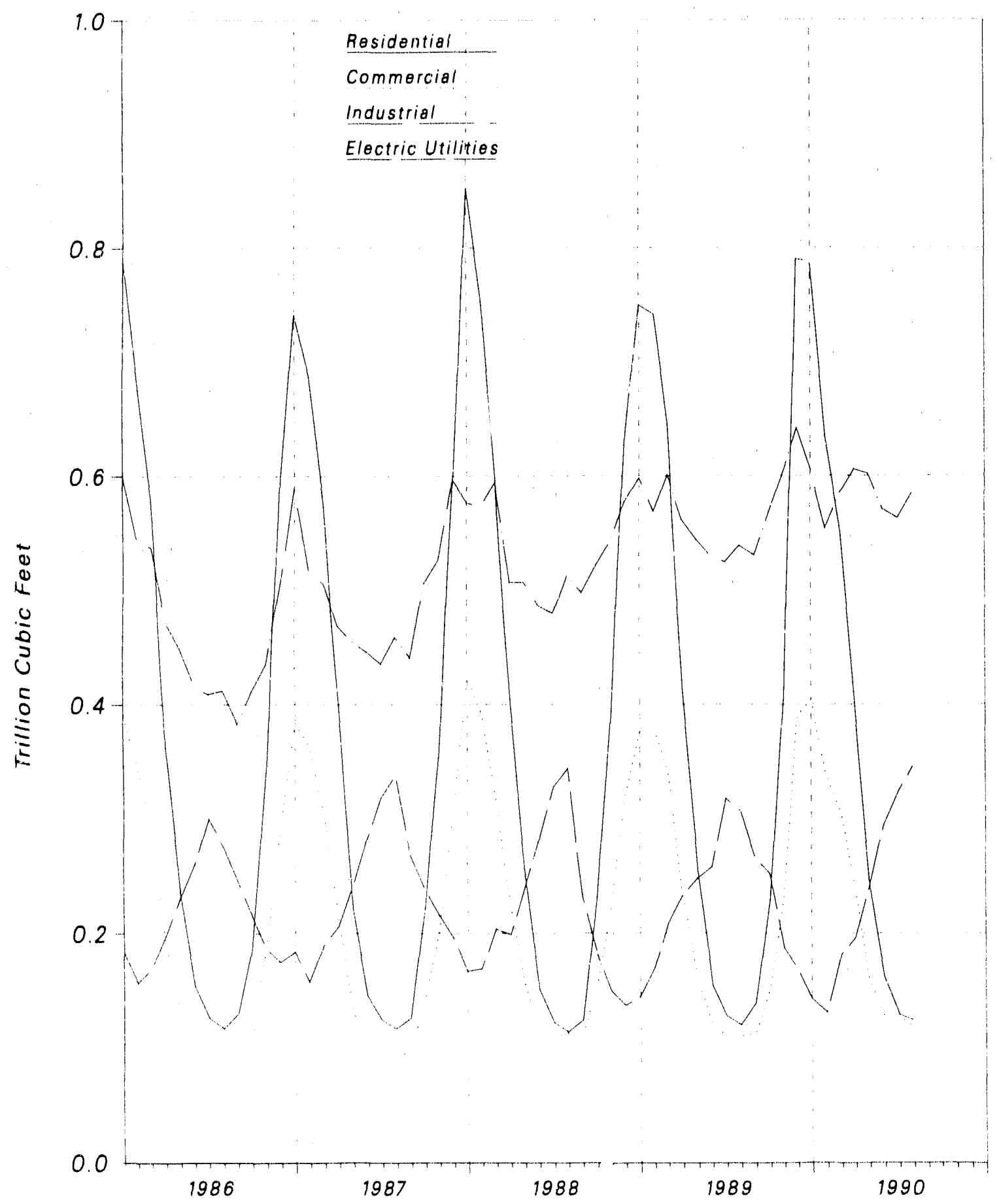

Source: Natural Gas Annual, Form ElA-857, and Form ElA-759. 
Table 4. Selected National Average Natural Gas Prices (Dollars per Thousand Cubic Feet)

\begin{tabular}{|c|c|c|c|c|c|c|c|c|c|}
\hline \multirow{3}{*}{$\begin{array}{c}\text { Year } \\
\text { and } \\
\text { Month }\end{array}$} & \multirow{3}{*}{$\begin{array}{l}\text { Wellhead } \\
\text { Price }^{a}\end{array}$} & \multicolumn{2}{|c|}{$\begin{array}{c}\text { Major Interstate } \\
\text { Pipeline Companies }\end{array}$} & \multirow{3}{*}{$\begin{array}{l}\text { City } \\
\text { Gate }\end{array}$} & \multicolumn{5}{|c|}{ Deliver to Consumers } \\
\hline & & & & & \multirow[b]{2}{*}{ Residential } & \multirow{2}{*}{ Commercialc } & \multirow[b]{2}{*}{ Industria|c } & \multirow{2}{*}{$\begin{array}{l}\text { Electric } \\
\text { Utilities }^{\mathrm{d}}\end{array}$} & \multirow[b]{2}{*}{$\begin{array}{l}\text { Overall } \\
\text { Averagec }^{-}\end{array}$} \\
\hline & & Imports & $\begin{array}{l}\text { Purchased from } \\
\text { Producers }^{b}\end{array}$ & & & & & & \\
\hline 1984 Annual Average $\ldots \ldots \ldots \ldots$ & 2.66 & 4.08 & 2.91 & 3.95 & 6.12 & 5.55 & 4.22 & 3.70 & 4.85 \\
\hline 1985 Annual Average & 2.51 & 3.19 & 2.85 & 3.75 & 6.12 & 5.50 & 3.95 & 3.55 & 4.72 \\
\hline 1986 Annual Average & 1.94 & 2.53 & 2.39 & 3.22 & 5.83 & 5.08 & 3.23 & 2.43 & 4.13 \\
\hline 1987 Annual Average ............. & 1.67 & 2.17 & 2.10 & 2.87 & 5.54 & 4.77 & 2.94 & 2.32 & 4.05 \\
\hline \multicolumn{10}{|l|}{1988} \\
\hline 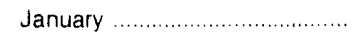 & 1.96 & 1.64 & 2.04 & 2.91 & 5.08 & 4.60 & 3.18 & 2.60 & 4.41 \\
\hline 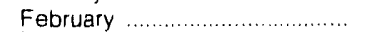 & 1.84 & 2.03 & 2.22 & 2.95 & 5.09 & 4.69 & 3.22 & 2.56 & 4.39 \\
\hline March & 1.70 & 2.09 & 2.03 & 2.87 & 5.18 & .4 .69 & 3.13 & 2.32 & 4.25 \\
\hline April & 1.59 & 2.01 & $2+12$ & 2.79 & 5.35 & 4.71 & 2.97 & 2.20 & 4.10 \\
\hline 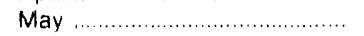 & 1.52 & 2.02 & 2.17 & 2.75 & 5.87 & 4.61 & 2.76 & 2.10 & 3.84 \\
\hline June & $1: 53$ & 1.98 & 205 & 2.87 & 6.50 & 4.53 & 2.67 & 2.16 & 3.54 \\
\hline 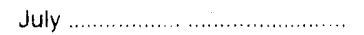 & 1.56 & 2.34 & 1.94 & 2.87 & 6.74 & 4.48 & 2.54 & 2.23 & 3.36 \\
\hline 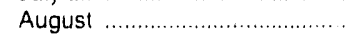 & 1.62 & 1.88 & 2.09 & 2.92 & 6.92 & 4.37 & 2.66 & 2.36 & 3.39 \\
\hline 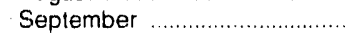 & 1.53 & 2.00 & 2.13 & 3.05 & 6.79 & 4.41 & 2.70 & 2.36 & 3.61 \\
\hline October & 1.68 & 1.94 & 2.31 & 2.92 & 5.95 & 4.53 & 2.80 & 2.40 & 3.95 \\
\hline 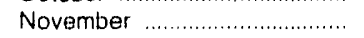 & 1.76 & 1.98 & 2.19 & 2.98 & 5.56 & 4.69 & 3.00 & 2.58 & 4.31 \\
\hline 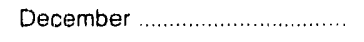 & 1.89 & 2.14 & 2.25 & 3.08 & 5.39 & 4.78 & 3.31 & 2.57 & 4.56 \\
\hline Annual Average $\ldots \ldots \ldots \ldots$ & 1.69 & 2.00 & 2.13 & 2.92 & 5.47 & 4.63 & 2.95 & 2.33 & 4.09 \\
\hline \multicolumn{10}{|l|}{1989} \\
\hline 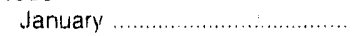 & 1.99 & 1.77 & 2.35 & 3.17 & 5.41 & 4.81 & 3.39 & 2.64 & 4.67 \\
\hline 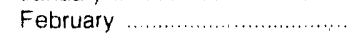 & 1.81 & 2.21 & 2.16 & 3.10 & 5.38 & 4.80 & 3.33 & 2.44 & 4.60 \\
\hline 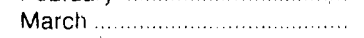 & 1.69 & 1.99 & 2.17 & 2.89 & 5.45 & 4.79 & 3.12 & 2.33 & 4.46 \\
\hline April & 1.56 & 2.01 & 2.22 & 2.83 & 5.54 & 4.77 & 2.91 & 2.31 & 4.18 \\
\hline 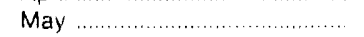 & 1.61 & 2.02 & 2.11 & 2.94 & 5.93 & 4.54 & 2.80 & 2.39 & 3.94 \\
\hline June & 1.65 & 2.04 & 2.04 & 2.98 & 6.58 & 4.57 & 2.69 & 2.40 & 3.72 \\
\hline July & 1.65 & 1.88 & 1.99 & 3.08 & 6.92 & 4.65 & 2.70 & 2.40 & 3.59 \\
\hline 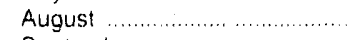 & 1.61 & 2.24 & 2.05 & 3.04 & 7.07 & 4.61 & 2.71 & 2.38 & 3.57 \\
\hline 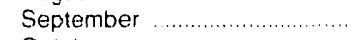 & 155 & 2.02 & 2.07 & 2.99 & 6.80 & 4.67 & 2.67 & 2.33 & 3.67 \\
\hline 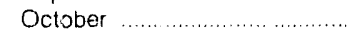 & 1.58 & 2.17 & 2.04 & 2.84 & 6.06 & 4.61 & 2.75 & 2.39 & 3.86 \\
\hline 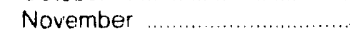 & 1.66 & 2.13 & 2.23 & 2.98 & 5.56 & 4.71 & 2.98 & 2.56 & 4.30 \\
\hline 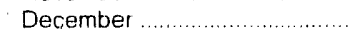 & 1.92 & 2.08 & 2.39 & 3.10 & 5.30 & 4.81 & 3.32 & 2.85 & 4.61 \\
\hline Arınual Average & 1.69 & 2.04 & 2.17 & 3.01 & 5.64 & 4.74 & 2.37 & 2.43 & 4.22 \\
\hline \multicolumn{10}{|l|}{1990} \\
\hline 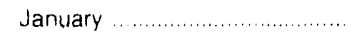 & 2.23 & 2.64 & 2.42 & 3.25 & 5.42 & 4.99 & 3.52 & 3.01 & 4.77 \\
\hline Feuruary $\ldots \ldots \ldots \ldots$ & 1.87 & 2.25 & 2.18 & 3.10 & 5.63 & 5.05 & 3.40 & 2.76 & 4.82 \\
\hline 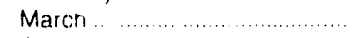 & 1.58 & 1.99 & 1.94 & 2.95 & 5.58 & R 4.93 & 3.08 & 2.37 & 4.50 \\
\hline April & 1.56 & 2.00 & 2.17 & 2.84 & 5.62 & ค 4.82 & 2.84 & 2.29 & 4.23 \\
\hline 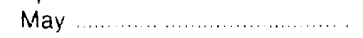 & 1.53 & 2.08 & 1.98 & 2.81 & 5.97 & ค 4.62 & 2.67 & 2.19 & 3.84 \\
\hline June & 153 & 1.91 & 2.18 & 3.00 & 6.55 & 4.59 & 2.55 & 2.16 & 3.53 \\
\hline 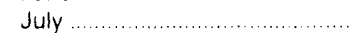 & 1.50 & 1.88 & 2.00 & 3.03 & 6.99 & 4.46 & 2.47 & 2.22 & 3.39 \\
\hline 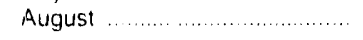 & NA & 1.93 & 1.86 & 2.91 & 7.04 & 4.55 & 2.52 & NA & NA \\
\hline 1990 YTD & NA & 2.01 & 2.09 & 3.01 & 5.75 & 4.85 & 2.93 & 2.35 & 4.29 \\
\hline 1989 YTD . & NA & 202 & 2.14 & 3.01 & 5.65 & 4.75 & 2.99 & 2.40 & 4.28 \\
\hline 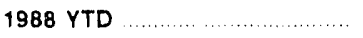 & NA & 2.00 & 2.08 & 2.88 & 5.39 & 4.62 & 2.93 & 2.28 & 4.10 \\
\hline
\end{tabular}

\footnotetext{
a See Appendix A, Explanatory Note 8 for discussion of wellhead price

* See Appendix A. Explanatory Note 9 for discussion of major interstate pipeline company data.

c Ste Table Notes and Sources for explanation of break in series for consurner prices in 1987

d includes all steam electric utility generating plants with a combined capacily of 50 megawatts or greater

A Revised Data

NA Not Available.

Notes and Sources: See the last page of this section.
} 
Figure 3. Average Price of Natural Gas Delivered to Consumers

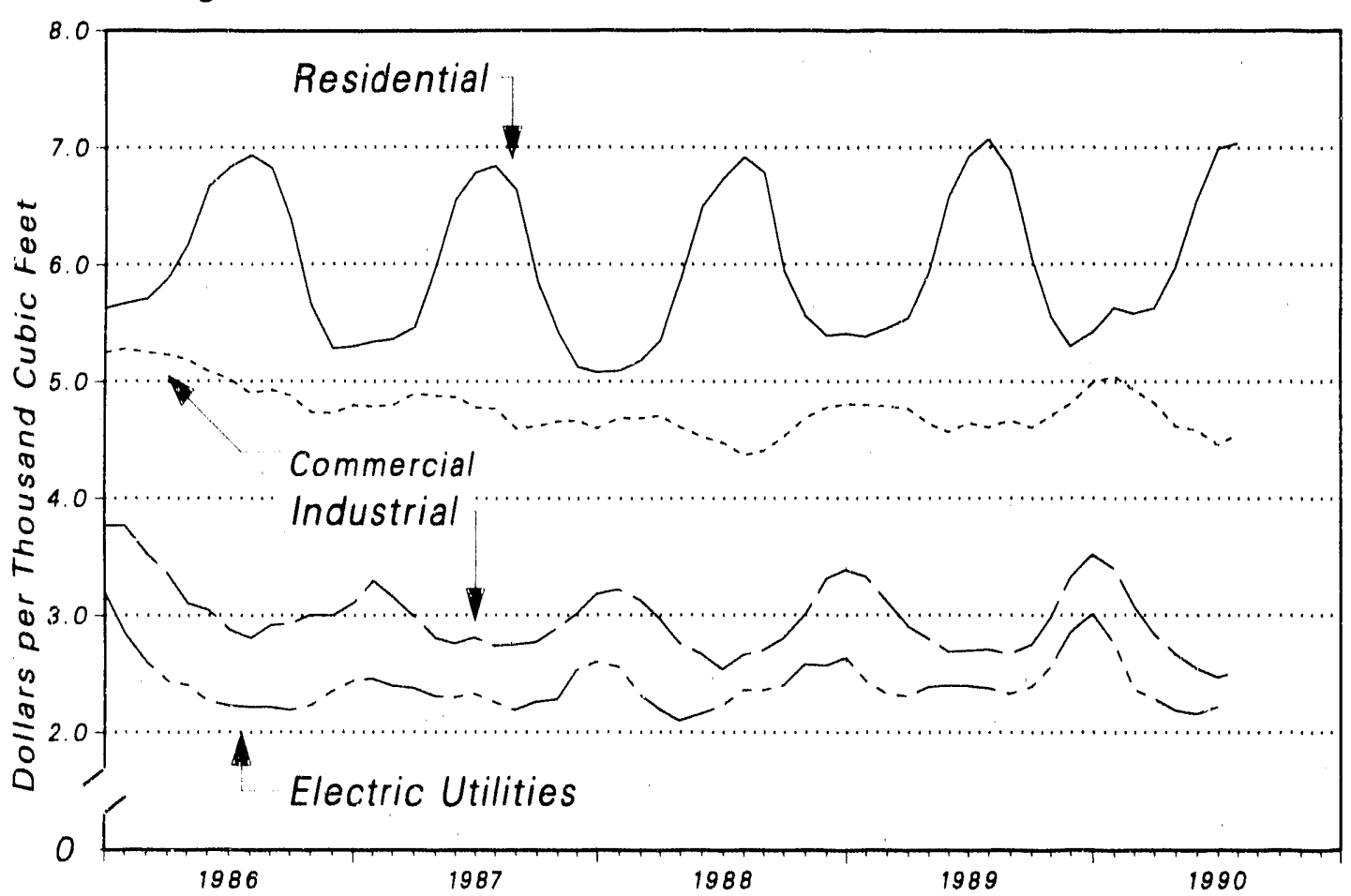

Source: Natural Gas Annual, Form FERC-11, and Form ElA-857.

Figure 4. Average Price of Natural Gas

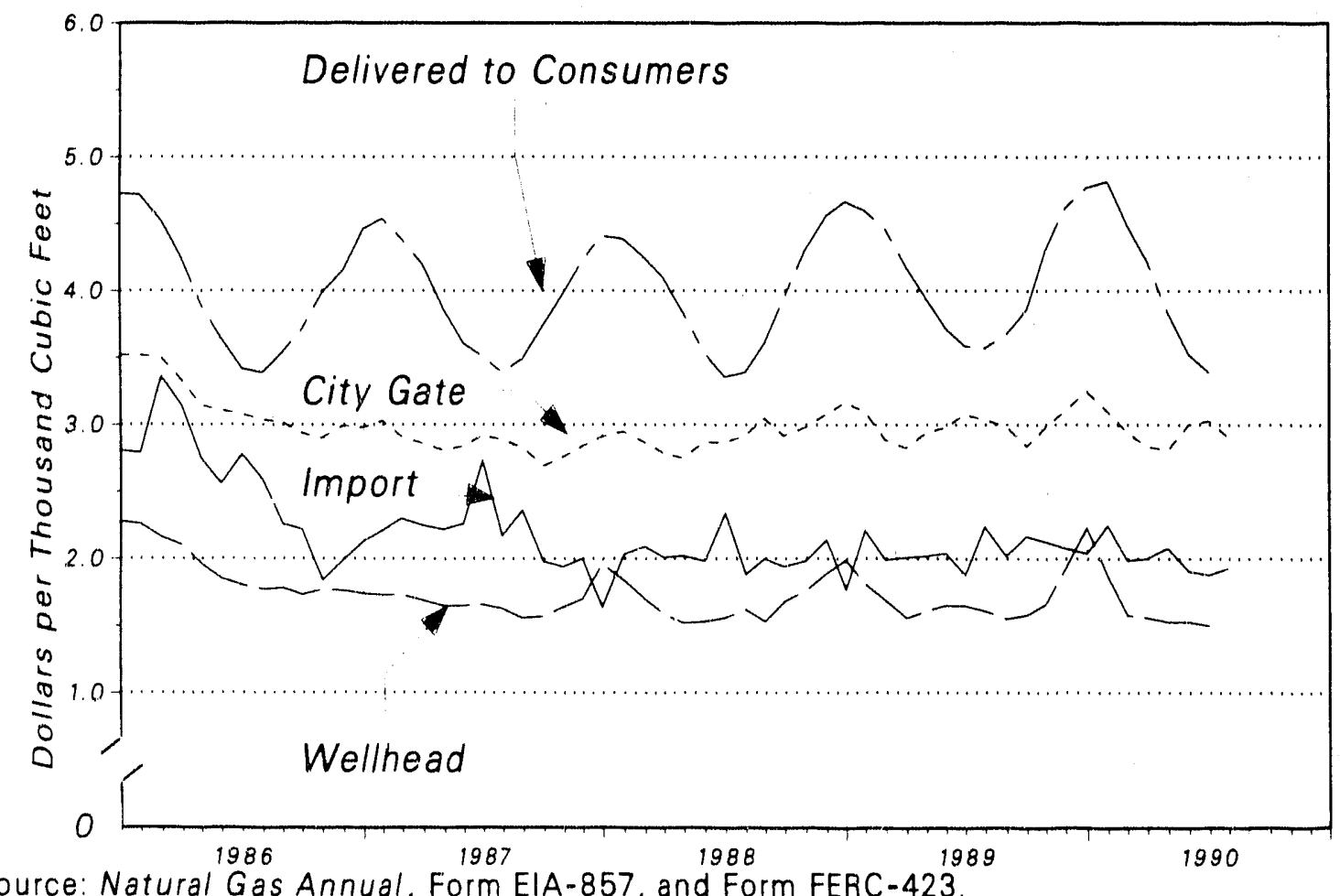

Source: Natural Gas Annual, Form ElA-857, and Form FERC -423. 
Table 5. Projected Volumes and Prices of Wellhead Purchases by NGPA Category

(Vnlımes in Billion Cubic Feet, Prices in Dollars per Thousand Cubic Feet)

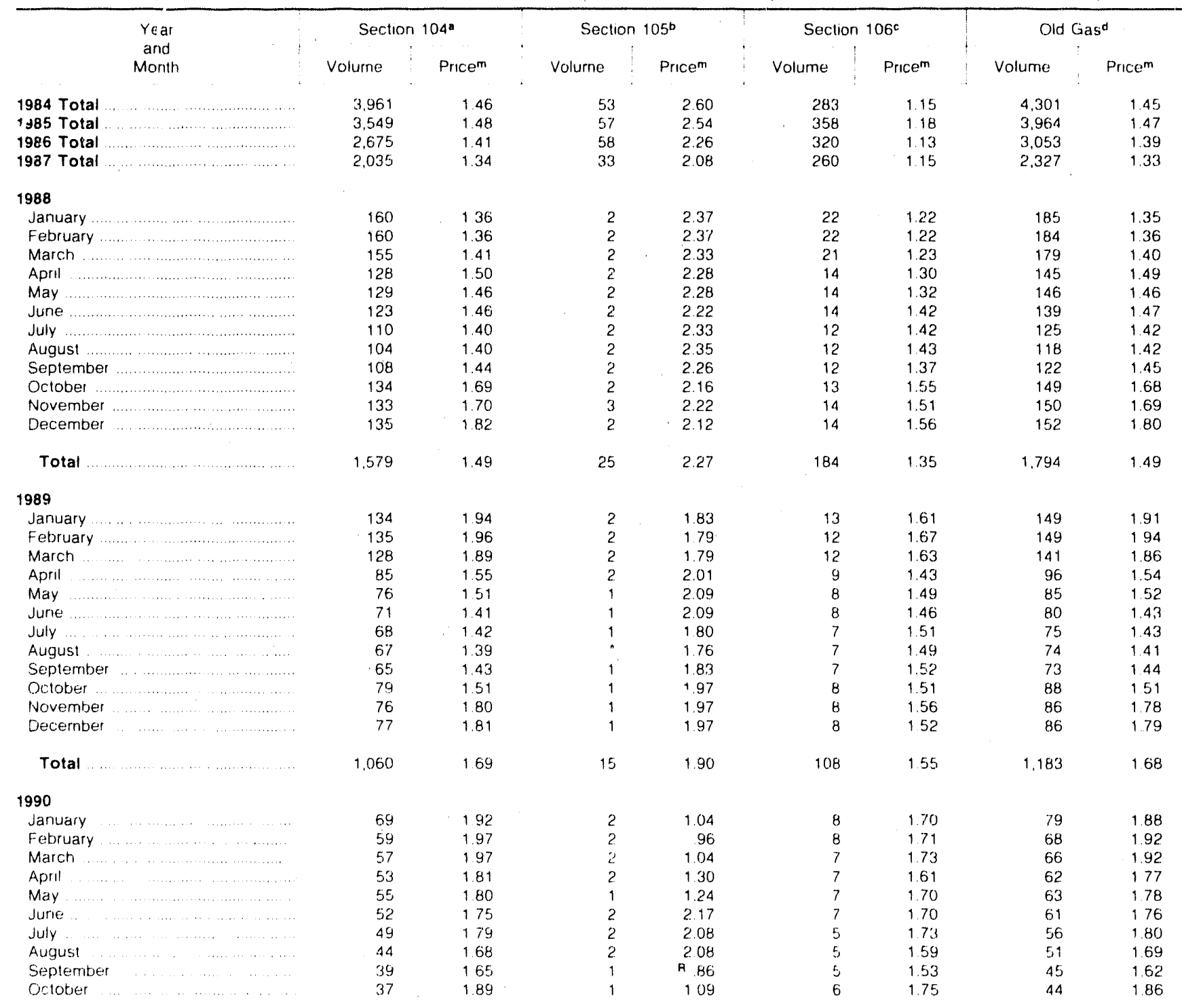

See footnotes al end of table 
Table 5. Projected Volumes and Prices of Wellhead Purchases by NGPA Category (Continued)

(Volumes in Billion Cubic Feet, Frices in Dollars per Thousand Cubic Feet)

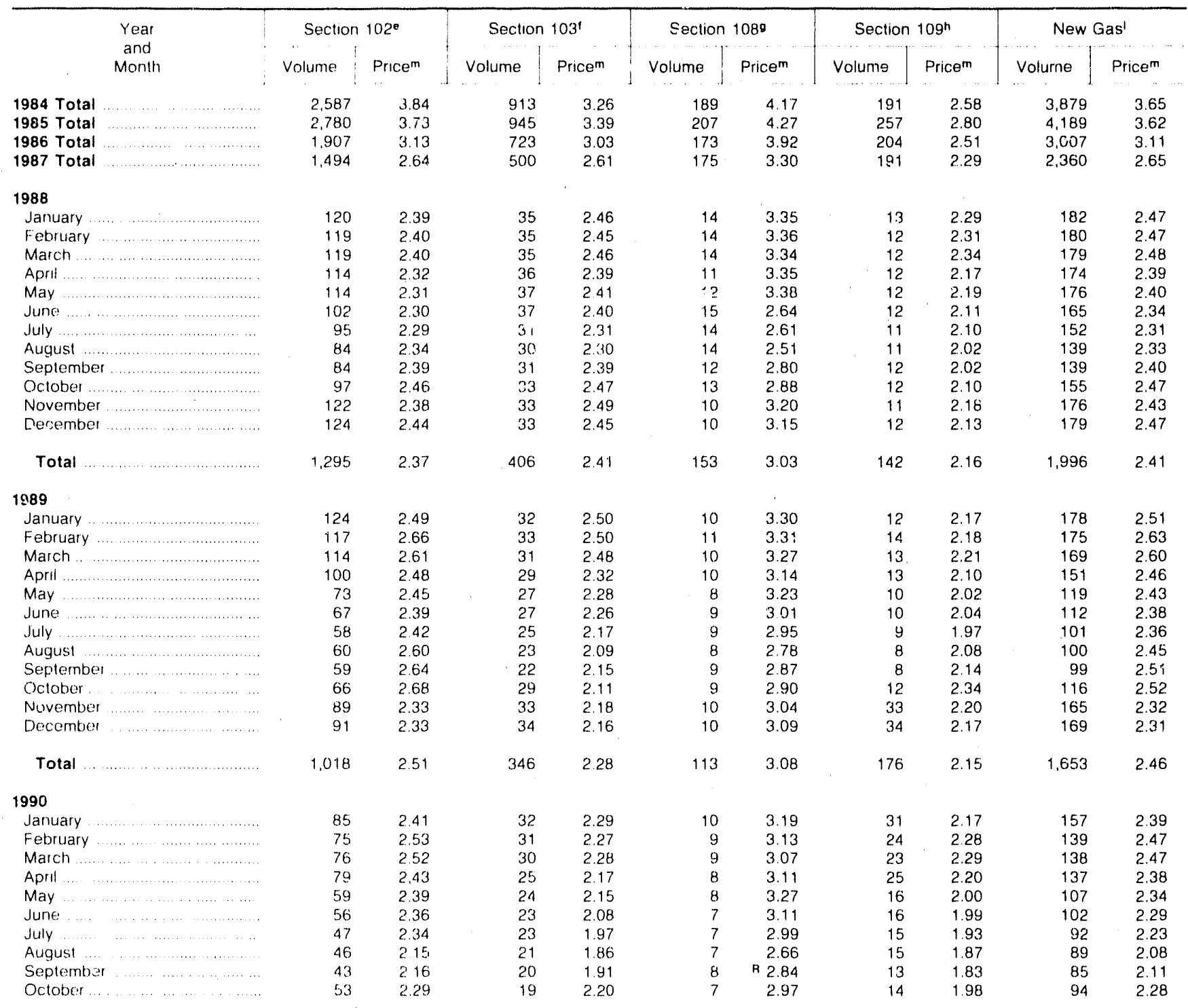

See footnotes at end of table 

Table 5. Projected Volumes and Prices of Wellhead Purchases by
NGPA Category (Continued)

(Volumes in Billion Cubic Feet, Prices in Dollars per Thousand Cubic Feet)

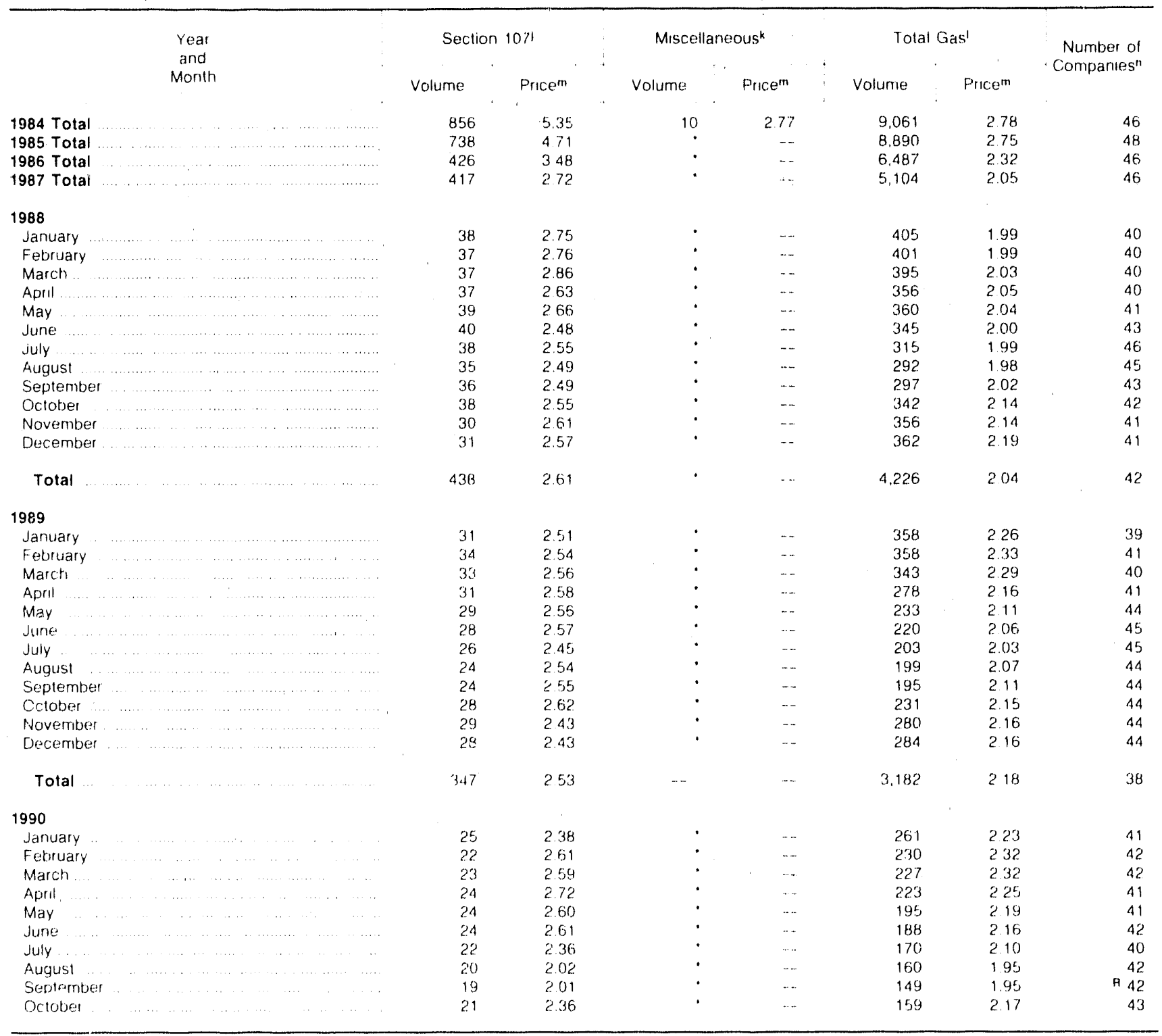

a Dedicated to iriterstate commerce.

- Existing initrastate contracts

i Rollover contracts

- Total of Sections 104, 105, and 106

- New natural gas and certam natural gas produced from the Outer Continental Shelf

1 ivew onshore production wolls

g Strpper well ratural gas

" Other categories of natural gas

- Total of Sictions 102. 103,108 , and 109

1 Hich cost natural gas.

* Natural gas not ideritfied by category in Purchased (as Adjustments (PGiA) fling

1 Total of oid gas, new gas thgh cost gas and muscellaneeus

" All prices are wrighted averages.

"Additional intersiate pipetine companes have been included with the 20 majo interstate pipetines starting wilh Pria lihngs eftective July 1 . 1984 Ser: Appendix A, Explanatory Wote 12 for expanded pipeline company list

h Revised Data

No... Noplicable

- Volume is less than sol million cubic teret.

Wotes and Sources see the last page of this section 
Figure 5. Purchased Gas Wellhead Prices

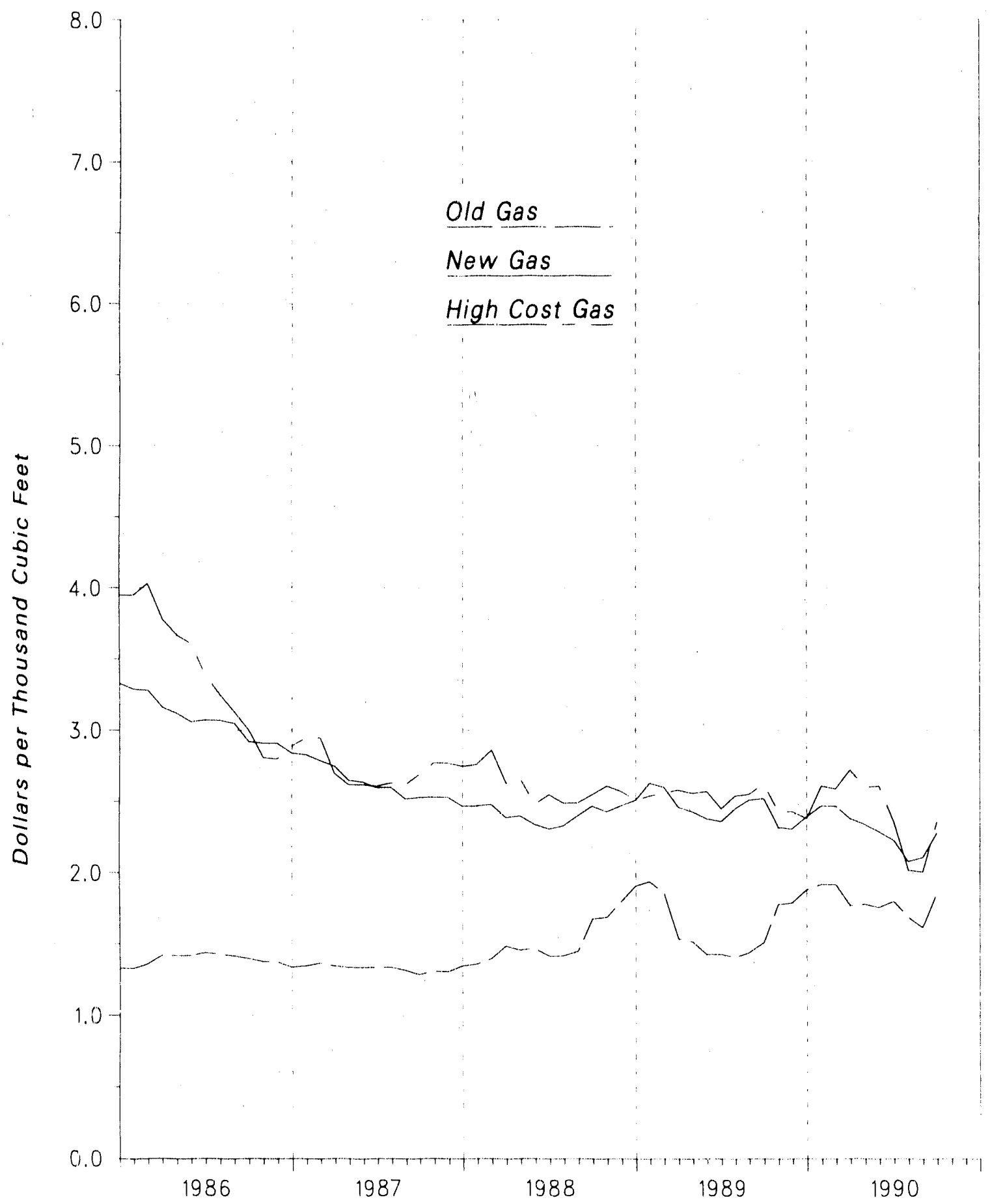

Source: Purchased Gas Adjustment filings for interstate pipeline companies. 
Table 6. Summary of Natural Gas Imports and Exports (Volumes in Million Cubic Feet, Prices in Dollars per Thousand Cubic Feet)

\begin{tabular}{|c|c|c|c|c|c|c|}
\hline \multirow{5}{*}{$\begin{array}{l}\text { Year } \\
\text { and } \\
\text { Month }\end{array}$} & \multicolumn{4}{|c|}{ Imporls } & \multicolumn{2}{|c|}{ Exports } \\
\hline & \multirow{2}{*}{\multicolumn{2}{|c|}{ Longterm Contracts }} & \multirow{2}{*}{\multicolumn{2}{|c|}{ Spot Markets }} & \multirow{4}{*}{ Volume } & \multirow{4}{*}{ Average Prico } \\
\hline & & & & & & \\
\hline & & & 1 & & & \\
\hline & Volume & Average Price & Volume & Average Price & & \\
\hline 1985 Total & 949,715 & 3.21 & $N A_{1}$ & NA & 55,268 & 4.77 \\
\hline 1986 Total $\ldots \ldots \ldots$ & 715.960 & 2.43 & 34,489 & 1.57 & 61,271 & 2.81 \\
\hline 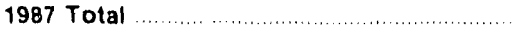 & 804,496 & 1.95 & 188,036 & 1.30 & 54,020 & 3.07 \\
\hline \multicolumn{7}{|l|}{1988} \\
\hline 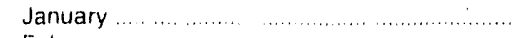 & 109,709 & 2.05 & 28,156 & 1.63 & 4,765 & 3.11 \\
\hline 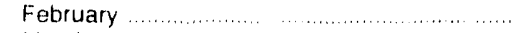 & 85,449 & 1.99 & 30,774 & 1.63 & 4,650 & 3.14 \\
\hline 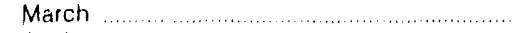 & 78,548 & 1.97 & 33,268 & 1.49 & 6,218 & 2.84 \\
\hline April $\ldots \ldots \ldots$ & 66,170 & 1.96 & 28,552 & 1.29 & 6,354 & 275 \\
\hline May $\ldots \ldots \ldots \ldots \ldots$ & 68,642 & 1.89 & 24,728 & 1.21 & 3,777 & 2.30 \\
\hline 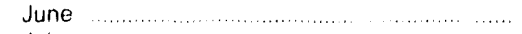 & 67,847 & 1.86 & 25,318 & 1.20 & 8,469 & 2.53 \\
\hline 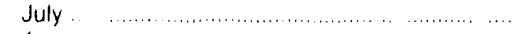 & 76,951 & 1.84 & 22,893 & 1.24 & 4,892 & 2.94 \\
\hline August $\ldots \ldots \ldots$ & 77,121 & 1.91 & 18,313 & 1.27 & 6,008 & 2.70 \\
\hline September & 75,942 & 1.92 & 19,919 & 1.35 & 7,187 & 2.59 \\
\hline October & 77,011 & 1.06 & 29,873 & 1.43 & 5,497 & 2.78 \\
\hline 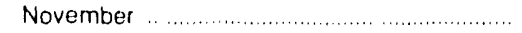 & 82,395 & 1.88 & 38,290 & 1.40 & 6,771 & 2.61 \\
\hline 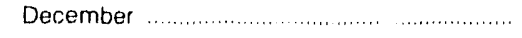 & 86,735 & 2.09 & 41,208 & 1.57 & 9,050 & 2.70 \\
\hline 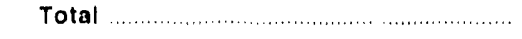 & 952,520 & 1.96 & 341,292 & 1.41 & 73,638 & 2.74 \\
\hline \multicolumn{7}{|l|}{1989} \\
\hline January ................ & 82,813 & 2.23 & 39,604 & 1.69 & 6,722 & 2.69 \\
\hline 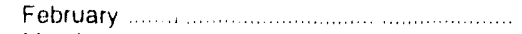 & 71,892 & 2.11 & 34,673 & 1.53 & 7,253 & 2.41 \\
\hline 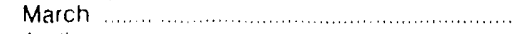 & 82,655 & 2.04 & 35,016 & 1.38 & 11.127 & 2.41 \\
\hline April $\ldots \ldots \ldots \ldots$ & 74,433 & 2.08 & 38,611 & 1.32 & 11,137 & 2.34 \\
\hline May $\ldots \ldots \ldots \ldots \ldots$ & 68,812 & 2.02 & 36,713 & 1.37 & 8,030 & 2.44 \\
\hline 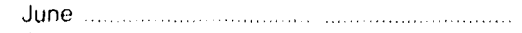 & 69,355 & 2.01 & 35,343 & 1.35 & 8.943 & 2.57 \\
\hline 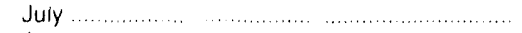 & 67,405 & 1.94 & 34,225 & 1.31 & 8,681 & 2.55 \\
\hline August & 73,268 & 2.29 & 32.701 & 1.29 & 9,148 & 2.48 \\
\hline Seplember ........... & 80,435 & 2.09 & 34,566 & 1.22 & 8,998 & 2.70 \\
\hline 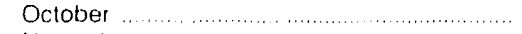 & 83,324 & 2.22 & 37,676 & 1.24 & 10,453 & 2.62 \\
\hline 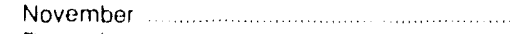 & 80,124 & 2.08 & 41,876 & 1.48 & 7,909 & 2.71 \\
\hline December & 98,801 & 2.06 & 47,199 & 1.70 & 8.471 & 2.74 \\
\hline 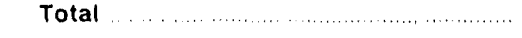 & 933,317 & 2.11 & 448,203 & 1.41 & 106,871 & 2.51 \\
\hline \multicolumn{7}{|l|}{1990} \\
\hline 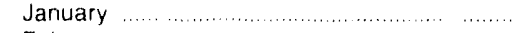 & 113,386 & 2.05 & 35,675 & 2.01 & 8,207 & 292 \\
\hline 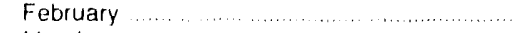 & 86,212 & 2.28 & 30,872 & 1.76 & 7,933 & 2.71 \\
\hline 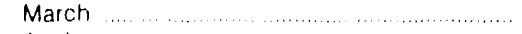 & 88,372 & 2.24 & 27.854 & 1.68 & 9,846 & 2.49 \\
\hline 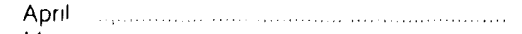 & 76,113 & 2.22 & 36.281 & 1.50 & 8.124 & 2.48 \\
\hline 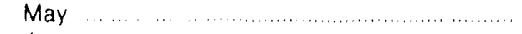 & 69,224 & 2.19 & 33,692 & 1.48 & 7,833 & 2.51 \\
\hline June & 68,667 & 2.21 & 31,504 & 1.41 & 8.617 & 2.58 \\
\hline
\end{tabular}

NA Nol Avallabl:

Notes and Sources: See the last page of this section. 
Table 7. Marketed Production of Natural Gas by State (Million Cubic Feet)

\begin{tabular}{|c|c|c|c|c|c|c|c|}
\hline Year and Month & Alabarna & Alatska & Arkanlsas & Califomia & Colorado & Florida & Kansas \\
\hline 1984 Total & 101,821 & 284,129 & 135,161 & 476,333 & 173,257 & 12.6815 & 465,979 \\
\hline 1985 Total & 107.342 & 321,346 & 155,099 & 491.283 & 178,233 & 10,545 & 512,872 . \\
\hline 1986 Total. & 107,1834 & 304,841 & 131,075 & 462,218 & 163,684 & 8,833 & 465,695 \\
\hline 1987 Total. & 117,241 & 359,837 & 141,151 & 424,621 & 164,557 & 8,281 & 457,050 \\
\hline \multicolumn{8}{|l|}{1988} \\
\hline 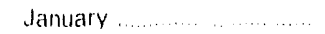 & 11,392 & 32,910 & 19,390 & 34,672 & 19,577 & 652 & 72,587 \\
\hline Fobruary ............ & 10,929 & 29,964 & 15,308 & 30,938 & 18,155 & 597 & 50,850 \\
\hline$\therefore \ldots \ldots+\ldots \ldots \ldots$ & 11,255 & 36,393 & 14,968 & 35,114 & 18,025 & 643 & 55,095 \\
\hline$\ldots \ldots \ldots \ldots$ & 10,471 & 29,666 & 12,473 & 32,892 & 15,972 & 614 & 46,812 \\
\hline 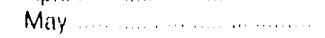 & 9,236 & 28,656 & 12,473 & 34,562 & 16,515 & 607 & 13,514 \\
\hline June $\ldots \ldots \ldots \ldots \ldots \ldots \ldots . . . . . . . . . . . .$. & 10,523 & 29,466 & 11,793 & 33,765 & 12,769 & 790 & 45,892 \\
\hline July ...... & 10,953 & 29,263 & 11,566 & 33,339 & 13,028 & 677 & 36,847 \\
\hline 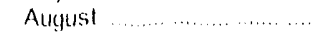 & 10,753 & 29,530 & 13,040 & 35,317 & 14,001 & 644 & 33,789 \\
\hline 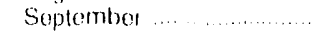 & 10,368 & 30,159 & 12,246 & 34,343 & 14,099 & 602 & 28,996 \\
\hline 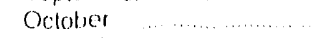 & 11.100 & 94,017 & $12,58 i$ & 33,193 & 15,057 & 538 & 46,615 \\
\hline Novembe! $\ldots . . . . . . . . . . . . . .$. & 10,663 & 34,088 & 13,607 & 30,674 & 15,588 & 528 & 51,178 \\
\hline December .............. & 11,880 & 34.525 & 17,122 & 30,855 & 18.759 & 593 & 64,090 \\
\hline . & 129,524 & 378,638 & 166,573 & 394,663 & 191,544 & 7,484 & 576,274 \\
\hline \multicolumn{8}{|l|}{1989} \\
\hline 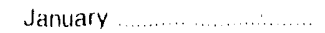 & 12,166 & 35,680 & E 20,100 & 30,377 & 22,256 & 639 & 57,650 \\
\hline 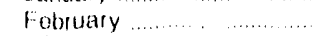 & 10,588 & 33,405 & E 15,300 & 28,008 & 19,088 & 550 & 60,563 \\
\hline 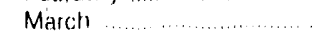 & 11,626 & 36,160 & E. 15,900 & 30,661 & 17,871 & 577 & 55,638 \\
\hline 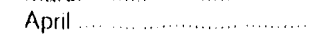 & 11,028 & 33,821 & E 12,800 & 30,396 & 15,564 & 597 & $47,09 ?$ \\
\hline 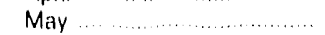 & 11,340 & 24,870 & E 12,500 & 31,068 & 16,010 & 660 & 50,559 \\
\hline June & 10,914 & 32,380 & E 11,900 & 30,379 & 15,551 & 571 & 42,180 \\
\hline 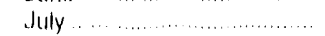 & 11,212 & 32,531 & E 11,500 & 32,013 & 15,750 & 638 & 47,756 \\
\hline 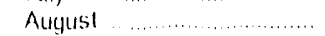 & 10,599 & 30,266 & E 13,200 & 31.718 & 15,420 & 645 & 43,662 \\
\hline 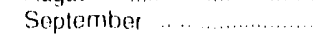 & 10,119 & 30,257 & E 12,000 & 29,806 & 10,468 & 665 & 36,719 \\
\hline October & 9,709 & 34.951 & E 12,400 & 30,677 & 19,373 & 708 & 41,051 \\
\hline Novernber & 9.137 & 34,619 & E 13,900 & 28,134 & 21,568 & 611 & 46,650 \\
\hline Decomber ................. & 9,973 & 35.789 & E 16,800 & 29,623 & 21,818 & 673 & 57.710 \\
\hline$\ldots \ldots \ldots+\ldots \ldots, \ldots \ldots$, & 128,411 & 393,729 & E 168,300 & 362,860 & 216,737 & 7.5 .34 & 587.230 \\
\hline \multicolumn{8}{|l|}{1990} \\
\hline . & 10,650 & 36,514 & E 20,800 & 28,313 & 21,484 & 699 & 57,345 \\
\hline Fobruary. & 10,097 & 34.268 & E 15,600 & 25,362 & 17,390 & 649 & 48,176 \\
\hline March & 11,223 & 34.845 & E 16,400 & 30,292 & 17,567 & 553 & 41,472 \\
\hline April & 11,165 & 32,533 & E 13,700 & 29,010 & $18,0 \% 0$ & 629 & 40,122 \\
\hline 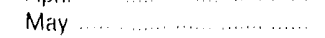 & 11,736 & 29,666 & E 13,100 & E 29,800 & 18,034 & 660 & 45,477 \\
\hline 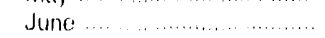 & 10,988 & 30,091 & E. 12,700 & 29,100 & 16,976 & 557 & E 10,801 \\
\hline 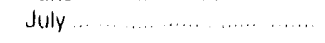 & 11,670 & 31.215 & E 12,400 & E 29,600 & 17,071 & 537 & E 40,191 \\
\hline 1990 YTD & 77,529 & 228,132 & 105,000 & 201,477 & 126,592 & 1,284 & 313,884 \\
\hline 1989 YTD & 78,874 & 227.847 & 100,000 & 212,902 & 122,090 & 4,232 & 361,438 \\
\hline$\ldots \ldots \ldots$ & 74,759 & 216,318 & 97,971 & 235,282 & 114,041 & 4,580 & 351,605 \\
\hline
\end{tabular}

See footnoles at and of tathe 
Table 7. Marketed Prodiuction of Natural Gas by State (Continued) (Million Cubic Feet)

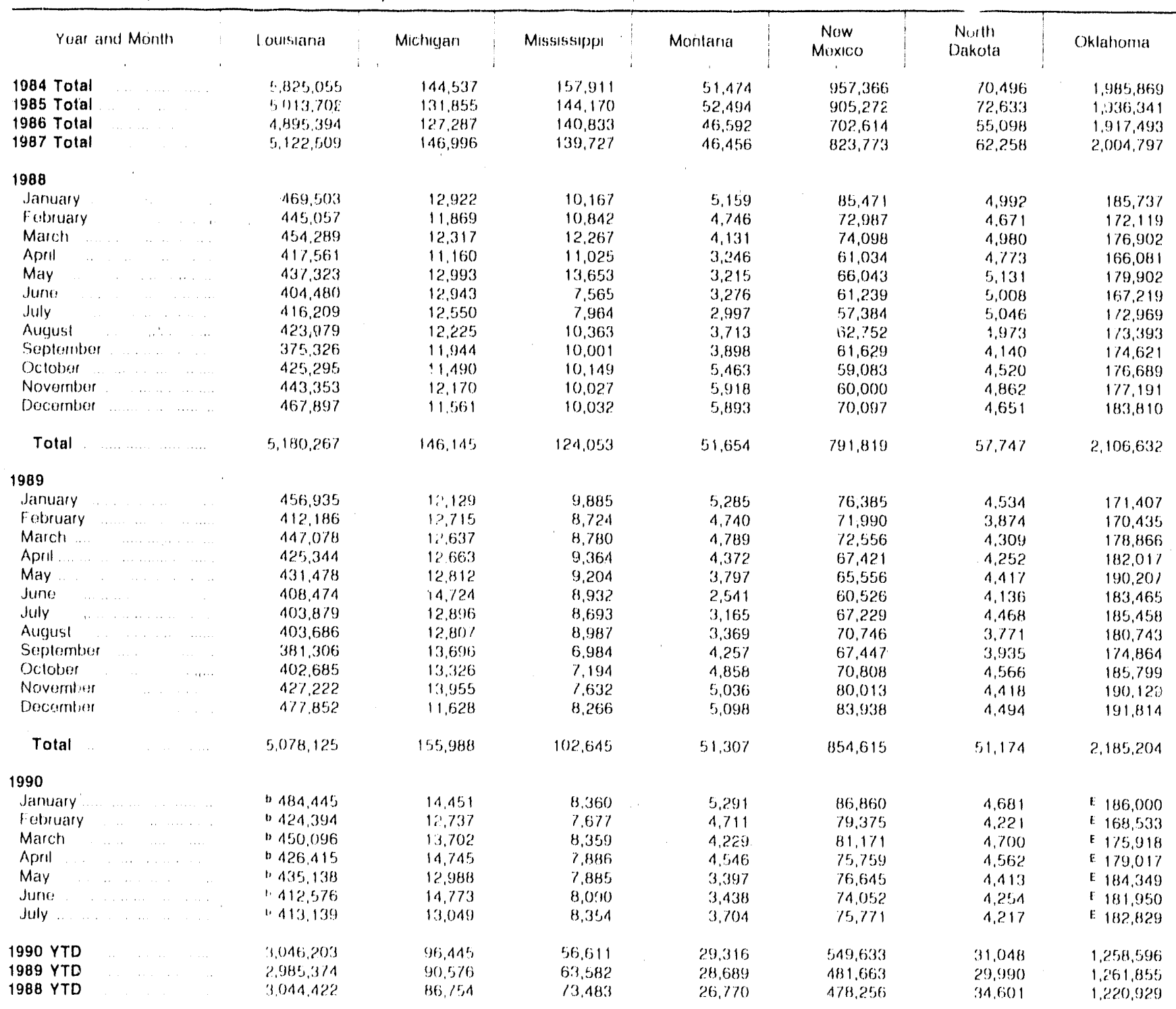

Seo lootnotes at end of lable 
Table 7. Marketed Production of Natural Gas by State (Continued) (Million Cubic Feet)

\begin{tabular}{|c|c|c|c|c|c|c|}
\hline Year and Month & Toxas & Utah & $\begin{array}{l}\text { Wosit } \\
\text { Virginia }\end{array}$ & Wyoming & $\begin{array}{l}\text { Othor" } \\
\text { Statos }\end{array}$ & $\begin{array}{l}\text { U.S. } \\
\text { Total }\end{array}$ \\
\hline $\begin{array}{l}1984 \text { Total } \\
1985 \text { Total } \\
1986 \text { Total } \\
1987 \text { Total }\end{array}$ & $\begin{array}{l}6,185,021 \\
6,052,663 \\
6,151,775 \\
6,126,315\end{array}$ & $\begin{array}{l}74.698 \\
83,405 \\
90,013 \\
87,158\end{array}$ & $\begin{array}{l}143,730 \\
144,883 \\
135,431 \\
160,000\end{array}$ & $\begin{array}{l}516,683 \\
416,565 \\
403,266 \\
497,980\end{array}$ & $\begin{array}{l}462,5333 \\
467,254 \\
481,584 \\
457,830\end{array}$ & $\begin{array}{l}18,229,638 \\
17,197,990 \\
16,790,910 \\
17,348,537\end{array}$ \\
\hline 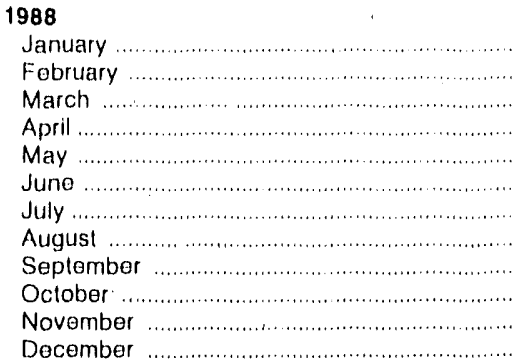 & $\begin{array}{l}575,425 \\
529,339 \\
548,365 \\
507,830 \\
514,825 \\
493,090 \\
513,708 \\
521,584 \\
484,953 \\
526,788 \\
525,427 \\
544,695\end{array}$ & $\begin{array}{r}10,268 \\
9,369 \\
9,602 \\
8,280 \\
9,197 \\
7,475 \\
7,287 \\
8,275 \\
8,562 \\
8,032 \\
10,031 \\
4,993\end{array}$ & $\begin{array}{l}17,005 \\
16,449 \\
15,227 \\
14,671 \\
14,226 \\
14,116 \\
14,004 \\
13,670 \\
13,560 \\
11,892 \\
15,005 \\
15,116\end{array}$ & $\begin{array}{l}48,751 \\
34,516 \\
50,106 \\
48,261 \\
35,440 \\
43,307 \\
37,103 \\
29,618 \\
42,143 \\
43,810 \\
45,919 \\
50,085\end{array}$ & $\begin{array}{l}40,008 \\
39,504 \\
39,605 \\
37,589 \\
37,589 \\
36,884 \\
37,388 \\
38,698 \\
37,186 \\
39,705 \\
38,295 \\
39,605\end{array}$ & $\begin{array}{l}1,656,588 \\
1,508,217 \\
1,573,382 \\
1,440,411 \\
1,475,100 \\
1,401,600 \\
1,420,282 \\
1,440,317 \\
1,358,776 \\
1,476,023 \\
1,504,524 \\
1,586,259\end{array}$ \\
\hline Total $\ldots \ldots \ldots \ldots \ldots$ & $6,286,029$ & 101,372 & 174,942 & 509,058 & 462,056 & $17,841,474$ \\
\hline 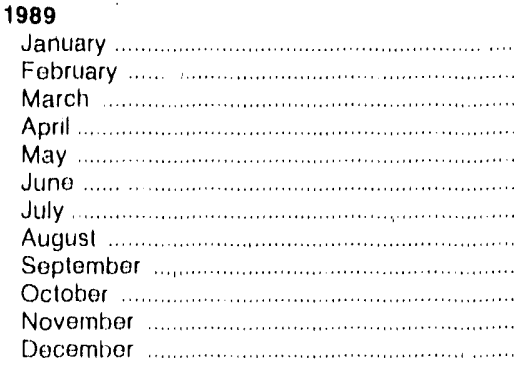 & $\begin{array}{l}545,995 \\
498,336 \\
541,753 \\
518,207 \\
525,157 \\
508,113 \\
520,968 \\
516,596 \\
491,862 \\
512,554 \\
517,874 \\
544,010\end{array}$ & $\begin{array}{r}11,563 \\
10,631 \\
10,346 \\
9,313 \\
10,134 \\
7,824 \\
10,092 \\
9,588 \\
8,462 \\
9,473 \\
10,130 \\
12,533\end{array}$ & $\begin{array}{ll}\text { E. } & 20,100 \\
\text { E } & 19,700 \\
\text { E } & 18,200 \\
\text { E } & 16,700 \\
\text { E } & 15,900 \\
\text { E } & 16,600 \\
\text { E } & 15,300 \\
E & 14,900 \\
\text { E } & 14,500 \\
E & 12,700 \\
\text { E } & 16,400 \\
\text { I: } & 17,200\end{array}$ & $\begin{array}{l}63,140 \\
54,794 \\
56,294 \\
51,686 \\
56,519 \\
52,138 \\
51,594 \\
50,036 \\
51,023 \\
55,332 \\
58,195 \\
64,948\end{array}$ & $\begin{array}{l}41,235 \\
40,722 \\
40,824 \\
38,773 \\
38,978 \\
37,850 \\
38,363 \\
39,696 \\
38,158 \\
40,722 \\
39,286 \\
40,620\end{array}$ & $\begin{array}{l}1,597,461 \\
1,476,349 \\
1,563,865 \\
1,491,410 \\
1,511,166 \\
1,449,198 \\
1,473,505 \\
1,460,435 \\
1,392,528 \\
1,468,886 \\
1,524,909 \\
1,634,787\end{array}$ \\
\hline Total & $6,241,425$ & 120,089 & E. 198,200 & 665,699 & 475,227 & $18,044,499$ \\
\hline 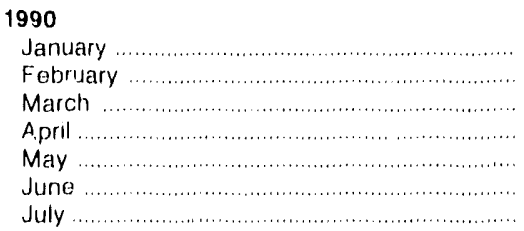 & $\begin{array}{r}570,208 \\
\text { c } 506,557 \\
541,062 \\
513,659 \\
527,747 \\
513,071 \\
502,333\end{array}$ & $\begin{array}{r}14,705 \\
12,782 \\
12,822 \\
9,629 \\
11,992 \\
12,060 \\
11,396\end{array}$ & $\begin{array}{l}\text { E } 23,000 \\
\text { E } 22,600 \\
\text { Е } 20,100 \\
\text { E } 19,700 \\
\text { E } 17,600 \\
\text { E } 18,100 \\
\text { E } 17,100\end{array}$ & $\begin{array}{l}6,1,622 \\
56,233 \\
62,938 \\
56,500 \\
5,4,733 \\
48,413 \\
55,332\end{array}$ & $\begin{array}{l}\text { E } 41,100 \\
\text { E } 40,600 \\
\text { E } 40,700 \\
\text { E } 38,700 \\
\text { E } 37,400 \\
\text { E } 36,400 \\
\text { Е } 36,900\end{array}$ & $\begin{array}{l}1,675,528 \\
1,491,962 \\
1,568,149 \\
1,496,647 \\
1,523,060 \\
1,468,390 \\
1,466,808\end{array}$ \\
\hline $\begin{array}{l}1990 \text { YTD } \\
1989 \text { YTD } \\
1988 \text { YTD }\end{array}$ & $\begin{array}{l}3,674,637 \\
3,658,529 \\
3,682,582\end{array}$ & $\begin{array}{l}85,386 \\
69,903 \\
61,478\end{array}$ & $\begin{array}{l}138,200 \\
12,2,500 \\
105,698\end{array}$ & $\begin{array}{l}395,771 \\
386,165 \\
297,484\end{array}$ & $\begin{array}{l}271,800 \\
276,7145 \\
268,567\end{array}$ & $\begin{array}{l}10,690,544 \\
10,562,454 \\
10,475,580\end{array}$ \\
\hline
\end{tabular}

a Includes Arizona, Illinois, Indiana. Kontucky, Maryland, Missouri, Nebraska, Now York, Ohio, Oregon, Ponnsiylvana, South Dakota, Tonnosseo, and Virginia. The 1990 monthly values for thess Statos are estimated.

b Total marketed production for Louisiana in January - July 1990 were ostimated on the basis of production traported in January - Decombor 1989, soasonally adjusted.

c Total markoted production for Texas in February 1990 roprosents roportod data lor Stalo onshoro and ostimated data for Outer Contınental Shelf

(OCS) producing aroas.
Estumated Data

Notes and Sourcers: Soe the last paye of this section. 
Table 8. Well Determination Filings (Volume in Billion Cubic Feet)

\begin{tabular}{|c|c|c|c|c|c|c|c|c|c|}
\hline \multirow{3}{*}{$\begin{array}{c}\text { Year } \\
\text { and } \\
\text { Month }\end{array}$} & \multicolumn{3}{|c|}{ Section $102^{a}$} & \multicolumn{3}{|c|}{ Section $103^{b}$} & \multicolumn{3}{|c|}{ Section $107^{\circ}$} \\
\hline & \multicolumn{2}{|c|}{ Number of Filingse } & t & \multicolumn{2}{|c|}{ Number of Filingse } & \multirow[b]{2}{*}{$\begin{array}{l}\text { Estimated } \\
\text { Annual } \\
\text { Volume' }\end{array}$} & \multicolumn{2}{|c|}{ Number of Filingse } & \multirow[b]{2}{*}{$\begin{array}{c}\text { Estimated } \\
\text { Annual } \\
\text { Volume' }\end{array}$} \\
\hline & Total & $\begin{array}{c}\text { With } \\
\text { Volumes }\end{array}$ & $\begin{array}{c}\text { Estimated } \\
\text { Annual } \\
\text { Volumo' }\end{array}$ & Total & $\begin{array}{l}\text { With } \\
\text { Volumes }\end{array}$ & & Total & $\begin{array}{l}\text { With } \\
\text { Volumos }\end{array}$ & \\
\hline 1984 Total .............. & 10,167 & 8,071 & 2,432 & 16,048 & 12,751 & 1,217 & 9,656 & 7,846 & 757 \\
\hline i985 Total .................. & 9.052 & 7,028 & 1,905 & 15,896 & 12,531 & 1,455 & 10,940 & 8,766 & 7.41 \\
\hline 1986 Total .................... & 4,631 & 3,620 & 1,010 & 11,348 & 8,956 & 959 & 6,709 & 4,874 & 3137 \\
\hline 1987 Total .................. & 2.387 & 1,787 & 471 & 5,717 & 4,548 & 532 & 6,655 & 4,242 & 248 \\
\hline \multicolumn{10}{|l|}{1988} \\
\hline January ............................ & 118 & 86 & 18 & 444 & 352 & 32 & 327 & 233 & 13 \\
\hline February .................. & 186 & 132 & 31 & 540 & 415 & 44 & 605 & 386 & 21 \\
\hline March & 204 & 135 & 38 & 670 & 495 & 82 & 582 & 341 & 14 \\
\hline 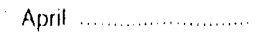 & 101 & 80 & 18 & 686 & 477 & 59 & 454 & 232 & 14 \\
\hline 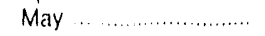 & 170 & 127 & 30 & 680 & 571 & 66 & 620 & 426 & 18 \\
\hline 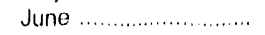 & 425 & 360 & 55 & 794 & 698 & 40 & 444 & 252 & 13 \\
\hline July ........................... & 121 & 7 & 22 & 269 & 198 & 21 & 478 & 304 & 19 \\
\hline 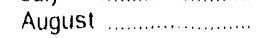 & 190 & $1: 5$ & 217 & 585 & 458 & 51 & 575 & 377 & 25 \\
\hline Seplember .................. & 111 & 83 & 23 & 435 & 349 & 54 & 535 & 246 & 17 \\
\hline 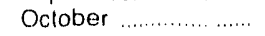 & 193 & 104 & 27 & 356 & 288 & 25 & 465 & 288 & 10 \\
\hline 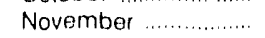 & 115 & 90 & 26 & 345 & 271 & 30 & 269 & 144 & 7 \\
\hline December & 183 & 105 & 21 & 555 & 432 & 60 & 389 & 265 & 16 \\
\hline 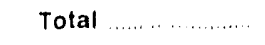 & 2,147 & 1,508 & 526 & 6,359 & 5,004 & 563 & 5,743 & 3,494 & 185 \\
\hline \multicolumn{10}{|l|}{1989} \\
\hline 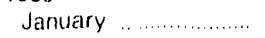 & 124 & 97 & 25 & 389 & 300 & 31 & 385 & 286 & 14 \\
\hline 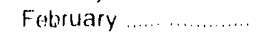 & 134 & 103 & 28 & 400 & 303 & 54 & 268 & 219 & 14 \\
\hline March & 104 & 78 & 22 & 392 & 317 & 23 & 351 & 234 & 10 \\
\hline 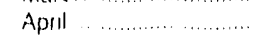 & 131 & 93 & 24 & 384 & 279 & 40 & 286 & 217 & 15 \\
\hline 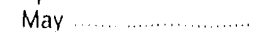 & 135 & 107 & 29 & 405 & 285 & 30 & 295 & 192. & 21 \\
\hline June & 56 & 44 & 13 & 294 & 199 & 18 & 214 & 136 & 6 \\
\hline 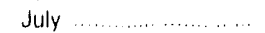 & 137 & 110 & 24 & $46 ?$ & 339 & 38 & 266 & 218 & 14 \\
\hline August $\ldots \ldots \ldots$ & 124 & 91 & 26 & 313 & 236 & 24 & 341 & 259 & 28 \\
\hline 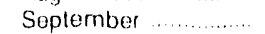 & 74 & 61 & 102 & 269 & 195 & 32 & 314 & 268 & 17 \\
\hline October & 87 & 73 & 24 & 448 & 338 & 57 & 385 & 293 & 20 \\
\hline November ............... & 91 & 69 & 21 & 233 & 143 & 20 & 364 & 302 & 22 \\
\hline Decernber .................. & 55 & 41 & 7 & 205 & 126 & 13 & 362 & 229 & 14 \\
\hline Total ...................... & 1.262 & 967 & 344 & 4,194 & 3,060 & 379 & 3,831 & 2,853 & 195 \\
\hline \multicolumn{10}{|l|}{1990} \\
\hline January ..................... & 85 & 67 & 17 & 289 & 173 & 24 & 388 & 298 & 21 \\
\hline Fobruary & 67 & 54 & 20 & 248 & 155 & 18 & 389 & 262 & 16 \\
\hline 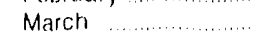 & 122 & 86 & 17 & 291 & 181 & 17 & 714 & 168 & 29 \\
\hline April & 93 & 82 & 18 & 204 & 137 & 11 & 425 & 231 & 22 \\
\hline 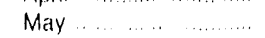 & 82 & 71 & 17 & 367 & 213 & 26 & 553 & 378 & 32 \\
\hline June & 45 & 39 & 10 & 297 & 224 & 22 & 468 & 349 & 42 \\
\hline July & 34 & 28 & 7 & 202 & 144 & 20 & 362 & 254 & 21 \\
\hline August & 52 & 29 & 13 & 205 & 139 & 24 & 359 & 221 & 13 \\
\hline 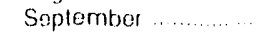 & 58 & 43 & 19 & 208 & 138 & 21 & 493 & 388 & 27 \\
\hline 1990 YTD & 638 & $49 ?$ & 136 & 2,311 & 1,504 & 182 & 4,151 & 2,849 & 224 \\
\hline 1989 YTD & 1,029 & 784 & 292 & 3,308 & 2,453 & 289 & 2,720 & 2,029 & 139 \\
\hline 1988 YTD & 1.626 & 1,209 & 452 & 5,103 & 4.013 & 449 & 4,620 & 2,797 & 153 \\
\hline
\end{tabular}

See footnotos at end of table 
Table 8. Well Determination Filings (Continued) (Volume in Billion Cubic Feet)

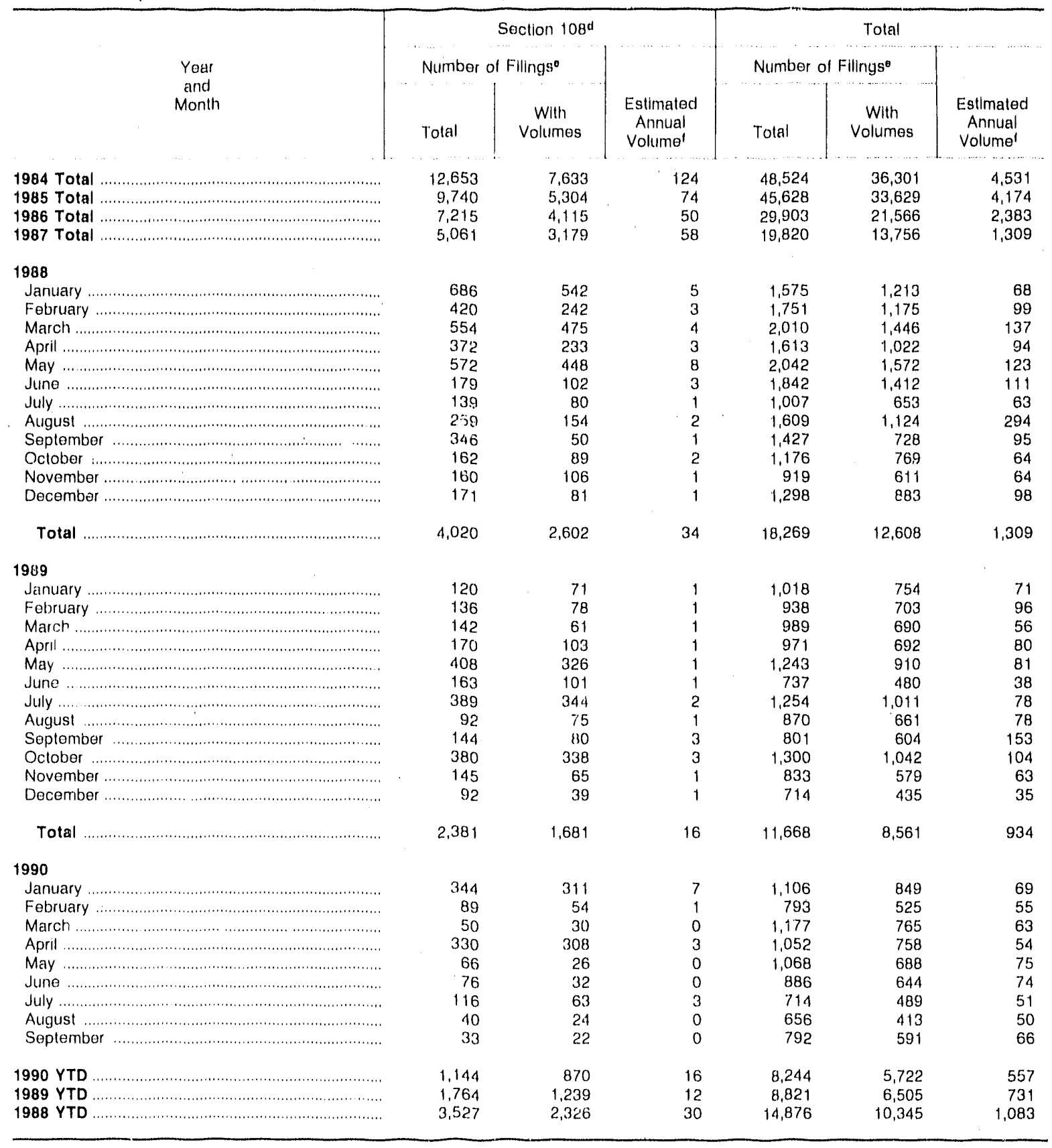

a New natural gas and certain natural gas produced from the Outer Continental Shelf.

b New onshore production wells.

c High cost natural gas.

d Stripper well natural gas.

- Nol all filings report estimated volumes. The "With Volumes" columns show number reporting volumes

1 Annual volumes are often estimated by producers prior lo actual operations or based on short periods of operation. For this reason the accuracy of estimates may vary.

Notes and Sources: See the last page of this section. 
Table 9. Well Determination Filings by State, September 1990 (Volume in Million Cubic Feet)

\begin{tabular}{|c|c|c|c|c|c|c|c|c|c|}
\hline \multirow{3}{*}{ State } & \multicolumn{3}{|c|}{ Section $102^{a}$} & \multicolumn{3}{|c|}{ Section $103^{b}$} & \multicolumn{3}{|c|}{ Section $107^{\circ}$} \\
\hline & Number & Filings ${ }^{\circ}$ & & Number & Filings" & & Number & Ellings $s^{\theta}$ & \\
\hline & Total & $\begin{array}{c}\text { With } \\
\text { Volumes }\end{array}$ & $\begin{array}{l}\text { Estimated } \\
\text { Annual } \\
\text { Volume' }\end{array}$ & Tolal & $\begin{array}{c}\text { With } \\
\text { Volumes }\end{array}$ & $\begin{array}{l}\text { Estimated } \\
\text { Arinual } \\
\text { Volume }\end{array}$ & Total & $\begin{array}{l}\text { With } \\
\text { volumes }\end{array}$ & $\begin{array}{l}\text { Estimaled } \\
\text { Annual } \\
\text { Volume }\end{array}$ \\
\hline Alabama ..................... & 3 & 3 & 610 & 0 & 0 & 0 & 124 & 123 & 7,141 \\
\hline Arkansas & 24 & 21 & 10,062 & 37 & 36 & 5,638 & 1 & 0 & 0 \\
\hline California ....................... & 2 & 0 & 0 & 0 & 0 & 0 & 0 & 0 & 0 \\
\hline Colorado & 0 & 0 & 0 & 0 & 0 & 0 & 5 & 4 & 300 \\
\hline |ll|nois $\ldots \ldots \ldots \ldots$ & 0 & 0 & 0 & 0 & 0 & 0 & 6 & 6 & 287 \\
\hline Kansas $\ldots \ldots \ldots \ldots \ldots \ldots \ldots$ & 4 & 1 & 180 & 11 & 10 & 1,126 & 2 & 2 & 170 \\
\hline Kentucky ......................... & 0 & 0 & 0 & 26 & 10 & 203 & 41 & 38 & 1,422 \\
\hline Louisiana ...................... & 1.3 & 12 & 4,058 & 13 & 11 & 1,961 & 0 & 0 & 0 \\
\hline Michigan ........................ & 0 & 0 & 0 & 0 & 0 & 0 & 93 & 67 & 1.743 \\
\hline Mississippi ..................... & 0 & 0 & 0 & 5 & 5 & 794 & 5 & 3 & 6 \\
\hline Montana ........................ & 0 & 0 & 0 & 0 & 0 & 0 & 0 & 0 & 0 \\
\hline New Mexico ................. & 0 & 0 & 0 & 3 & 3 & 1,000 & 40 & 24 & 9,911 \\
\hline New York ................... & 0 & 0 & 0 & 10 & 0 & 0 & 1 & 0 & 0 \\
\hline 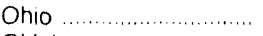 & 0 & 0 & 0 & 4 & 3 & 291 & 31 & 21 & 451 \\
\hline 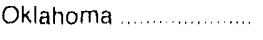 & 2 & 0 & 0 & 52 & 31 & 7,527 & 0 & 0 & 0 \\
\hline Pennsylvania ................. & 0 & 0 & 0 & 11 & 7 & 205 & 25 & 25 & 526 \\
\hline 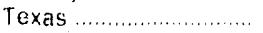 & 10 & 6 & 3,605 & 28 & 17 & 1,846 & 39 & 1.3 & 3,619 \\
\hline Wesi Virginia ................. & 0 & 0 & 0 & 8 & 5 & 161 & 79 & 61 & 1,163 \\
\hline Wyomirig ....................... & 0 & 0 & 0 & 0 & 0 & 0 & 1 & 1 & 90 \\
\hline 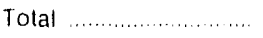 & 58 & 43 & 18,515 & 208 & 138 & 20,752 & 493 & 388 & 26,829 \\
\hline
\end{tabular}

See lootnotes at end of table. 
Table 9. Well Determination Filings by State, September 1990 (Continued) (Volume in Million Cubic Feet)

\begin{tabular}{|c|c|c|c|c|c|c|}
\hline \multirow{3}{*}{ State } & \multicolumn{3}{|c|}{ Section $108^{d}$} & \multicolumn{3}{|c|}{ Total } \\
\hline & \multicolumn{2}{|c|}{ Number of Filings ${ }^{e}$} & \multirow[b]{2}{*}{$\begin{array}{l}\text { Estimated } \\
\text { Annual } \\
\text { Volume' }\end{array}$} & \multicolumn{2}{|c|}{ Number of Filingse } & \multirow[b]{2}{*}{$\begin{array}{c}\text { Estimated } \\
\text { Annual } \\
\text { Volume' }\end{array}$} \\
\hline & Total & $\begin{array}{l}\text { With } \\
\text { Volumes }\end{array}$ & & Total & $\begin{array}{l}\text { With } \\
\text { Volumes }\end{array}$ & \\
\hline Alabama & 0 & 0 & 0 & 127 & 126 & 7,751 \\
\hline 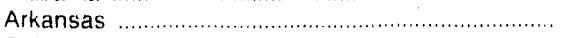 & 0 & 0 & 0 & 62 & 57 & 15,700 \\
\hline California & 0 & 0 & 0 & 2 & 0 & 0 \\
\hline 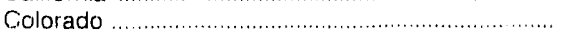 & 0 & 0 & 0 & 5 & 4 & 300 \\
\hline 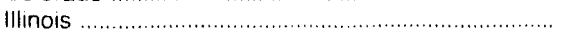 & 0 & 0 & $\checkmark$ & 6 & 6 & 287 \\
\hline 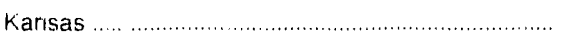 & 2 & 0 & 0 & 19 & $13^{\prime}$ & 1,476 \\
\hline 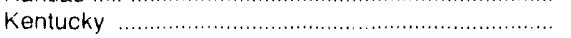 & 0 & 0 & 0 & 67 & 48 & 1,625 \\
\hline 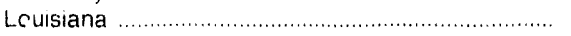 & 1 & 0 & 0 & 27 & 23 & 6,019 \\
\hline 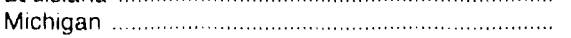 & 0 & 0 & 0 & S., & 67 & 1,743 \\
\hline 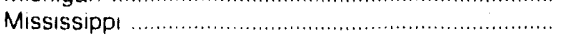 & 0 & 0 & 0 & 10 & 8 & 800 \\
\hline Montana & 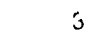 & 0 & 0 & 3 & 0 & 0 \\
\hline New Mexico & 0 & 0 & 0 & 43 & 27 & 10,911 \\
\hline New York & 0 & 0 & 0 & 11 & 0 & 0 \\
\hline Oho & 1 & 1 & 10 & 36 & 25 & 752 \\
\hline 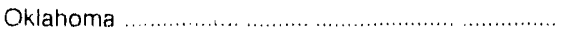 & 5 & 3 & 53 & 59 & 34 & 7,580 \\
\hline Pelinsylvania. & 0 & 0 & 0 & 36 & 32 & 731 \\
\hline Texas & 1 & 0 & 0 & 78 & 36 & 9,070 \\
\hline West Virgınia & 19 & 18 & 96 & 106 & 84 & 1,420 \\
\hline 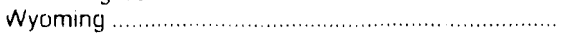 & 1 & 0 & 0 & 2 & 1 & 90 \\
\hline (1) & 33 & 22 & 159 & 792 & 591 & 66,255 \\
\hline
\end{tabular}

a New natural gas and certain natural gas produced from the Outer Continental Shelf.

- New onshore production wells.

c High cost natural jas.

d Stripper well natural gas.

e Not all filings report estimated volumes. The "With Volumes" columns show number reporting volumes.

- Annual volumes are often estimated by producers prior to actual operations or based on short periods of operation. For this reason the accuracy of estimates may vary.

Notes and Sources: See the last page of this section. 
Table 10. Well Determination Filings by Cat yoiry, September 1990 (Volumes in Million Cubic Feet)

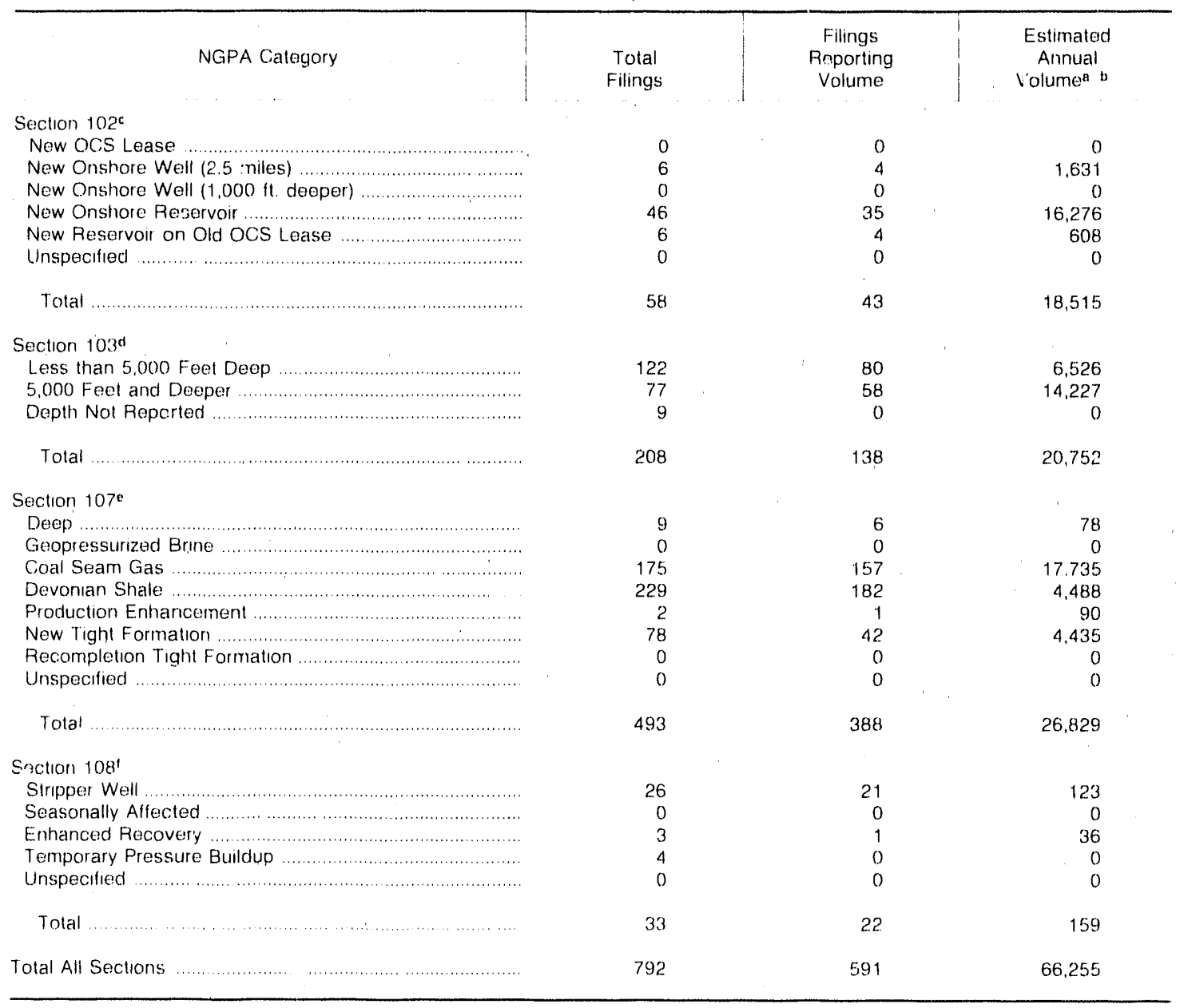

a Includes all fiungs reporting volume.

- Annua! volumes are often estimated by producers prior to actual operations or based on short periods of operation. For this reason the accuracy of estimates may vary.

c New natural gas and certein natural gas produced from the Outer Continental Shelf.

d New onshore prodiction wells.

- High cost natural gas.

1 Stripper well natural gas.

Notes and Sources: See the last page of this section. 
Table 11. Natural Gas Ceiling Prices by Category of Gas, Tyne of Sale, or Contract ${ }^{\mathrm{a}}$ (Dollars per Million Btu)

\begin{tabular}{|c|c|c|c|c|c|c|c|}
\hline NGPA Category & $\begin{array}{l}\text { Nov } \\
1990\end{array}$ & $\begin{array}{l}\text { Nov. } \\
1989\end{array}$ & $\begin{array}{l}\text { Nov. } \\
1988\end{array}$ & $\begin{array}{l}\text { Nov. } \\
1987\end{array}$ & $\begin{array}{l}\text { Nov. } \\
1986\end{array}$ & $\begin{array}{l}\text { Nov. } \\
1985\end{array}$ & $\begin{array}{l}\text { Nov. } \\
1984\end{array}$ \\
\hline \multicolumn{8}{|l|}{ Section 102} \\
\hline New Natural Gas, Certain OCS Gas" & 5.868 & 5.490 & 5.058 & 4.715 & 4.403 & 4.116 & 3.821 \\
\hline \multicolumn{8}{|l|}{ Section $103(\mathrm{~b})(1)$} \\
\hline 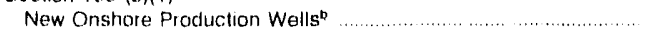 & 3.623 & 3.497 & 3.345 & 3.238 & 3.141 & 3.047 & 2.942 \\
\hline \multicolumn{8}{|l|}{ Section $103(b)(2)$} \\
\hline 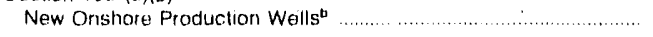 & -- & - & 3.345 & .000 & 3.772 & 3.582 & -- \\
\hline \multicolumn{8}{|l|}{ Section $105^{\circ}$} \\
\hline 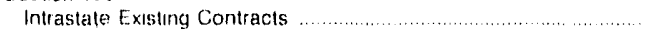 & 5.567 & 5.248 & 4.879 & 4.593 & 4.330 & 4.084 & $\cdots$ \\
\hline \multicolumn{8}{|l|}{ Section $106(b)(1)(B)$} \\
\hline \multirow{2}{*}{\multicolumn{8}{|c|}{ Alternate Maxımum Lawlul }} \\
\hline \multicolumn{7}{|l|}{ Price for Certain Intrastate } & \\
\hline Rollover Gas ${ }^{d}$ & 2.073 & 2.001 & 1.913 & 1.853 & 1.796 & 1.744 & 1.681 \\
\hline \multicolumn{8}{|l|}{ Section 107} \\
\hline 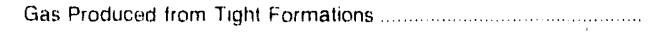 & 7.246 & 6.994 & 6.690 & 6.476 & 6.282 & 6.094 & 5.884 \\
\hline \multicolumn{8}{|l|}{ Section 108} \\
\hline Stripper Gas & 6.284 & 5.879 & 5.416 & 5.049 & 4.716 & 4.405 & 4.092 \\
\hline \multicolumn{8}{|l|}{ Section 109} \\
\hline Not Otherwise Covered ............... & 2.998 & 2.895 & 2.771 & 2.683 & 2.601 & 2.525 & 2.436 \\
\hline \multicolumn{8}{|l|}{ Section 104 and 106 (a) } \\
\hline 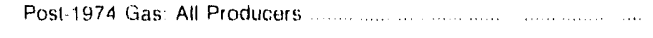 & 2.998 & 2.895 & 2.771 & 2.683 & 2.601 & 2.525 & 2.436 \\
\hline \multicolumn{8}{|l|}{ 1973.1974 Biennium Gas: } \\
\hline 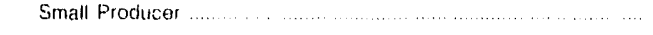 & 2.529 & 2.444 & 2.339 & 2.266 & 2.199 & 2.135 & 2.063 \\
\hline Large Producer ................. & 1.940 & 1.872 & 1.790 & 1.733 & 1.680 & 1.629 & 1.572 \\
\hline \multicolumn{8}{|l|}{ Inte. state Rollover Gas:e } \\
\hline Small Producer ............... & 1.112 & 1075 & 1.028 & .996 & .967 & .937 & .904 \\
\hline Large Producer & 1.112 & 1.075 & 1.028 & .996 & .967 & 937 & .904 \\
\hline \multicolumn{8}{|l|}{$\begin{array}{l}\text { Replacement Contract Gas or } \\
\text { Recompletion Gas }\end{array}$} \\
\hline Small Producer $\quad \ldots \ldots \ldots \ldots \ldots \ldots$ & 1.425 & 1.375 & 1.315 & 1.271 & 1.233 & 1.199 & 1.157 \\
\hline 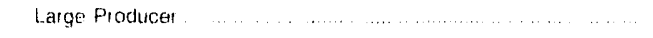 & 1.088 & 1.051 & $1.00 \%$ & 977 & .948 & 918 & .885 \\
\hline \multicolumn{8}{|l|}{ Flowing Gas: } \\
\hline Simall Producer $\ldots \ldots \ldots \ldots$ & .718 & 694 & .664 & .645 & 625 & .607 & .586 \\
\hline 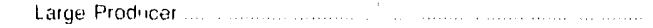 & .605 & 584 & 561 & .545 & .525 & .512 & .495 \\
\hline \multicolumn{8}{|l|}{ Certain Permian Basin Gas } \\
\hline Small Producer $\ldots$ & 847 & 819 & .784 & .756 & .735 & 714 & .690 \\
\hline Large Producer $\quad \ldots . . . . . . . \ldots \ldots$ & 750 & 723 & 691 & 671 & 651 & 633 & .609 \\
\hline \multicolumn{8}{|l|}{ Cerlann Rocky Mountan Gas: } \\
\hline Small Producer & 847 & 819 & .784 & .756 & .735 & .714 & 690 \\
\hline 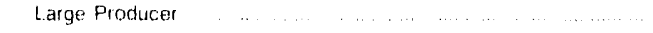 & 750 & 694 & 664 & 645 & 625 & 607 & .586 \\
\hline \multicolumn{8}{|l|}{ Certan Appalachan Basin Gas } \\
\hline North Subarea Contracts dated & & & & & & & \\
\hline after Cot $7.1969 \quad \ldots \ldots \ldots \ldots$ & 684 & 660 & 630 & 614 & .594 & .576 & .555 \\
\hline Othen Contracts $\ldots \ldots \ldots \ldots \ldots$ & 634 & 611 & .585 & .569 & 549 & .531 & .513 \\
\hline $\begin{array}{l}\text { Minimurn Rate Gas All Producers } \\
\text { (clollars per Mcl }\end{array}$ & & & & & & & \\
\hline fdollars per Mcli & 372 & 359 & 347 & .335 & 326 & 314 & .302 \\
\hline
\end{tabular}

- Se: Appendix A. Explanatory Nole 10 for discussion of Title I of the Natural Gas Policy Act

b Commencing January 1, 1985 and July 1.198\%, the price of some natural gas finally determined to be natural gas produced trom a new. onshore production well unde, Section 103 is deregulated Thus, for all months succeeding dure 1987 publication of a maximum lawful price per MMBtu under NGPA Section 103(b)(2) is dis com."lued

c New calecgory as of January 1,1985

"Section $106($ th) of the NGPA provides that lor certain gas sold under an intrastate rollover contract, the maximum lawful price is the higher of the price paid under the expured contract adjusted for inflation of an alterrialive Maximum l.awful Price sipecitied in this table This alternative Maximum Lawful Price for each monith appe:ars in this row

- The: price for interstate roliover gas is the higher of the price listed in this lable or the fust and leasonatle price under the expired contract as adjusted for inflation

Notes and Sources See the last page of this section 

Table 12. Revenues, Expenses, and Income of Major Interstate Natural Gas
Pipeline Companies (Million Dollars)

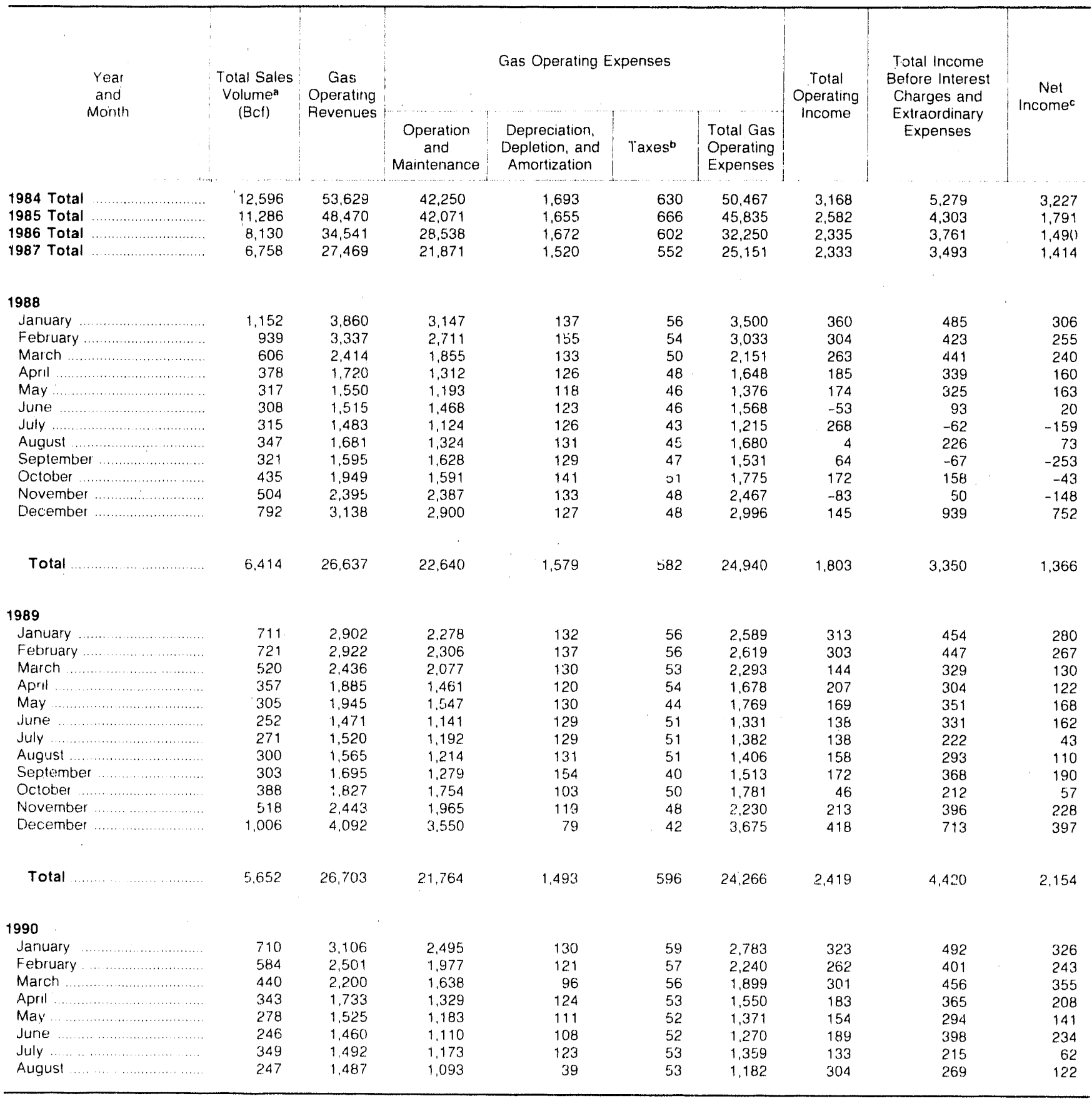

a Inctudes sales for resale and sales to ultimate consumers.

- Excludes income taxes.

c Total Income before Interest Charges and Extraordinary Expenses and Investment Tax Credits minus income Taxes, Interest Charges, and Extraordi. nary ltems

Notes and Sources: See the last page of this section. 
Table 13. Volumes and Prices of Natural Gas Sold by Major Interstate Natural Gas Pipeline Companies (Volumes in Million Cubic Feet, Prices in Dollars per Thousand Cubic Feet)

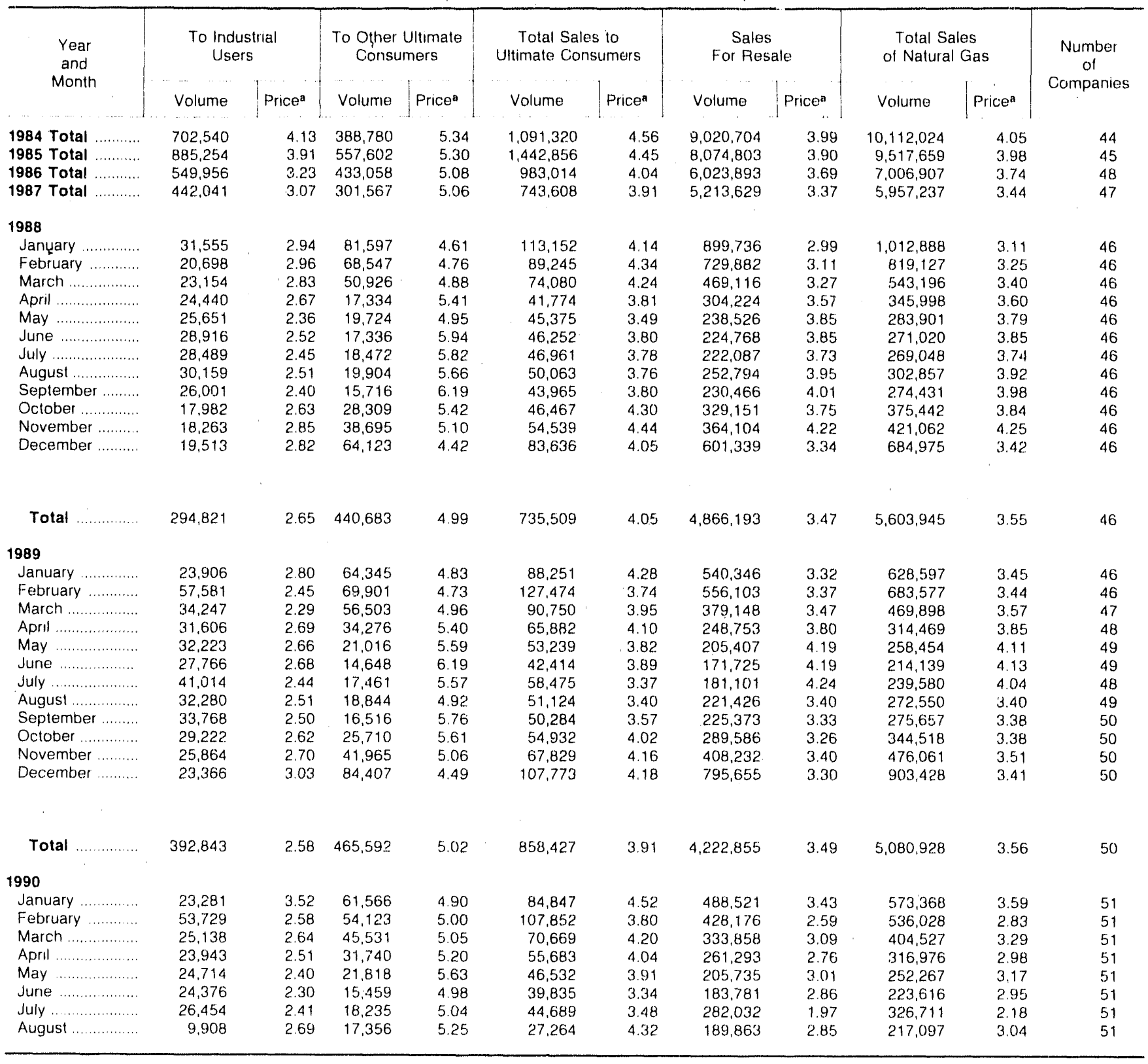

a All prices are weighted averages.

Notes and Sources: See the last page of this section. 
Table 14. Volumes and Prices of Natural Gas Sold by Major Interstate Natural Gas Pipeline Companies, by Company, August 1990 (Volumes in Million Cubic Feet, Prices in Dollars per Thousand Cubic Feet)

\begin{tabular}{|c|c|c|c|c|c|c|c|c|c|c|}
\hline \multirow{2}{*}{ Piperirm Company } & \multicolumn{2}{|c|}{$\begin{array}{l}10 \text { Industrial } \\
\text { Users }\end{array}$} & \multicolumn{2}{|c|}{$\begin{array}{l}\text { To Other Ultimale } \\
\text { Consumers }\end{array}$} & \multicolumn{2}{|c|}{$\begin{array}{l}\text { Tolal Saless to } \\
\text { Unturnate Corsumers }\end{array}$} & \multicolumn{2}{|c|}{$\begin{array}{c}\text { Sales } \\
\text { for Rasale }\end{array}$} & \multicolumn{2}{|c|}{$\begin{array}{l}\text { Total Sales } \\
\text { of Natural Gass }\end{array}$} \\
\hline & Volume & Pricen & Volume & Pricen & Volume & Pencen & Volume & Price" & Volume & Price" \\
\hline Algonguning Gits & 0 & 000 & (1) & 000 & 0 & $0(0)$ & $42 \% 4$ & $5+58$ & $4.2 \% 4$ & $5,6 \mathrm{H}$ \\
\hline AP.JA & 0 & 00 & i) & 00 & 0 & 00 & 4.947 & 213 & 4,947 & 273 \\
\hline Aitla inc: & 3051 & 269 & $7.42 \mathrm{H}$ & 682 & 10,479 & $s t 5 \%$ & 273 & 148 & $10.75 ?$ & 5,52 \\
\hline Hoar crotek Skrticke & 0 & 00 & 0 & 00 & 0 & (o) & 0 & 00 & () & 00 \\
\hline Canyon Crett. Mansm co & 0 & 00 & 0 & 00 & 0 & 00 & 0 & o() & 0 & 00 \\
\hline Colorado Intristars & 96 & $2 A B$ & 0 & 00 & 46 & 248 & 3019 & $245_{1}$ & 3,116 & 245 \\
\hline Columblat Gas, Iantsm & 0 & 00 & ! & 00 & 0 & $(0)$ & 3,416 & 3.15 & 3.916 & 315 \\
\hline Cooluentba coull & 0 & 00 & 3 & 300 & 3 & 300 & 0 & 00 & 3 & 300 \\
\hline Consolledated Gads & 5,5 & 231 & 0 & 00 & 5,5 & 231 & 1,381 & 150 & 1.436 & 153 \\
\hline East Tentessene: & $1600^{2}$ & $25 \%$ & 1 & 400 & 1.603 & 25,5 & 3,239 & 241 & 4,442 & ?.45 \\
\hline Ellatso & $19 ? 1$ & 194 & 0 & 00 & 1.921 & 194 & 3.121 & 226 & $5,04 ?$ & 214 \\
\hline I quitrans. & 0 & 00 & 0 & 00 & 0 & 00 & 1,524 & 439 & 1.525 & 139 \\
\hline Foneda Gats & $2.23 \%$ & 313 & 0 & 00 & 2,232 & 313 & 4,608 & 246 & (j.920 & 267 \\
\hline Greal l akes Cial. & 0 & 00 & 0 & 00 & 0 & 00 & 1,199 & 384 & 1.199 & 384 \\
\hline High lsland Offstrore: & 0 & 00 & 0 & 00 & 0 & 00 & 0 & 00 & 0 & 00 \\
\hline h. N Energy inc. & 39 & 304 & 3,703 & $3.9 \%$ & 3,742 & 391 & 75, & 330 & 4,497 & 381 \\
\hline Michuyan Consolidaled & 265 & 473 & 5,340 & 453 & 5,605 & 454 & 1 & 200 & 5,606 & 454 \\
\hline Michigan Gas Storaqe & 0 & 00 & 0 & 00 & 0 & 00 & 754 & 364 & 754 & 364 \\
\hline Midwestern & 0 & 00 & i) & 00 & 0 & 00 & 3886 & 271 & 3,886 & 271 \\
\hline Mississippt Hiver & 1.44 & $346 i$ & c & 00 & las & 346 & 1.713 & 629 & $1,86 ?$ & 606 \\
\hline Mounilater Fuel Rers & 0 & 0,0 & 0 & 00 & 0 & 00 & 1.786 & 512 & 1,788 & t).12 \\
\hline Pvational f wes & 0 & $O 0$ & 0 & 00 & 0 & 00 & 3.727 & 393 & 3.727 & 393 \\
\hline Natural Gas Pipetires & 2 & 250 & D & 00 & $?$ & 250 & $15,22 ?$ & 4.32 & 15.224 & 4.32 \\
\hline Pvotheress Bordes & 0 & 00 & 0 & 00 & () & 00 & 0 & 100 & 0 & 00 \\
\hline Norlmerp Hatural & () & 00 & 1 & 1 (in) & $i$ & 100 & 20.844 & 241 & 20.885 & 211 \\
\hline Nrontrwest Alaskan & 0 & 00 & 0 & 00 & 0 & 00 & 32329 & 159 & 32320 & 159 \\
\hline 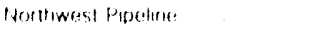 & $?$ & 150 & ) & 0,1 & ? & 150 & 1,314 & 3.91 & 1,316 & $3 \div 0$ \\
\hline Overthrust piptelne? & 0 & 00 & (3) & 00 & 0 & $0(0)$ & 0 & 00 & 0 & 00 \\
\hline Patalic Gad Transm & 0 & 00 & 0 & 00 & 0 & or & 28,684 & 241 & $2 B, 6, B A 4$ & 241 \\
\hline Facithe, Irilerstale & 0 & 00 & 0 & 00 & 0 & 00 & 6,889 & 53 & 6,881 & 53 \\
\hline Wanhande fastem & 32 & $\because 00$ & $80 t$ & 195 & 837 & $19 t$ & 5.703 & 242 & 6,540 & 236 \\
\hline Satmer: Pipeline Cos & () & 00 & ) & 00 & 1) & (x) & 0 & 00 & 0 & 00 \\
\hline Sera a Fobsir Pigetine. & 0 & 00 & 0 & 00 & 0 & $0 n$ & 0 & 00 & 0 & 00 \\
\hline Sisutherm Natural & 21 & 376 & 0) & $x$ & 21 & 376 & 4,2834 & 247 & 4.306 & $? 48$ \\
\hline Shingray fipetirese & 0 & of & 0 & 00 & 0 & 00 & 0 & 00 & 0 & 00 \\
\hline Tenneces lex & $11 \%$ & $? 69$ & 0 & jo & 112 & 269 & 3771 & 340 & 3.883 & $33 B$ \\
\hline Texas ! ablem & 0 & 00 & () & 00 & 0 & $(0)$ & 15,677 & $43 t$ & 15,677 & 435 \\
\hline Tuxas Gas Parism & 0 & 0 & 0 & 00 & ()) & (c) & 7,033 & 397 & 7.033 & 397 \\
\hline 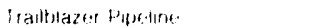 & 0 & 001 & 0 & 00 & () & 00 & 0 & 100 & 0 & 00 \\
\hline Transco Gas & 0 & (6) & 0 & (o) & o) & $(0)$ & 0 & 00 & () & 00 \\
\hline Transconterental & $1 H$ & 250 & 0 & or & $1 H$ & 250 & 21.052 & 258 & 21,070 & 258 \\
\hline Tranlswestern Fipotine & "4 & 178 & 21 & 190 & 30 & $1 \mathrm{H \%}$ & 30038 & 130 & 3.068 & 130 \\
\hline Tturikline Gas Cog & 1 & 200 & 35 & $? 14$ & 36 & 214 & 8,041 & 375 & 8,077 & $3 \% 4$ \\
\hline Trurikline I $\mathrm{Nr}_{3}$ & ) & 00 & 0 & 00 & 0 & 00 & (1) & 00 & 0 & 00 \\
\hline U. 1 ollshort & 0 & 60 & 0 & 00 & 0 & 00 & 0 & 00 & 0 & 00 \\
\hline Urilled Gas, Pipeline: & 173 & 313 & 17 & 276 & 190 & 3113 & 670 & 391 & 860 & 529 \\
\hline Valeto Interblale: Tranism & 0 & 00 & (1) & 190 & 0 & 00 & 0 & 00 & 0 & ors \\
\hline Viburg Gas combany & 0 & $(0)$ & () & (o) & () & $0 \theta$ & 240 & 173 & 240 & 173 \\
\hline Wiltams Waturat & $12 \beta 3$ & 243 & 2 & 200 & 130 & $24 \%$ & 811 & $43 i j$ & 947 & 415 \\
\hline Willistor fiasint & 0 & 00 & 0) & 00 & 0 & orj & 5,7 & 389 & 57 & 389 \\
\hline Wyoming lenterstale & ) & 00 & 0 & (1) & ) & 00 & () & ou & 0 & 00 \\
\hline Trital Avertays Perese & 4905 & 269 & 1735 & 520 & 27.264 & $43 \%$ & $219,85,3$ & $28{ }^{2}$ & 247.117 & 301 \\
\hline \multicolumn{11}{|l|}{ Salos lo (itiver Major } \\
\hline Compatruess & & & & & & & 29.940 & $2 \mathrm{HA}$ & 29990 & 2. 34 \\
\hline \multirow{2}{*}{\multicolumn{11}{|c|}{ Sales Excluding Sales 10}} \\
\hline & $\ldots$ & .. & . & -. & & & 189.863 & $28 r_{3}$ & 217.127 & 304 \\
\hline
\end{tabular}

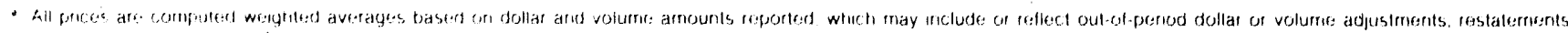

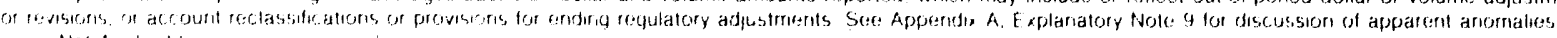
toot Abplicatele

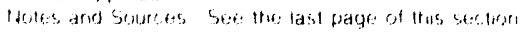


Table 15. Natural and Other Gases Produced and Purchased by Major Interstate Natural Gas Pipeline Companies

(Million Cubic Feet)

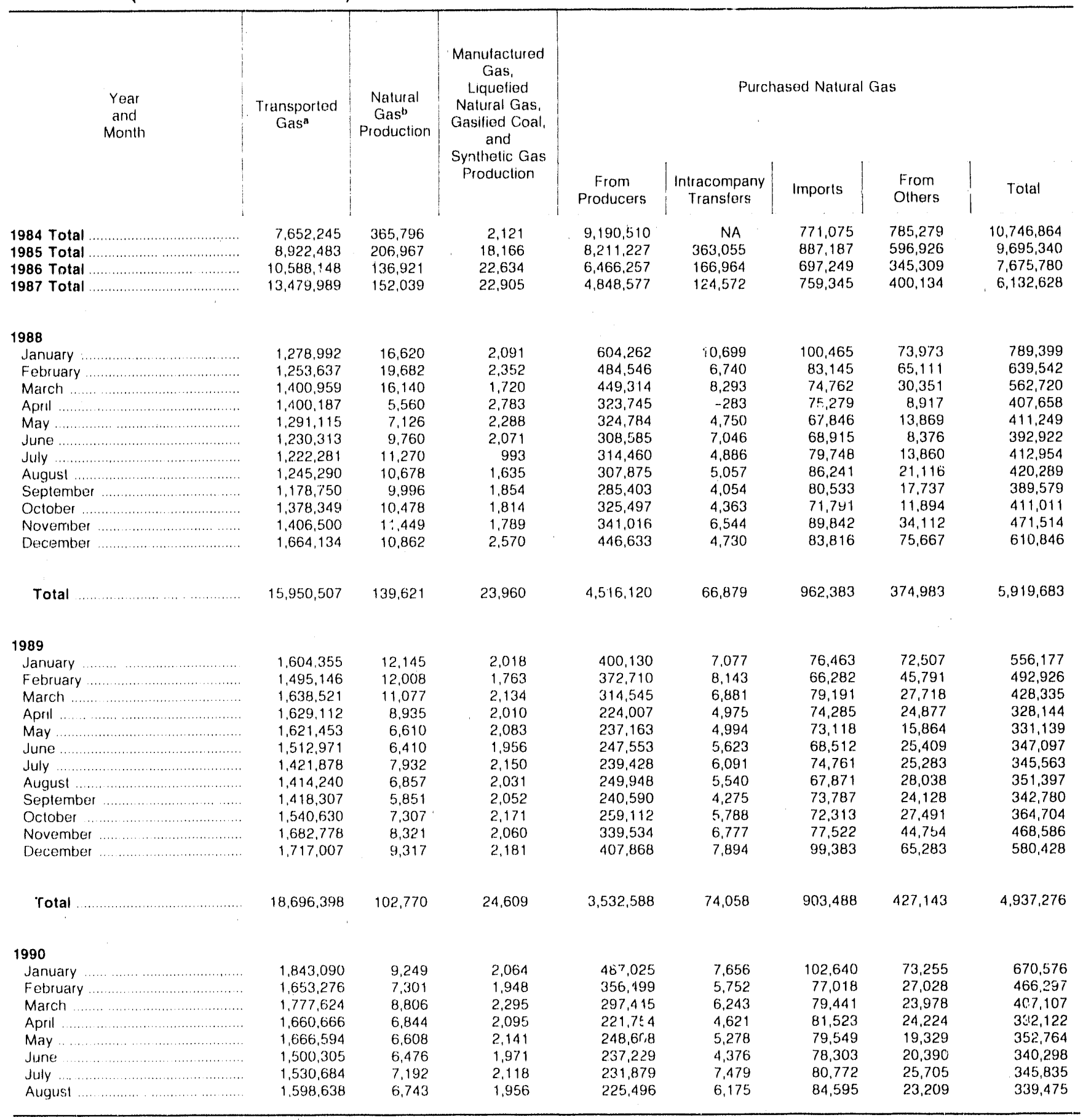

a Gas transported for other companies through the production, transmission, or distribution lines or compressor stations of the reporting pipelines

b Mixture of hydrocarbons existing in gaseous phase or in solution with crude oll in nalural underground reservoirs.

: Includes out-cif-period adjustments to correct data in prior month.

NA Not Ay. He.

Notes and So, . See the last page of this section 
Table 16. Natural and Other Gases Produced and Purchased by Major Interstate Natural Gas Pipeline Companies, August 1990

(Million Cubic Feet)

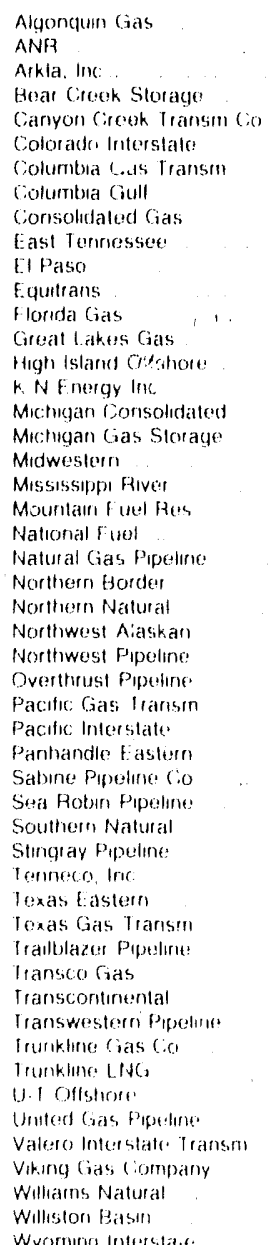

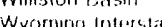

Teral

Purchases ferm Other Major

Companmes

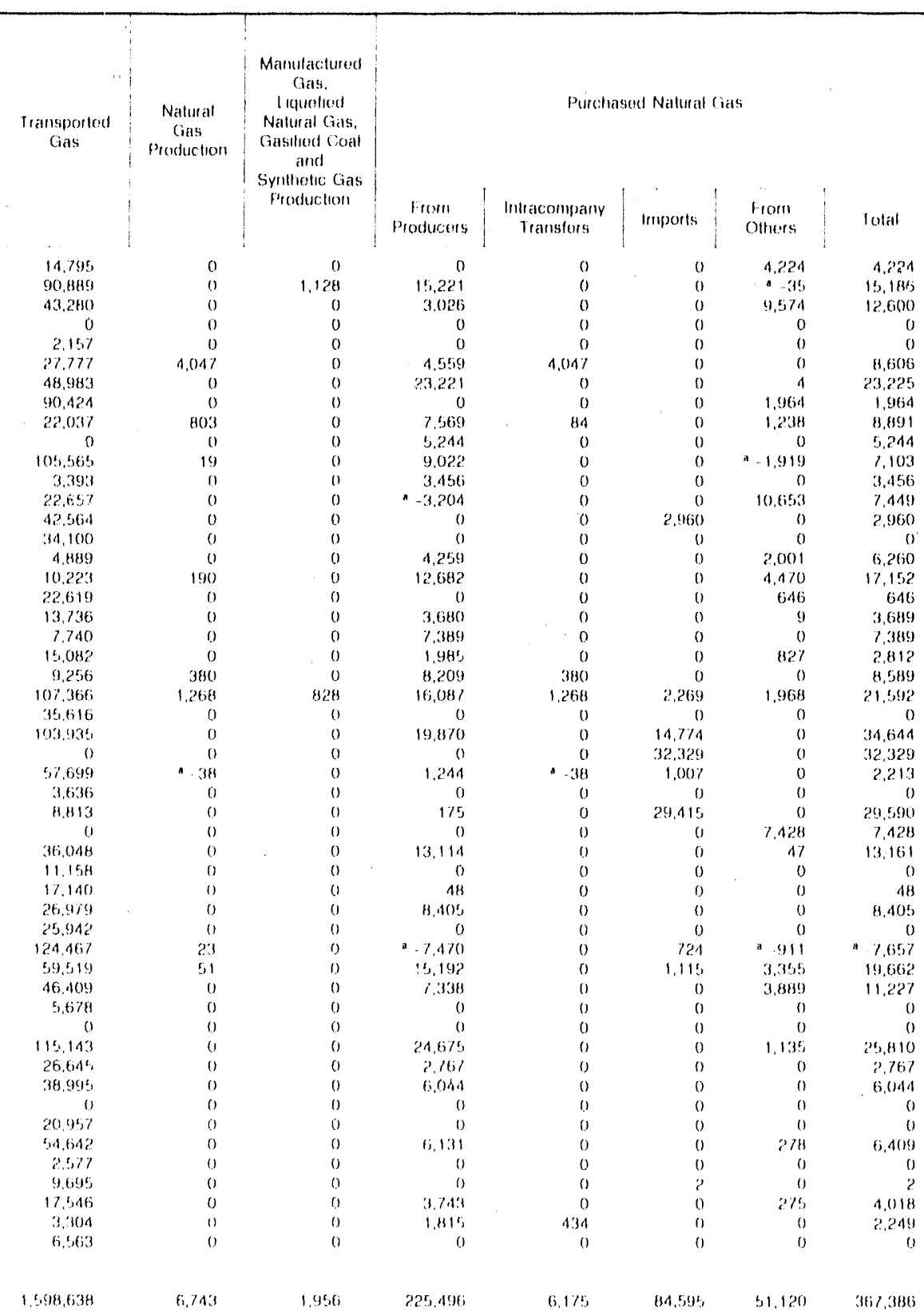

0

$\because 1,911$

27.911

Purchason, Excludenty

Puachesses: leom cother

Major Companme,

22,496

6,175

B4,, 095

23.200

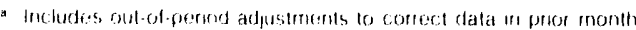
Not Appolicatise.

Woles and Sourcess See the last page ol thes section 
Table 17. Underground Natural Gas Storage

(All Operators)

(Volumes in Billion Cubic Feet)

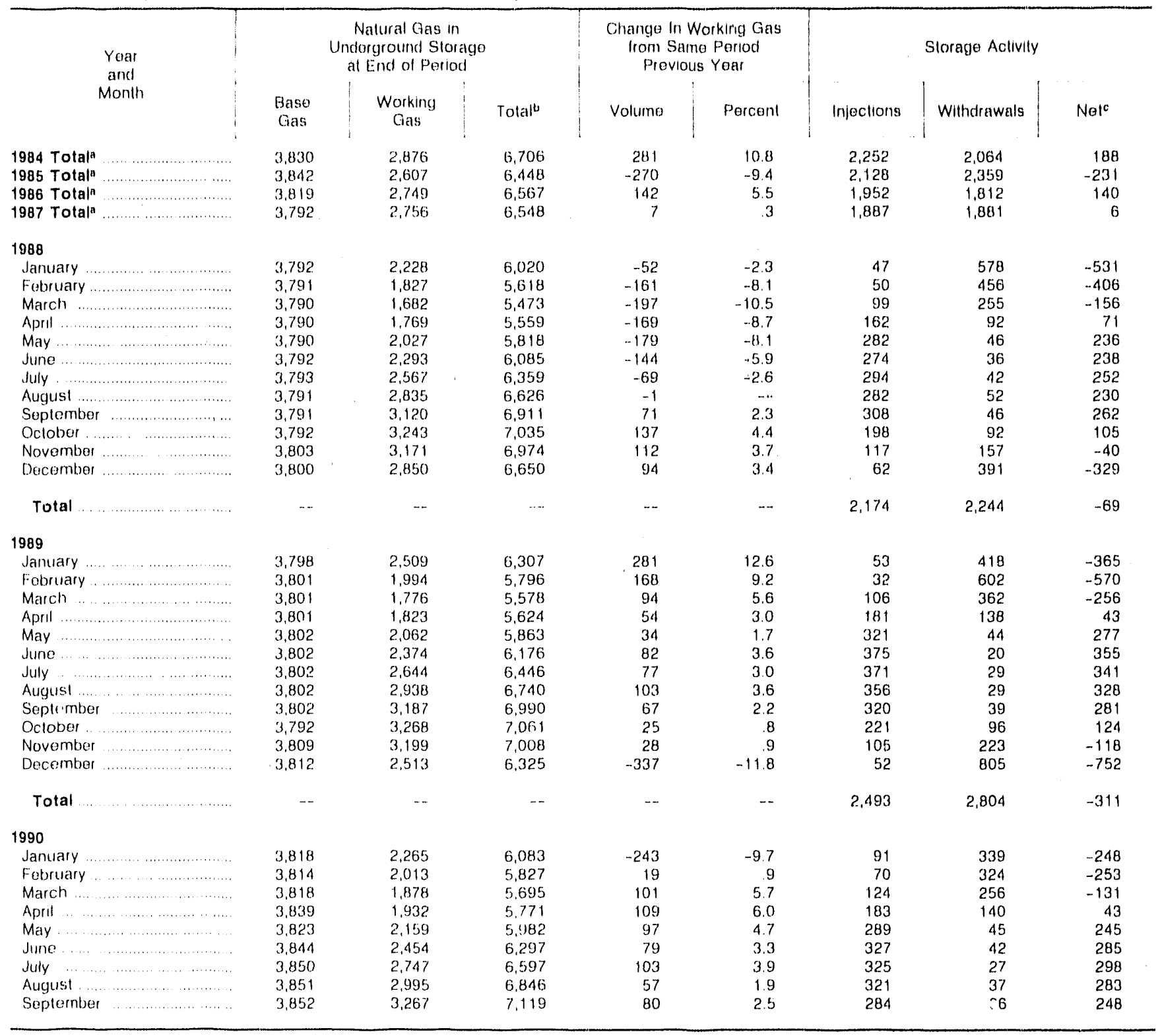

a Total as of December 31.

b Total underground storage capacity at the end of each calendar Year (in billion cubic leet): 1984 8,$043 ; 1985 \cdot 8,087 ; 1986 \cdot 8,145 ; 1987$ and 1988 . 8,124 , and $1989 \cdot 8,124$. Current total caparily is 8,125

c Positive numbers indicale the volume of injections in excess of withdrawals. Negative numbers indicate the volume of withdrawals in excess of injections

Notes and Sources: Seo the last page of this section 
Figure 6. Underground Natural Gas Storage in the United States

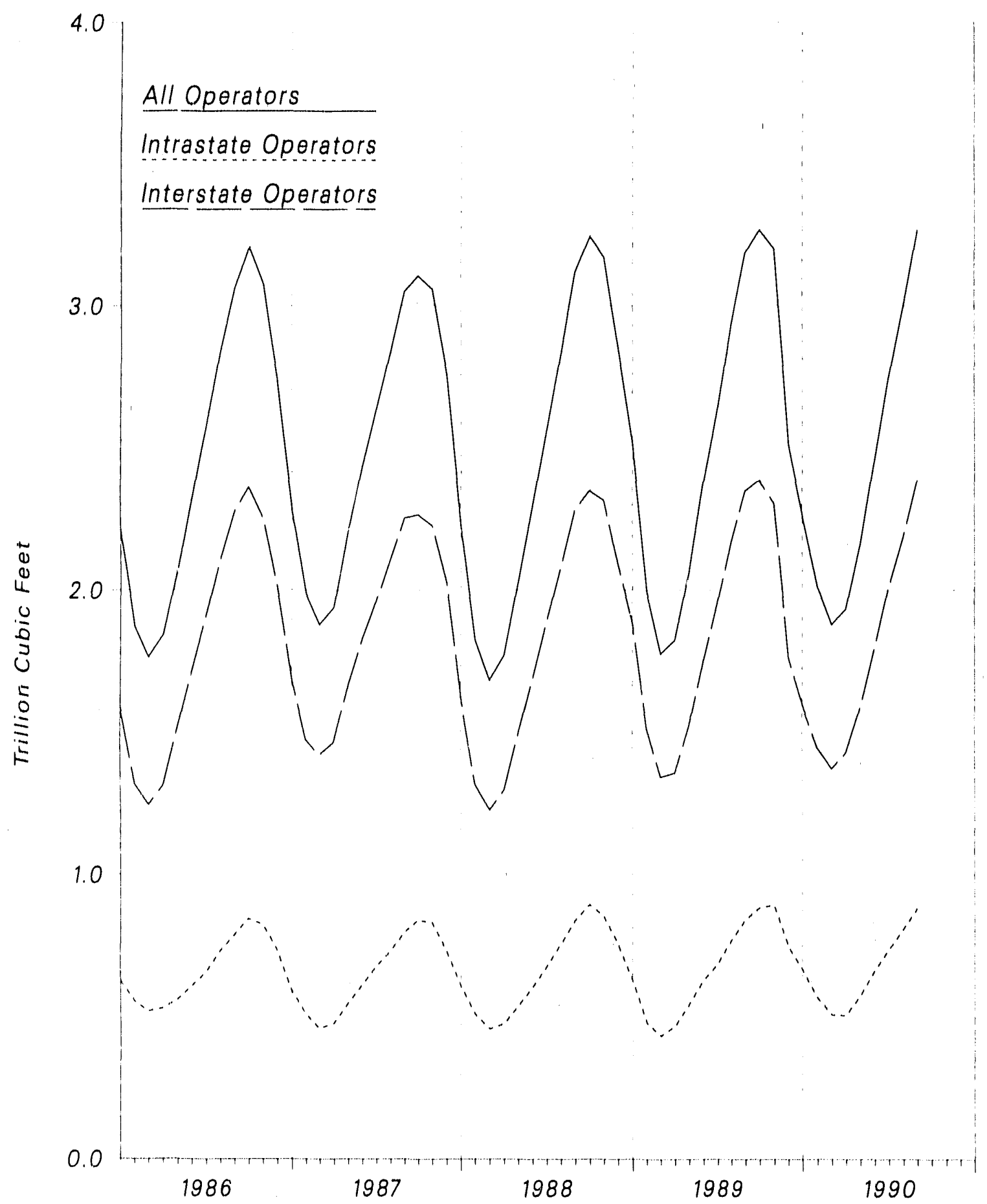

Source: Form EIA-191, Form FERC-8, and Form EIA-176. 
Table 18. Underground Natural Gas Storage (Interstate Operators of Storage Fields) (Volumes in Billion Cubic Feet)

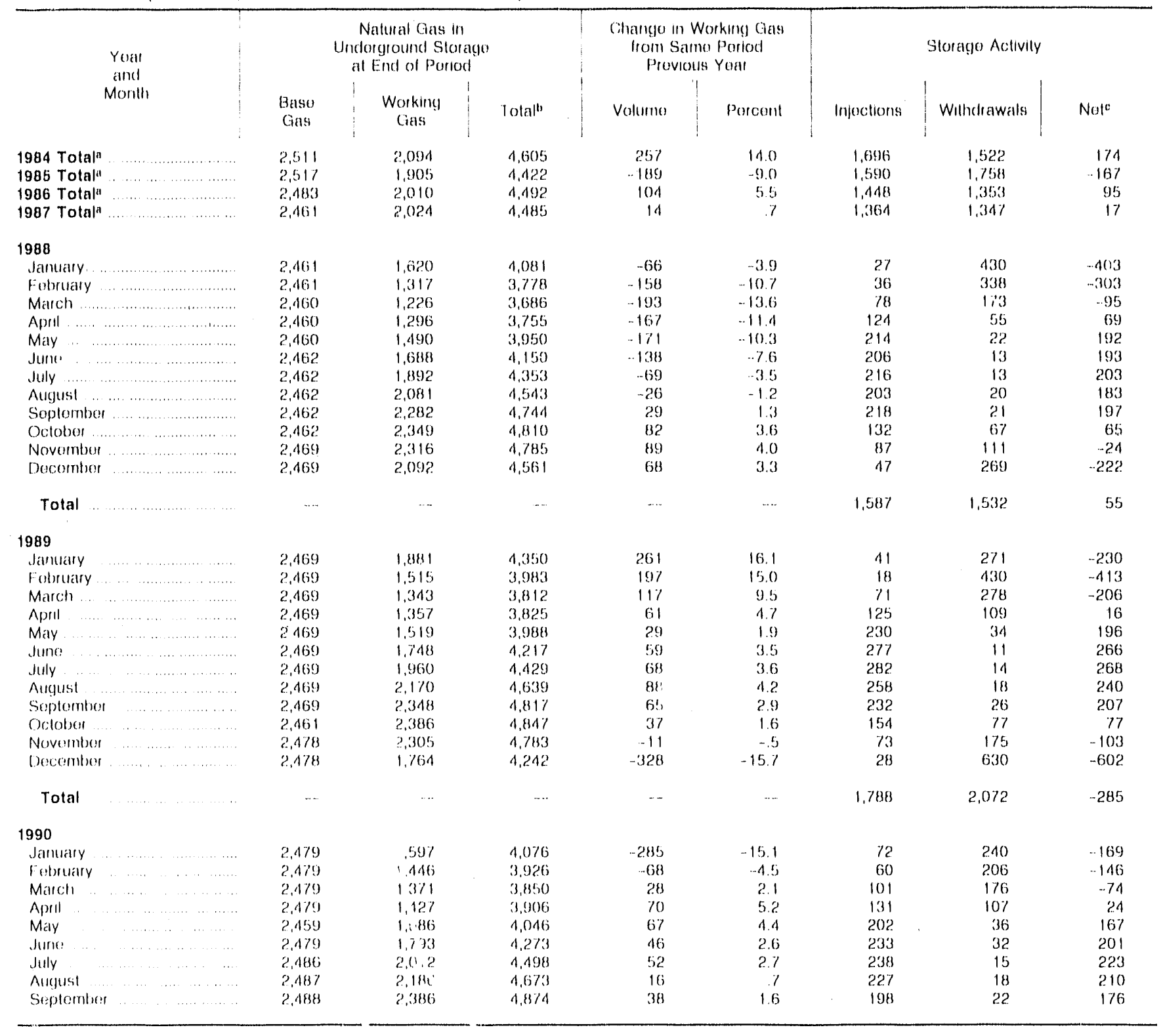

i) Total as of leocember 31

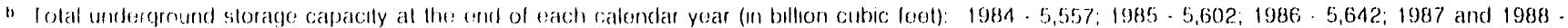

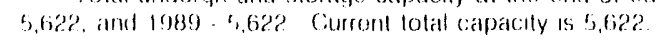

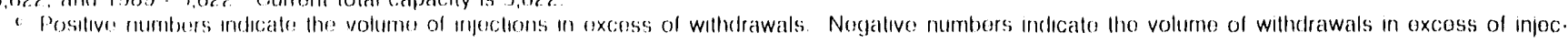
torisi

Nol Applicatile:

Notes and Sourcos: Soo llow last patye of thes socuon 
Table 19. Underground Natural Gas Storage (Intrastate Operators and Independent Producers)

(Volumes in Billion Cubic Feet)

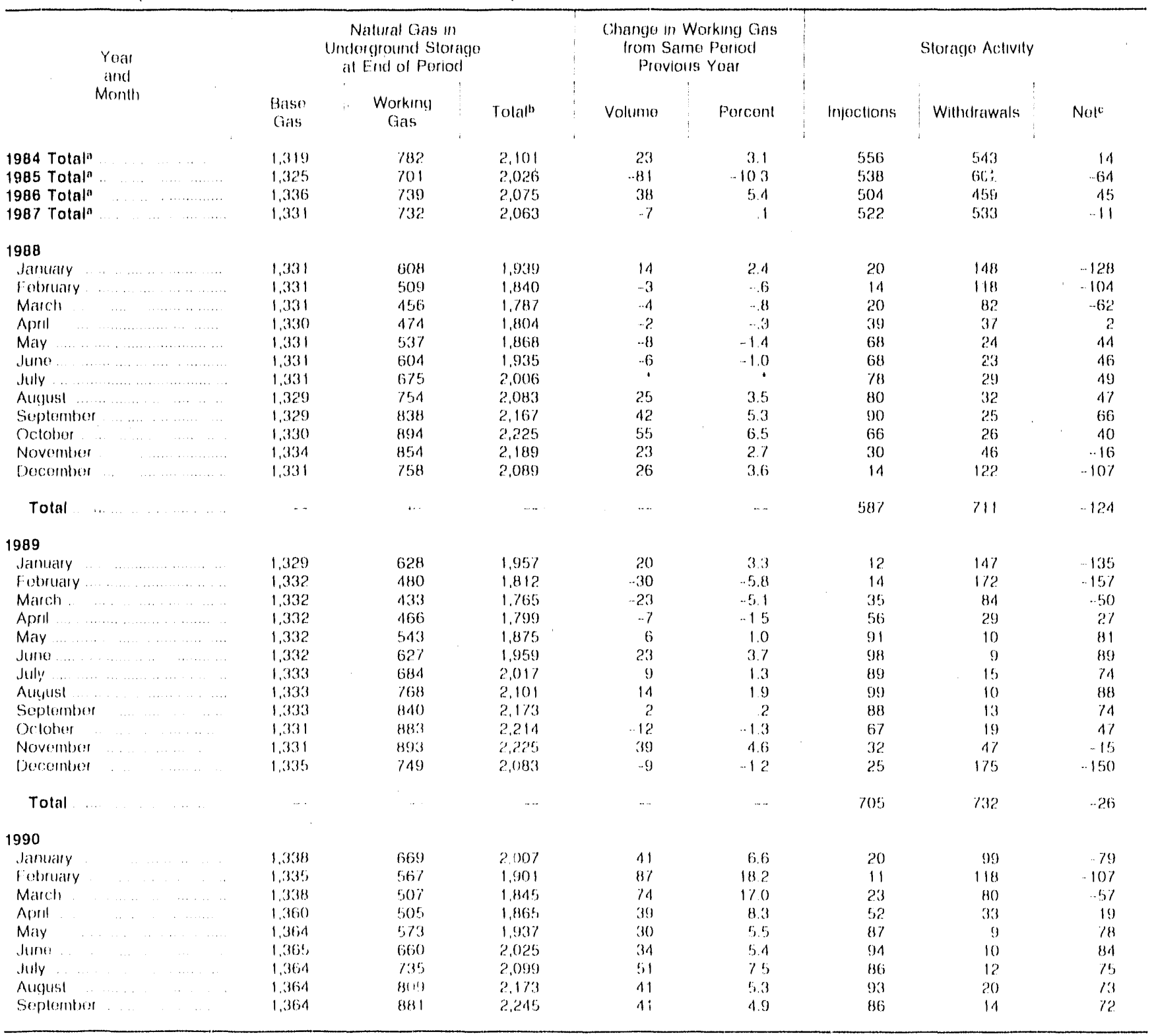

T Tolal as of Decomber 31

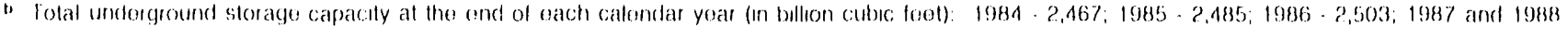
$2,8,5 \%$, and 19835 . 2,502 Current lotal lapactly is $2,503$.

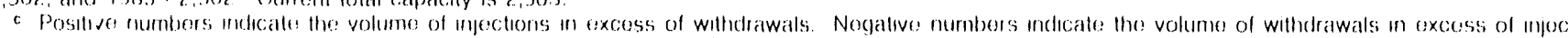
lioris

Nol Applicables.

Volume ts lessis than 600 mollion cubsic leot.

Noles and Sources. Sent the tasil patge of thes secton. 


\section{Table 20. Activities of Multi-State Underground Nativial Gas Storage Operators, September 1990}

(Volume in Million Cubic Feet)

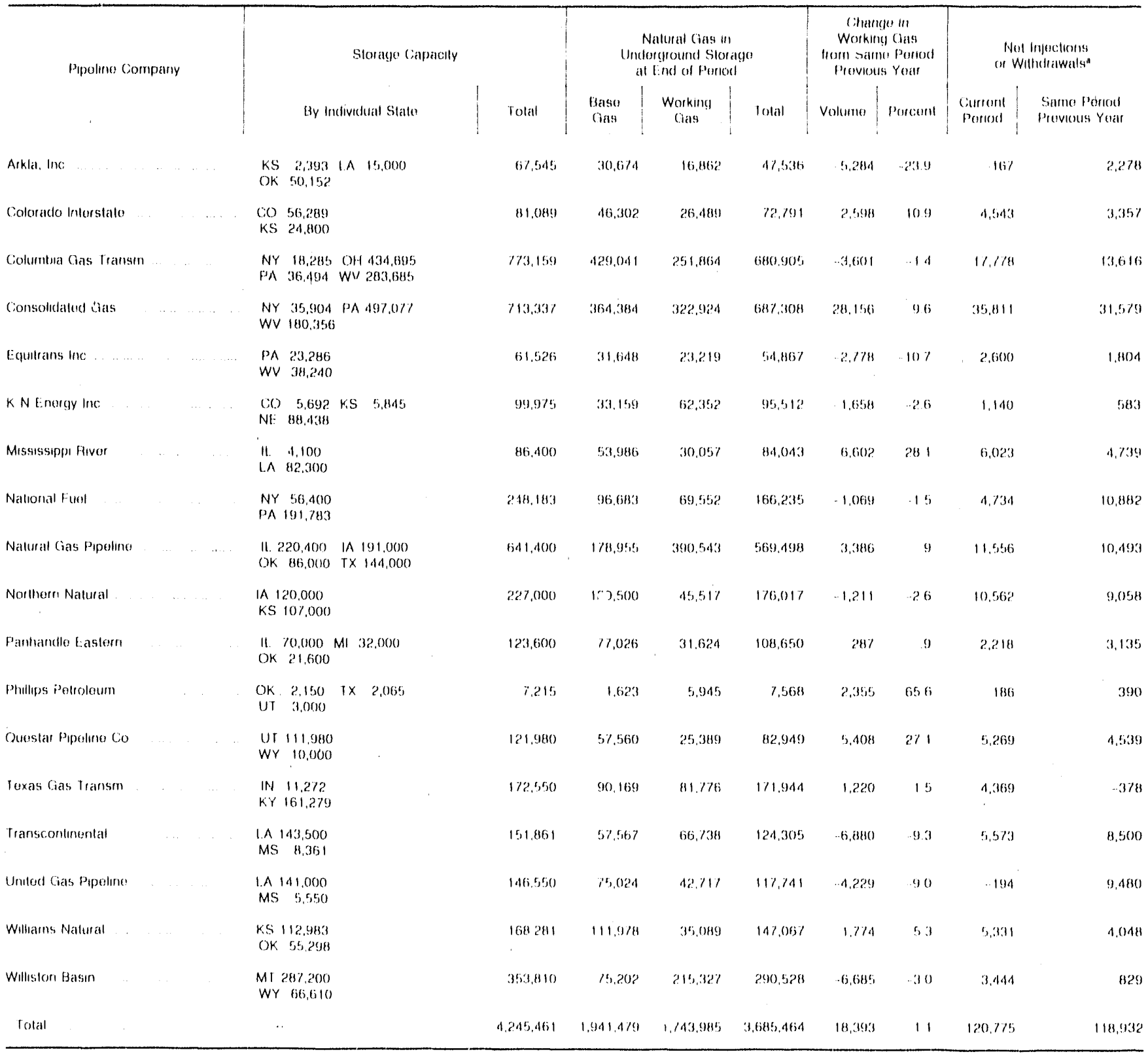

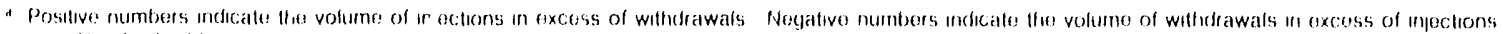
Nol Applitatsles

Notes and Sourcess seo the last pagen of It s soctoon 
Table 21. Activities of Single-State Underground Natural Gas Storage Operators, by State, September 1990

(Volumes in Million Cubic Feet)

\begin{tabular}{|c|c|c|c|c|c|c|c|c|}
\hline \multirow[t]{2}{*}{ Slato } & \multirow{2}{*}{$\begin{array}{l}\text { Tolal } \\
\text { Storage } \\
\text { Capacity }\end{array}$} & \multicolumn{3}{|c|}{$\begin{array}{l}\text { Nalural Gas in } \\
\text { Underground Slorago } \\
\text { al End of Period }\end{array}$} & \multicolumn{2}{|c|}{$\begin{array}{c}\text { Chango in Working Gas } \\
\text { Irom Samo Poriod } \\
\text { Provious Yoar }\end{array}$} & \multicolumn{2}{|c|}{$\begin{array}{l}\text { Not Injections } \\
\text { or Withdrawals" }\end{array}$} \\
\hline & & $\begin{array}{l}\text { Base } \\
\text { Gas }\end{array}$ & $\begin{array}{c}\text { Working } \\
\text { Gas }\end{array}$ & Tolal & Volume & Fercont & $\begin{array}{l}\text { Cuiront } \\
\text { Poriod }\end{array}$ & $\begin{array}{l}\text { Samo Period } \\
\text { Provious Yalar }\end{array}$ \\
\hline 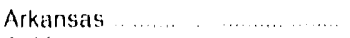 & 31.447 & 19,202 & 10,097 & 29,200 & 98 & 1.0 & 270 & 56 \\
\hline 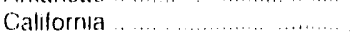 & 466,819 & 243,944 & 183,772 & 427,716 & 13,390 & 7.9 & 4.809 & 1,980 \\
\hline 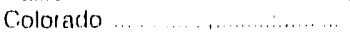 & 20,682 & 6,248 & 12,980 & 19,228 & 3,219 & 33.0 & 1,358 & 386 \\
\hline Illinols & 657,780 & 418,541 & 213,583 & 632,127 & 0.773 & 4.8 & 28,253 & $26,8 \% 1$ \\
\hline 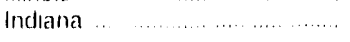 & 100,774 & 68,941 & 26.111 & 95,052 & 1,081 & 4.3 & 3,399 & 3,007 \\
\hline Kansas. & 81,905 & 37,390 & 28,058 & 65.449 & 824 & 3.0 & 1,311 & 1,389 \\
\hline 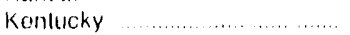 & 45,325 & 21,351 & 17.458 & 38,809 & -702 & -3.9 & 3,231 & 3,653 \\
\hline Loulsiana & 177.219 & 79,751 & 54,554 & 134,304 & 333 & .6 & 5,202 & 2,151 \\
\hline 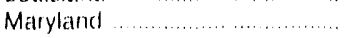 & 61.978 & 46,677 & 13.178 & 59,856 & 2,724 & 26.1 & 2,005 & $-1,527$ \\
\hline 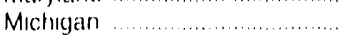 & 950,362 & 380,729 & 503,126 & 883,855 & 51,354 & 11.4 & 47,873 & 53,476 \\
\hline 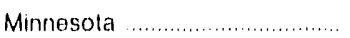 & 7,000 & 4,655 & 2.291 & 6,946 & $7 d$ & 3.3 & 56 & 0 \\
\hline Mississippi & 94,297 & 41,138 & 18,978 & 90,116 & 539 & 1.1 & 2,807 & 3,778 \\
\hline 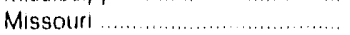 & 29,791 & 21,600 & 7.898 & 29,498 & 103 & 1.3 & 503 & 292 \\
\hline 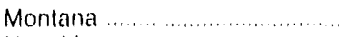 & 86,760 & 45,800 & 24,700 & 70,500 & 131 & .5 & 921 & 737 \\
\hline 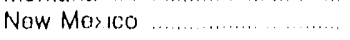 & 94,600 & 20.204 & 21,817 & 42,021 & 2,686 & 14.0 & 1,553 & 686 \\
\hline Now York & 45,670 & 19,995 & 21.443 & 41.437 & 1,101 & 5.4 & 2,972 & 2,272 \\
\hline Ohio $\ldots \ldots \ldots \ldots \ldots$ & 177,852 & 115,818 & 44,578 & 160,396 & 178 & 4 & 8,908 & 8,483 \\
\hline Oklahoma & 149,688 & 93,089 & 48,286 & 141,376 & $-3,502$ & -6.8 & 4,503 & 8,224 \\
\hline 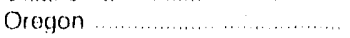 & 9,791 & 3.291 & 7.423 & 10.714 & 2,606 & 54.1 & 372 & 338 \\
\hline Ponnsylvania & 56,754 & 25,638 & 14,037 & 39,676 & 629 & 4.7 & 2.140 & 1,369 \\
\hline 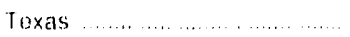 & 443,716 & 151,049 & 190,830 & 341,879 & $-21,072$ & -9.9 & -215 & 9,361 \\
\hline Washıngton & 36,400 & 21,300 & 11,839 & 33,139 & 41 & .3 & 978 & 2,094 \\
\hline Wost Vuginia & 20,851 & 9,793 & 9,385 & 19,178 & -512 & -5.2 & 1,000 & 1,066 \\
\hline 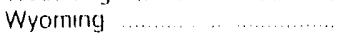 & 27.221 & 13,990 & 6,916 & 20,906 & 566 & 8.9 & 449 & -40 \\
\hline 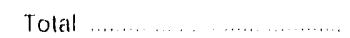 & $3,874,6831$ & $1,910,137$ & $1,523,338$ & $3,433,476$ & 65,663 & 4.5 & 127,668 & 130,106 \\
\hline
\end{tabular}

" Positive numbers indicate the volume of injections in excess of withdrawals. Negativo numbers indicate the volume of withdrawals in excess of injec trons

Notes and Sources See the last page of this section. 
Table 22. Natural Gas Deliveries to Residential Consumers by State (Million Cubic Feet)

\begin{tabular}{|c|c|c|c|c|c|c|}
\hline State & $\begin{array}{l}Y T D \\
1990\end{array}$ & $\begin{array}{l}\text { YTD } \\
1989\end{array}$ & $\begin{array}{l}\text { YTD } \\
1988\end{array}$ & $\begin{array}{c}\text { August } \\
1990\end{array}$ & $\begin{array}{c}\text { July } \\
1990\end{array}$ & $\begin{array}{l}\text { June } \\
1990\end{array}$ \\
\hline 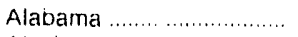 & 34,954 & 33,835 & 36,798 & 1.354 & 1.432 & 1.683 \\
\hline 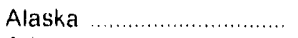 & 8,276 & 9,028 & 8,037 & 407 & 409 & 505 \\
\hline 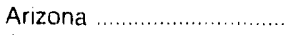 & 21,581 & 19,580 & 20,982 & 803 & 873 & 1.119 \\
\hline Arkansas ............................... & 28,798 & 30,166 & 31,855 & 1.110 & 1,154 & 1,384 \\
\hline 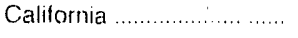 & 351,972 & 358,835 & 350,274 & 22,338 & ค 22,558 & A 25,181 \\
\hline 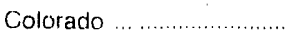 & 68,932 & 67,174 & 68,817 & 2,357 & 2,478 & 4,250 \\
\hline Connecticut ......................... & 26,026 & 27.351 & 27,188 & 934 & 1,104 & 1.310 \\
\hline 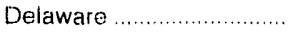 & 5,538 & 5,545 & 5.566 & 166 & 183 & 260 \\
\hline District of Columbia ......... & 11.676 & 12,548 & 12,618 & 445 & 505 & 614 \\
\hline Florida $\ldots . . . \ldots \ldots \ldots \ldots \ldots \ldots \ldots \ldots \ldots \ldots \ldots$ & 10,509 & 9,010 & 11,139 & 605 & 661 & 796 \\
\hline 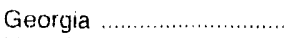 & 59,111 & 61,763 & 72,757 & 2,882 & 2,895 & 3,268 \\
\hline 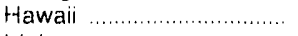 & 390 & 387 & 385 & 40 & 49 & 49 \\
\hline 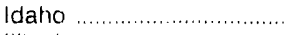 & 5.058 & 6,224 & 5,395 & 160 & 173 & 314 \\
\hline 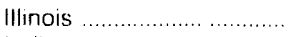 & 250.6 & 312,278 & 313,297 & 10,241 & 9,883 & 10,913 \\
\hline 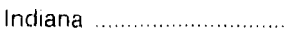 & 93,025 & 98,906 & 101.670 & 3,113 & 3,740 & ค 3.977 \\
\hline 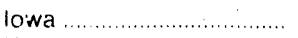 & 51,155 & 54,076 & 54,251 & 1.543 & 1,648 & 2,432 \\
\hline 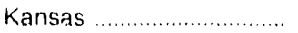 & 53.471 & $54,2,85$ & 56,239 & 1,684 & 1,825 & 2,479 \\
\hline 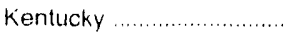 & 35,713 & 41,354 & 43,726 & 1,287 & 1,375 & A 1,598 \\
\hline 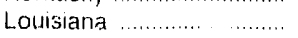 & 45,079 & 39,278 & 44,260 & 2,287 & 2,350 & P 2,624 \\
\hline Maıne .................................. & 414 & 441 & 398 & 19 & 20 & 27 \\
\hline 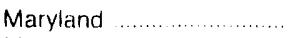 & 49,439 & 52,619 & 53,550 & 2,019 & 2,240 & 2,696 \\
\hline Massachusetts ................... & 78,463 & 80.202 & 78,903 & 2,639 & 3,166 & R 4.899 \\
\hline 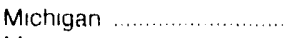 & 219,148 & 240,001 & 245,246 & 8,299 & $7,82.3$ & 10,750 \\
\hline 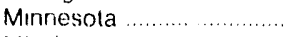 & 67.775 & 77.608 & 77,037 & 2,284 & 2,355 & A 3,309 \\
\hline Mississıppı ...................... & 25,616 & 18,201 & 19,768 & 1.115 & 1,173 & 1,299 \\
\hline 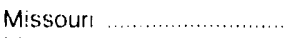 & 33,104 & 89,957 & 92,002 & 2.711 & 2.944 & A 3.781 \\
\hline 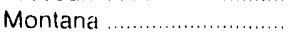 & 10.711 & 12,297 & 11,217 & 395 & ค 392 & ค 706 \\
\hline 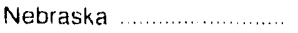 & 28.098 & 30,536 & 31,422 & 947 & 1,056 & R 1.294 \\
\hline 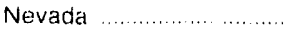 & 11,079 & 11.748 & 11,132 & 520 & 562 & 706 \\
\hline New Hampshıre .............. & 4.171 & 4.474 & 4,233 & 128 & 145 & 247 \\
\hline New Jersey .................... & 116.405 & 132,264 & 129,164 & 4,582 & 5,092 & 5,799 \\
\hline New Mexico ....................... & 22.128 & 19,208 & 20,652 & 771 & 812 & 1,116 \\
\hline 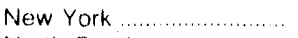 & 241,749 & 253,272 & 250.504 & 8.758 & 10,055 & A 13,590 \\
\hline North Carolina ................. & 25,090 & 26,884 & 27,061 & 733 & 765 & 953 \\
\hline North Dakota .................. & 6,077 & 6.960 & 6,456 & 167 & 191 & 314 \\
\hline 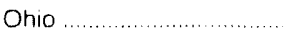 & 199,909 & 221,317 & 233,748 & 7.023 & 7,744 & 9,442 \\
\hline 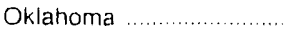 & 49,342 & 51,499 & 53.755 & 1.616 & 1,697 & 2,234 \\
\hline 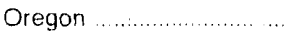 & 15,468 & 16.399 & 15,231 & 561 & 706 & 1,118 \\
\hline 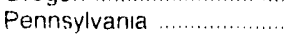 & 170,846 & 183,412 & 188,338 & 5,759 & 6,037 & ค 8,484 \\
\hline 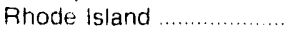 & 12,684 & 12,862 & 12,633 & 481 & 482 & ค 762 \\
\hline South Carolına .................. & 13,376 & 14,068 & 14,852 & 347 & 367 & 447 \\
\hline South Dakota ................ & 6,567 & 7,670 & 7.671 & 215 & 231 & 326 \\
\hline 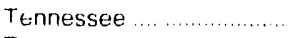 & 32,313 & 34,272 & 34.629 & 957 & 988 & 1,258 \\
\hline 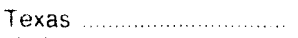 & 185,366 & 151,758 & 151,634 & 10,261 & 10,398 & A 11,617 \\
\hline Utan & 28,446 & 32,564 & 30.222 & 1,236 & 1,314 & ค 1,880 \\
\hline 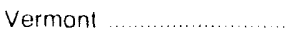 & 1.370 & 1,468 & 1,305 & 40 & 43 & 72 \\
\hline Virgınıa & 35,775 & 41.235 & 40.704 & 1,425 & 1,450 & 1.712 \\
\hline Washıngton & 25,059 & 27.192 & 24,301 & 794 & 1,048 & 1,770 \\
\hline West Virginia .................... & 23,696 & 24,946 & 26,295 & 695 & 723 & ค 1,054 \\
\hline 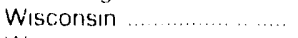 & 74,826 & 86,540 & 83,197 & 2,556 & 2,380 & 3,300 \\
\hline 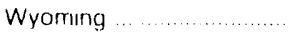 & 8,171 & 8,527 & 8.421 & 288 & 335 & 579 \\
\hline$\ldots \ldots \ldots$ & $3,034,237$ & $3.213,825$ & 3.250 .929 & 124,099 & म 129,326 & A 162,298 \\
\hline
\end{tabular}

See frotnote at and of tatsle. 
Table 22. Natural Gas Deliveries to Residential Consumers by State (Continued) (Million Cubic Feet)

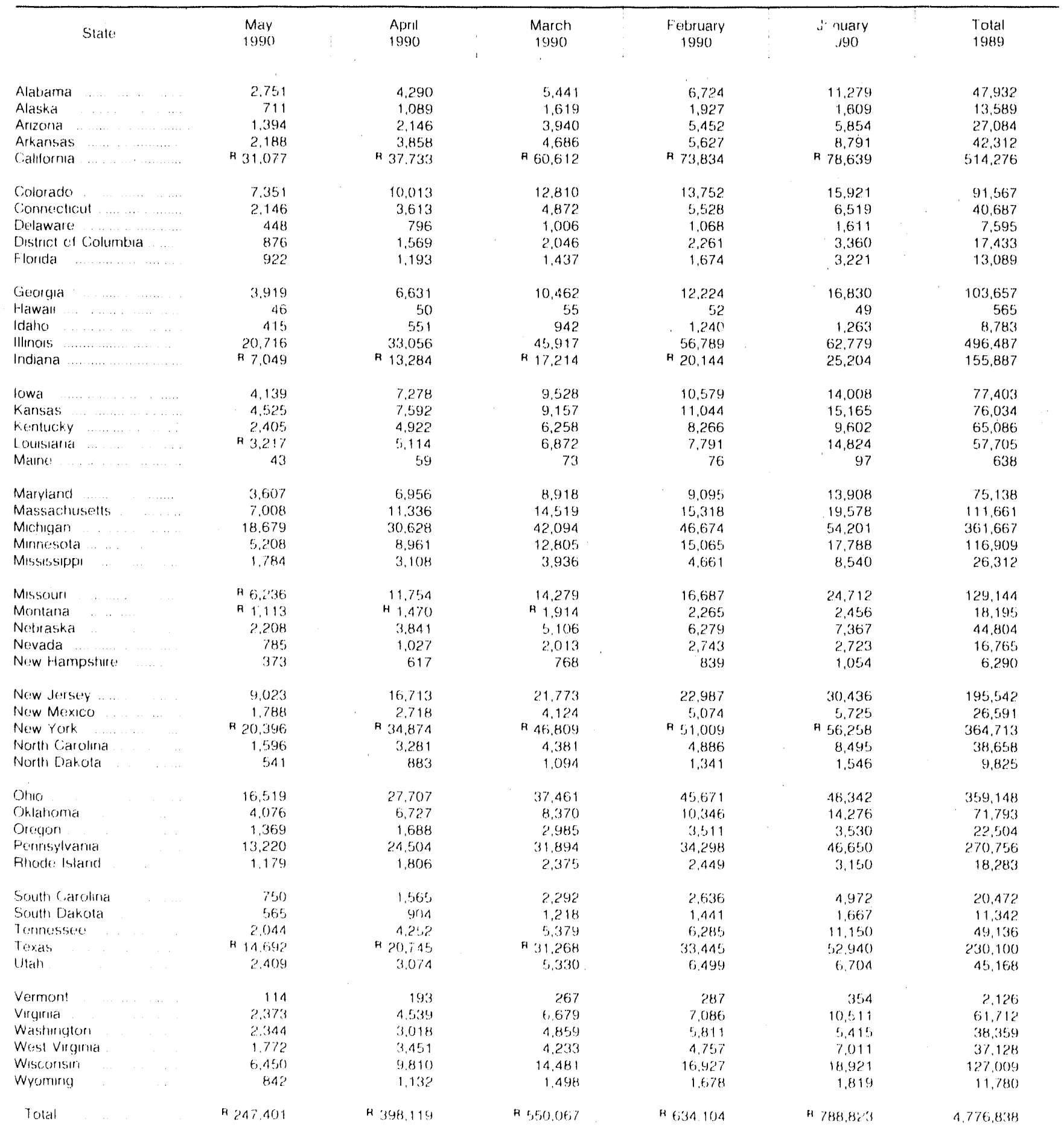

Son footrote at sud of tatbe: 
Table 22. Natural Gas Deliveries to Residential Consumers by State (Continued) (Million Cubic Feet)

\begin{tabular}{|c|c|c|c|c|c|c|c|}
\hline State & $\begin{array}{c}\text { December } \\
1989\end{array}$ & $\begin{array}{c}\text { November } \\
1989\end{array}$ & & $\begin{array}{c}\text { Cotober } \\
1989\end{array}$ & $\begin{array}{c}\text { September } \\
1989\end{array}$ & $\begin{array}{c}\text { August } \\
1989\end{array}$ & $\begin{array}{l}\text { July } \\
1989\end{array}$ \\
\hline Alabama & 7,212 & 3,632 & & 1.854 & 1,399 & 1,377 & 1.451 \\
\hline Alaska & 1,656 & 1.474 & & 895 & 536 & 370 & 432 \\
\hline 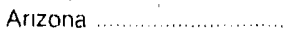 & 3.945 & 1.768 & & 959 & 833 & 777 & 880 \\
\hline 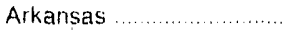 & 6,356 & 2,987 & & 1,562 & 1,239 & 1,137 & 1,237 \\
\hline 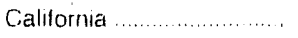 & 63.104 & 40,070 & & 28,083 & 24,184 & 22,977 & 22,730 \\
\hline Colorado ........................... & 11.066 & 6,975 & & 3,750 & 2,601 & 2,279 & 2.778 \\
\hline Connecticut . ...................... & 6,991 & 3,221 & & 1,986 & 1,138 & 996 & 1,114 \\
\hline 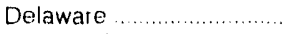 & 1,085 & 485 & & 275 & 207 & 189 & 192 \\
\hline District of Columbia ......... & 2,637 & 1.099 & & 655 & 494 & 483 & 507 \\
\hline 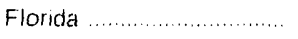 & 1.825 & 974 & & 637 & 641 & 617 & 674 \\
\hline 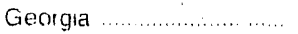 & 22.132 & 11,108 & & 5,189 & 3,465 & 2,791 & 2,987 \\
\hline 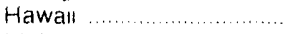 & 46 & 46 & & 43 & 45 & 42 & 46 \\
\hline 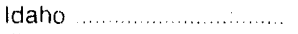 & 1,241 & 768 & & 329 & $22 i$ & 146 & 160 \\
\hline 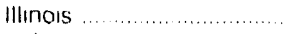 & 93,702 & 51,130 & & 25,845 & 13,532 & 9,889 & 10,700 \\
\hline Indiana & 29.647 & 15,182 & & 8,195 & 3,956 & 2,868 & 2,893 \\
\hline lowa $\ldots \ldots \ldots \ldots \ldots \ldots \ldots \ldots \ldots \ldots \ldots$ & 12,401 & 5,912 & & 3,225 & 1,790 & 1,527 & 1,647 \\
\hline 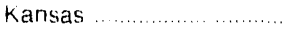 & 11.694 & 5,398 & & 2,901 & 1,955 & 1.687 & 1,845 \\
\hline 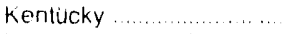 & 13,034 & 6.141 & & 3,109 & 1,446 & 1.337 & 1,332 \\
\hline 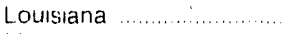 & 9,714 & 4,166 & & 2,513 & 2,035 & 2,026 & 2,189 \\
\hline Maine ............................... & 95 & 46 & & 35 & 21 & 19 & 12 \\
\hline 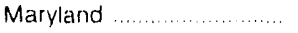 & 12,076 & 5,131 & & 3,134 & 2,177 & 2,070 & 2,241 \\
\hline Massachusetts & 16.224 & 7,495 & & 4,839 & 2,901 & 2.683 & 3,006 \\
\hline Michıgan ...................... & 60,266 & 31,679 & & 19.994 & 9.726 & 7,957 & 8,052 \\
\hline Minnesota ........................ & 20,120 & 11,067 & & 5,430 & 2,682 & 2,269 & 2,388 \\
\hline Mississippi ..................... & 4,241 & 1,933 & & 1,043 & 894 & 829 & 864 \\
\hline Missouri ….................. & 21,588 & 9,441 & & 5,047 & 3,111 & 2,732 & 3,008 \\
\hline 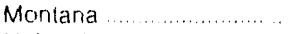 & 2,518 & 1,728 & & 1,084 & 568 & 435 & 426 \\
\hline Nebraska & 7.297 & 3.788 & & 1.972 & 1,212 & 1,007 & 1,078 \\
\hline 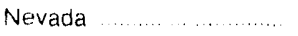 & 2,359 & 1,400 & & 703 & 555 & 478 & 531 \\
\hline New Hampshire ............... & 926 & 448 & & 274 & 169 & 127 & 155 \\
\hline New Jersey $\ldots . . . . . . . . . . . . .$. & 33,347 & 15.715 & & 8,933 & 5.283 & 4.778 & 5,231 \\
\hline New Mexico ............... & 3.746 & 1,975 & & 899 & 765 & 734 & 829 \\
\hline 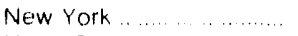 & 57,291 & 28,576 & & 16,060 & 9,514 & 9,189 & 10,098 \\
\hline North Carolina $\ldots . . . . . . . . . . . .$. & 6,866 & 2,823 & & 1,338 & 745 & 695 & 744 \\
\hline North Dakota ............... & 1.411 & 816 & & 398 & 240 & 166 & 213 \\
\hline Ohio $\ldots \ldots \ldots \ldots$ & 69,403 & 37,970 & & 20,647 & 9,812 & 7,262 & 7,332 \\
\hline 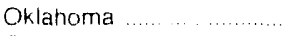 & 11,039 & 4,798 & & 2,571 & 1,886 & 1,654 & 1,858 \\
\hline 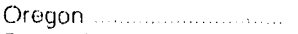 & 2.886 & 1,835 & & 804 & 581 & 549 & 696 \\
\hline Pennsylvana $\ldots . . . . . . . . . . .$. & 44,962 & 22,443 & & 13,222 & 6.717 & 6,014 & 6,401 \\
\hline 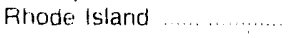 & 2.744 & 1,325 & & 855 & 497 & 464 & 500 \\
\hline South Carolına ............... & 3.911 & 1.526 & & 620 & 346 & 327 & 395 \\
\hline South Dakota $\ldots . . . . . . . . .$. & 1,764 & 1.076 & & 535 & 2.98 & 221 & 245 \\
\hline Tennessee $\ldots \ldots \ldots \ldots \ldots$ & 8,590 & 3,551 & & 1.681 & 1,043 & 960 & 1,040 \\
\hline 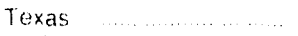 & 43.770 & 16,927 & & 9,777 & 7.869 & 7,211 & 7,726 \\
\hline Utah $\ldots \ldots \ldots \ldots \ldots \ldots \ldots$ & 5,923 & 3.640 & & 1.718 & 1.322 & 1.235 & 1,374 \\
\hline Vermont & 353 & 165 & & 97 & 43 & 37 & 40 \\
\hline Virguna $\ldots . . .$. & 11,473 & 4.799 & & 2,831 & 1,374 & 1,340 & 1,479 \\
\hline Washmgton & 5,091 & 3,484 & & 1,622 & 969 & 801 & 1,002 \\
\hline West Virginia ............. & 6.382 & 3.240 & & 1,738 & 824 & 695 & 741 \\
\hline Wisconsin & 20,661 & 10,581 & & 6,052 & 3,173 & 2.220 & 2,736 \\
\hline Wyoming & 1.412 & 973 & & 518 & 350 & 259 & 344 \\
\hline Total & 790.223 & 404,930 & & 228,478 & 139,383 & $120,93 ?$ & 128,582 \\
\hline
\end{tabular}

See footnote al end of table. 
Table 22. Natural Gas Deliveries to Residential Consumers by State (Continued) (Million Cubic Feet)

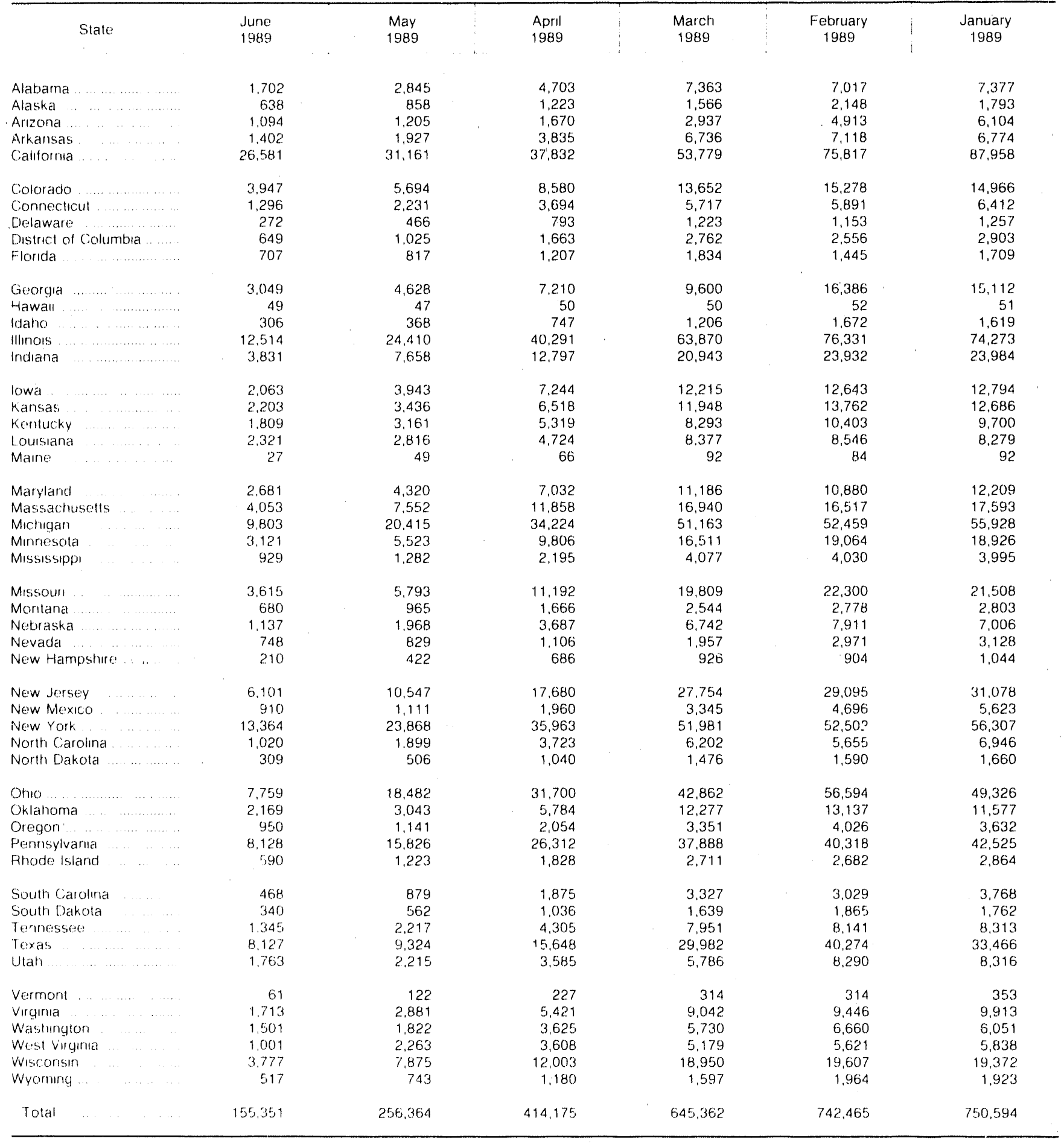

R Revised Data

Notes and Sources see the last page of this section 
Table 23. Natural Gas Deliveries to Commercial Consumers by State (Million Cubic Feet)

\begin{tabular}{|c|c|c|c|c|c|c|}
\hline State & $\begin{array}{l}\text { YTD } \\
1990\end{array}$ & $\begin{array}{l}\text { YTD } \\
1989\end{array}$ & $\begin{array}{l}\text { YTD } \\
1988\end{array}$ & $\begin{array}{c}\text { August } \\
1990\end{array}$ & $\begin{array}{l}\text { July } \\
1990\end{array}$ & $\begin{array}{l}\text { June } \\
1990\end{array}$ \\
\hline 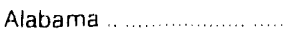 & 17.293 & 18.414 & 18,450 & 1,214 & 1.196 & 993 \\
\hline 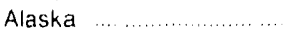 & 13,507 & 14,387 & 13,596 & 1.077 & 1,061 & 1,087 \\
\hline Arizonla .............................. & 20,311 & 19,953 & 20,052 & $1,5,81$ & 1,686 & 1,835 \\
\hline 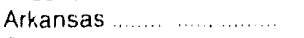 & 17,997 & 19,049 & 20,208 & 1,044 & 1.031 & 1,073 \\
\hline 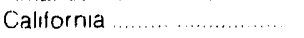 & 191.175 & 171,953 & 170.794 & 17,659 & ค 21,061 & A 17,410 \\
\hline 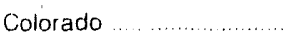 & 48,882 & 48,399 & 49,805 & 2,123 & 2.162 & 3,162 \\
\hline 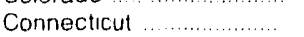 & 18,609 & 21,330 & 18,717 & 1,309 & 1,352 & 1,314 \\
\hline 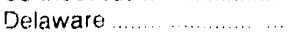 & 2,888 & 2,935 & 2,905 & 140 & 140 & 170 \\
\hline District of Columbia & 9,619 & 11,112 & 10,691 & 589 & 528 & 686 \\
\hline 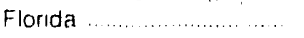 & 25,749 & 23,690 & 26,310 & 2.481 & 2,564 & 2,857 \\
\hline$\ldots \ldots \ldots \ldots \ldots \ldots \ldots$ & 34,839 & 33,817 & 37.774 & 2.539 & 2,365 & 2,638 \\
\hline Hawall & 1,448 & 1,425 & 1,392 & 180 & 195 & 181 \\
\hline Idaho ... & 5,225 & 6.287 & 5.669 & 279 & 293 & 357 \\
\hline Illinols & 127,747 & 124,857 & 143.871 & 5,863 & 5,765 & 5,741 \\
\hline 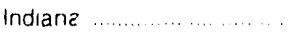 & 43,819 & 46,360 & 47.378 & 1,861 & 1,659 & A 1,965 \\
\hline 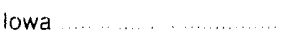 & 29,933 & 31.919 & 31,738 & 1,196 & 1,217 & 1,549 \\
\hline 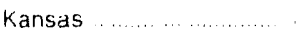 & 42,607 & 41.540 & 44.405 & 4,752 & 5,111 & 3,185 \\
\hline 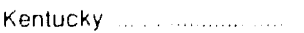 & 20,386 & 23,052 & 24,298 & 1.073 & 1,033 & R 1,146 \\
\hline Loussiana & 20,532 & 18.228 & 19,895 & 1.411 & 1.551 & A 1,708 \\
\hline Maine . . . . . . . . & 1.032 & 1.106 & 995 & 47 & 52 & 62 \\
\hline 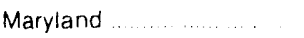 & 17,333 & 19.273 & 18,358 & 986 & 1,075 & 1,116 \\
\hline Massachusetts $\ldots . . . . . . . . . .$. & 34,998 & 36.477 & 34.652 & 2,051 & 2,020 & ค 2,504 \\
\hline 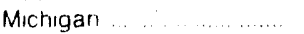 & 107,076 & 118,233 & 117.742 & 4.999 & 4,743 & 5,495 \\
\hline Minnesola ............... & 48,524 & 55,565 & 55,181 & 2,071 & 1,997 & ค 2.391 \\
\hline Mississippi $\ldots \ldots \ldots$ & 17.944 & 11,797 & 12.879 & 1.202 & 1,262 & 1,404 \\
\hline$\ldots \ldots \ldots$ & 41,532 & 43.603 & 45,281 & 1,918 & 1,980 & A 2,260 \\
\hline 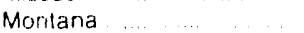 & 7.688 & 8.877 & 7,948 & 350 & R 325 & R 490 \\
\hline Nebraska & 26,058 & 26,810 & 29,528 & 2,595 & 4,829 & ค 1,326 \\
\hline Nevada $\ldots$ & 10.793 & 10.403 & 10,401 & 1,193 & 928 & 953 \\
\hline New Hampshire $\ldots$ & 3,461 & 3.738 & 3,513 & 153 & 153 & 207 \\
\hline New Jersey & 71.060 & 80.959 & 71.915 & 4,901 & 4,912 & 4,685 \\
\hline New Mexico & 17.278 & 19.964 & 22,368 & 863 & 825 & 1,082 \\
\hline New york. & 132,023 & 131.546 & 129,183 & 9,564 & 9,634 & R 10,377 \\
\hline North Carolina & 19.854 & 22.732 & 22,074 & 1,385 & 1,302 & 1,342 \\
\hline Noriti Dakota _........... & 6.519 & 7,349 & 6,848 & 297 & 314 & 353 \\
\hline 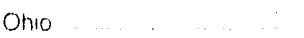 & 95,630 & 102.082 & 106,701 & 4,138 & 3,948 & 4,721 \\
\hline Oklahoma $\ldots$ & 33,124 & 27,315 & 34,453 & 1,354 & 1,312 & 1,886 \\
\hline Oregon & 12.932 & 14.408 & 13,106 & 765 & 817 & 1,046 \\
\hline Penrisylvania & 83,668 & 88.340 & 88,251 & 4,524 & 4,441 & P 5,195 \\
\hline Fhode Island & 5.236 & 5,880 & 5,850 & 355 & 413 & ค 244 \\
\hline Soutn Carolina & 11.096 & 11.400 & 11,931 & 838 & 843 & 903 \\
\hline South Dakota & 5.357 & 5.911 & 5,887 & 217 & 221 & 281 \\
\hline Tennessee & 29.470 & 32,182 & 32,298 & 1.779 & 1,644 & 1,906 \\
\hline Texas & 158.843 & 127,234 & 124.620 & 15,610 & ค 18,936 & A 19,083 \\
\hline Utah & 12134 & 11.994 & 13,329 & 364 & 373 & ค 581 \\
\hline Vermorit & 1.297 & 1,382 & 1.302 & 62 & 46 & 63 \\
\hline Virquna & 28.044 & 29.918 & 29,014 & 1,805 & 1,832 & 1.771 \\
\hline Wasturigtor & 25.235 & 27.264 & 25,586 & 1,474 & 1,637 & 1.857 \\
\hline West virgirila & 13.828 & 15.285 & 15.244 & 782 & 752 & R 937 \\
\hline Wiscorisini & 43.588 & 47.367 & 46,603 & 1.707 & 1,757 & 1.918 \\
\hline Wyoming & 6.083 & 6.141 & 6,360 & 237 & 265 & 420 \\
\hline Tolal & $1.8 ? 1.298$ & 1.831 .236 & 1.857 .338 & 118.965 & A 127,557 & A 127,913 \\
\hline
\end{tabular}

See toolnote al arid of lable 
Table 23. Natural Gas Deliveries to Commercial Consumers by State (Continued) (Million Cubic Feet)

\begin{tabular}{|c|c|c|c|c|c|c|}
\hline Slate & $\begin{array}{l}\text { May } \\
1990\end{array}$ & $\begin{array}{l}\text { April } \\
1990\end{array}$ & $\begin{array}{c}\text { March } \\
1990\end{array}$ & $\begin{array}{c}\text { February } \\
1990\end{array}$ & $\begin{array}{c}\text { January } \\
1990\end{array}$ & $\begin{array}{l}\text { Total } \\
1989\end{array}$ \\
\hline 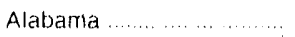 & 1.628 & 2,162 & 2,624 & 3,006 & 4,470 & 26,438 \\
\hline Alaska ...................... & 1.396 & 1.734 & 2.329 & 2,477 & 2,346 & 21,738 \\
\hline Arizona & 2,424 & 2,347 & 3,169 & 3,564 & 3,705 & 28,580 \\
\hline 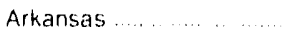 & 1.407 & 2,238 & 2.825 & 3,390 & 4,989 & 27,284 \\
\hline Calıforna $\ldots \ldots \ldots \ldots \ldots$ & R 18.552 & म 23,439 & A 27,435 & A 34,812 & A 30,807 & 259,118 \\
\hline 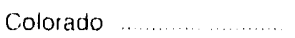 & 5,181 & 6.989 & 8,743 & 9.668 & 10,854 & 67,477 \\
\hline 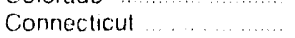 & 1,564 & 2,673 & 3,146 & 3,426 & 3,825 & 30,781 \\
\hline 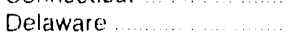 & 233 & 408 & 517 & 516 & 764 & 4,184 \\
\hline District of Columbia .... & 933 & 1,358 & 1.755 & 1,822 & 1,948 & 15,741 \\
\hline Florida $\ldots \ldots \ldots \ldots$ & 3,058 & 3,272 & 3.509 & 3,573 & 4,435 & 35,105 \\
\hline 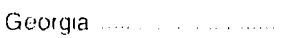 & 3,001 & 4.166 & 5,368 & 6,229 & 8,533 & 53,114 \\
\hline 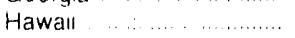 & 174 & 173 & 181 & 181 & 183 & 2,129 \\
\hline 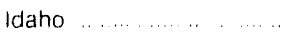 & 439 & 559 & 917 & 1,177 & 1,204 & 8,994 \\
\hline llinois & 10,096 & 17.945 & 22,690 & 27,016 & 32,631 & 196,133 \\
\hline 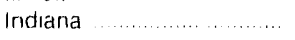 & ค 3.193 & ค 6,091 & ค 7.966 & R 9,387 & 11,697 & 73,613 \\
\hline lowa $\ldots$ & 2,330 & 4.122 & 5,532 & 6,165 & 7,822 & 46,142 \\
\hline 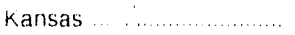 & 3,456 & 5,228 & 5,548 & 6,715 & 8,612 & 58,554 \\
\hline Kentucky .................. & 1,575 & 2,690 & 3,464 & 4,229 & 5,176 & 36,145 \\
\hline 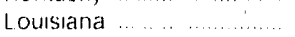 & A 1,855 & 2.511 & 2,928 & 3,243 & 5,325 & 26,617 \\
\hline Maine $\ldots \ldots \ldots \ldots$ & 95 & 154 & 186 & 200 & 236 & 1,650 \\
\hline 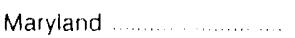 & 1.535 & 2,444 & 3.016 & 3,074 & 4,087 & 26,920 \\
\hline Massachusetts ............. & 3.227 & 4.997 & 6.077 & 6,250 & 7.872 & 51,508 \\
\hline Michigan & 8.936 & 14,889 & 20.094 & 21,945 & 25,975 & 176,181 \\
\hline 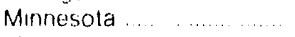 & 3.801 & 6,363 & 9,273 & 10.712 & 11.916 & 85,183 \\
\hline 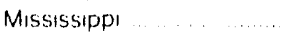 & 1.695 & 2,246 & 2.442 & 2,936 & 4,757 & 17,568 \\
\hline Missouri & R 3,250 & 5,617 & 6.848 & 7,987 & 11,672 & 63,039 \\
\hline 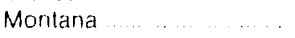 & B 771 & R 1,005 & A 1,411 & 1,573 & 1,763 & 13.141 \\
\hline Netraska ......... & 1,561 & 2,959 & 3,535 & 4,234 & 5,019 & 37,351 \\
\hline Nevada & 989 & 1.165 & 1,710 & 1,929 & 1.926 & 15,116 \\
\hline New Hampshure ............ & 288 & 485 & 579 & 753 & 843 & 5,371 \\
\hline New Jersey .... & 6.298 & 10,120 & 12,388 & 12.426 & 15,330 & 117.385 \\
\hline New Mexico & 1.508 & 2,173 & 3,068 & 3.694 & 4.065 & 28,459 \\
\hline New York & P 11,466 & ค 18,013 & ค 23,236 & ค 23.791 & R 25,942 & 196,380 \\
\hline North Caroltna $\ldots . . . .$. & 1,649 & 2.520 & 3.165 & 3,444 & 5,047 & 33.145 \\
\hline North Dakota & 567 & 966 & 1,145 & 1,342 & 1,535 & 10.609 \\
\hline Onio $\ldots \ldots \ldots$ & 7,256 & 13.011 & 19,785 & 19,091 & 23,680 & 161,516 \\
\hline Oklahorna $\ldots \ldots \ldots$ & 2,826 & 4,238 & 5,176 & 6.955 & 9,377 & 38,493 \\
\hline 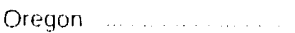 & 1,147 & 1,402 & 2,317 & 2,740 & 2,698 & 20,249 \\
\hline Pennsylvaria & 6.6 .37 & 12,117 & 14.718 & 15,928 & 20,108 & 132,500 \\
\hline Rhode Istand. & 549 & 816 & 851 & 860 & 1,138 & 8,767 \\
\hline South Garolina ............ & 1,123 & 1,344 & 1.678 & 1.755 & 2.612 & 16,525 \\
\hline South Dakola ............... & 461 & 722 & 998 & 1.151 & 1,306 & 8,826 \\
\hline Tennessee ....... . .......... & 2.371 & 3.634 & 4,359 & 5,524 & 8,253 & 47,573 \\
\hline Texas & ค 16,353 & R 18,719 & ค 22,259 & 20,678 & 27,205 & 184,179 \\
\hline$\ldots \ldots \ldots \ldots$ & 932 & 1.304 & 2.462 & 3.013 & 3,105 & 16,522 \\
\hline Vermont & 108 & 183 & 250 & 269 & 316 & 2,081 \\
\hline Virgınia $\ldots \ldots \ldots \ldots$ & 2,332 & 4,413 & 4,943 & 5,003 & 5.945 & 44,181 \\
\hline Wastungton .... & 2,534 & 3.128 & 4.275 & 5,387 & 4.883 & 38.502 \\
\hline West virginis & 1.169 & 1.947 & 2.240 & 2,520 & 3,481 & 23,257 \\
\hline Wiscorisin & 3,620 & 5,305 & 8.524 & 9.180 & 11,577 & 70,090 \\
\hline Wyoming & 643 & 843 & 1,110 & 1,228 & 1.337 & 8.551 \\
\hline$\ldots \ldots \ldots$ & B 160.253 & A 239,348 & ค 304,765 & R 338.168 & B 404,329 & $2,718,716$ \\
\hline
\end{tabular}

See footnote al end of table 
Table 23. Natural Gas Deliveries to Commercial Consumers by State (Continued) (Million Cubic Feet)

\begin{tabular}{|c|c|c|c|c|c|c|}
\hline State & $\begin{array}{c}\text { December } \\
1989\end{array}$ & $\begin{array}{c}\text { November } \\
1989\end{array}$ & $\begin{array}{c}\text { Oclober } \\
1989\end{array}$ & $\begin{array}{c}\text { September } \\
1989\end{array}$ & $\begin{array}{l}\text { August } \\
1989\end{array}$ & $\begin{array}{l}\text { July } \\
1989\end{array}$ \\
\hline 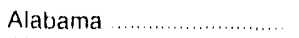 & 3,503 & 1,953 & 1,381 & 1,187 & 1,115 & 1,672 \\
\hline 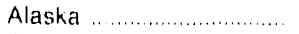 & 2.429 & 2.144 & 1,577 & 1,202 & 1.091 & 1,042 \\
\hline 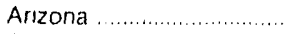 & 3,106 & 2,294 & 1,633 & 1,596 & 1,578 & 1.804 \\
\hline 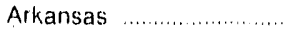 & 3,943 & 1,949 & 1.246 & 1,097 & 1,008 & 1.020 \\
\hline California ............................ & 26,192 & 21,665 & 19,856 & 19.452 & $17,35,1$ & 15,319 \\
\hline 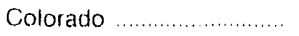 & 8,102 & 5.441 & 3,232 . & 2,303 & 2,110 & 2,328 \\
\hline Connecticut ........................ & 3,891 & 2,324 & 1.685 & 1,551 & 1.579 & 1,528 \\
\hline 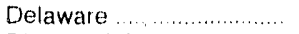 & 630 & 294 & 182 & 144 & 133 & 128 \\
\hline Districl of Columbia ......... & 2,052 & 1,090 & 775 & 712 & 763 & 692 \\
\hline Florida $\ldots . . . \ldots \ldots \ldots \ldots$ & 3,620 & 2,868 & 2,444 & 2,484 & 2,369 & 2,525 \\
\hline 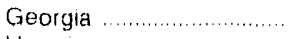 & 8.393 & 4,836 & 3,477 & 2.590 & 2,361 & 2,412 \\
\hline 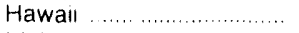 & 177 & 172 & 179 & 175 & 173 & 182 \\
\hline 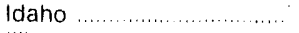 & 1.172 & 783 & 433 & 320 & 249 & 300 \\
\hline 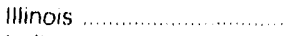 & 33,840 & 20,169 & 11,091 & 6,176 & 4,862 & 5,444 \\
\hline 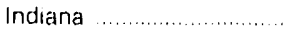 & 13,611 & 7,576 & 3,850 & 2.217 & 1,659 & 1.579 \\
\hline lowa ....... & 7,110 & 3,738 & 2.129 & 1,247 & 1,234 & 1.190 \\
\hline 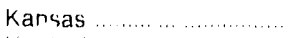 & 6.763 & 4,386 & 2,785 & 3,081 & 4,812 & 3,566 \\
\hline 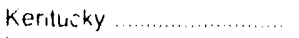 & 6.648 & 3.327 & 1.971 & 1.147 & 991 & 1,099 \\
\hline 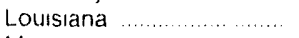 & 3.454 & 1,995 & 1,537 & 1,405 & 1,314 & 1,372 \\
\hline Marne .............................. & 269 & 128 & 96 & 60 & 50 & 51 \\
\hline Maryland …....................... & 3,647 & 1,801 & 1.220 & 978 & 941 & 968 \\
\hline Massachuselts ................ & 6.864 & 3.647 & 2,720 & 1,799 & 1,894 & 1,726 \\
\hline Michıgan ........................ & 28,269 & 15.776 & 8,855 & 5,047 & 4,467 & 4,570 \\
\hline 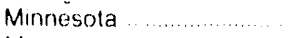 & 13,661 & 8,764 & 4,718 & 2,475 & 2,011 & 2,112 \\
\hline Mississippt ..................... & 2.727 & 1,350 & 939 & 754 & 701 & 718 \\
\hline 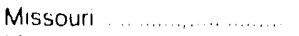 & 9,903 & 4,716 & 2,798 & 2,021 & 1,7830 & 1,829 \\
\hline 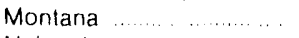 & 1,781 & 1,235 & 806 & 442 & 381 & 330 \\
\hline Nebraska .......................... & 4,196 & 2,602 & 2,109 & 1,631 & 4.240 & 3,381 \\
\hline Nevada ........... & 1,690 & 1,257 & 954 & 811 & 805 & 790 \\
\hline New Hampshire & 817 & 393 & 248 & 176 & 140 & 154 \\
\hline New Jersey................. & 16,342 & 9.150 & 6.297 & 4,638 & 4,520 & 4,881 \\
\hline 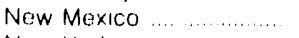 & 3.830 & 2,441 & 1.191 & 1,034 & 1.146 & 913 \\
\hline 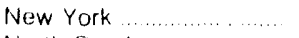 & 27.650 & 16,631 & 11,741 & 8.812 & 8,305 & $8,382$. \\
\hline North Carolına ............... & 4.396 & 2,639 & 1.843 & 1,534 & 1.454 & 1,475 \\
\hline North Dakota .................. & 1.506 & 951 & 464 & 340 & 238 & 269 \\
\hline Ohio $\ldots \ldots \ldots \ldots \ldots \ldots$ & 30.143 & 15,949 & 8,946 & 4.397 & 3,735 & 3,565 \\
\hline Oklahoma $\ldots . . . . . . . . . .$. & 5,871 & $2,5 \cdot 41$ & 1,527 & 1,241 & 1,097 & 1,180 \\
\hline Oregon $\ldots \ldots \ldots \ldots$ & 2.363 & 1,624 & 972 & 882 & 804 & 823 \\
\hline Pennsylvanıa ............... & 21,010 & 11.588 & 7.185 & 4.378 & 4,161 & 4.272 \\
\hline Rhode Island ................. & 1.014 & 654 & 657 & 560 & 518 & 471 \\
\hline South Carolina & 1,964 & 1,324 & 970 & 866 & 808 & 908 \\
\hline South Dakota .............. & 1.374 & 851 & 434 & 256 & 197 & 203 \\
\hline Tennessee $\ldots \ldots \ldots$ & 7.414 & $, 3,774$ & 2,358 & 1.845 & 1.661 & 1.804 \\
\hline 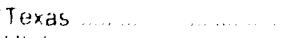 & 24.013 & 12,961 & 10,462 & 9,509 & 11.439 & 12,862 \\
\hline Utah $\quad \ldots \ldots$ & 2.304 & 1,313 & $52 ?$ & 390 & 346 & 357 \\
\hline Vermont $\ldots \ldots . . . .$. & 307 & 189 & 130 & 73 & 57 & 57 \\
\hline Vugını & 6.474 & 3.612 & 2.192 & 1.985 & 1.485 & 1,866 \\
\hline Wastungton & 4.433 & 3,366 & 2.083 & 1.357 & 1,548 & 1,671 \\
\hline West Virgina & 3,704 & 2.036 & 1.373 & 859 & 853 & $78 t$ \\
\hline Wisconsin & 11,182 & 6,386 & 3,437 & 1.718 & 1,499 & 1.562 \\
\hline Wyoming & 1.022 & 124 & 396 & $26 j 8$ & 2333 & 285 \\
\hline Total & 390.776 & 231.374 & 152,108 & 113222 & 110.276 & 110.221 \\
\hline
\end{tabular}

See tootriole at end of lable 
Table 23. Natural Gas Deliveries to Commercial Consumers by State (Continued) (Million Cubic Feet)

\begin{tabular}{|c|c|c|c|c|c|c|}
\hline Slate & $\begin{array}{l}\text { Juno } \\
1989\end{array}$ & $\begin{array}{l}\text { May } \\
1989\end{array}$ & $\begin{array}{l}\text { April } \\
1989\end{array}$ & $\begin{array}{c}\text { March } \\
1989\end{array}$ & $\begin{array}{c}\text { Fobruaty } \\
1969\end{array}$ & $\begin{array}{c}\text { January } \\
1989\end{array}$ \\
\hline Alabama ................. & 1.213 & 1.717 & 2.366 & 3,391 & 3,510 & 3,430 \\
\hline Alaska $\ldots \ldots \ldots$ & 1.287 & 1,569 & 1.949 & 2,258 & 2,601 & 2500 \\
\hline Arizona $\ldots . . . . . .$. & 1,050 & 1.992 & 2.274 & 2.843 & 3,570 & 3,942 \\
\hline Aikansas $\ldots$ & 1,357 & 1.261 & 2,181 & 3,963 & 4,338 & 3,921 \\
\hline Callifornia & 21,469 & 19,249 & 18,656 & 21,880 & 29,564 & 28,465 \\
\hline 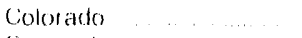 & $\{1,08\}^{\prime}$ & 4.160 & $6,1,15$ & 9,208 & 10,845 & $10,5 ? 2$ \\
\hline Conneclicut $\quad \ldots \quad \ldots$. & 1.625 & 2,106 & 2,8497 & $3,93 \%$ & 3,749 & 3,009 \\
\hline Dolaware & 166 & 249 & 394 & 624 & 605 & 632 \\
\hline Disirici of Columba & 803 & 499 & 1.635 & 2,066 & 2,021 & 2.133 \\
\hline Fornda $\quad \ldots \ldots \ldots$ & 2.570 & 2.670 & 3083 & 3,545 & 3,435 & $3,49,3$ \\
\hline$\ldots \ldots \ldots$ & 2.341 & 2.983 & 3,922 & 5,166 & 7.502 & 7.130 \\
\hline Hawäl $\ldots$ & 175 & 181 & 175 & 171 & 178 & 187 \\
\hline laho $\ldots \ldots$ & 353 & 408 & 690 & 1.156 & 1,569 & 1,562 \\
\hline Illmous $\ldots \ldots \ldots \ldots \ldots \ldots$ & 5,974 & 9.306 & 15,896 & 25,958 & 29,585 & 27,832 \\
\hline Indiana ...................... & 1,904 & 3,296 & 5,767 & 9,612 & 11,371 & 11,169 \\
\hline lowa $\ldots \ldots \ldots \ldots \ldots$ & 1,380 & 2.216 & 4,133 & 6,928 & 7.166 & $7,37 ?$ \\
\hline Kansas .............. & 2.547 & 3,660 & 5,433 & 6.870 & 7,697 & 7,155 \\
\hline kentucky ... ................ & 1.167 & 1,766 & 2,839 & 4,546 & 6,506 & 5,139 \\
\hline Loustaná $\ldots \ldots \ldots$ & 1,449 & 1,754 & 2,315 & 3,394 & 3,298 & 3,332 \\
\hline Maine ..... . & 6.3 & 106 & 160 & 221 & 226 & 229 \\
\hline 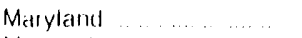 & 1,154 & 1.701 & 2,586 & 4,217 & 3,700 & 3.976 \\
\hline Matssachusetts $\quad . . . . . . . . .$. & 2,150 & 3,703 & $5,39 ?$ & 7,234 & 6,0834 & 7,394 \\
\hline Michnyan $\quad \ldots \ldots \ldots$ & 5.954 & 10.150 & 16,375 & 24.717 & 25,448 & 26,552 \\
\hline Mirinesola & 2.416 & 3.980 & 6,916 & 11,411 & 13,607 & 13,112 \\
\hline Missisisippl & 739 & 947 & 1.407 & 2.411 & 2,502 & 2,372 \\
\hline Missouri & $2,35 ! 9$ & 2,799 & 5.246 & 9,192 & 10.280 & 10,118 \\
\hline.$\quad$. & 178 & 687 & 1.208 & 1.841 & 1.923 & 2.029 \\
\hline Nobraskal & $1,75,7$ & 1,648 & 2,505 & 4.252 & 4.825 & 4,202 \\
\hline Nevalda & 8901 & 1,004 & 1,100 & 1,533 & 2.125 & 2.156 \\
\hline New Hampshire & 188 & 339 & 551 & 771 & 753 & 842 \\
\hline New Jersey ...... & 6,0837 & 7,5637 & 10,928 & 15,624 & 15,506 & 16,826 \\
\hline New Mrexico & 1.211 & 1.517 & 2,050 & 3,377 & 4,499 & 5,242 \\
\hline Now York & 8,747 & 12,079 & 18,494 & 25,314 & 24.630 & 26,565 \\
\hline ivorth Ciarolina ................... & 1.568 & 2,022 & 3,046 & 1,367 & 4,016 & 4,784 \\
\hline North l.akota & 335 & $50 \theta$ & 1,027 & 1,511 & 1,669 & 1,789 \\
\hline$\quad . \quad$. & 3.927 & 8,237 & 13,360 & 21,1837 & 24,435 & 29,636 \\
\hline Oklahorna & 1.278 & 1.600 & 2,867 & 6,195 & 7,031 & 6,067 \\
\hline Oregon .. & 989 & 1,140 & 1.724 & 2,761 & 3,283 & $2.8 B A$ \\
\hline Pennsylvana & 4,649 & 8.264 & 11.996 & 16,9833 & 18,693 & $19,32 ?$ \\
\hline Rhorde litand & 457 & 565 & 855 & 1,003 & 979 & 1,032 \\
\hline South Carolina & 8991 & 1.094 & 1.489 & 2,096 & 1,936 & 2,176 \\
\hline South Datkola & 276 & 413 & 776 & 1,253 & 1.454 & 1,339 \\
\hline Tenressue & 1.918 & $2,6,32$ & $4,05,9$ & 6,390 & 6,849 & 6,960 \\
\hline Texas ........ & 10,829 & 11.404 & 15.881 & $20,2.27$ & 23,0834 & 21.405 \\
\hline Ulah $\ldots \ldots$ & $47 ?$ & 663 & 1,227 & 2,2830 & 3,376 & 3,283 \\
\hline 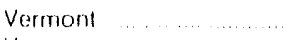 & 66 & 105 & 199 & 283 & 300 & 315 \\
\hline Virgiria & 2.129 & 2,446 & $4,0 \leq 1$ & 5.721 & 6,056 & 6,164 \\
\hline Wasthmyton & 1.851 & 2,194 & 3.672 & 6.424 & $\{, 56\} \mid$ & 5,343 \\
\hline Wost Virgiria & $8 B 1$ & 1.476 & 2.191 & 2.8307 & 3,260 & 3,177 \\
\hline Wisconisin & 2.012 & $3.88 ?$ & $6,66 ?$ & 10,1683 & 10.9887 & 10.505 \\
\hline Wyorruriy & 3683 & 521 & $86 \%$ & 1,111 & 1,114 & 1.367 \\
\hline Tolat & 120.901 & 158,8445 & $233,4,08$ & $341,5,63$ & 379,8999 & 376,073 \\
\hline
\end{tabular}

H Rrevised loatis

Noles and Sounces Sise the lats page of thes sectors 
Table 24. Natural Gas Deliveries to Industrial Consumers by State (Million Cubic Feet)

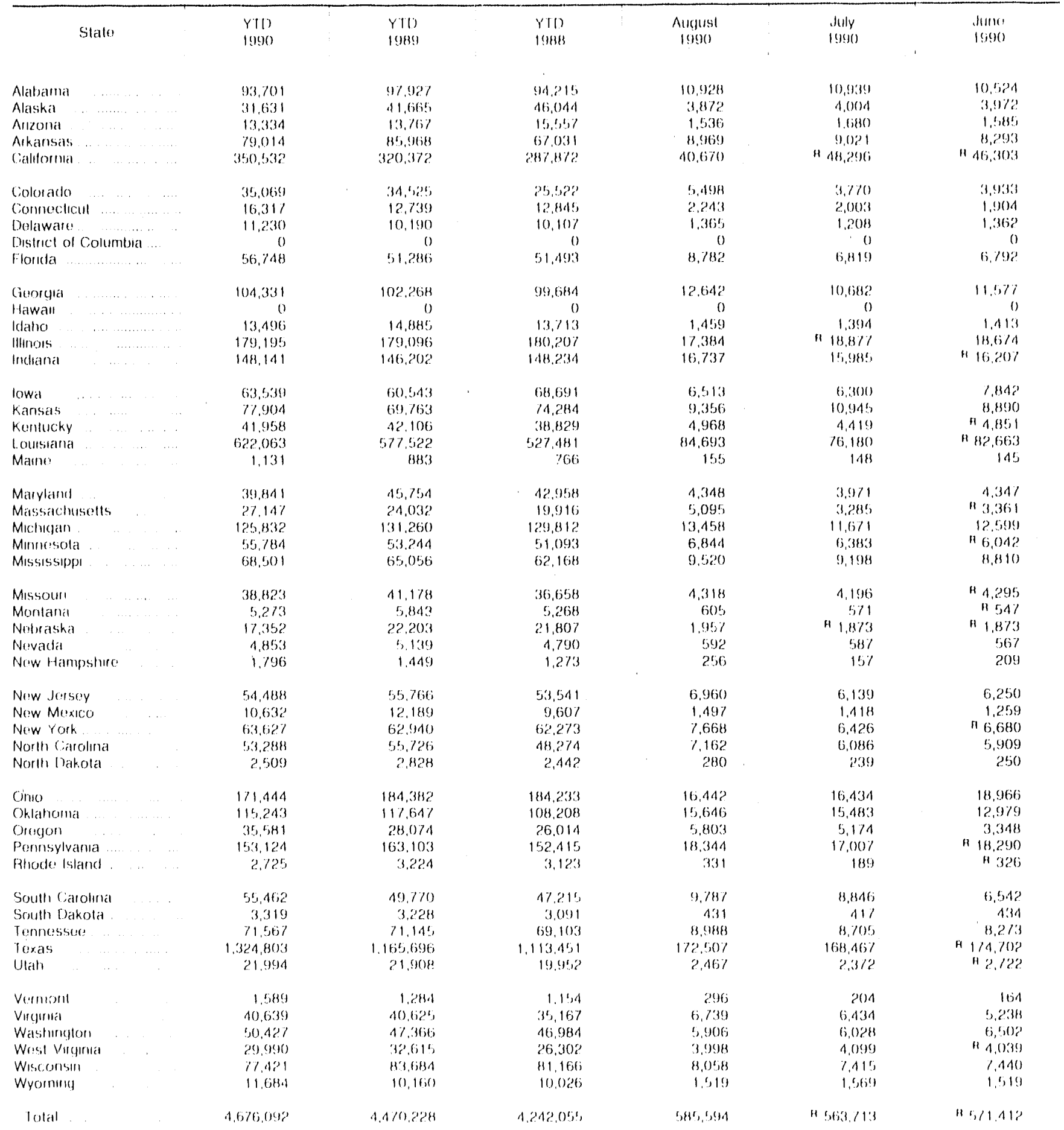

Sere formotes at end ol lathe 
Table 24. Natural Gas Deliveries to Industrial Consumers by State (Continued) (Million Cubic Feet)

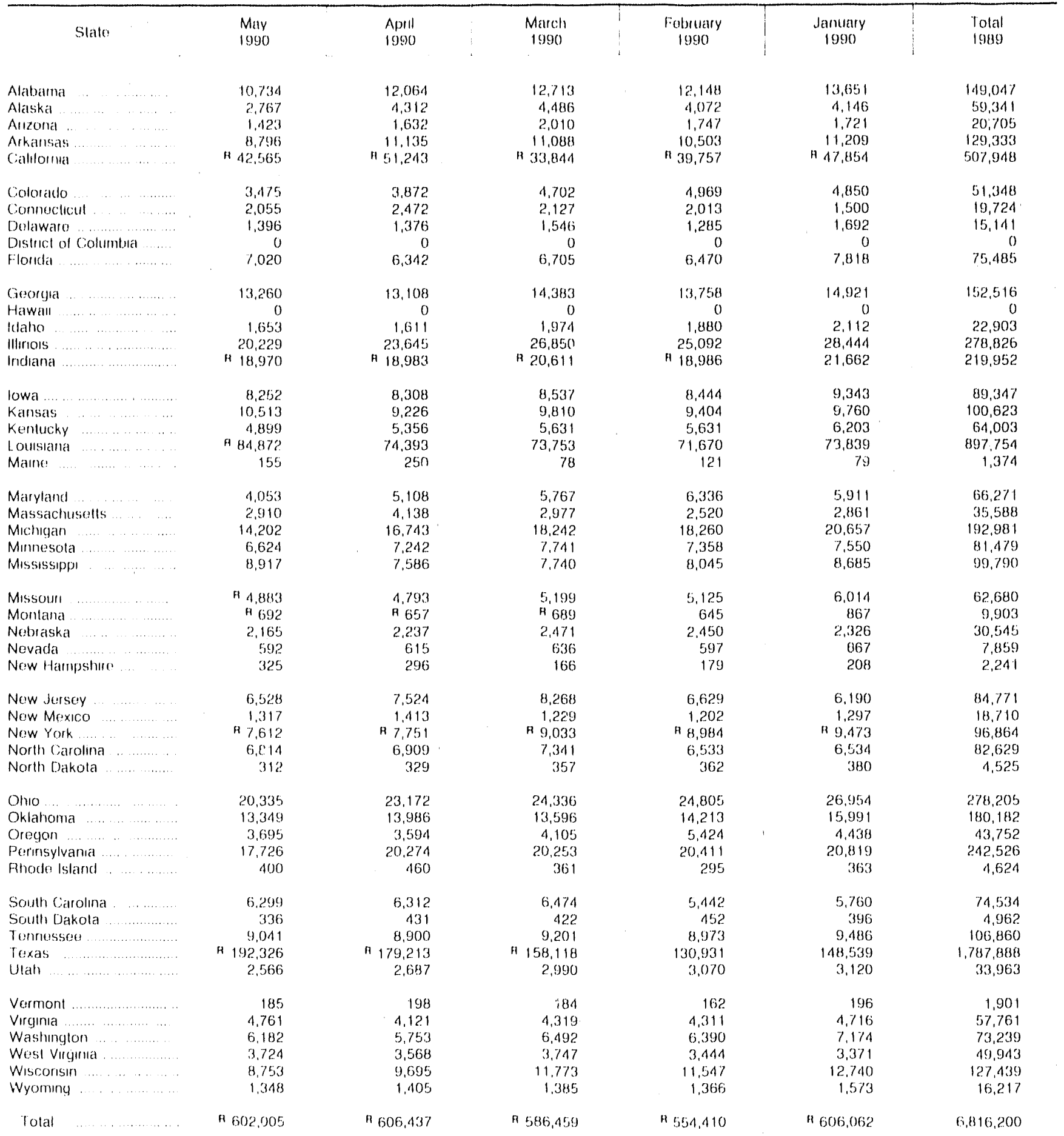

See foolnotes at end of lable 
Table 24. Natural Gas Deliveries to Industrial Consumers by State (Continued) (Million Cubic Feet)

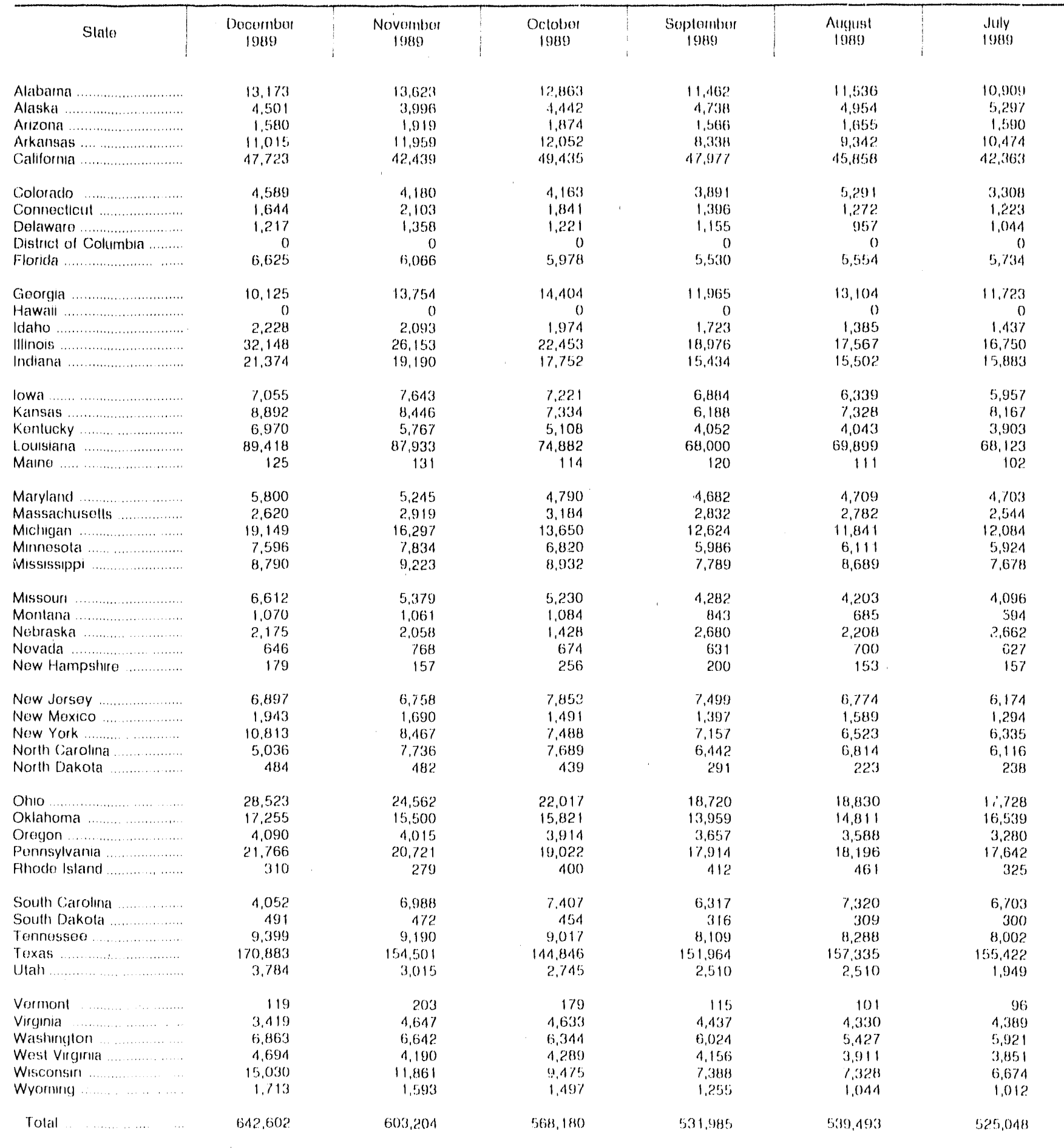

See lootnotos at ond of lable 
Table 24. Natural Gas Deliveries to Industrial Consumers by State (Continued) (Million Cubic Feet)

\begin{tabular}{|c|c|c|c|c|c|c|}
\hline Slate & $\begin{array}{l}\text { Juno } \\
\text { l(1)8!) }\end{array}$ & $\begin{array}{l}\text { Maly } \\
\text { 1980 }\end{array}$ & $\begin{array}{l}\text { April } \\
1080\end{array}$ & $\begin{array}{c}\text { March } \\
1989\end{array}$ & $\begin{array}{l}\text { Fobmuary } \\
1049\end{array}$ & $\begin{array}{l}\text { January } \\
\text { lgseg }\end{array}$ \\
\hline 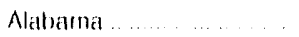 & 11,843 & 12,378 & 12.704 & 13,3418 & 12,184 & 13,025 \\
\hline 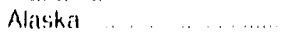 & 5,573 & 5,123 & 5,517 & 5,618 & $\quad 1,671$ & 1,912 \\
\hline Arrzonar & 1.753 & 1,8837 & 1,613 & 1,6831 & 1,613 & 1,075 \\
\hline Arkanlsals & 9,663 & 0,751 & 11,392 & $13,4 \% 2$ & 11,511 & 10,411 \\
\hline 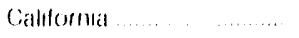 & 41,341 & 12.425 & $34,4,34$ & 42,904 & $28,32 ?$ & 42,725 \\
\hline 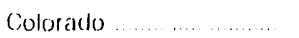 & 3,456 & $3,93 ?$ & 4,517 & 4,657 & $1,79 n$ & 4,570 \\
\hline Connocticul & 1,480 & 1,610 & 1,927 & 1.929 & 1,641 & 1.6577 \\
\hline 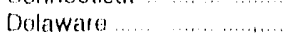 & 1,332 & 1,581 & 1,647 & $1,2.18$ & $\begin{array}{l}1,071 \\
1,230\end{array}$ & 1.181 \\
\hline Distret of Columbia........ & 0 & 0 & 0 & $\begin{array}{r}1.610 \\
0\end{array}$ & 1,60 & 0 \\
\hline Flonda $\ldots$.............. & 6,021 & 6,725 & 6,379 & 7,003 & 6,746 & 7,124 \\
\hline Goorgla & 12,573 & 12,878 & 13,035 & 14,086 & $11,82.4$ & 13,045 \\
\hline (n) & 0 & 0 & $\begin{array}{r}10,000 \\
0\end{array}$ & 0 & 0 & 0 \\
\hline Idaho & 1,779 & 2,024 & 1,995 & 2.127 & 1,973 & 2,165 \\
\hline Illinols & 16,046 & 20,708 & 22,567 & $2.7,7506$ & 29,869 & 27,831 \\
\hline 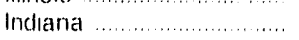 & 16,315 & 18,391 & 18,725 & 21,073 & 20,547 & 19,766 \\
\hline lowa & 6.757 & 6,880 & 7,837 & 8,850 & 8,943 & 8,980 \\
\hline 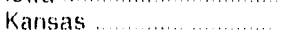 & 7904 & 8,458 & 8,920 & 10,005 & 8,978 & 0,994 \\
\hline Kontucky ...................... & 4,596 & 4,929 & 5,309 & 6.273 & 6,485 & 6,568 \\
\hline Loulsiana & 70.236 & 72,135 & 70,526 & 75.115 & 73,908 & $7 \%, 190$ \\
\hline 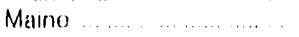 & 110 & 130 & 130 & 101 & 104 & 95 \\
\hline 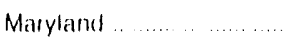 & 5,102 & 5,333 & 6.106 & 6.932 & 6.527 & 6,342 \\
\hline Massachusolls ........... & 2,933 & 3,714 & 3,350 & 3,429 & 2,5983 & 2,682 \\
\hline Michlgan ...................... & 13,412 & 14,840 & 17,990 & 20,718 & 199949 & 20,387 \\
\hline Minnessola ... ............... & 5,5419 & 5,857 & 6,607 & $8,1,17$ & $7,32.9$ & 7,720 \\
\hline Mississipp! $\ldots \ldots \ldots$ & 8.323 & 8,080 & 8,487 & 7,083 & 7,554 & $4,16 ?$ \\
\hline 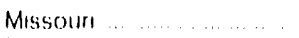 & 4,321 & 4.759 & 5,3193 & 6,140 & 6.328 & 5,0338 \\
\hline 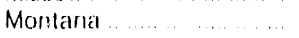 & 658 & 678 & 756 & 766 & 756 & 950 \\
\hline Nobraska & 2,574 & 2.754 & 2,721 & 3,201 & 3,001 & $3,08 ?$ \\
\hline Novada ......................... & 625 & 647 & 649 & 623 & $582^{3}$ & 686 \\
\hline Now Hampshure ....... & 1984 & 239 & 259 & 141 & $|A|$ & 158 \\
\hline Now Jfisey ................ & 6,906 & 7,213 & $7, \pi 99$ & $8,04 t$ & 6,167 & 6,898 \\
\hline Now Mexico ................ & 1,313 & 1.765 & 1,527 & 1,303 & 1,187 & 1,011 \\
\hline 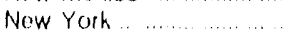 & 6,930 & 6,790 & 8,376 & 9,782 & 8,752 & 9,152 \\
\hline North Carolma ........... & 6,612 ? & 7,290 & 7,624 & 7,217 & 0,583 & 7,256 \\
\hline North Dakola ............. & 305 & 362 & 383 & 426 & 403 & 488 \\
\hline Oho $\ldots \ldots \ldots \ldots$ & 19,118 & 21,669 & 25,018 & 27,485 & $27,0,31$ & 27,500 \\
\hline Oklahoma & 14,439 & 13.217 & 14,003 & 15,565 & 14,199 & 11,874 \\
\hline 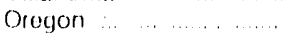 & 3,102 & 3,501 & 3,490 & 4,052 & 3,268 & 3.793 \\
\hline Ponnsylvania & 18,101 & 20.570 & 20,953 & 23,120 & 22,3633 & 22,158 \\
\hline Rhode Island ............. & 440 & 515 & $46 ?$ & 351 & 325 & 345 \\
\hline Soulh Ciarolma & 7.174 & 6,823 & 6.577 & 6,194 & 1,668 & 4,311 \\
\hline South Dakota & 363 & 373 & 118 & 447 & 493 & 525 \\
\hline 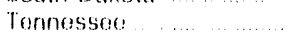 & 8.403 & 7,9333 & 9,304 & 9,354 & 9,728 & 10,133 \\
\hline Toxas .... & 147.747 & 140.214 & 144,112 & 139,485 & 139,004 & 142,377 \\
\hline 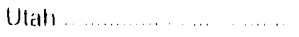 & 2,599 & 2,518 & 2.718 & 3,088 & 3.153 & 3,373 \\
\hline 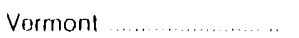 & 122 & 140 & 187 & 211 & 193 & 2.34 \\
\hline 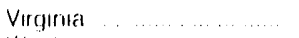 & $5,13 ?$ & $4,83 \%$ & 6.248 & 5,474 & 1,980 & 5,240 \\
\hline Washmeglon $\ldots . .$. & 5,357 & 5,834 & $5,96 ?$ & 6,494 & 5,902 & 6,370 \\
\hline Wost virçina & 3,584 & 4,038 & 4.134 & 4.053 & 4,339 & 4,706 \\
\hline Wisconsint & 7.528 & 83,609 & 10,971 & 13,630 & 14,915 & 14,020 \\
\hline Wyoummy _........... & 1.107 & 1.254 & $1,2.38$ & 1,1774 & 1,448 & 1,683 \\
\hline 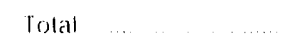 & 529,173 & $544,31 \%$ & 562.716 & 601,5003 & 569,696 & 599,2833 \\
\hline
\end{tabular}

h Rovisegl l anta

Notos and Sourcess Sue the last page of this socton 
Table 25. Natural Gas Deliveries to Electrical Utility Consumers by State

(Million Cubic Feet)

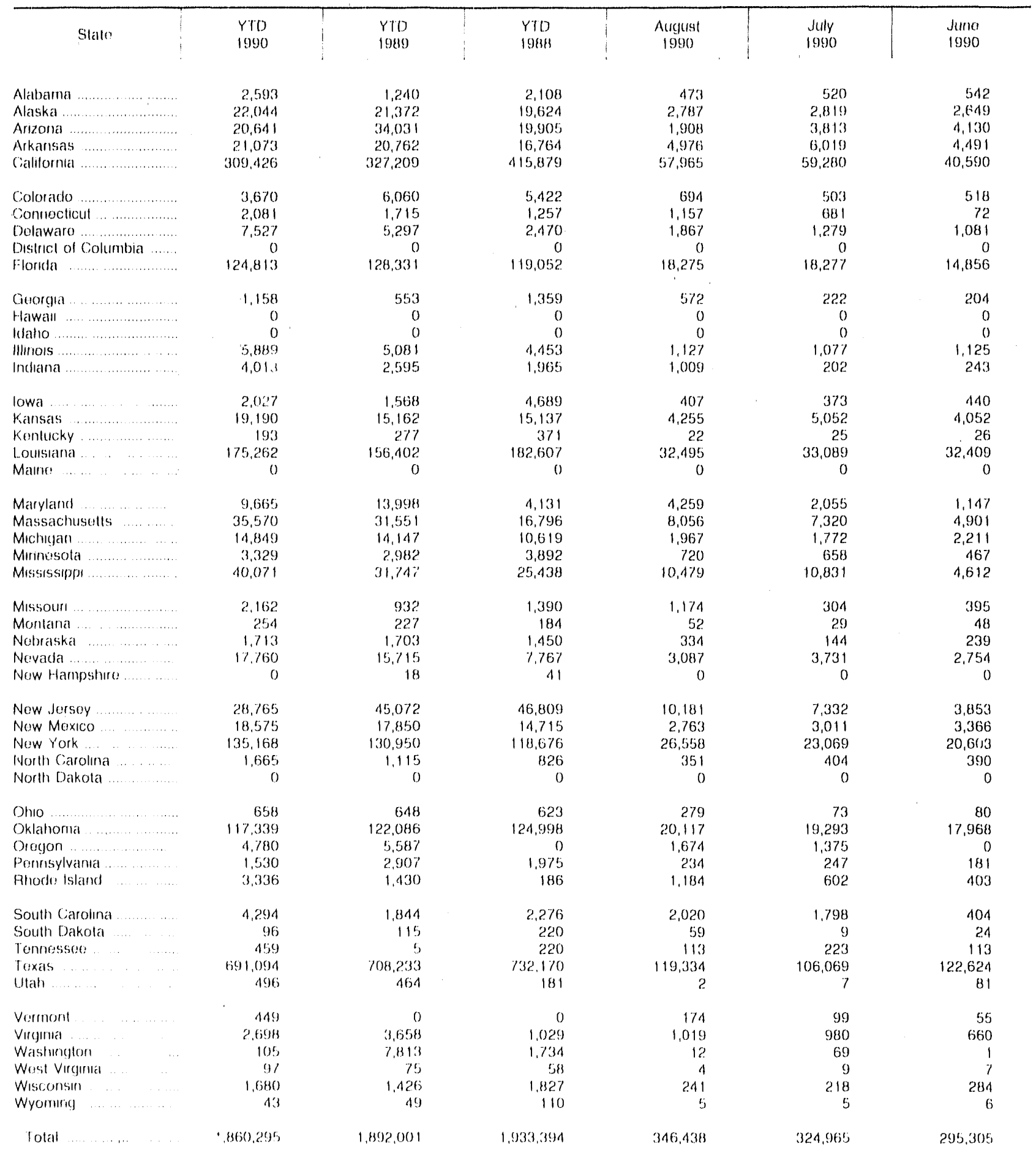

Ses lostnotre; at rend of lable 
Table 25. Natural Gas Deliveries to Electrical Utility ${ }^{a}$ Consumers by State (Continued)

(Million Cubic Feet)

\begin{tabular}{|c|c|c|c|c|c|c|}
\hline Slatu: & $\begin{array}{l}\text { Mily } \\
1990\end{array}$ & $\begin{array}{l}\text { Apsill } \\
1 !(900\end{array}$ & $\begin{array}{l}\text { Matrch } \\
1590)\end{array}$ & $\begin{array}{c}\text { folmbaty } \\
1990\end{array}$ & $\begin{array}{l}\text { January } \\
19900\end{array}$ & $\begin{array}{l}\text { lolal } \\
\text { logse }\end{array}$ \\
\hline Alabama & $31 B$ & 288 & 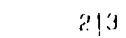 & $1: 30$ & 100 & 1,707 \\
\hline Alalskal & 2.610 & 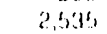 & 2.860 & 2,920 & 2.904 & $3 \%, 7196$ \\
\hline Anzona & $\therefore 6833$ & $2.09 \%$ & 2.219 & 1,974 & $1,96 \%$ & $50,80) \%$ \\
\hline Arkansals & $\therefore 61 \%$ & 745 & (i) & 973 & bido & $20,462 ?$ \\
\hline Cablitorna & 31,8230 & 34,310 & 31,$46 ; 3$ & 24.795 & 29.203 & $51 i, 700$ \\
\hline Colorado & $46 !$ & 375 & 499 & 258 & 364 & 8,376 \\
\hline Conneticul & 1688 & 0 & 1 & 1 & 1 & 3.291 \\
\hline Delawaro & $6(3)$ & 583 & 927 & (110) & 351 & 7990 \\
\hline Desitret of Columbia & 0 & 0 & 0 & 0 & () & () \\
\hline Flonda & 17.56 .7 & 16.239 & 15,353 & 12.531 & 11.225 & 186,8314 \\
\hline Cirorgla & 74 & 34 & 29 & $1 \%$ & 11 & tiss4 \\
\hline Hawall & 0 & 0 & 0 & () & 0 & 0 \\
\hline Idatho ... & 0 & 0 & 0 & 0 & 0 & () \\
\hline Illinous & 6881 & $50 \theta$ & $5,4 \%$ & $40 \%$ & 417 & 6,967 \\
\hline Indianat & 639 & 561 & 499 & 4:3:3 & 4:3? & $A, 0)$ \\
\hline Iowa & 301 & $1 \% 6$ & 1334 & 101 & 91 & $2,40 \%$ \\
\hline Kansas & 2,071 & 926 & 1,121 & $\{1 \leqslant\}$ & 1305 & 18.741 \\
\hline Konlucky & 12 & 26 & 36 & $3{ }^{\prime}$ & 11 & 328 \\
\hline I.ousiana & 21,148 & 16.432 & 16,420 & 11,415 & 11.554 & $227,08 \%$ \\
\hline Manne $\quad \therefore \ldots$ & 0 & 0 & 0 & 0 & 0 & 0 \\
\hline Maryland ... . . . & 556 & $69 ?$ & $59 \%$ & 163 & 201 & 19,1884 \\
\hline Massachuselts; & 7,8319 & 5,734 & 1,166 & 5,57 & 183 & 48,4148 \\
\hline Michugan ........ & 1.718 & 1.185 & 2.118 & 1,$65 ; 0$ & 2.2283 & 18.782 \\
\hline Minnosola & 376 & 311 & :148 & 206 & 243 & 4.321 \\
\hline Mississippl _.. & 3.585 & 33,331 & 3,071 & 2.016 & 2.146 & $44,9 ? 7$ \\
\hline Missount & 83 & $5 \%$ & 61 & $4: 3$ & 50) & 1.242 \\
\hline Monlana & 27 & 31 & 22 & 10 & 26 & 336 \\
\hline Notraska & 370 & 336 & 185 & 16 & 50 & 2.503 \\
\hline Nevarda & 2.458 & 2.117 & 1.509 & 823 & 1.186 & 23,210 \\
\hline Now Hampsihure & 0 & 0 & 0 & () & () & 23 \\
\hline Now Jersisy & 1.594 & 3.273 & 1.631 & 686 & 215 & $54,7 / 8$ \\
\hline$N(: w$ Muxico & 2,751 & 2.213 & 1,931 & 997 & 1.543 & 27.365 \\
\hline New York & 18,466 & $17.05,4$ & 17,193 & $6,97.3$ & $5,25 ?$ & 182,000 \\
\hline North Carolura ... .. & 163 & 109 & $10 ?$ & 76 & 70 & 1.673 \\
\hline North Dakota & 0 & 0 & 0 & 0 & 0 & 1 \\
\hline$\ldots$. & 49 & 13 & 31 & 30 & 40 & . $\quad 333$ \\
\hline Oklahoma & $13,62,4$ & 13.146 & $11.00 \%$ & 10,5889 & 11.609 & $1780 \% 1$ \\
\hline Orengon & 0 & 0 & 0 & 1,496 & 2,35 & 12,19 \\
\hline Punnigylvantial. & $15 f_{i}$ & 20 & 161 & 156 & 173 & 4.0?? \\
\hline Hhorde Island & $62 ?$ & $40 \mathrm{H}$ & $B S$ & 0 & 35 & 2.147 \\
\hline South Cirrolien & $2 \beta$ & $1 ; 3$ & 12 & 11 & Bs & 2,704 \\
\hline South rrincta & 1 & 1 & 1 & 0 & 1 & 132 \\
\hline lomiesser: & 0 & 0 & 10 & 0 & () & 18 \\
\hline Toxas & $100.46 ; 3$ & 11.271 & Grista & 96.978 & 57.433 & $1,023,703$ \\
\hline Wih .... & 122 & 130 & 153 & 0 & 1 & tiski \\
\hline Verrirmt & 49 & 46 & 3 & $?$ & 21 & 31 \\
\hline Vurguma & 26 & 10 & () & (1) & 1 & 3.196 \\
\hline Washongton & !) & 0 & $?$ & 4 & 3 & $3,3 ?()$ \\
\hline Wrest Virclunat & 10 & If & 2 & 18 & 31 & 101 \\
\hline Wisconsin & 280 & 281 & $17 \%$ & 101 & yHs & $3(1) / 6$ \\
\hline Wyenmence & 7 & ; & is & 5) & s & H! \\
\hline Tolal & 239.415 & $196 ; 8330$ & 183.456 & 131,273 & $14: 3,6344$ & $\therefore .76 \% .003$ \\
\hline
\end{tabular}

Sren tootnoles at and of latele: 
Table 25. Natural Gas Deliveries to Electrical Utilitya Consumers by State (Continued)

(Million Cubic Feet)

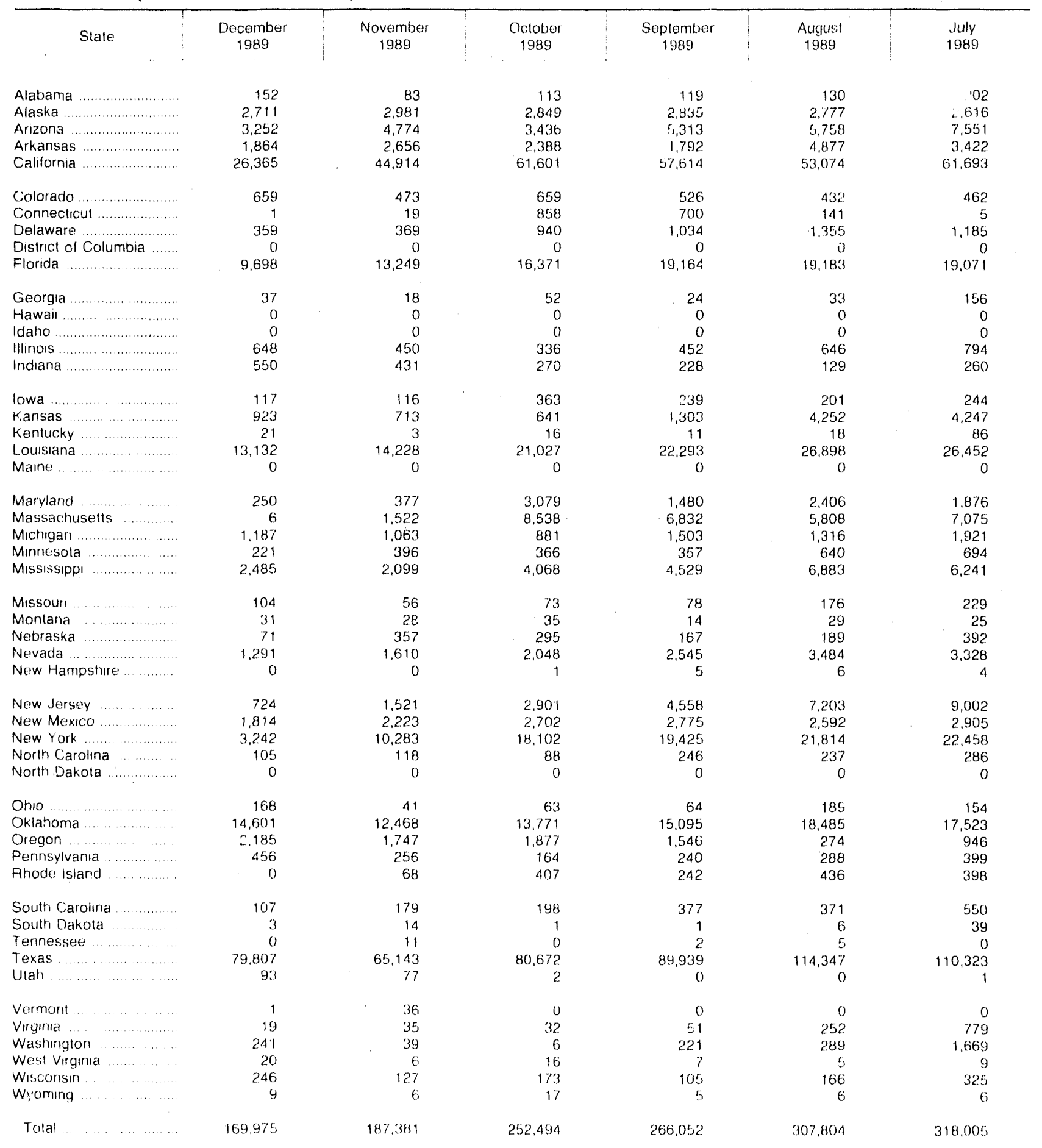

See footriotes at end of table 


\section{Table 25. Natural Gas Deliveries to Electrical Utility ${ }^{a}$ Consumers by State (Continued)}

(Million Cubic Feet)

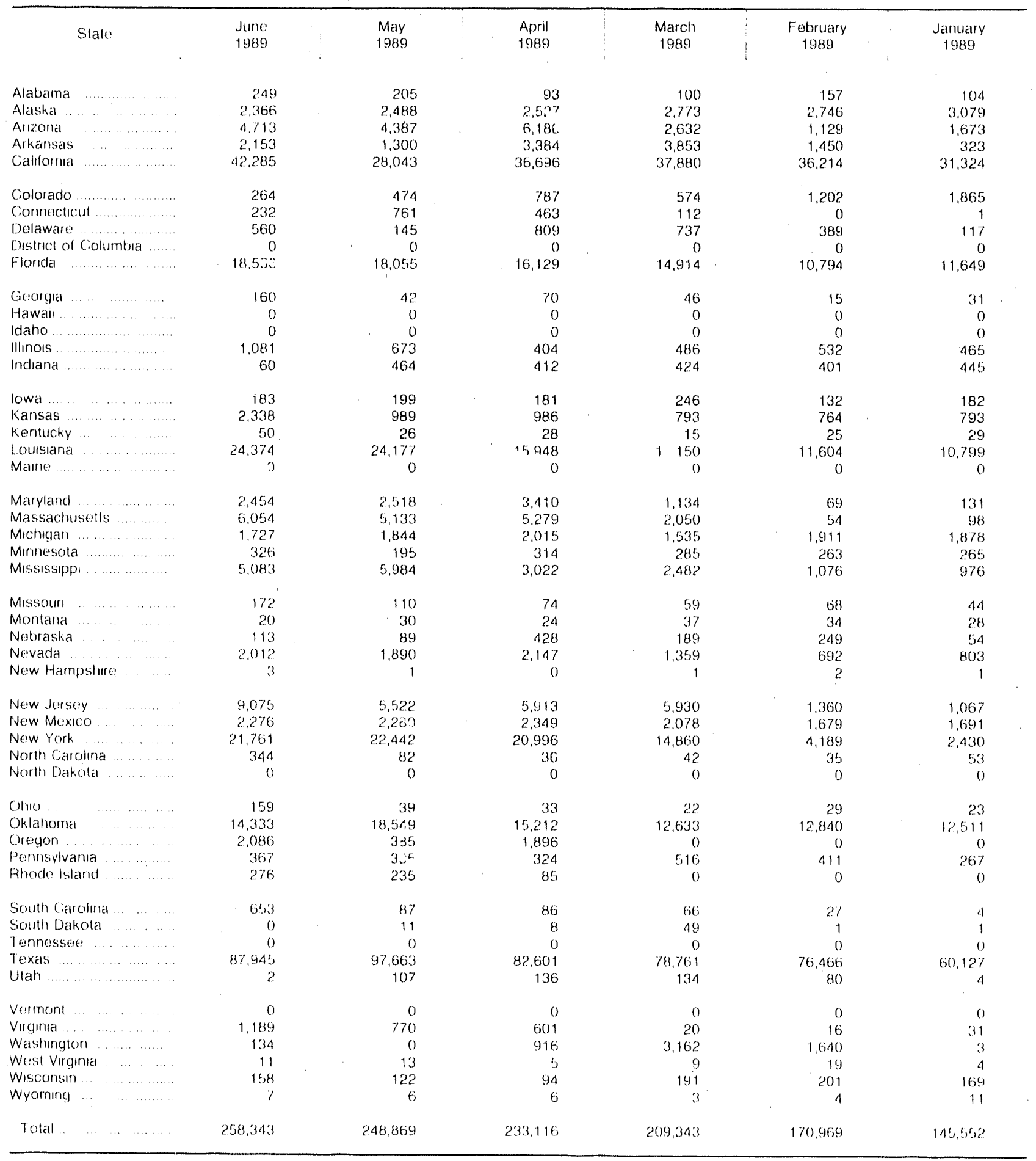

"Includes all steam electric; utilly generating plants with a combined capacity of 50 meqawatts or greater Noles and Sources. Sere the lasit page of this section 
Table 26. Natural Gas Deliveries to All Consumers by State (Million Cubic Feet)

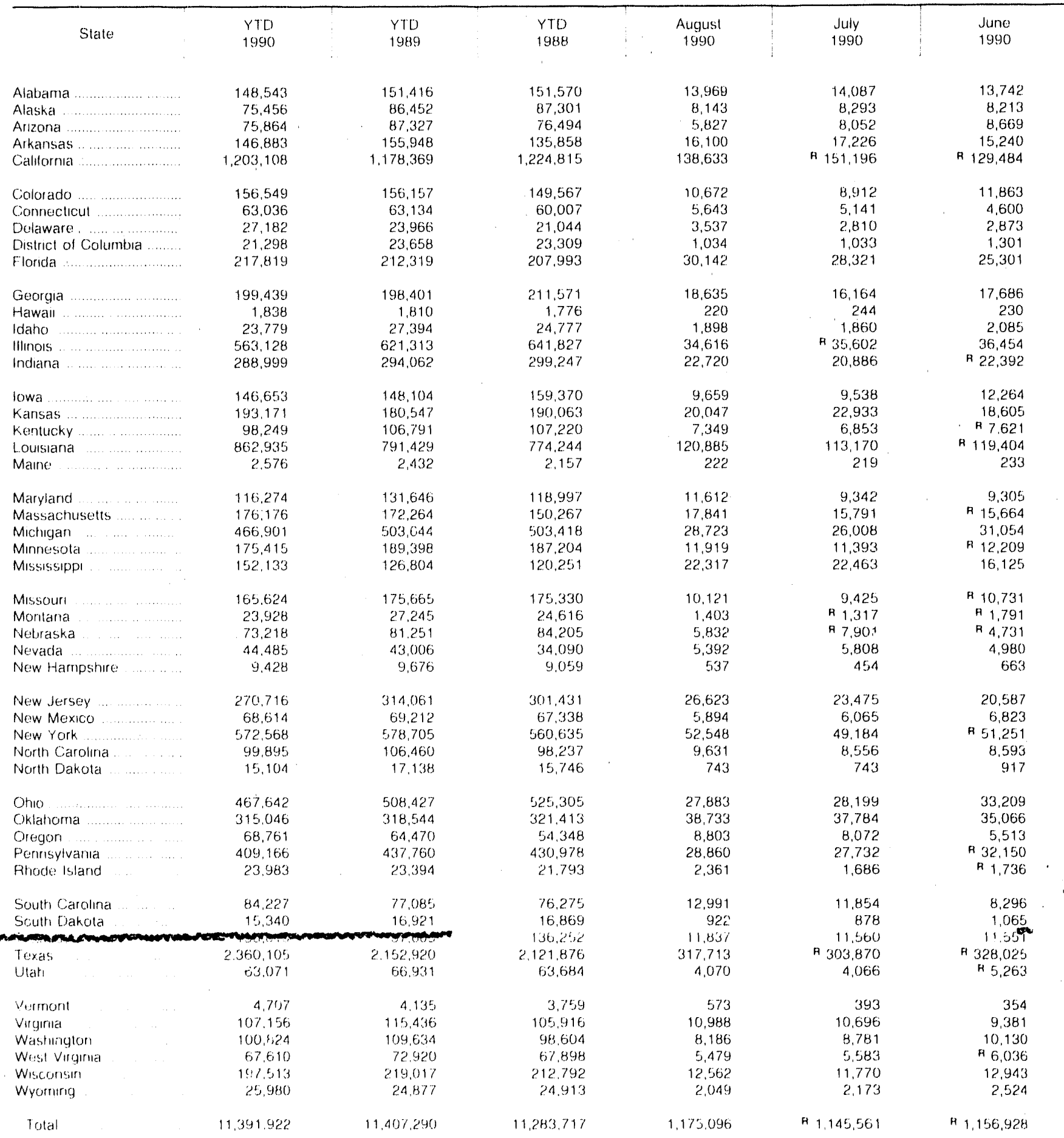

Seo tortnotes at end of tables, 
Table 26. Natural Gas Deliveries to All Consumers by State (Continued) (Million Cubic Feet)

\begin{tabular}{|c|c|c|c|c|c|c|}
\hline - State & $\begin{array}{l}\text { May } \\
1990\end{array}$ & $\begin{array}{l}\text { April } \\
1990\end{array}$ & $\begin{array}{l}\text { Match } \\
1990\end{array}$ & $\begin{array}{c}\text { February } \\
1990\end{array}$ & $\begin{array}{c}\text { January } \\
1990\end{array}$ & $\begin{array}{l}\text { Total } \\
1989\end{array}$ \\
\hline Alabáma & 15,431 & 18,804 & 20,992 & 22,008 & 29,510 & 225.124 \\
\hline Alaska & 7.443 & 9.669 & 11.294 & 11,396 & 11,005 & 127,414 \\
\hline Arrzona & 7.823 & 8.217 & 11,336 & 12,737 & 13,201 & 127,177 \\
\hline Alkansas & 15,004 & 17.976 & 19.217 & 20.492 & 25,628 & 228.392 \\
\hline Cahlorma & A 124,015 & ค 146,725 & H 153,355 & A 173.197 & A 186,503 & $1.799,042$ \\
\hline Colorado & 16.475 & 21,249 & 26,753 & 28,646 & 31.979 & 218.766 \\
\hline Comnecticut & 5,934 & 8.758 & 10,146 & 10,969 & 11,845 & 94,484 \\
\hline Delaware $\ldots$ & 2.757 & 3,103 & 3,996 & 3.688 & 4,418 & 34.919 \\
\hline District of Columbia & 1.809 & 2,928 & 3,801 & 4,084 & 5,308 & 33,174 \\
\hline 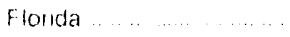 & 28.557 & 27,047 & 27,004 & 24,248 & 27,199 & 310,493 \\
\hline Georgla & 20,254 & 23,939 & 30,243 & 32,224 & 40,294 & 309,970 \\
\hline Hawall & 220 & 223 & 236 & 233 & 232 & 2,694 \\
\hline$\ldots$ & 2,507 & 2.721 & 3,832 & 4.297 & 4,579 & 40,679 \\
\hline$\ldots \ldots$ & 51,721 & 75.155 & 96,004 & 109.304 & 124.272 & 978.413 \\
\hline Indiana $\ldots$ & R 29.847 & R 38.920 & F 46.290 & R 48,949 & 58,995 & 453,526 \\
\hline$\ldots \ldots$ & 15,022 & 19,884 & 23,734 & 25.289 & 31.263 & 215.294 \\
\hline Kansas & 20.564 & 22.971 & 25,637 & 27.982 & 34.432 & 253.952 \\
\hline kentucky & 8,891 & 12.994 & 15,390 & 18,158 & 20,993 & 165,562 \\
\hline Louisıatha & H 111,392 & 98.450 & 99,973 & 94.119 & 105,542 & $1,209,158$ \\
\hline Maife: & 293 & 464 & 336 & 397 & 412 & $3,67 \%$ \\
\hline Maryland ...... & $y, 75,1$ & 15,199 & 18,292 & 18,667 & 24,106 & 187.512 \\
\hline Massachusetts . & 20,964 & 26.204 & 24,737 & 24,645 & 30,330 & 247.205 \\
\hline Michman & 43.535 & 63.445 & 82,547 & 88.529 & 103,060 & 749.612 \\
\hline Minnesota & 16.009 & 22.877 & 30,168 & 33.342 & 37,498 & 287.891 \\
\hline Mississippi & 15.981 & 16,271 & 17.189 & 17,658 & 24,129 & 188.597 \\
\hline Missouri & H 14.453 & 22,216 & 26.388 & 29,842 & 42,448 & 256,105 \\
\hline Montana $\ldots$ & ค 2.602 & म $3,16.1$ & A 4,037 & 4,502 & 5,113 & 41.575 \\
\hline Netraska & 6.303 & 9.173 & 11.297 & 13.009 & $14,77 ?$ & 115,293 \\
\hline Nevada .... & 4.824 &, 924 & 5.958 & 6.097 & 6.502 & 62.950 \\
\hline New Hampshire & 986 & $: 398$ & 1,514 & 1.771 & 2,105 & 13,925 \\
\hline New Jersey & 23.443 & 37,630 & 44,060 & 42.727 & 52,171 & 452,476 \\
\hline New Mexico & $i .364$ & 8,517 & 10,353 & 10,968 & 12,630 & 101,126 \\
\hline New rork. & A 57.440 & R 77.692 & A 96.271 & A 90.758 & म.96,924 & 839,957 \\
\hline North Carolina & 10.222 & 12.819 & 14.989 & 14.939 & 20,146 & 156,105 \\
\hline INorth Dakola & 1.420 & 2,178 & 2.597 & 3,045 & 3,461 & 24.959 \\
\hline Otho & 44.159 & 63,963 & 81.615 & 89,597 & 99017 & $799.85 ?$ \\
\hline Oklahoma & 33,875 & 37,097 & 39,049 & 42.102 & 51,340 & 468,488 \\
\hline Oregon & 6.211 & 6.683 & 9.407 & 13,171 & 10,901 & 99,448 \\
\hline Pennsylvana & 37.739 & 57,116 & 67.026 & $70,79 ?$ & 87,751 & 649,804 \\
\hline Phode Island & 2.757 & 3.491 & 3,671 & 3,605 & 4.676 & 33,821 \\
\hline South Cearolna & 8,194 & 9.234 & 10,456 & 9.846 & 13,352 & 114,237 \\
\hline South Dakota & 1.363 & 2.058 & 2.639 & 3,045 & 3,370 & 25,263 \\
\hline Teroletses & whese & $1 t, i n b$ & 18,449 & 20.782 & 28.8899 & 203.588 \\
\hline Texas & A 323.834 & A 289.548 & ค 278,566 & 232,032 & 286,117 & $3.225,961$ \\
\hline Utah & 6.030 & 7.194 & 10.936 & 12,582 & 12.930 & 96.284 \\
\hline Vermont & $45, i$ & ti20 & 704 & 720 & 887 & 6,145 \\
\hline Virgiria & $9.49 ?$ & 13.085 & 15.940 & 16.400 & 21.174 & 167.450 \\
\hline Wastungton & 11.129 & 11.900 & 15,628 & 17,596 & 17.474 & 158,420 \\
\hline Wesi Virgina & 6,674 & 8,981 & 10.223 & 10.7 .39 & 13,895 & 110.452 \\
\hline Wisconsin & 19,102 & 25,091 & 34.954 & 37.755 & 43,336 & 326,613 \\
\hline Wvommy & 2.840 & 3.385 & 3,997 & 4.278 & 4.734 & 36,633 \\
\hline Total & R $1,249,073$ & H $1,440,735$ & н $1.623,726$ & R 1.657 .955 & $\mathrm{H} 1.942 .848$ & 17.079 .657 \\
\hline
\end{tabular}

See frotnotes at end of tables 
Table 26. Natural Gas Deliveries to All Consumers by State (Continued) (Million Cubic Feet)

\begin{tabular}{|c|c|c|c|c|c|c|}
\hline State & $\begin{array}{c}\text { December } \\
1839\end{array}$ & $\begin{array}{c}\text { November } \\
1989\end{array}$ & $\begin{array}{c}\text { October } \\
1989\end{array}$ & $\begin{array}{c}\text { Seplember } \\
1989\end{array}$ & $\begin{array}{c}\text { August } \\
1989\end{array}$ & $\begin{array}{l}\text { July } \\
1989\end{array}$ \\
\hline 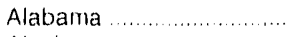 & 24.040 & 19.291 & 16,211 & 14,167 & 14.159 & 14.233 \\
\hline 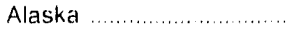 & 11.296 & 10,594 & 9,762 & 9,311 & 9,192 & 9,388 \\
\hline 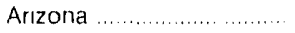 & 11.883 & 10,755 & 7,901 & 9.308 & 9,768 & 11,824 \\
\hline 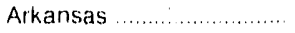 & 23,178 & 19,551 & 17,248 & 12.466 & 16,364 & 16,153 \\
\hline California ............................. & 163,384 & 149,088 & 158,974 & 149,227 & 139,259 & 142,104 \\
\hline 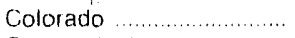 & 24,416 & 17,069 & 11.804 & 9.320 & 10,121 & 8,876 \\
\hline Connecticut ....................... & 12.527 & 7,666 & 6,370 & 4,785 & 3,988 & 3,870 \\
\hline 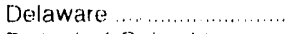 & 3,290 & 2,506 & 2,618 & 2.539 & 2.6334 & 2,549 \\
\hline District of Columbia ......... & 4,689 & 2,189 & 1.430 & 1,20 & 1,246 & 1,199 \\
\hline Florida $\ldots . . . \ldots \ldots \ldots \ldots . . . . . . . . . .$. & 21.767 & $23: 157$ & 25,431 & 27,819 & 27,722 & 28,004 \\
\hline Georgia $\ldots . . . \ldots \ldots \ldots$ & 40,687 & 29.716 & 23,123 & 18,044 & 18.289 & 17,278 \\
\hline Hawall ................................ & 224 & 2.17 & 222 & 220 & 215 & 228 \\
\hline 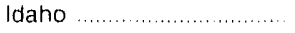 & 4,641 & 3,644 & 2.737 & 2,264 & 1,779 & 1,897 \\
\hline Illınols ............................ & 160,338 & 97,901 & 59.725 & 39,136 & 32,963 & 33,688 \\
\hline Indiana $\ldots . . . \ldots \ldots \ldots \ldots \ldots \ldots \ldots \ldots \ldots$ & 65.183 & 42,379 & 30.067 & 21.834 & 20.158 & 20,615 \\
\hline 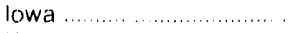 & 26,682 & 17.409 & 12,939 & 10,160 & 9,300 & 9,039 \\
\hline 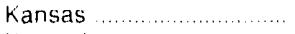 & 28,272 & 18,944 & 13,661 & 12,528 & 18,079 & 17.624 \\
\hline 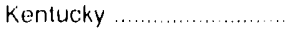 & 26,673 & 15,238 & 10,204 & 6,656 & 6.389 & 6.420 \\
\hline Louisiana ......................... & 115,718 & 108,321 & 99,958 & 93,732 & $100.13 \%$ & 98,136 \\
\hline Maıne ............................... & 490 & 305 & 244 & 202 & 181 & 165 \\
\hline Maryland ........................... & 21,772 & 12,553 & 12.223 & 9,318 & 10,127 & 9.789 \\
\hline Massachusetts …............. & 25,714 & 15,583 & 19.281 & 14,363 & 13.168 & 14,351 \\
\hline Michlgarl ............................ & 108,871 & 64,815 & 43,380 & 28.901 & 25,582 & 26,627 \\
\hline Minnesota ....................... & 41,593 & 28,061 & 17,333 & 11,500 & 11,030 & 11,119 \\
\hline Mississippi ........................... & 18,242 & 14,605 & 14,981 & 13,966 & 17,102 & 15,502 \\
\hline Missouri .......................... & 38.207 & 19.591 & 13,148 & 9.493 & 8,891 & $9.16 ?$ \\
\hline Montana ......................... & 5,400 & 4,052 & 3,010 & 1,867 & 1.530 & 1,375 \\
\hline Nebraska ..................... & 13,739 & 8,805 & 5.804 & 5,693 & 7.645 & 7,513 \\
\hline Nevada .............................. & 5,986 & 5,035 & 4,380 & $4 ; 542$ & 5.467 & 5.276 \\
\hline New Hampshire .............. & 1,922 & 998 & 779 & 549 & 426 & 471 \\
\hline New Jersey ................. & 57.310 & 33,144 & 25,984 & 21,978 & 23.275 & 25,289 \\
\hline New Mexico .................... & 11.333 & 8,329 & 6,283 & 5,970 & 6,062 & 5,941 \\
\hline 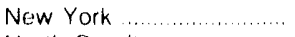 & 98,996 & 63,958 & 53,391 & 44,908 & 45.831 & 47,273 \\
\hline North Carolina .................. & 16.403 & 13,316 & 10,958 & 8,968 & 9,200 & 8,621 \\
\hline North Dakota ....................... & 3,401 & 2,249 & 1,302 & 871 & 626 & 721 \\
\hline Ohio $\ldots \ldots \ldots \ldots \ldots \ldots \ldots \ldots \ldots \ldots \ldots$ & 128,237 & 78,522 & 51,673 & 32.994 & 30,016 & 28,779 \\
\hline 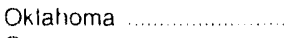 & 48.765 & 35,307 & 33,690 & 32,180 & 36,047 & 37.100 \\
\hline 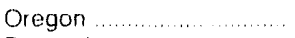 & 11,524 & 9.220 & 7.568 & 6,666 & 5,215 & 5,745 \\
\hline Pennsylvanıa ........................ & 88.193 & 55,008 & 39.594 & 29,248 & 28,658 & 28.714 \\
\hline Ahode Island ....................... & 4,068 & 2,327 & 2,320 & 1.711 & 1,879 & 1,694 \\
\hline South Carolına ................... & 10.035 & 10.016 & 9,195 & 7.906 & 8,827 & 8,556 \\
\hline South Dakota ................. & 3.633 & 2.413 & 1.424 & 870 & 733 & 786 \\
\hline 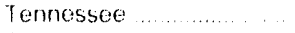 & 26,403 & 16,526 & 13,056 & 11,000 & 10.914 & 10.846 \\
\hline 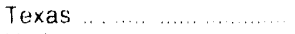 & 318.473 & 249,532 & 2.45 .758 & 269,280 & 290,332 & 286,332 \\
\hline Utah $\ldots \ldots \ldots \ldots \ldots \ldots$ & 12.103 & 0,045 & 4,987 & 4.222 & 4,091 & 3,680 \\
\hline Vermont & 781 & 593 & 406 & 230 & 196 & 193 \\
\hline Vırgına ........................ & 21,385 & 13,093 & 9,689 & 7.847 & 7.407 & $8.51 \%$ \\
\hline Washıngton $\ldots . . . . . .$. & 16.628 & 13,531 & 10,055 & 8.572 & 8.064 & 10,263 \\
\hline West Virginia .... .......... & 14,800 & 9,471 & 7,416 & 5,845 & 5.463 & 5,387 \\
\hline Wisconisin & 47,119 & 28.957 & 19,137 & 12.383 & 1,213 & 11,298 \\
\hline Wyoming. & 4.157 & 3,296 & 2,427 & 1,878 & 1,542 & $1.64 i$ \\
\hline$\ldots \ldots \ldots \ldots \ldots \ldots \ldots$ & 1.993 .577 & $1,426,889$ & 1.201 .260 & $1.050,642 ?$ & $1,078,506$ & $1,081,855$ \\
\hline
\end{tabular}

See footnotes at end of tables; 
Table 26. Natural Gas Deliveries to All Consumers by State (Continued) (Million Cubic Feet)

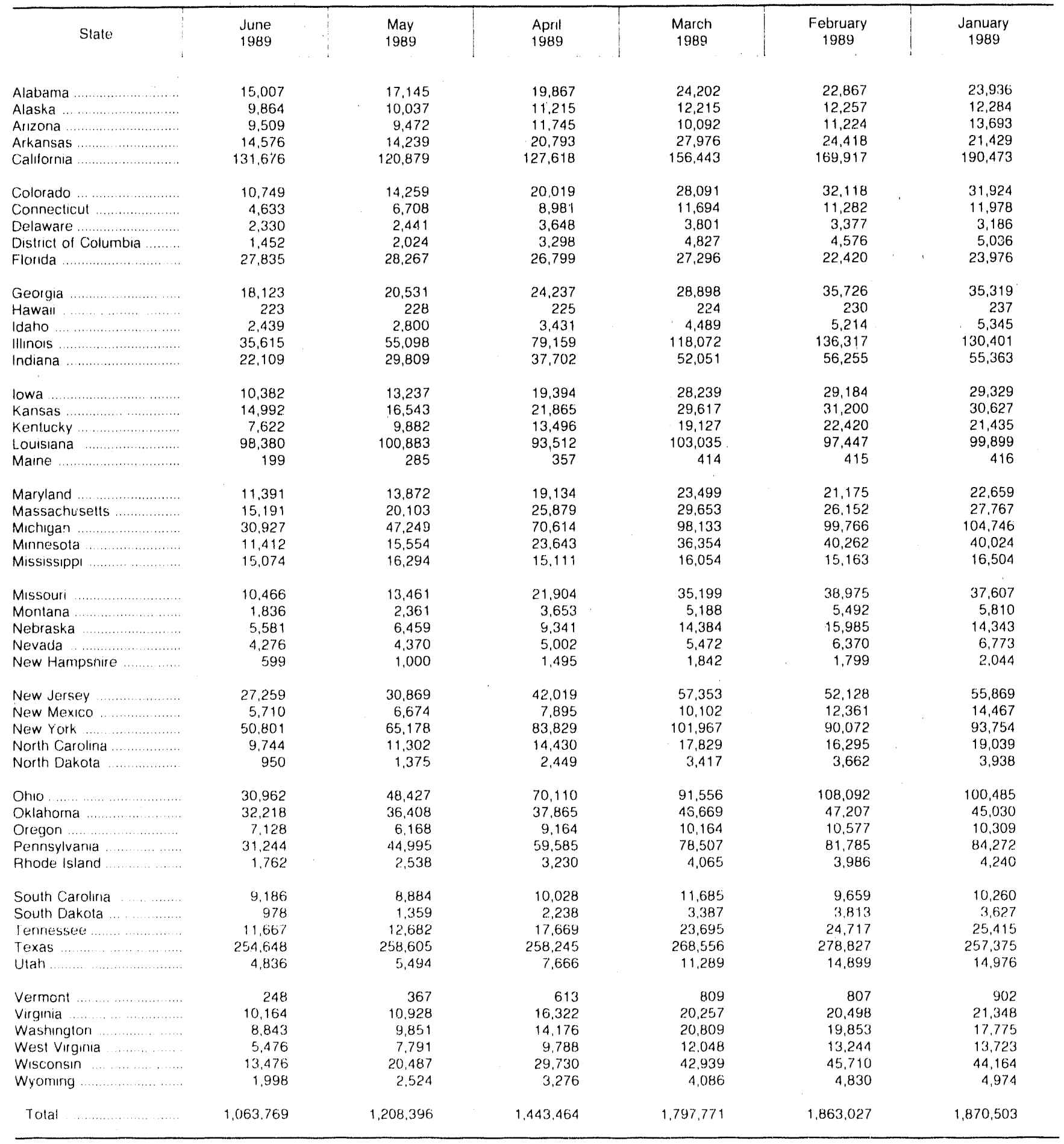

R Revised Data

Notes and Sources: See the last page of this section 
Table 27. Average City Gate Price by State (Dollars per Thousand Cubic Feet)

\begin{tabular}{|c|c|c|c|c|c|c|c|c|}
\hline State & $\begin{array}{l}\text { YTD } \\
1990\end{array}$ & $\begin{array}{l}\text { YTD } \\
1989\end{array}$ & $\begin{array}{l}\text { YTD } \\
1988\end{array}$ & $\begin{array}{c}\text { August } \\
1990\end{array}$ & $\begin{array}{l}\text { July } \\
1990\end{array}$ & $\begin{array}{l}\text { June } \\
1990\end{array}$ & $\begin{array}{l}\text { May } \\
1990\end{array}$ & $\begin{array}{l}\text { April } \\
1990\end{array}$ \\
\hline Alabama ............................ & 3.14 & 2.97 & 3.19 & 3.92 & 2.95 & 3.09 & 2.96 & 2.93 \\
\hline 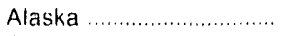 & .33 & .33 & .33 & .35 & .33 & .33 & .33 & 33 \\
\hline 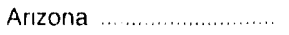 & 2.71 & 2.64 & 2.48 & 2.66 & 2.70 & 2.67 & 2.39 & 2.54 \\
\hline 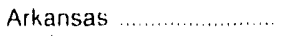 & 2.41 & 2.47 & 2.43 & 2.04 & 2.29 & 2.34 & 2.34 & 2.36 \\
\hline California .......................... & 2.89 & 2.74 & 2.53 & 2.87 & 3.23 & 2.90 & 2.83 & 2.79 \\
\hline 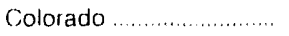 & 2.95 & 3.04 & 3.08 & 3.39 & 3.40 & 3.46 & 3.19 & 2.88 \\
\hline Connecticut .................... & 3.57 & 3.45 & 3.26 & 3.59 & 3.78 & 3.47 & 3.10 & 3.21 \\
\hline Delaware ............................. & 2.65 & 2.92 & 2.78 & 2,30 & 2.42 & 2.44 & 2.97 & 2.41 \\
\hline District of Columbia ........ & - & -. & - & $-\cdots$ & -- & -- & - & $\cdots$ \\
\hline 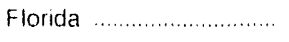 & 2.58 & 2.61 & 2.46 & 2.16 & 2.31 & 2.37 & 2.45 & 2.48 \\
\hline 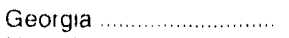 & 3.45 & 3.63 & 3.74 & 3.05 & 3.16 & 3.24 & 2.74 & 3.19 \\
\hline Hawaii ............................... & 6.80 & 6.49 & 6.36 & 6.28 & 5.85 & 6.24 & 7.29 & 6.96 \\
\hline 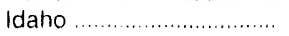 & 2.12 & 2.17 & 2.29 & 2.24 & 2.20 & 2.77 & 2.15 & 2.09 \\
\hline 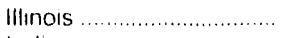 & 3.06 & 3.02 & 2.68 & 2.64 & 2.57 & 2.75 & 2.48 & 2.85 \\
\hline Indiana .............................. & 3.13 & 3.21 & 3.10 & 2.78 & 3.38 & 3.50 & 3.12 & 2.80 \\
\hline 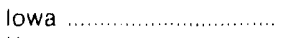 & 2.92 & 2.86 & 2.98 & 2.85 & 2.98 & 3.06 & 2.79 & 2.48 \\
\hline Kansas $\ldots \ldots \ldots \ldots \ldots \ldots \ldots$ & 2.78 & 2.07 & 2.02 & 2.34 & 2.27 & 2.25 & 2.42 & 2.87 \\
\hline 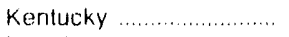 & 3.21 & 3.07 & 297 & 2.49 & 2.73 & 3.05 & 3.28 & 3.29 \\
\hline Loussiana .......................... & 2.93 & 2.97 & 3.03 & 2.58 & 2.73 & 2.64 & 2.84 & 2.82 \\
\hline 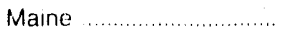 & 2.99 & 3.28 & 2.94 & 2.67 & 2.72 & 3.05 & 2.79 & 2.69 \\
\hline 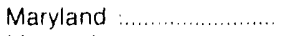 & 3.05 & 3.27 & 3.09 & 3.85 & 3.99 & 3.99 & 3.45 & 2.80 \\
\hline Massachusetts .............. & 3.21 & 3.12 & 2.91 & 2.84 & 2.81 & 3.28 & 2.84 & 2.96 \\
\hline 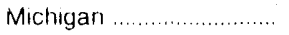 & 3.13 & 3.31 & 3.39 & 2.95 & 3.14 & 3.04 & 2.52 & 3.11 \\
\hline Minnesola .................... & 2.86 & 2.77 & 2.85 & 2.87 & 3.09 & 3.22 & 2.66 & 2.35 \\
\hline Mississippi ..................... & 2.90 & 3,12 & 3.34 & 2.65 & 2.65 & 2.77 & 2.64 & 2.67 \\
\hline Missouri ......................... & 3.18 & 2.92 & 2.86 & 3.00 & $3.5 \%$ & ค 3.55 & 3.40 & 2.71 \\
\hline 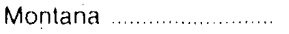 & 3.60 & 3.57 & 3.80 & 5.21 & ค 3.37 & R 4.26 & R 3.71 & R 4.03 \\
\hline Nebraska ........................... & 2.98 & 2.97 & 3.07 & 2.93 & 3.10 & 3.20 & 2.85 & 2.59 \\
\hline 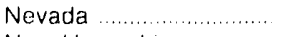 & 2.95 & 3.46 & 2.81 & 2.67 & 3.00 & 2.68 & 2.38 & 2.64 \\
\hline New Hampshire ............. & 3.51 & 3.27 & 3.06 & 2.95 & 3.32 & 3.12 & 2.91 & 3.06 \\
\hline New Jersey . ...................... & 3.17 & 3.14 & 2.97 & 3.27 & 3.14 & 3.11 & 2.81 & 2.88 \\
\hline New Mexico ....................... & 2.67 & 2.61 & 2.55 & 2.00 & 3.06 & 3,41 & 3.40 & 2.40 \\
\hline 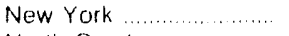 & 3.00 & 3.01 & 2.79 & 2.74 & 2.77 & 2.76 & 2.63 & 2.92 \\
\hline North Carolina ................ & 2.85 & 3.10 & 2.82 & 2.74 & 2.86 & 2.93 & 2.92 & 2.64 \\
\hline North Dakota …............. & 3.40 & 3.26 & 3.52 & 4.23 & 3.17 & 4.01 & 3.75 & 3.54 \\
\hline 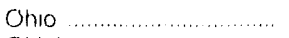 & 3.07 & 3.28 & 3.16 & 2.88 & 3.03 & 3.12 & 3.03 & 2.74 \\
\hline 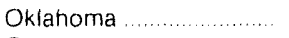 & 2.01 & 2.05 & 2.24 & 1.74 & 1.73 & 1.86 & 1.83 & 2.01 \\
\hline Oregon & 2.51 & 2.65 & 2.96 & 2.53 & 2.61 & 2.90 & 2.51 & 2.55 \\
\hline Pennsylvania ................... & 3.45 & 3.31 & 3.09 & 4.17 & 4.17 & 3.74 & 3.51 & 3.39 \\
\hline Rhode Island .................... & 3.70 & 3.72 & 3.39 & 3.84 & 4.21 & 4.07 & 3.79 & 3.32 \\
\hline South Carolına ................ & 3.14 & 3.53 & 3.39 & 2.54 & 2.65 & 3.15 & 3.24 & 3.12 \\
\hline South Dakota ............... & 3.24 & 3.16 & 3.23 & 4.04 & 3.47 & 3.89 & 3.31 & 2.84 \\
\hline 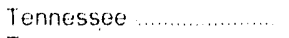 & 2.83 & 2.76 & 2.70 & 2.60 & 2.89 & 2.79 & 2.65 & 2.54 \\
\hline 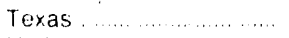 & 3.11 & 3.31 & 2.98 & 3.14 & 2.88 & 2.86 & 2.93 & 3.01 \\
\hline 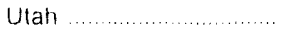 & 3.89 & 3.55 & 3.07 & 4.87 & 5.27 & 5.23 & 4.75 & 4.55 \\
\hline 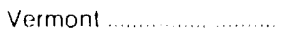 & 2.86 & 2.61 & 2.62 & 2.76 & 3.49 & 3.42 & 2.84 & 2.70 \\
\hline 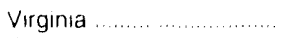 & 3.04 & 3.14 & 2.78 & 2.60 & 3.35 & 3.68 & 2.97 & 2.61 \\
\hline Washington $\ldots . . . . . . . . . .$. & 1.96 & 2.26 & 2.36 & 1.84 & 1.87 & 1.83 & 1.71 & 1.82 \\
\hline 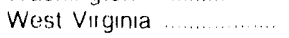 & 3.66 & 3.83 & 3.30 & 6.19 & 5.29 & 4.89 & 3.95 & 3.52 \\
\hline 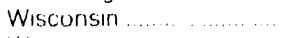 & 336 & 377 & 3.63 & 3.99 & 3.90 & 3.87 & 3.22 & 3.05 \\
\hline Wyoming ...................... & 3.20 & 3.02 & 3.20 & 4.20 & 3.28 & 3.65 & 4.76 & 2.97 \\
\hline Total & 3.01 & 3.01 & 2.88 & 2.91 & 3.03 & 3.00 & 2.81 & 2.84 \\
\hline
\end{tabular}

See footnotes at end of table 
Table 27. Average City Gate Price by State (Continued) (Dollars per Thousand Cubic Feet)

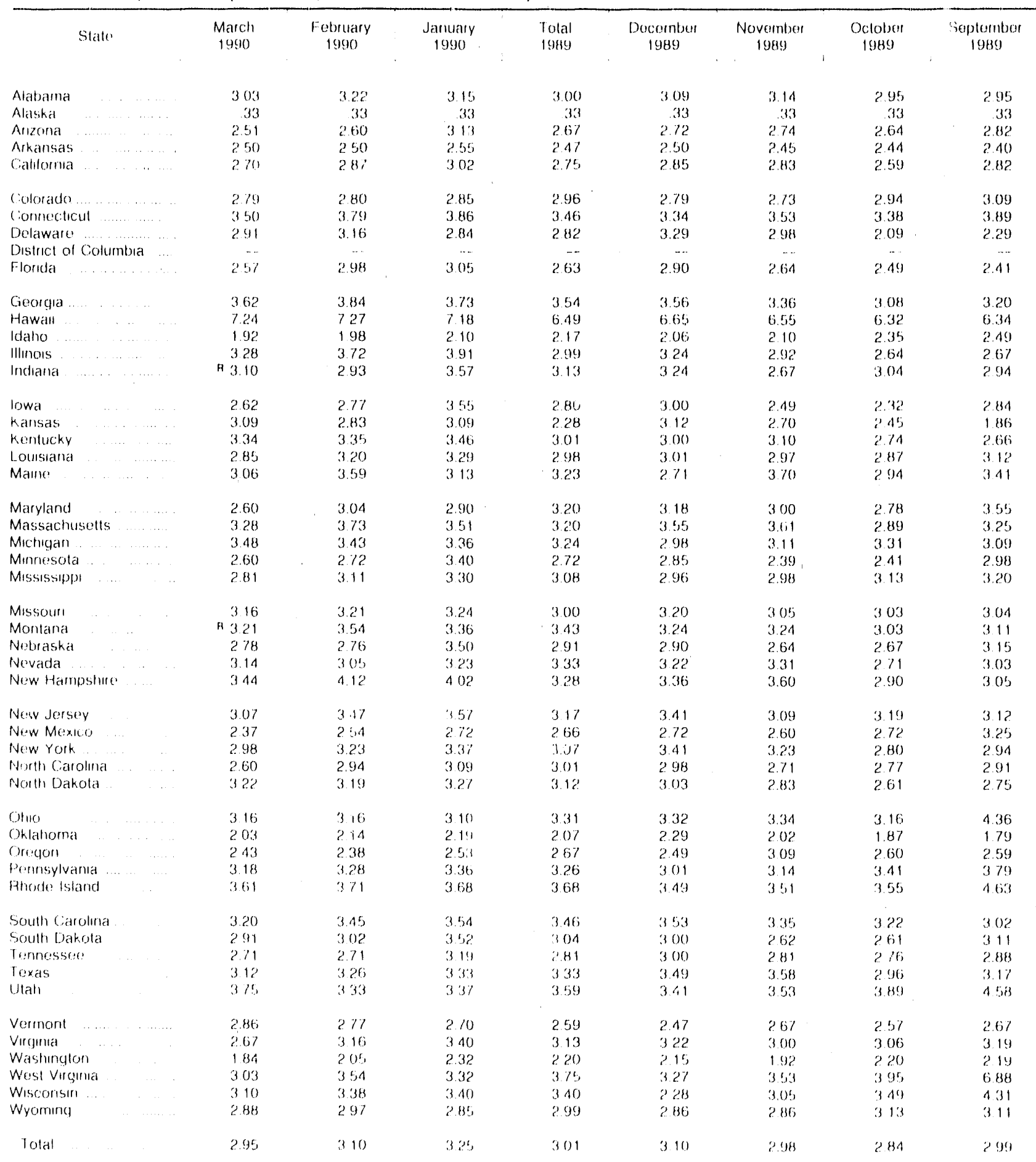

Soe footroles it end of lable: 
Table 27. Average City Gate Price by State (Continued) (Dollars per Thousand Cublc Feet)

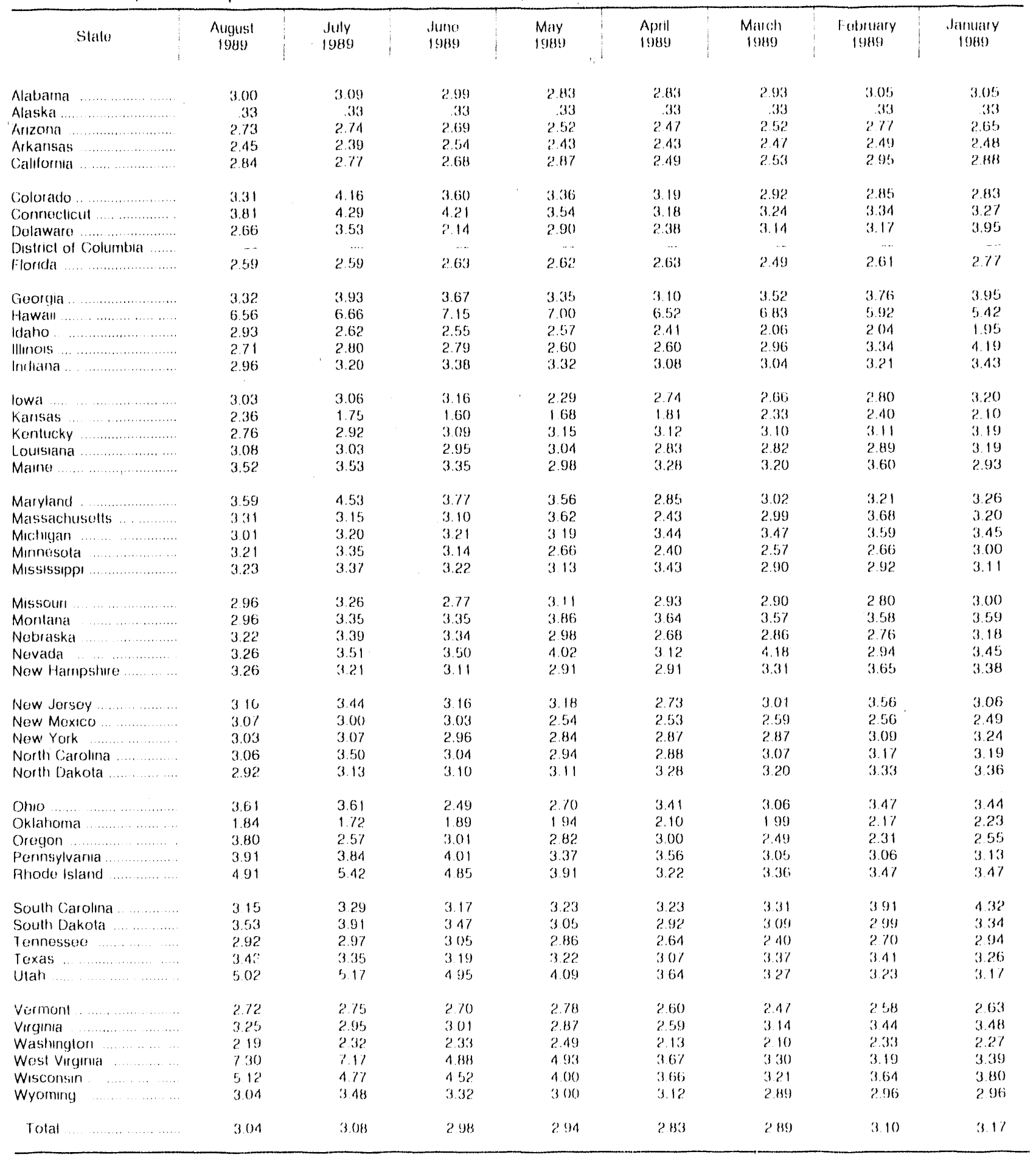

h Hervised Data

Not Applicatsole

Noles and Sources Soe thu last pago of thes section. 
Table 28. Average Price of Natural Gas Delivered to Residential Consumers by State

\section{(Dollars per Thousand Cubic Feet)}

\begin{tabular}{|c|c|c|c|c|c|c|c|c|}
\hline Slate & $\begin{array}{l}\text { YTD } \\
1960\end{array}$ & $\begin{array}{l}Y 70 \\
1980\end{array}$ & $\begin{array}{l}Y 1 D \\
10483\end{array}$ & $\begin{array}{c}\text { Augusi } \\
1000\end{array}$ & $\begin{array}{l}\text { July } \\
1090\end{array}$ & $\begin{array}{l}\text { Jumos } \\
\text { lgyo0 }\end{array}$ & $\begin{array}{l}\text { May } \\
1900\end{array}$ & $\begin{array}{l}\text { April } \\
1690\end{array}$ \\
\hline Alabarna & $6: 30$ & 612 & 6.45 & 860 & 8.49 & 0.15 & 7.11 & 6.38 \\
\hline Alaskil & 3.81 & 363 & 3.30 & 4.21 & 4.30 & 4.10 & 3.93 & 3.79 \\
\hline Arrzona & 6.76 & $6 ! n$ & 6.866 & 937 & 9.10 & 8,37 & 7.77 & 7.02 \\
\hline Arkankials & 4.93 & 4.77 & 4.73 & 6.83 & 6.694 & 6.24 & 3.39 & 1.834 \\
\hline Califomal $\ldots \ldots$ & 5.74 & 5.60 & 5.54 & 5.90 & 6.07 & 6.22 & 5.95 & 5.07 \\
\hline Colorado & 4.52 & 4.56 & 4.37 & 5.95 & 5,86 & $5.0 \mathrm{~d}$ & 4.55 & 1.37 \\
\hline comochoult ..... . ... & 8.56 & 8.20 & 7.80 & 10.46 & 10.09 & 9.91 & 9.25 & 8.39 \\
\hline Dolaware $\quad$....... ... & 6.39 & 6.36 & 5.86 & 8.35 & 8.09 & 7.74 & 6.834 & 6.35 \\
\hline District of columbial .... & 750 & 7.37 & 6.73 & 6.97 & 6.963 & 6.81 & 7.37 & 7.21 \\
\hline Flonda $\ldots$ & 8.17 & 8.00 & 7.56 & 9. 92 & 9.65 & 9.36 & 89.97 & B3.36 \\
\hline Georgla & 6.90 & 6.29 & 6.31 & 8.23 & 8.is & 7.78 & 7.10 & 6.383 \\
\hline Hawall . . . . . . . . & 15.783 & 15.51 & 15.78 & 15,11 & 14.96 & 15.68 & 16.32 & 10.28 \\
\hline 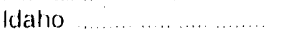 & 496 & 5.00 & 5.61 & 6.61 & 6.32 & 5.47 & 5.34 & 5.17 \\
\hline Illinols $\ldots \ldots \ldots \ldots$ & 5.11 & 5.04 & 4.59 & 6.14 & 6.15 & $\$ .79$ & 5.17 & d.88 \\
\hline Indiana ................ & 5,74 & 580 & 5.17 & 6.54 & 6.77 & 6.60 & 5.98 & 5.56 \\
\hline lowa ... . . . . & 499 & 4.64 & 4.79 & 6.88 & 6.66 & 5.61 & 1.80 & 4.51 \\
\hline Kansals . & 4.16 & 4.05 & 3.98 & 5.70 & 5.64 & 5.17 & 4.63 & 4.40 \\
\hline Konlucky _.. & 4.93 & 4.70 & 4.43 & 626 & 6.46 & 5.84 & 5.50 & 1.78 \\
\hline Loumsianda & 5.84 & 570 & 5.19 & $7.7 \%$ & 1.10 & 7.32 & 116.20 & 6.11 \\
\hline Mamo , ............ & 7.78 & 7.01 & 731 & 8.37 & 8.31 & 7.56 & 7.77 & 8.27 \\
\hline 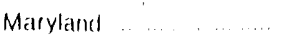 & 6.35 & 6.28 & 5.78 & 8.16 & 793 & 7.40 & 6.78 & 6.11 \\
\hline Massachusetls & 772 & 7.10 & 6.31 & $\$ 29$ & 7.87 & 6.95 & 6.73 & 3.39 \\
\hline Michugan & 4.97 & 5.26 & 5.23 & 6.56 & $6.6 !$ & 5.88 & 526 & 1.86 \\
\hline Mirmosolá ... ....... & 4.56 & 4.57 & 4.60 & 463 & 5.07 & 4.77 & 4.40 & 1.13 \\
\hline Misisisistppl & 5.16 & 5.14 & 5.90 & $5.5 ?$ & 5.43 & 5.37 & 5.57 & 5.01 \\
\hline$\ldots \ldots \ldots$ & 6.13 & 477 & $4 i ?$ & 7.06 & fi.65 & 6.11 & 5.30 & 5,01 \\
\hline Moritana ......... & 4.48 & 4.39 & 4.37 & 591 & ค 576 & 4.94 & $4.6 ?$ & 14.45 \\
\hline Nebratsa & $45 \%$ & 4.45 & 442 & 514 & 5.26 & 5.04 & 454 & 4.23 \\
\hline Nevada & 560 & 552 & $5 / 4$ & 7.35 & 7.08 & 6.48 & 6.36 & 6.03 \\
\hline New Hampslines & 7.42 & 678 & $6.0 \%$ & 8.51 & 8.32 & 7.61 & 7083 & 7.77 \\
\hline New Jertiey & 6.60 & 645 & 6.26 & 892 & 8.69 & H.OB & $7.3 t$ & 6.69 \\
\hline New Mexnos & 5.56 & 5.63 & 4.96 & 7.81 & 758 & 6.96 & 602 & 5.51 \\
\hline Now rork.... & 7.31 & 7.16 & 6.28 & 9.80 & 9.96 & 8.20 & 7.583 & 708 \\
\hline North Carcuiria & 6.06 & 6.58 & 6.16 & 8.10 & 6.13 & 7.90 & 6.79 & 5.833 \\
\hline North Dakola ....... & 465 & 4.67 & 5.21 & $68 \%$ & 6.18 & 545 & 4.85 & 1.54 \\
\hline Ohno & 5,25 & 5.44 & 5.14 & 652 & 6.48 & 5.93 & 321 & 528 \\
\hline$\ldots \ldots$ & 468 & 4.34 & 450 & 673 & 6.66 & 6.20 & 5.21 & $4.6 ?$ \\
\hline Orogon & 6.23 & 613 & 6.68 & 739 & 701 & 656 & 6.40 & 6.24 \\
\hline Punnsylvanla $\quad \ldots . . . .$. & 651 & $60 \%$ & 5.64 & 864 & 848 & 758 & 698 & 0.14 \\
\hline Rhode Island & 7.11 & 711 & 6.49 & 835 & $83: 34$ & 117.74 & 7.34 & 7.07 \\
\hline South Carcolina & 7.06 & 6.69 & 6.73 & \&S BSB & 874 & 33.29 & 7.32 & 6.963 \\
\hline Soulh Dakota & 502 & 484 & 487 & 647 & 681 & 5.93 & 5.12 & 472 \\
\hline Tennesser: & 5.03 & 4.76 & $4,5,5$ & 656 & $6 ?$ & 500 & 5.34 & 4.98 \\
\hline 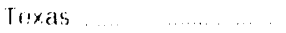 & $57 \%$ & 5,51 & 5,25 & $7: 30$ & 7.13 & 6.836 & $6.4 !$ & 5.983 \\
\hline Utah $\ldots \ldots \ldots \ldots \ldots$ & 5.22 & 504 & 5.06 & 503 & 5.81 & 5.30 & 5.28 & 5.44 \\
\hline Verrnoril & $56 ?$ & 5.60 & 5,63 & 7.11 & 7.100 & (6.20) & 584 & b, \\
\hline Virginata & 656 & 659 & 5.57 & 83.78 & $\$ 370$ & 719 & 714 & (j) 19 \\
\hline Wasturigton & $51: 3$ & 5.48 & 5,46 & 7.06 & 649 & 561 & 5.283 & 500 \\
\hline West Vifgina & $6: 30$ & 5,94 & 5,50 & 372 & 83.30 & 737 & 683 & 6.31 \\
\hline Wiscontin & 574 & 568 & $59 ?$ & 639 & 669 & 600 & s & 5.54 \\
\hline Wyorning & 479 & 4.666 & 442 & $5 \%$ & 58 & 479 & 4.89 & 476 \\
\hline Tolal & 5,75 & $565_{1}$ & 5.39 & 7.04 & 699 & 696 & 5.97 & 5.62 \\
\hline
\end{tabular}

See loomoles al end of latse 
Table 28. Average Price of Natural Gas Delivered to Residential Consumers by State (Continued) (Dollars per Thousand Cubic Feet)

\begin{tabular}{|c|c|c|c|c|c|c|c|c|}
\hline Stato & $\begin{array}{l}\text { March } \\
1090\end{array}$ & $\begin{array}{c}\text { Fobruary } \\
1090\end{array}$ & $\begin{array}{c}\text { Jantualy } \\
19000\end{array}$ & $\begin{array}{l}\text { Tolal } \\
\text { la869 }\end{array}$ & $\begin{array}{l}\text { Decominor } \\
\text { l989 }\end{array}$ & $\begin{array}{l}\text { Noverntion } \\
\text { lats }\end{array}$ & $\begin{array}{l}\text { Oclober } \\
\text { lisg9 }\end{array}$ & $\begin{array}{c}\text { Senplember } \\
1989\end{array}$ \\
\hline 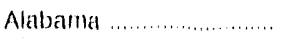 & 6.12 & 5.92 & $5.5,3$ & $6.8 \%$ & $5(3)$ & $6.7 \%$ & 7.87 & 8.52 \\
\hline 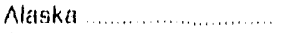 & 3.71 & 3.68 & 370 & 3.43 & 3.95 & 3.57 & 3.71 & 3.94 \\
\hline Arizona $\ldots . . . . . . . . . . . . . . . . .$. & 6.38 & 6.20 & 6.17 & $6.6 \mathrm{~A}$ & 0.08 & 7.26 & $\mathrm{H}, \mathrm{BH}$ & 9.30 \\
\hline 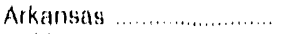 & 4.81 & 4.66 & 4.41 & 4.86 & 4.41 & 5.09 & 0.15 & 6.67 \\
\hline 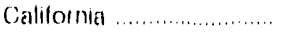 & 5.619 & 5.77 & 5.72 & 5.50 & 538 & $6.1 ?$ & (i.11 & 0.10 \\
\hline 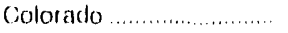 & 4.27 & 4.46 & 9.30 & 4.63 & 4.49 & 4.75 & 3.27 & 5.81 \\
\hline Conner licut .................... & 8.22 & 8.24 & 8.17 & 8.25 & 7.60 & $8.2 \%$ & 0.06 & 10.00 \\
\hline 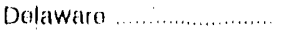 & 6.18 & 6.15 & 5.95 & 6.12 & 6.16 & 6.76 & 7.383 & 7.40 \\
\hline District of Columbia ...... & 8.06 & $7 . B A$ & 7.37 & 7.44 & 7.31 & 7.61 & 8.01 & 8.60 \\
\hline Floridat ........................... & 8.18 & 8.01 & 6.95 & 8.06 & 7.18 & 8.34 & 0.52 & 9.30 \\
\hline 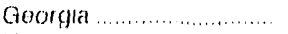 & 6.76 & 6.89 & 6.45 & 6.24 & 562 & 6.40 & 7.28 & 7.47 \\
\hline 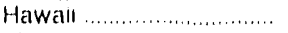 & 16.12 & 15.79 & 15.81 & 15.66 & 15.89 & 15.90 & 15.99 & 15.98 \\
\hline 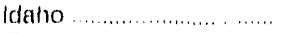 & 4.83 & 4.68 & 4.64 & 5.05 & 4.81 & 5.30 & 5.67 & 5.95 \\
\hline Illinols . ......................... & 1.98 & 5.2 .5 & 1.75 & $4.9 ?$ & 4.54 & 4.56 & 5.00 & 5.81 \\
\hline Indiana .......................... & 5.50 & 5.54 & 5.75 & 5.51 & $4.8 A$ & 491 & 6.14 & 6.16 \\
\hline lowa & 4.6\% & 4.95 & 5.05 & 4.70 & 4.60 & 1.74 & 0.12 & 6.17 \\
\hline 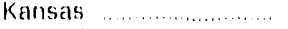 & 4.34 & 4.32 & 4.21 & 4.17 & 4.24 & 4.12 & 4.53 & 6.35 \\
\hline 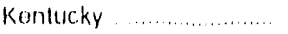 & 4.61 & 4.75 & 468 & 4.68 & 4.30 & 4.66 & 6.12 & 5.98 \\
\hline Loulsiallá ........................ & 5.01 & 5.90 & 5.18 & 5.97 & 6.01 & 6.83 & 7.36 & 7.63 \\
\hline Maino ......................... & $H .0 B$ & 7.96 & 7.00 & 7.16 & 708 & 7.50 & 7.19 & 8.69 \\
\hline 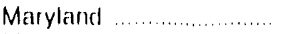 & 6.17 & 6.29 & 5.81 & 6.30 & 5.71 & 6.46 & 7.29 & 8.11 \\
\hline Massachusolts ............... & 8.12? & 8.02 & 7.25 & 7.16 & 7.31 & 7.77 & 6.31 & 7.99 \\
\hline Mıchlyan ......................... & 4.76 & 4.72 & 1.66 & 5.19 & 468 & 4.94 & 5.51 & 6.60 \\
\hline 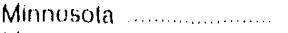 & 4.20 & 4.57 & 4.98 & $4.5 \%$ & 4.56 & 4.45 & 4.60 & 5.19 \\
\hline 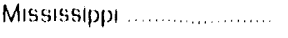 & 5.30 & 5.25 & 4.97 & 5.10 & 4.73 & 5.28 & 5.35 & 5.47 \\
\hline 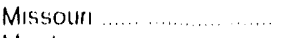 & 5.02 & 4.99 & 4.73 & 4.84 & 4.65 & $5.0 ; 3$ & 5.40 & 6.35 \\
\hline Montana ........................ & A 4.33 & 4.24 & 4.23 & 4.37 & 4.26 & 4.20 & 4.35 & 4,58 \\
\hline 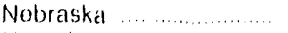 & 4.22 & 4.44 & 4.66 & 4.481 & 4.37 & 4.47 & 4.80 & 5.22 \\
\hline Nuvada ........................... & 5.46 & 5.05 & 5.07 & 5.55 & 5.18 & 5.53 & 6.39 & 6.60 \\
\hline Now Hampshiro ............. & 7.63 & 7.60 & 6.76 & 6.85 & 6.83 & 7.28 & 6.86 & 7.43 \\
\hline Now Jorsey ..................... & 6.46 & 6.43 & 5.63 & 6.51 & 6.10 & 6.62 & 7.283 & 8.28 \\
\hline Now Moxico ................. & 5.23 & 5.20 & 5.10 & 5.73 & 5.28 & 5.883 & 7.49 & 7.93 \\
\hline Now York ........................ & 7.05 & ?.26 & 6.72 & 7.22 & 6.77 & 7.58 & 7.98 & 9.18 \\
\hline North Carolina ............ & 5.81 & 5.77 & 5.69 & 6.55 & 5.99 & 6.61 & 7.44 & 8.68 \\
\hline North Dakota .................. & 4.50 & 4.30 & 4.38 & 4.66 & 4.34 & 4.65 & 5.22 & 5.08 \\
\hline 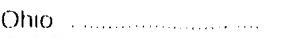 & 4.97 & 5.06 & 5.15 & 5.32 & 4.90 & 5.21 & 5.29 & 6.283 \\
\hline Oklahoma ...................... & 4.47 & 4.383 & 4.18 & 4.50 & 4.35 & 5.00 & 5.97 & 6.34 \\
\hline 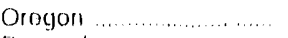 & 6.06 & 6.07 & 6.06 & 6.19 & 6.16 & $6.2 \%$ & 6.76 & 7.13 \\
\hline Ponnsylvanla ................ & 6.27 & 6.22 & 6.07 & 6.14 & 6.01 & 6.483 & 6.92 & 7.84 \\
\hline Phode lsland .................... & 6.88 & 6.93 & 6.83 & 7.13 & 6.84 & 7.10 & 7.19 & 8.55 \\
\hline South Carolma .................. & 6.92 & 6.86 & 6.866 & 6.73 & 6.698 & 6.79 & 7.02 & 7.88 \\
\hline Soulh Dakota ............. & 4.67 & $4.8 ?$ & 4.98 & 1.85 & 4.83 & 467 & 5.00 & 5.73 \\
\hline Tonnessoe ................ & 4.98 & 4.99 & 4.10 & 1.833 & $4.7 \%$ & 5.09 & 6.54 & 6.03 \\
\hline 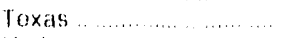 & $5.6 ?$ & 5.36 & 1.56 & 6.55 & 503 & 5.65 & 6.46 & 6.73 \\
\hline 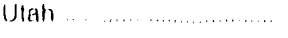 & 515 & 5.05 & 506 & 5.14 & 5.17 & 5.39 & 5.85 & 5.80 \\
\hline 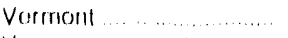 & 5.47 & 5.45 & 3.40 & $\$ 62$ & 5.43 & 5.69 & 599 & 6.8B \\
\hline 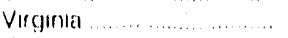 & 6.02 & 653 & 6.22 & 6.59 & 6.19 & 6.48 & 7.09 & 0.13 \\
\hline Washongton ................. & 401 & 4.86 & 4.92 & 5.10 & 5.09 & 5.45 & 6.25 & 7.08 \\
\hline Wost Virginlal ................ & 6.25 & 6.19 & 590 & 5.73 & 6.17 & 5.46 & 5.91 & 6.86 \\
\hline Wiscorsin ........................ & 579 & 581 & 5.54 & 5.64 & 5.38 & 563 & 5,58 & 6.?.1 \\
\hline Wyommng $\ldots \ldots . . . . . . . . .$. & 471 & 4.70 & 464 & 4.71 & 4.61 & 4.77 & 5.11 & 5.49 \\
\hline Tolal & 5.54 & 5.63 & 5.42 & 5.64 & 6.30 & 6.66 & 6.06 & 6.80 \\
\hline
\end{tabular}

Soe lootnotes at end of table 


\section{Table 28. Average Price of Natural Gas Delivered to Residential Consumers by State (Continued)}

(Dollars per Thousand Cubic Feet)

\begin{tabular}{|c|c|c|c|c|c|c|c|c|}
\hline Silato & $\begin{array}{c}\text { Augusl } \\
\text { I9)(39) }\end{array}$ & $\begin{array}{l}\text { July } \\
\text { logs }\end{array}$ & $\begin{array}{l}\text { Juno } \\
1989\end{array}$ & $\begin{array}{l}\text { May } \\
1989\end{array}$ & $\begin{array}{l}\text { April } \\
\text { Ig) }\end{array}$ & $\begin{array}{c}\text { March } \\
19889\end{array}$ & $\begin{array}{c}\text { Fobruary } \\
1989\end{array}$ & $\begin{array}{c}\text { January } \\
\text { Igng }\end{array}$ \\
\hline 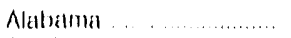 & 8.47 & 8.32 & 7.83 & 0.73 & 6.01 & 5.55 & 5.71 & 5,65 \\
\hline 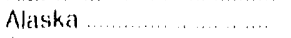 & 4.19 & 4.07 & 3.84 & 3.72 & 3.61 & 3.56 & 3.51 & 3.63 \\
\hline Arizona ................. & 9.55 & 0.04 & 8.36 & 8.12 & 7.46 & 6.65 & 0.25 & 6.23 \\
\hline Arkinnsas & 6.79 & 6.58 & 6.11 & 5.75 & 1.97 & 1.35 & 4.31 & 4.33 \\
\hline Calliornia ................ & 5.90 & 6.07 & 6.09 & 5.69 & 1.62 & 5.21 & 5.63 & 5.84 \\
\hline 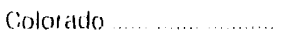 & 6.07 & 5.70 & 5.20 & $4.8 \%$ & 4.54 & 4.33 & 4.30 & 4.30 \\
\hline Connucticul $\quad \ldots . . . . .$. & 10.18 & 10.01 & 9.94 & 8.76 & 8.27 & 7.78 & 7.80 & 7.69 \\
\hline Dolawatre! & 7.49 & 7.96 & 7.52 & 6.81 & 6.33 & 6.06 & 6.10 & 6.06 \\
\hline Disilred of Columbia .... & 6.70 & 7.11 & 5.91 & 7.13 & 7.50 & 7.72 & 7.413 & 7.49 \\
\hline 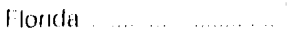 & 9.67 & 9.41 & 9.31 & 8.91 & 7.92 & 7.12 & 7.55 & 7.26 \\
\hline 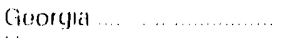 & $7.9 \%$ & 7.91 & 7.75 & 7.03 & 6.2 .3 & 6.14 & 5.81 & 5.77 \\
\hline 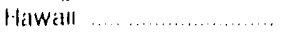 & 16.47 & 16.37 & 16.23 & 15.76 & 15.49 & 15.12 & 14.31 & 14.67 \\
\hline 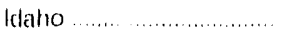 & 650 & 6.38 & 5.66 & 5.56 & 5.30 & 4.92 & 1.71 & 4.69 \\
\hline Illinots ........................... & 6.43 & 5.85 & 5.60 & 5.11 & 4.99 & 5.08 & 4.96 & 4.70 \\
\hline 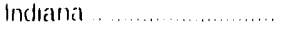 & 6.87 & 6.88 & $6.7 i$ & 6.19 & 5.86 & 5.62 & 5.48 & 5.72 \\
\hline lowa $\ldots \ldots \ldots \ldots \ldots$ & 0.6 .1 & 6.383 & 5.78 & 4.77 & 4.32 & 4.25 & 4.19 & 4.67 \\
\hline 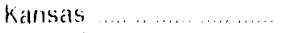 & 5.67 & 5.52 & 5.26 & 4.84 & 4.05 & 3,80 & 3.77 & 3.73 \\
\hline 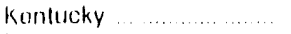 & 6.12 & 5.98 & 5.50 & 5.03 & 4.71 & 4.46 & 4.49 & 4.48 \\
\hline Loulsianá $\ldots . \ldots . . . . .$. & 7.65 & 7.23 & 7.11 & 6.43 & 5.78 & 4.90 & 5.23 & 5.40 \\
\hline 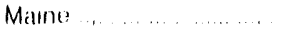 & 8.47 & 8.06 & 7.06 & 7.21 & 7.01 & $6.7 \%$ & 6.77 & $6 . \%$ \\
\hline 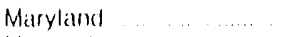 & 789 & 7.70 & 7.26 & 6.57 & 0.20 & 6.01 & 6.06 & 5.92 \\
\hline Massirachuselts ............ & 8.20 & 7.88 & 7.44 & 6.60 & 7.12 & 7.02 & 7.06 & 6.99 \\
\hline Michugan $\ldots . . . . . . . . . . .$. & 713 & 6.86 & 6.37 & 5.48 & 5.13 & 5.02 & 5.02 & 5.03 \\
\hline Munnosola ............. & 5.40 & 5.34 & 5.08 & 4.64 & 4.32 & 4.31 & 4.50 & 4.68 \\
\hline Mississipp $\ldots \ldots \ldots \ldots$ & 5.51 & 5.49 & 5.113 & 5.57 & 5.11 & 4.93 & 5.10 & 5.0 ? \\
\hline Missoum ................. & 6.73 & 6.56 & 6.23 & 5.35 & 4.75 & 4.48 & 4.47 & 4.45 \\
\hline 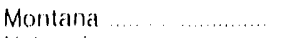 & 4.67 & 4.72 & 4.57 & 4.52 & 4.41 & 4.35 & 4.33 & 4.29 \\
\hline 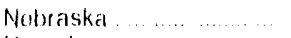 & 5.46 & 5.34 & 5.65 & 4.70 & 4.30 & 4.15 & 4.31 & 4.45 \\
\hline 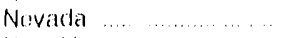 & $7.2 \%$ & 6.90 & 6.28 & 6.12 & $5.8 ?$ & 5.34 & 5.09 & 5.10 \\
\hline Now Hampshour ......... & 794 & 7.50 & 7.00 & 6.32 & 6.91 & 6.75 & 6.75 & 6.66 \\
\hline Now Jorsoy . .............. & 8.54 & 8.27 & 7.86 & 6.96 & 6.41 & 6.15 & 6.14 & 5.95 \\
\hline Now Moxico ................ & 7.09 & 7.82 & $? 42$ & 7.08 & 5.86 & 5.21 & 5.01 & 5.10 \\
\hline Now York .................... & 9.36 & 9.01 & 8.31 & 7.11 & 6.837 & 7.46 & 6.66 & 6.60 \\
\hline North Caroluna .............. & 8.97 & 8.71 & 8.02 & 6.99 & 6.80 & 6.29 & 6.30 & 6.17 \\
\hline North Dakola ............. & 6.64 & 6.08 & 5.51 & 5.09 & 4.50 & 4.41 & 4.46 & 4.47 \\
\hline Ono $\ldots \ldots \ldots \ldots \ldots \ldots$ & 6.54 & 6.56 & 6.26 & 5.31 & $5.4 \%$ & 6.25 & 5.33 & 5.33 \\
\hline 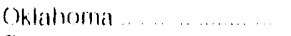 & 6.55 & 6.35 & 6.06 & 5.45 & 4.60 & 3.98 & 3.85 & 3.88 \\
\hline Orogon $\quad \ldots . \quad \ldots n \ldots$ & 7.21 & 6.80 & 6.56 & 6.56 & 6.03 & 5.85 & 5.85 & 6.30 \\
\hline Ponmsylvanlat ................. & 4.06 & 791 & 6.77 & 6.26 & 5.94 & 5.78 & 5.75 & 5.73 \\
\hline Fhodo lsand .... ....... & 865 & 8.44 & 8.25 & 7.26 & 7.13 & 6.98 & 7.00 & 6.56 \\
\hline South Carolma ........ & 8.19 & 7.75 & 7.44 & 6.64 & 6.54 & 6.66 & 6.63 & 6.53 \\
\hline Soult Dakolal .............. & 626 & 6.01 & 5.53 & 503 & 4.65 & 4.54 & 4.67 & 4.86 \\
\hline Tonnosisoo .................. & 611 & 5.99 & 5.50 & 5.08 & 4.81 & 4.53 & 4.60 & 1.57 \\
\hline 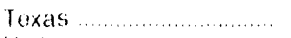 & 7.18 & 6.97 & 6.80 & 6.53 & 5.833 & 5.2 .1 & 5.10 & 5.11 \\
\hline 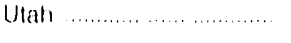 & 5.80 & 5.51 & 6.17 & 5.27 & 5.26 & 5.01 & 4.88 & 4.85 \\
\hline Vormont .............. & 7.08 & 6.96 & 6.43 & 5.83 & 5.54 & 5.44 & 5.44 & 5.41 \\
\hline 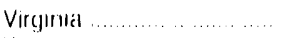 & 878 & B.67 & 8.26 & 6.52 & 6.51 & 6.35 & 6.38 & 6.17 \\
\hline Washmrigton & 752 & $70 \%$ & 6.40 & 6.12 & 5,17 & 5.19 & 5.15 & 5.183 \\
\hline West Viryunla $\ldots . . . . . . .$. & 724 & 7.07 & 6.64 & 5.80 & 5.48 & 5.35 & 5.31 & 5.29 \\
\hline Wiscorisin & 676 & 6.43 & 6.04 & 5.59 & 5.57 & 5.55 & 5.70 & 5.61 \\
\hline Wyoming $\ldots$ & 5.184 & 6,53 & 504 & $4.8 ?$ & 1.62 & $4.6 \%$ & 4.49 & 4.49 \\
\hline$\ldots \ldots \ldots \ldots$ & 7.07 & 6.92 & 6.68 & 5.93 & 5.54 & 5.45 & 5.38 & 5.41 \\
\hline
\end{tabular}

n Hevised loata

Noles and Souncos: Soe tho last page of thes soction. 
Table 29. Average Price of Natural Gas Sold to Commercial Consumers by State

(Dollars per Thousand Cublc Feet)

\begin{tabular}{|c|c|c|c|c|c|c|c|c|}
\hline Slale & $\begin{array}{l}\text { YTD } \\
1990\end{array}$ & $\begin{array}{l}Y T D \\
1989\end{array}$ & $\begin{array}{l}\text { YTO } \\
1988\end{array}$ & $\begin{array}{c}\text { August } \\
1990\end{array}$ & $\begin{array}{l}\text { July } \\
1990\end{array}$ & $\begin{array}{l}\text { Juno } \\
1990\end{array}$ & $\begin{array}{l}\text { May } \\
1990\end{array}$ & $\begin{array}{l}\text { Apr } 1 \\
1991)\end{array}$ \\
\hline Alaberma ......................... & 5.26 & 5.11 & 5.33 & 5.38 & 5.50 & 5.91 & 6.45 & 5.20 \\
\hline 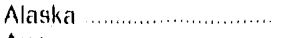 & 2.62 & 2.64 & 2.55 & 2.41 & 2.41 & 2.52 & 2.55 & 2.62 \\
\hline Arizona & 4.78 & 1.79 & 4.95 & 4.76 & 4.73 & 4.66 & 4.67 & 4.79 \\
\hline Arkanisass ........................ & 1.39 & 1.35 & 4.33 & 4.75 & 4.67 & 4.68 & 146 & 4.34 \\
\hline Galiforna .......................... & 5.15 & $4.8 \%$ & 4.86 & 4.66 & 4.40 & 4.37 & 4.54 & 5.29 \\
\hline 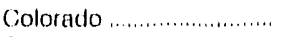 & 3.95 & 4.02 & 3.87 & 4.28 & 4.24 & 4.00 & 3.95 & 3.89 \\
\hline Connoclicul ..................... & 6.34 & 5.97 & 5.67 & 5.02 & 5.05 & 115 & 5.88 & 6.09 \\
\hline 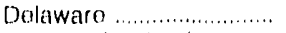 & 5.43 & 5.38 & 4.82 & 5.41 & $5.6 \%$ & 5.60 & 5.54 & 5.43 \\
\hline District of Columbia ...... & 5.70 & 5.28 & 5,03 & 4.80 & 5.12 & 5.2 .1 & 5.09 & 5.41 \\
\hline Florida & 4.85 & 4.85 & 4.53 & 4.60 & 4.64 & 4.66 & 4.68 & 4.72 \\
\hline 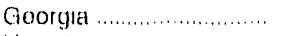 & 5.78 & 5.44 & 5.52 & 5.37 & 5.56 & 5.46 & 5.49 & 5.38 \\
\hline Hawaii ............................ & 11.64 & 11.34 & 11.63 & 10.75 & 10.76 & 11.51 & 11.94 & 12.36 \\
\hline 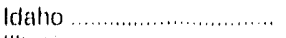 & 4.07 & 4.17 & 4,60 & 4.66 & 4.53 & 4.32 & 4.34 & 4.25 \\
\hline 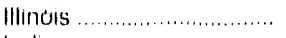 & 4.81 & 4.65 & 4.22 & 5.21 & 5.23 & 5.26 & 4.71 & 4.56 \\
\hline Indiana ........................... & 4.97 & 5.03 & 4.64 & 4.69 & 4.85 & 5.00 & 5.01 & 4.90 \\
\hline 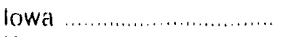 & 4.15 & 3.88 & 4.07 & 3.93 & 3.95 & 3.71 & 3.13 & 3.72 \\
\hline 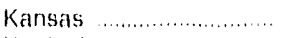 & 3.49 & 3.07 & 3.03 & 2.76 & 2.85 & 3.04 & 3.41 & 3.62 \\
\hline 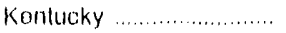 & 4.53 & 4.35 & 4.19 & 4.68 & 4.96 & 4.73 & 4.68 & 4.37 \\
\hline 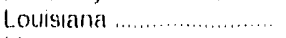 & 5.19 & 5.06 & 4.91 & 5.71 & 5.42 & 5.45 & 5.02 & 5.28 \\
\hline Maino .......................... & 6.97 & 6.20 & 6.35 & 6.47 & 6.43 & 6.25 & 6.57 & 7.50 \\
\hline Maryland ............................ & 5.46 & 5.39 & 5.09 & 5.35 & 5.28 & 5.23 & 5.07 & 6.11 \\
\hline Massalchusotls .............. & 6.31 & 5.91 & 6.13 & 5.29 & 1.78 & 4.89 & 5.27 & 6.79 \\
\hline Michigan ...................... & 4.66 & 4.91 & 4.91 & 5.46 & 5.48 & 5.07 & 4.75 & 4.56 \\
\hline 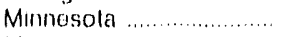 & 3.97 & 4.00 & 4.02 & 3.18 & 3.63 & 3.74 & 3.56 & $3.5 ?$ \\
\hline Mississippi ......................... & 4.38 & 4.82 & 5.18 & 3.93 & 3.98 & 3.93 & 4.20 & 4.14 \\
\hline Missouni ........................ & 4.60 & 4.30 & 4.19 & 4.60 & 4.54 & 4.49 & 4.45 & 4.60 \\
\hline 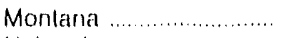 & 4.30 & 4.40 & 4.34 & 4.73 & 4.62 & 4.59 & 4.36 & 4.32 \\
\hline Nobraskal ........................ & 3.79 & 3.76 & 3.74 & 3.55 & 3.53 & 3.67 & 3.93 & 3.57 \\
\hline 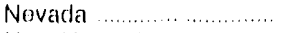 & 1.21 & 4.44 & 4.65 & 2.90 & 4.33 & 4.32 & 4.39 & 4.42 \\
\hline Now Hampshiro ............. & 6.83 & 6.18 & 5.60 & 6.39 & 6.50 & 6.41 & 6.23 & 7.29 \\
\hline Now Jorsoy ..................... & 5.46 & 5.26 & 5.26 & 5.00 & 5.05 & 5.11 & 5.10 & 5.41 \\
\hline Now Moxico .................. & 4.41 & 3.71 & 3.21 & 4.49 & 4.44 & 4.71 & 4.40 & 4.32 \\
\hline Now York ........................... & 5.59 & 5.73 & 5.29 & $4.8 B$ & 4.87 & 5.08 & ค 5.54 & 11 5.72 \\
\hline Norlh Garolina ............... & 4.69 & 5.18 & 4.93 & 4.63 & 4.63 & 4.68 & 4.67 & 4.48 \\
\hline Norlh Dakota ................... & 4.19 & 4.21 & 4.56 & 4.58 & 4.53 & 4.33 & 4.15 & 1.06 \\
\hline 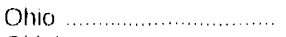 & 4.63 & 1.98 & 4.67 & 4.69 & 4.32 & 4.69 & 4.46 & 4.67 \\
\hline 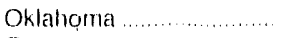 & 3.98 & 3.90 & 1.14 & 3.86 & 3.96 & 3.91 & 3.75 & 3.99 \\
\hline 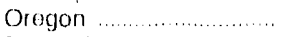 & 4.80 & 4.82 & 5.31 & 5.11 & 5.06 & 4.86 & 4.83 & $4.8 ?$ \\
\hline Pennsylvanial .................... & 5.99 & 5.25 & 5.01 & 6.35 & 6.28 & 6.39 & 6.09 & 5.96 \\
\hline Phodo Istand ................ & 6.23 & 6.60 & 5.83 & 5.20 & 4.85 & 7.09 & 5.65 & 5.75 \\
\hline South Carolina ............... & 5.59 & 5.63 & 5.63 & 5.13 & 5.12 & 5.25 & 5.24 & 5.67 \\
\hline South Dakola ............... & 4.14 & 3.90 & 4.11 & 4.04 & 4.39 & 4.27 & 4.01 & 3.90 \\
\hline 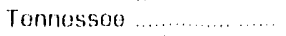 & 4.83 & 4.43 & 4.35 & 4.55 & 4.70 & 4.74 & 4.62 & 4.76 \\
\hline 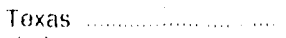 & 4.17 & 4.06 & 4.13 & 3.89 & A 3.68 & 3.71 & 4.03 & 4.10 \\
\hline Ulah $\ldots \ldots \ldots \ldots \ldots$ & 4.25 & 4.09 & 405 & 4.35 & 4.30 & 4.10 & 4.21 & 4.43 \\
\hline Vormonl ..... & 495 & 4.73 & 4.72 & 4.95 & 5.41 & 5.39 & 4.86 & 4.75 \\
\hline 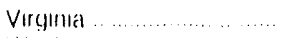 & 477 & 4.96 & 4.36 & 4.61 & 4.78 & 4.48 & 4.40 & 4.30 \\
\hline 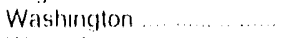 & 4.20 & 4.69 & 4.55 & 4.17 & 4.24 & 4.68 & 3.87 & 4.16 \\
\hline Wosi Virguna & 6.09 & 5.05 & 5.10 & 7.42 & 7.45 & 7.44 & 6.51 & 6.16 \\
\hline 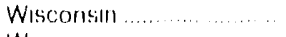 & 159 & 4.53 & 4.66 & 4.61 & 4.59 & 4.45 & 4.30 & 4.42 \\
\hline 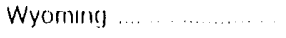 & 1.46 & $4.3 \%$ & 1.04 & 409 & 4.837 & 4.72 & 4.37 & 4.36 \\
\hline Tolal & 4.86 & 1.75 & $46 ?$ & 4.55 & 1.46 & 4.59 & na.6? & A $4.83 ?$ \\
\hline
\end{tabular}

Soe foolnotes at ond of lable 
Table 29. Average Price of Natural Gas Sold to Commercial Consumers by State (Continued)

(Dollars per Thousand Cublc Feet)

\begin{tabular}{|c|c|c|c|c|c|c|c|c|}
\hline 1 & $\begin{array}{l}\text { March } \\
1990\end{array}$ & $\begin{array}{c}\text { Fobrualy } \\
19000\end{array}$ & $\begin{array}{l}\text { Jamuary } \\
1990\end{array}$ & $\begin{array}{l}\text { Tolal } \\
\text { logen }\end{array}$ & $\begin{array}{c}\text { Dooomber } \\
19881\end{array}$ & $\begin{array}{l}\text { Nova'nber } \\
\left.19 t^{\circ}\right)\end{array}$ & $\begin{array}{l}\text { Octoben } \\
19(39)\end{array}$ & $\begin{array}{c}\text { Soplomituen } \\
\text { lenes }\end{array}$ \\
\hline Alabama ............... & 6.14 & 5.20 & 510 & 5.17 & 5.29 & 6.46 & 5.26 & 5.37 \\
\hline Nlasika $\ldots$ & 2.71 & 2.74 & $? .67$ & 2.57 & 2.53 & ?. 18 & 2.38 & 2.80 \\
\hline Anzonal & 4.78 & 4.80 & 4.83 & 1.76 & 4.75 & 4.80 & 4.85 & 4.74 \\
\hline Arkansas ..... ... .... & 4.39 & 4.34 & 425 & 4.39 & 1.21 & 4.45 & 4.78 & 4.92 \\
\hline Cabloma $\ldots \ldots \ldots \ldots$ & 5.64 & 5.64 & 5.56 & $4.8 \theta$ & 5.40 & 5.1 .1 & 4.35 & 4.30 \\
\hline Colorado $\ldots . . . . . . . . . . . .$. & 3.45 & 3.95 & 3.93 & 4.05 & 1.04 & 4.10 & 4.19 & 4.31 \\
\hline Comnuclicul ............ & 6.76 & 6.85 & 6.95 & G.08 & 6.70 & 6.30 & 5.88 & 5.34 \\
\hline Dolawalle & 5.51 & 5.36 & 5.32 & 9.39 & 535 & 5.45 & 5.66 & 5,18 \\
\hline Districe of Columbia ...... & 6.51 & 573 & 0.04 & 5.30 & 1.61 & 6.19 & 5.05 & 5.08 \\
\hline Flonda ................... & 4.94 & 5.23 & 5.083 & 4.835 & $\therefore 00$ & 4.837 & 4.76 & 4.69 \\
\hline 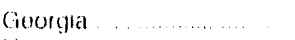 & 6.10 & 6.26 & 5.78 & 5.46 & 5.29 & 5.72 & 5.78 & 5.49 \\
\hline Hawail ........................ & 12.17 & 11.94 & 1173 & 11.44 & $115 ?$ & 11.84 & 1154 & 11.65 \\
\hline 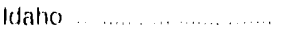 & 3.96 & 3.91 & 387 & 420 & $40 ?$ & 1.46 & 4.46 & 4.46 \\
\hline Illinols $\quad \ldots \ldots \ldots \ldots \ldots \ldots$ & 488 & 5.00 & 4.68 & 4.55 & 4.38 & 4.20 & 4.30 & 4.88 \\
\hline 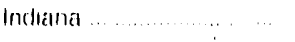 & 484 & 4.94 & 5.13 & 4.78 & 4.37 & $4.3 \%$ & 4.36 & 4.70 \\
\hline$\ldots \ldots$ & 307 & $43 \%$ & 4.63 & 3.91 & 4.14 & 3.83 & 3.64 & 186 \\
\hline Kansas . . . . & 386 & 3.91 & 3.87 & 3.11 & 379 & 324 & 3.1 .1 & 2.64 \\
\hline Kontucky & 4.39 & 450 & 4.54 & 1.35 & 4.23 & 4.39 & $1.4: 1$ & 1.60 \\
\hline 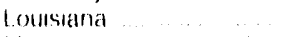 & 4.61 & 540 & 5.12 & 526 & 5.67 & 5.80 & 5.65 & 5.64 \\
\hline Manne & 7.40 & 7.42 & 6.46 & 6.30 & 6.43 & 6.57 & 6.47 & 6.68 \\
\hline Máryland ................... & 5.68 & 5.43 & 5.78 & 5.43 & 5.36 & 5,48 & 5.78 & 5.83 \\
\hline Massachusotts ............ & 7.06 & 7.13 & 6.2 .4 & 5.87 & 6.04 & 6.06 & 5.05 & 5.28 \\
\hline 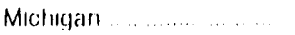 & $4.5 \%$ & 461 & 155 & 4.85 & 4.54 & 4.66 & 5.04 & 5.68 \\
\hline Minnosotal & 3.69 & 4.06 & Ifiti & 400 & 4.19 & 3.89 & 3.71 & $3.8: ?$ \\
\hline Mississinpu . . . . . . . . . . & 419 & $4.6 ?$ & 4.67 & 4.73 & 4.55 & 4.67 & 140 & $4(6)$ \\
\hline Misisoun _... . . . & 469 & 470 & 466 & 4.34 & 4.41 & 4.36 & 444 & $4.5 \%$ \\
\hline Monlana $\ldots$ & 423 & $4.2 \%$ & 4.19 & 4.36 & 4.26 & 4.31 & 4.28 & 4.36 \\
\hline Nobraska ... ......... & 381 & 379 & $42 \%$ & 3.17 & 3.39 & 3.74 & $3.6 f^{\prime}$ & 3.58 \\
\hline Nevada & 4.43 & 434 & $4.3 \%$ & $4.4 d$ & 4.57 & 4.40 & 444 & 4.28 \\
\hline Now Hamptihiro ..... & 730 & $71 \mathrm{~B}$ & 6.38 & 6.23 & 6.39 & 6.58 & 6.00 & 6.07 \\
\hline New Jursoy & 555 & $5.6 ?$ & 5.74 & 5.30 & 5.65 & 5.30 & 501 & 494 \\
\hline New Mexico , ......... & 4.37 & 444 & 4.39 & $37 \%$ & 3.68 & 3.53 & 4.30 & $40 \%$ \\
\hline New York ... ............ & $\mathrm{H} 5.70$ & 116.11 & $H 5,61$ & 562 & $5.18\}$ & $5.3 \%$ & 5.16 & 5.46 \\
\hline Norllı Carohma ............ & 463 & 4.67 & 4.89 & 5.14 & 5.16 & $4.9 ?$ & 4.90 & 606 \\
\hline Norlh Dakolal …...... & 4.19 & 117 & 4.16 & 4.19 & 405 & 4.12 & 4.61 & 393 \\
\hline Olmo $\quad \ldots \ldots \ldots$ & 404 & 496 & 493 & 4.00 & 473 & 4.810 & 4.83 & 4.92 \\
\hline Oklahoma $\ldots \ldots$ & 4.1:1 & 890 & 399 & 3.94 & 3.96 & 4.20 & 1.1\% & 4.11 \\
\hline Oroyon $\ldots \ldots \ldots$ & 4.86 & 489 & 4.89 & 4.80 & $A B \mid$ & 4.73 & $4 / 4$ & 481 \\
\hline Puncisiylvanla & 501 & 5.90 & 590 & 5.36 & 5,61 & 5.61 & 5.58 & $17 \%$ \\
\hline Rhodu Istand ........ & $6 ; 81$ & 6.74 & 6.63 & 6.41 & 6.42 & 5.83 & is $1 t$ & 700 \\
\hline South Ciaroliria & $56 !$ & 578 & 5.94 & 5.65 & 0.11 & $56 ?$ & $5.33^{\prime}$ & 43 \\
\hline South Dékola ........ & 391 & 415 & 440 & 3.09 & 414 & 381 & 380 & $39 y$ \\
\hline Tonnessoo ............ & 500 & 4.91 & $A B A$ & 449 & 4.12 & $4 \omega^{\prime}$ & 450 & 450 \\
\hline Tuxas & $45 \%$ & 4.54 & 466 & 411 & $4.3 \%$ & 4.26 & 4.00 & 4.16 \\
\hline 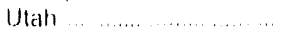 & 426 & 4.21 & 423 & 4.16 & 430 & 4.4 .1 & $4.6,4$ & $4: 30$ \\
\hline Vermont & 4.87 & $4.0 ! 0$ & $d ! 7$ & 474 & 4.96 & A. $(3)$ & 4.40 & 4.11 \\
\hline Virsunia $\ldots \ldots \ldots$ & 1.189 & 5.10 & 490 & 49.3 & 5.26 & 4.58 & 4.67 & 4.18 \\
\hline Washimglon $\ldots$ & 4.24 & 4.06 & $4: 34$ & 468 & 4.16 & A.tiis & $1.4 !$ & 567 \\
\hline Wost Virguna & Gicils? & 5.63 & $56 \%$ & 5.25 & $1,5,9$ & 5.59 & 5.43 & 620 \\
\hline Wisiconsin & 4.72 & 4.79 & 45,4 & 444 & 4.23 & 430 & 4.16 & 454 \\
\hline Wyoming $\quad \ldots \ldots$ & 4.30 & 431 & $1.3 H$ & 4.33 & 433 & $4: 36$ & 4.36 & 4.45 \\
\hline$\ldots \ldots \ldots$ & 14.93 & 5.06 & 4.99 & 4.71 & 481 & 471 & 1.61 & $A 6 \%$ \\
\hline
\end{tabular}

Seg lootnotes al and of lables 
Table 29. Average Price of Natural Gas Sold to Commercial Consumers by State (Continued) (Dollars per Thousand Cubic Feet)

\begin{tabular}{|c|c|c|c|c|c|c|c|c|}
\hline 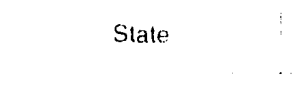 & $\begin{array}{c}\text { August } \\
1989\end{array}$ & $\begin{array}{c}\text { July } \\
1989\end{array}$ & $\begin{array}{l}\text { June } \\
1989\end{array}$ & $\begin{array}{l}\text { May } \\
1989\end{array}$ & $\begin{array}{l}\text { April } \\
1989\end{array}$ & $\begin{array}{c}\text { March } \\
1989\end{array}$ & $\begin{array}{l}\text { Fobruary } \\
1989\end{array}$ & $\begin{array}{c}\text { Jantuary } \\
19899\end{array}$ \\
\hline Alabama & 5.34 & 5.40 & 5.28 & 5.12 & 5.01 & 4.94 & 5.09 & 5.14 \\
\hline 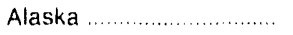 & 2.27 & 2.59 & 2.63 & 2.61 & $2.6^{\varsigma_{1}}$ & 2.68 & 2.74 & 2.65 \\
\hline 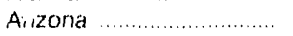 & 5.03 & 4.77 & 4.77 & 4.99 & 4.82 & 4.75 & 4.81 & 4.59 \\
\hline 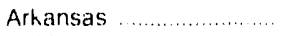 & 4.99 & 4.98 & 4.45 & 4.71 & 4.59 & 4.22 & 4.13 & 4.16 \\
\hline California ........................... & 4.57 & 4.63 & 4.07 & 4.28 & 5.16 & 5.27 & 5.25 & 5.28 \\
\hline 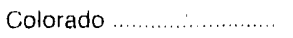 & 4.36 & 4.32 & 4.19 & 4.09 & 4.02 & 3.96 & 3.95 & 3.95 \\
\hline Connecticut ...................... & 4.98 & 5.26 & 5.61 & 5.54 & 6.15 & 6.04 & 6.29 & 6.31 \\
\hline Delaware & 5.46 & 5.65 & 5.61 & 5.50 & 5.43 & 5.33 & 5.27 & 5.33 \\
\hline District of Columbia ....... & 4.58 & 4.95 & 4.64 & 4.95 & 5.11 & 5.74 & 5.47 & 5.55 \\
\hline Florida ............................ & 4.85 & 4.79 & 4.86 & 4.89 & 4.85 & 4.83 & 4.83 & 4.88 \\
\hline 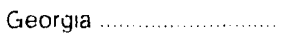 & 5.55 & 5.62 & 5.55 & 5.49 & 5.38 & 5.53 & 5.46 & 5.27 \\
\hline 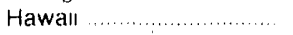 & 11.72 & 11.91 & 12.05 & 11.46 & 11.55 & 11.09 & 10.59 & 10.43 \\
\hline 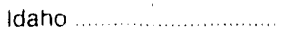 & 4.57 & 4.50 & 4.49 & 4.49 & 4.45 & 4.11 & 4.02 & 3.99 \\
\hline 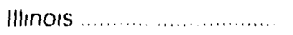 & 5.19 & 5.10 & 4.84 & 4.62 & 4.61 & 4.77 & 4.66 & 4.42 \\
\hline Indiana ........................... & 4.73 & 4.83 & 4.98 & 5.30 & 5.13 & 5.03 & 4.89 & 5.11 \\
\hline 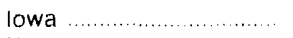 & 3.90 & 3.83 & 3.71 & 3.62 & 3.61 & 3.75 & 3.99 & 4.17 \\
\hline 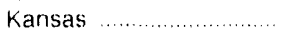 & 2.62 & 2.83 & 2.69 & 2.84 & 2.96 & 3.32 & 3.34 & 3.27 \\
\hline Kenlucky ......................... & 4.76 & 4.63 & 4.48 & 4.48 & 4.30 & 4.32 & 4.28 & 4.30 \\
\hline 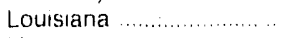 & 5.61 & 5.26 & 5.47 & 4.87 & 5.01 & 4.72 & 4.96 & 5.18 \\
\hline 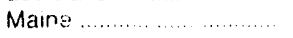 & 6.43 & 6.56 & 6.46 & 6.28 & 6.25 & 6.12 & 6.11 & 6.11 \\
\hline 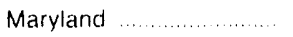 & 5.70 & 5.85 & 5.52 & 5.42 & 5.47 & 5.14 & 5.44 & 5.34 \\
\hline Massachusetts ............... & 4.68 & 5.28 & 5.26 & 5.37 & 6.04 & 6.16 & 6.23 & 6.21 \\
\hline 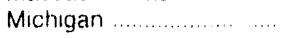 & 5.99 & 5.75 & 5.31 & 4.90 & 4.79 & 4.81 & 4.84 & 4.87 \\
\hline 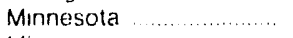 & 3.88 & 3.93 & 3.92 & 3.79 & 3.80 & 3.88 & 4.05 & 4.26 \\
\hline Mississippi ......................... & 4.57 & 4.66 & 4.63 & 4.93 & 4.72 & 4.80 & 4.94 & 4.90 \\
\hline 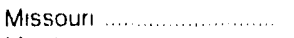 & 4.74 & 479 & 4.64 & 4.47 & 4.22 & 4.22 & 4.25 & 4.21 \\
\hline Montana ......................... & 4.36 & 4.46 & 4.45 & 4.43 & 4.41 & 4.37 & 4.40 & 4.39 \\
\hline 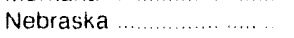 & 3.59 & 3.49 & 3.78 & 3.73 & 3.67 & 3.74 & 3.95 & 4.05 \\
\hline 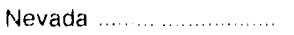 & 4.37 & 4.47 & 4.60 & 4.57 & 4.63 & 4.58 & 4.29 & 4.27 \\
\hline New Hampshire .............. & 6.18 & 6.16 & 5.92 & 570 & 6.31 & 6.25 & 6.28 & 6.20 \\
\hline New Jersey ..................... & 4.97 & 4.97 & 5.08 & 5.05 & 5.23 & 5.40 & 5.43 & 5.28 \\
\hline New Mexicio .................... & 3.81 & 4.20 & 3.78 & 3.50 & 3.83 & 3.52 & 3.64 & 3.78 \\
\hline 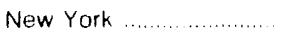 & 5.55 & 5.51 & 5.54 & 5.70 & 5.86 & 5.95 & 5.7 .0 & 5.66 \\
\hline North Caroilna ................ & 5.05 & 5.04 & 4.95 & 4.95 & 5.31 & 5.28 & 5.25 & 5.19 \\
\hline 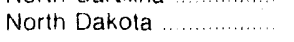 & 4.54 & 4.46 & 4.32 & 4.31 & 4.18 & 4.15 & 4.18 & 4.21 \\
\hline 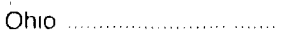 & 5.25 & 5.15 & 4.98 & 4.70 & 5.01 & 4.86 & 5.05 & 5.03 \\
\hline Oklahoma & 465 & 4.26 & 4.18 & 4.08 & 4.12 & 3.86 & $3 . \% 1$ & 3.75 \\
\hline Oregon & 4.85 & 4.79 & 476 & 470 & 4.72 & 4.71 & 4.73 & 514 \\
\hline Pennsylvania .................. & 5.64 & 5.67 & 5.37 & 5.26 & 5.21 & 5.20 & 5.20 & 5.16 \\
\hline Rhode Island ................ & 7.11 & 6.91 & 7.31 & 6.63 & 6.32 & 6.51 & 6.77 & 6.38 \\
\hline South Carolına .............. & 5.23 & 5.38 & 4.85 & 5.81 & 5.51 & 5.82 & 5.93 & 5.73 \\
\hline South Danota & 4.15 & 4.10 & 4.05 & 3.96 & 3.89 & 3.87 & 3.94 & 4.19 \\
\hline 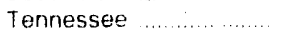 & 4.54 & 451 & 4.47 & 4.20 & 4.41 & 4.35 & 4.51 & 4.46 \\
\hline Texas & 3.80 & 374 & 3.97 & 3.96 & 3.93 & 4.12 & 4.30 & 4.25 \\
\hline 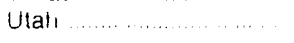 & 4.23 & 4.13 & 4.00 & 4.17 & 4.30 & 4.13 & 4.03 & 4.03 \\
\hline 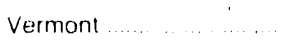 & 4.92 & 5.01 & 5.13 & 4.99 & 4.73 & 4.70 & 4.88 & 434 \\
\hline 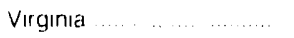 & 4.59 & 4.52 & 4.34 & 4.92 & 4.81 & 5.04 & 5.23 & 5.17 \\
\hline 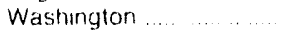 & 466 & 4.77 & 4.91 & 4.83 & 4.70 & 4.51 & 4.74 & 4.67 \\
\hline West Virgirila & 6.21 & 6.40 & 6.10 & 5.31 & 5.03 & 4.87 & 4.81 & 4.82 \\
\hline Wisconsin $\ldots \ldots \ldots$ & 4.74 & 4.54 & 4.40 & 4.23 & 4.46 & 4.50 & 4.64 & 4.59 \\
\hline Wyoming $\ldots \ldots$ & 4.45 & 4.40 & 4.31 & 4.35 & 4.31 & 4.30 & 4.31 & 4.31 \\
\hline$\ldots \ldots \ldots \ldots \ldots \ldots \ldots$ & 461 & 465 & 4.57 & 464 & 4.77 & 4.79 & 480 & 1.81 \\
\hline
\end{tabular}

F Revised Data

Notes and Sources See the last page of this section 
Table 30. Average Price of Natural Gas Sold to Industrial Consumers by State

\section{(Doliars per Thousand Cubic Feet)}

\begin{tabular}{|c|c|c|c|c|c|c|c|c|}
\hline Sitate? & $\begin{array}{l}\text { YTD } \\
\text { t990 }\end{array}$ & $\begin{array}{l}Y T D \\
19899\end{array}$ & $\begin{array}{l}\text { YTD } \\
19888\end{array}$ & $\begin{array}{c}\text { August } \\
1990\end{array}$ & $\begin{array}{l}\text { July } \\
1990\end{array}$ & $\begin{array}{l}\text { June } \\
1990\end{array}$ & $\begin{array}{c}\text { May } \\
1990\end{array}$ & $\begin{array}{l}\text { April } \\
1990\end{array}$ \\
\hline Alatbarma & 304 & 2.96 & 3.01 & 274 & 2.76 & 2.85 & 3.01 & 3.12 \\
\hline Alaski. & 1.07 & 1.11 & 1.04 & 1.08 & 1.03 & 95 & 1.18 & 1.15 \\
\hline Arzona & $38 \%$ & 384 & 3.76 & 4.30 & 4.30 & 4.23 & 4.11 & 3.70 \\
\hline Arkanlsct: & 300 & 309 & 307 & 2.75 & 2.81 & 2.81 & ?. 84 & 2.88 \\
\hline Callforma & 390 & 389 & 371 & 4.07 & 3.65 & 3.64 & 3.67 & 3.65 \\
\hline (Cotoratio). & 2?28 & 2. 50 & 3.51 & 212 & 2.17 & 2.10 & 2.16 & 2.28 \\
\hline Comnecticu: & $4.7 ?$ & 452 & 408 & 3.83 & 3.84 & 3.96 & 4.01 & 4.70 \\
\hline Delawate: & 343 & 3.46 & 3.18 & 3.16 & 3.32 & 308 & 3.29 & 3.04 \\
\hline Distroct of Columbat & & & $\ldots . .$. & $\ldots$ & $\ldots$ & $\ldots$ & $\ldots$ & $\ldots$ \\
\hline Honda & 315 & 309 & 2.83 & 324 & 281 & 291 & 2.95 & 2.97 \\
\hline Gerenches. & $3 i, 1$ & 367 & 3 titi & 2.99 & 309 & 3.40 & 3.33 & 3.56 \\
\hline Hawall & & .... & $\ldots$ & $\ldots$ & $\ldots$ & $\ldots$ & $\ldots$ & $\ldots$ \\
\hline Idaho. & 254 & 2.35 & 412 & 2.955 & 2.97 & 2.87 & 2.83 & 2.84 \\
\hline Illinous & 415 & 386 & 341 & 3.44 & 3.85 & 4.33 & 4.16 & 4.05 \\
\hline Indiatial & 386 & $3 \% 4$ & 3.73 & 3.07 & 309 & 3.25 & 3.49 & 3.95 \\
\hline lowat. & 275 & 2.53 & 3.19 & 228 & 2.31 & 2.29 & 2.44 & 2.61 \\
\hline Kantsats & 283 & 3011 & 2.37 & 2.54 & 2.57 & 3.02 & 2.32 & 2.49 \\
\hline Kentucky & 390 & $3 \pi$ & 343 & 3.63 & 3.89 & 3.68 & 3.75 & 3.93 \\
\hline L nusiana & 199 & 199 & $1.9 i$ & 1.67 & 1.71 & 1.76 & 1.86 & 1.82 \\
\hline Manne . . & $\therefore 16$ & 450 & 466 & 497 & 4.10 & 4.05 & 4.22 & 5.64 \\
\hline Marylan!n! & 469 & $47 H$ & 4.24 & 4.51 & 4.14 & 4.55 & 4.40 & 4.41 \\
\hline Masseatfubetts, & 40 & 411 & 1.24 & 3.69 & 298 & 291 & 3.77 & 4.12 \\
\hline Methelatn & 4.20 & 131 & 4.26 & $4.2 \%$ & 4.483 & 4.04 & 4.17 & 4.11 \\
\hline Munbresolata & 300 & 2.83 & 2.77 & 2.58 & 2.72 & 2666 & 2.66 & 2.77 \\
\hline Mussissip) & 248 & 253 & 251 & 223 & 232 & 2.24 & 2.34 & 2.35 \\
\hline Mrsocurn & 4.7 & $41 \%$ & 3.80 & 4.16 & 3.88 & 4.25 & 4.69 & 4.41 \\
\hline Montania & 306 & $i^{2} .44$ & 300 & 254 & 2.70 & 2.88 & 292 & 2.93 \\
\hline Netuatitis & 301 & 293 & $2.8 \%$ & 2.66 & 274 & 2.67 & 2.47 & 2.88 \\
\hline Nevadea & $4: 31$ & 5.05 & 389 & 5,58 & 4.36 & 4.12 & 5.05 & 4.11 \\
\hline New Hathps:hure: & $43 i^{2}$ & 410 & 3.71 & 3.75 & 3.61 & 3.37 & 3.27 & 4.11 \\
\hline Now lens:y & 1.19 & 40, & 3.87 & 3.46 & 3.46 & 3.53 & 3.64 & 4.03 \\
\hline Now Mexter & $3 t i b$ & 36169 & 3.27 & 3.44 & 3.39 & 3.38 & 3.65 & 3.87 \\
\hline Now York & $4 \%$ & $49 \%$ & $1.4 f$ & 3.86 & 4.16 & 4.36 & 4.24 & 4.87 \\
\hline North Garoluna & 336 & 3.69 & 359 & 3.07 & 2.94 & 312 & 3.19 & 3.21 \\
\hline North Dekota & 3.17 & 338 & 3.30 & 2.97 & 3.00 & 297 & 2.99 & 309 \\
\hline Ohuo & 397 & $42^{5}$ & 4.03 & 373 & 3.66 & 3.66 & 3.70 & 3.91 \\
\hline OWathoma & 170 & $1 \%$ & 189 & 1.75 & 1.62 & 1.71 & 1.71 & 1.69 \\
\hline Crryon & 330 & 304 & 372 & 293 & 2.80 & 289 & 3.40 & 3.31 \\
\hline 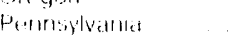 & 423 & 386 & 3.68 & 4.03 & 4.13 & 3.91 & 4.23 & 4.22 \\
\hline Rhosde Islatios & 524 & 5,183 & 175 & 454 & 503 & H 4.49 & 4.43 & 4.77 \\
\hline South Garrsthat & $3 \ln$ & 343 & 312 & 253 & 2.43 & 2.86 & 3.02 & $3.16 \mathrm{j}$ \\
\hline Sonuth bakotet & $32 \%$ & $3: 0$ & 3.15 & $31 \%$ & 303 & 292 & 297 & 3.07 \\
\hline Teromestiben & 331 & $33 \mathrm{i}^{2}$ & $32 \%$ & 295 & 2.91 & 306 & 3.01 & 3.27 \\
\hline Texas & c\%? & 226 & 216 & 1.83 & 1.92 & 193 & 1.99 & 2.09 \\
\hline Utah & 363 & 328 & 3.04 & 3.26 & 3.28 & 3.24 & 3.28 & 3.48 \\
\hline Vermunit & $\therefore y^{2}$ & ?49 & $30 \%$ & 2.28 & 229 & 264 & $2.7 \%$ & 2.95 \\
\hline Vrrejertiat & $3 \%$ & $390^{\circ}$ & 314 & 349 & $36 t$ & $34 ?$ & 3.34 & 364 \\
\hline Wastustem & $\because 0$ & $\because 4 \mathrm{~F}$ & i"stis. & $25 ?$ & 2.58 & 2.47 & 2.43 & 2.63 \\
\hline West viguturs & $3 \cdot n$ & $\therefore 4$ & $\therefore 84$ & 301 & 3.07 & 307 & 317 & 3.22 \\
\hline Wheconstit & 36,6 & 3830 & 104 & 3.25 & 347 & 3.183 & 3.34 & 339 \\
\hline Wroming & 300 & $3: 4$ & 334 & 355 & 3.27 & 315 & 3.13 & 3.18 \\
\hline |rital & 293 & $\because 39$ & 294 & $25 \%$ & 247 & 2.55 & 2.67 & 284 \\
\hline
\end{tabular}


Table 30. Average Price of Natural Gas Sold to Industrial Consumers by State (Continued) (Dollars per Thousand Cubic Feet)

\begin{tabular}{|c|c|c|c|c|c|c|c|c|}
\hline State & $\begin{array}{c}\text { March } \\
1990\end{array}$ & $\begin{array}{c}\text { February } \\
1990\end{array}$ & $\begin{array}{c}\text { January } \\
1990\end{array}$ & $\begin{array}{l}\text { Total } \\
1989\end{array}$ & $\begin{array}{c}\text { December } \\
1989\end{array}$ & $\begin{array}{c}\text { November } \\
1989\end{array}$ & $\begin{array}{c}\text { October } \\
1989\end{array}$ & $\begin{array}{c}\text { Seplember } \\
1989\end{array}$ \\
\hline 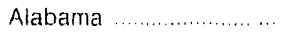 & 3.05 & 3.24 & 3.45 & 3.02 & 3.55 & 3.00 & 2.98 & 2.94 \\
\hline 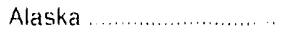 & 1.06 & 1.08 & 1.08 & 1.08 & 1.06 & 1.04 & .97 & .99 \\
\hline 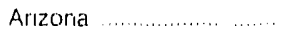 & 3.48 & 3.59 & 3.66 & 3.67 & 4.13 & 3.07 & 3.06 & 3.35 \\
\hline 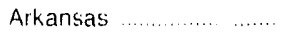 & 3.17 & 3.22 & 3.29 & 3.09 & 3.12 & 3.12 & 3.06 & 3.02 \\
\hline Calıfornia $\ldots \ldots \ldots \ldots \ldots \ldots$ & 4.30 & 4.55 & 3.84 & 3.75 & 3.88 & 4.72 & 3.07 & 3.35 \\
\hline 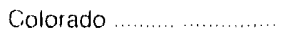 & 2.40 & 2.41 & 2.41 & 2.53 & 2.65 & 2.56 & 2.59 & 2.53 \\
\hline Connecticut ........................ & 5.27 & 5.59 & 6.00 & 4.51 & 5.55 & 4.41 & 3.90 & 3.91 \\
\hline Delaware & 3.53 & 3.82 & 400 & 3.45 & 3.70 & 3.23 & 3.37 & 3.39 \\
\hline District of Columbia ...... & -- & - & $\cdots$ & - & -- & -- & $\cdots$ & -- \\
\hline Florida $\ldots . . . . . . . . . . . \ldots \ldots \ldots$ & 3.06 & 3.48 & 3.65 & 3.13 & 3.40 & 3.22 & 3.06 & 3.10 \\
\hline 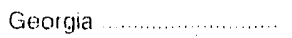 & 3.77 & 4.21 & 3.70 & 3.73 & 4.13 & 3.74 & 3.75 & 3.55 \\
\hline 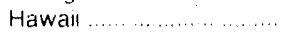 & -. & - & -- & -. & - & -. & -- & $\ldots$ \\
\hline 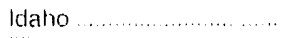 & 2.83 & 2.83 & 2.43 & 2.92 & 2.73 & 2.76 & 4.12 & 3.27 \\
\hline 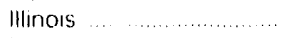 & 4.19 & 4.17 & 4.34 & 3.73 & 370 & 3.49 & 3.29 & 3.41 \\
\hline Indiana $\ldots \ldots \ldots \ldots \ldots . . . . . . . . . .$. & 4.19 & 4.08 & 4.48 & 3.89 & 4.04 & 3.69 & 3.59 & 3.64 \\
\hline lowa $\ldots . . . \ldots \ldots \ldots \ldots$ & 2.77 & 309 & 3.49 & 2.54 & 3.25 & 2.58 & 2.25 & 2.19 \\
\hline 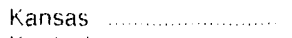 & 2.85 & 3.00 & 3.26 & 3.06 & 3.27 & $3.0 i$ & 3.44 & 2.75 \\
\hline Kentucky & 3.95 & 3.93 & 4.01 & 3.69 & 3.47 & 3.56 & 3.41 & 3.64 \\
\hline 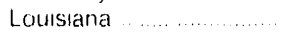 & 2.20 & 2.56 & 2.51 & 1.97 & 2.12 & 1.90 & 1.81 & 1.87 \\
\hline 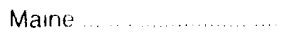 & 6.35 & 6.89 & 5.58 & 4.57 & 5.68 & 4.78 & 4.11 & 4.23 \\
\hline Maryland ......... & 4.53 & 4.59 & 4.86 & 4.81 & 5.14 & 4.78 & 4.72 & 4.66 \\
\hline Massachuselts .............. & 5.39 & 5.99 & 5.42 & 4.07 & 5.33 & 4.18 & 3.31 & 3.35 \\
\hline Michigan ..................... & 4.15 & 4.22 & 4.27 & 4.22 & 3.83 & 3.93 & 4.16 & 4.49 \\
\hline Minnesota ..................... & 2.84 & 3.25 & 3.67 & 2.86 & 3.26 & 2.85 & 2.61 & 2.69 \\
\hline Mississippi _................... & 2.51 & 2.78 & 2.99 & 2.54 & 2.87 & 2.60 & 2.45 & 2.33 \\
\hline Missouri …................. & 4.57 & 4.42 & 4.62 & 4.13 & 4.33 & 4.07 & 4.11 & 4.01 \\
\hline 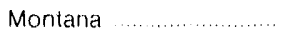 & 3.11 & 3.45 & 3.63 & 2.98 & 3.76 & 3.84 & 2.68 & 2.55 \\
\hline Nebraska .................... & 3.03 & 3.20 & 3.60 & 292 & 3.19 & 284 & 2.73 & 2.72 \\
\hline 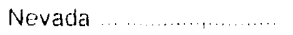 & 380 & 4.37 & 4.04 & 4.98 & 4.48 & 4.98 & .33 & 6.15 \\
\hline New Hampshire .............. & 5.92 & 6.09 & 5.54 & 4.20 & 6.04 & 5.14 & 3.64 & 3.27 \\
\hline New Jersey .................. & 4.42 & 4.89 & 5.03 & 3.98 & 4.40 & 3.89 & 3.45 & 3.38 \\
\hline New Mexico ...................... & 4.18 & 4.27 & 4.34 & 3.12 & 3.20 & 3.33 & 3.15 & 3.01 \\
\hline 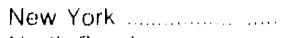 & 5.26 & 3.22 & 4.94 & 4.83 & 5.10 & 4,61 & 4.31 & 3.99 \\
\hline North Carolina $\ldots . . . . . . . . .$. & 3.45 & 3.53 & 3.72 & 3.64 & 4.18 & 3.63 & 3.59 & 3.51 \\
\hline North Dakota ................. & 3.25 & 4.13 & 3.67 & 3.55 & 5.13 & 3.30 & 3.09 & 3.18 \\
\hline 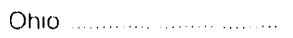 & 3.88 & 4.28 & 4.22 & 4.19 & 4.21 & 4.17 & 3.97 & 3.84 \\
\hline 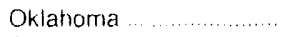 & 1.83 & 1.48 & 1.81 & 1.97 & 2.11 & 1.89 & 2.15 & 2.04 \\
\hline Oregon $\ldots . . . . . . . . . . . . . . . . . .$. & 3.45 & 3.71 & 3.66 & 3.52 & 3.67 & 3.41 & 3.16 & 3.22 \\
\hline Dennsylvania ....................... & 4.18 & 439 & 4.41 & 3.90 & 4.1, & 3.82 & 3.82 & 3.94 \\
\hline 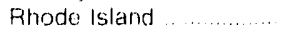 & 5.84 & 6.23 & 6.36 & 5.16 & 6.17 & 5.70 & 4.39 & 4.35 \\
\hline South Carolina ............. & 3.33 & 3.62 & 3.93 & 3.46 & 4.17 & 3.54 & 3.29 & 3.22 \\
\hline South Dakola ............. & 3.19 & 3.50 & 3.82 & 3.09 & 3.33 & 3.02 & 2.87 & 2.96 \\
\hline Tennessee $\ldots . . . . . . . . . . . .$. & 3.33 & 3.70 & 3.94 & 3.37 & 3.71 & 3.39 & 3.21 & 3.23 \\
\hline 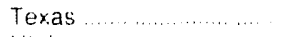 & 2.09 & 2.64 & 3.13 & 2.26 & 2.68 & 2.26 & 2.08 & 2.02 \\
\hline 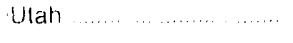 & 3.48 & 4.93 & 3.45 & 3.30 & 3.38 & 3.40 & 3.41 & 3.18 \\
\hline Vermont $\ldots \ldots \ldots \ldots \ldots$ & 3.43 & 4.02 & $3 j 3$ & 3.01 & 3.35 & 3.09 & 2.87 & 3.00 \\
\hline 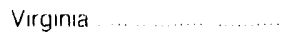 & 3.74 & 4.25 & 4.33 & 3.91 & 4.17 & 3.68 & 4.22 & 3.48 \\
\hline 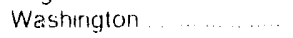 & 2.59 & 2.93 & 3.12 & 2.93 & 3.03 & 3.06 & 2.75 & 2.70 \\
\hline Wesi Virgiria & 3.49 & 3.65 & 2.67 & 2.92 & 3.62 & 301 & 2.72 & 2.82 \\
\hline Wiscorisin & 381 & 3.97 & 394 & 3.73 & 3.5 .3 & 3.84 & 3.58 & 3.56 \\
\hline Wyoming $\ldots \ldots \ldots \ldots$ & 2.50 & 3.24 & $\therefore 15$ & 3.23 & $30 \%$ & 3.17 & 3.11 & 2.91 \\
\hline Total & 3.08 & 3.40 & 352 & 2.97 & 3.32 & 2.98 & 2.75 & 2.67 \\
\hline
\end{tabular}

See footnotes at end of table 
Table 30. Average Price of Natural Gas Sold to Industrial Consumers by State (Continued) (Dollars per Thousand Cubic Feet)

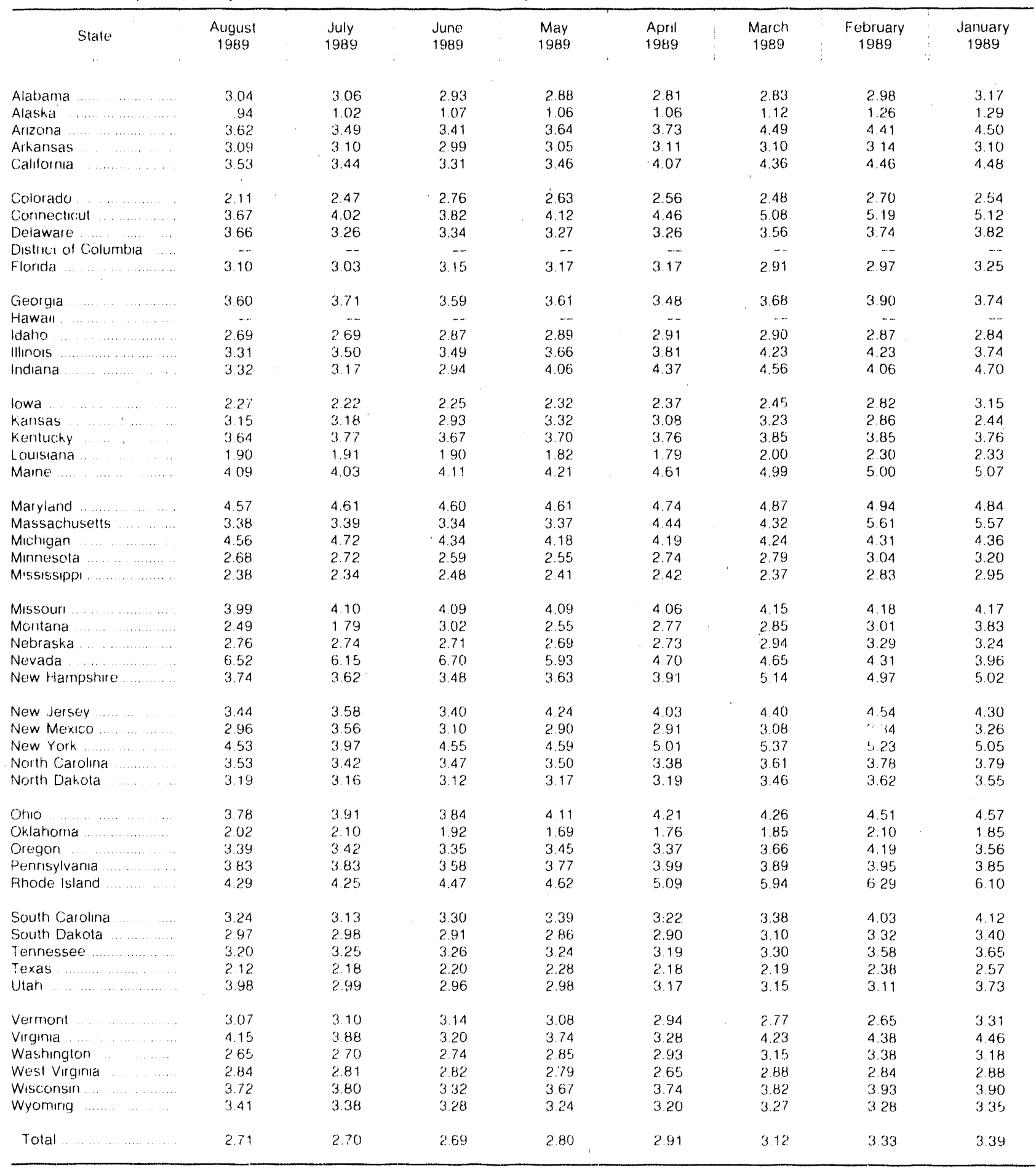

H Revised Data.

Notes and Sources See the last page of this section 
Table 31. Average Price of Natural Gas Delivered to Electric Utility Consumers by State (Dollars per Thousand Cubic Feet)

\begin{tabular}{|c|c|c|c|c|c|c|c|c|}
\hline State & $\begin{array}{l}\text { July } \\
1990\end{array}$ & $\begin{array}{l}\text { June } \\
1990\end{array}$ & $\begin{array}{l}\text { May } \\
1990\end{array}$ & $\begin{array}{l}\text { April } \\
1990\end{array}$ & $\begin{array}{c}\text { March } \\
1990\end{array}$ & $\begin{array}{c}\text { February } \\
1990\end{array}$ & $\begin{array}{c}\text { January } \\
1990\end{array}$ & $\begin{array}{l}\text { Total } \\
1989\end{array}$ \\
\hline Alabama $\ldots \ldots \ldots \ldots \ldots$ & 1.97 & 2.03 & 1.99 & 1.97 & 2.16 & 2.65 & 3.11 & 2.27 \\
\hline 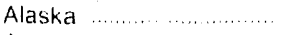 & NA & NA & -- & $\ldots$ & $\ldots$ & -. & $\ldots$ & 1.36 \\
\hline Arizona & 228 & 2.30 & 2.30 & 2.34 & 2.25 & 2.84 & 3.30 & 2.32 \\
\hline Arkansas ..................... & 1.52 & 1.45 & 1.51 & 1.37 & 1.33 & 1.94 & 1.93 & 1.69 \\
\hline California ................. & 2.82 & 2.93 & 3.10 & 3.06 & 3.02 & 3.66 & 3.82 & 3.04 \\
\hline Colorado .......................... & 2.21 & 2.22 & 2.13 & 2.13 & 2.12 & 2.30 & 2.37 & 2.23 \\
\hline Connecticut ...................... & 2.04 & 2.13 & 2.19 & -- & -. & 6.51 & 6.27 & 2.59 \\
\hline Delaware ......................... & 2.29 & 2.13 & 2.17 & 3.15 & 2.95 & 3.51 & 3.48 & 2.73 \\
\hline District of Colurnbia ....... & -- & -- & -- & $\cdots$ & -- & -- & $\ldots$ & $\cdots$ \\
\hline Florida & 2.23 & 1.91 & 2.19 & 2.10 & 2.36 & 2.63 & 3.29 & 2.49 \\
\hline Georgla .......................... & 2.89 & 3.29 & 3.34 & 3.52 & 3.73 & 3.80 & 3.75 & 3.23 \\
\hline 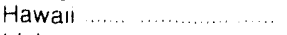 & -. & -- & -. & -. & -. & -- & -. & -. \\
\hline Idaho & -- & -. & -. & -- & -- & -. & -. & $\cdots$ \\
\hline Illinols .......................... & 2.77 & 2.47 & 2.77 & 2.99 & 3.39 & 3.90 & 3.48 & 3.32 \\
\hline Indiana & 2.53 & 2.29 & 2.35 & 2.46 & 2.61 & 2.82 & 3.25 & 2.70 \\
\hline 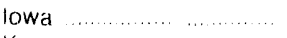 & 2.96 & 2.68 & 2.09 & 3.00 & 3.46 & 3.71 & 4.36 & 2.69 \\
\hline 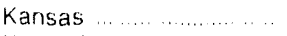 & 1.60 & 1.62 & 1.65 & 1.97 & 1.98 & 2.23 & 2.33 & 1.92 \\
\hline …….............. & 2.49 & 3.12 & 3.19 & 3.14 & 3.65 & 3.35 & 3.37 & 2.73 \\
\hline Loulsiana ........................ & 1.72 & 1.72 & 1.65 & 1.62 & 1.64 & 1.73 & 2.01 & 1.78 \\
\hline Marne & -.. & $\cdots$ & -- & -- & -- & -. & -.. & .. \\
\hline Maryland …....... & 2.27 & 2.34 & 2.53 & 2.75 & 3.00 & 3.38 & 2.84 & 2.72 \\
\hline Massachusetts ….............. & 2.03 & 2.29 & 2.29 & 2.30 & 2.36 & 2.75 & 5.13 & 2.49 \\
\hline Michigan ......................... & 65 & 51 & .50 & 37 & .34 & .49 & .31 & .19 \\
\hline Mirnesota & 1.75 & 1.95 & 1.81 & 1.90 & 1.92 & 2.46 & 3.12 & 2.16 \\
\hline Mississippi ....................... & 1.69 & 1.76 & 1.76 & 1.74 & 1.82 & 2.14 & 2.52 & 1.88 \\
\hline 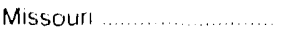 & 2.00 & 2.26 & 2.32 & 2.79 & 2.48 & 2.79 & 3.19 & 2.50 \\
\hline 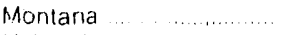 & 3.20 & 5.18 & 1.60 & 1.27 & 1.51 & 3.16 & 1.40 & 1.38 \\
\hline Nebraska. & 2.13 & 2.12 & 1.67 & 1.66 & 1.74 & 2.68 & 3.35 & 2.26 \\
\hline 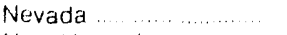 & 2.09 & 2.05 & 1.80 & 1.72 & $1: 73$ & 2.76 & 3.18 & 2.17 \\
\hline New Hampshire .............. & - & -- & $\cdots$ & -- & - & $-\cdots$ & -- & 3.16 \\
\hline New Jersey. & 2.20 & 2.13 & 2.29 & 2.19 & 2.59 & 3.02 & 3.78 & 2.54 \\
\hline New Mexico .................. & 1.90 & 1.91 & 1.84 & 1.89 & 1.90 & 2.14 & 2.71 & 2.20 \\
\hline New York ....... & 2.05 & 2.11 & 2.21 & 2.31 & 2.50 & 2.95 & 3.47 & 2.42 \\
\hline North Carolina ............. & NA & NA & $-\cdots$ & -- & - & -- & -- & 3.57 \\
\hline North Dakota ................... & NA & -. & $\cdots$ & -. & -- & -. & -. & 4.61 \\
\hline Oho & 1.97 & 3.21 & 3.28 & 4.00 & 3.67 & 4.10 & 4.14 & 3.15 \\
\hline Oklahoma ................... & 301 & 3.11 & 3.06 & 3.61 & 3.52 & 3.62 & 3.34 & 3.05 \\
\hline Dregori & NA & $-\ldots$ & -- & -. & -- & -- & -. & -- \\
\hline Pennsylvania. & NA & 3.02 & 2.95 & 2.92 & 2.86 & 3.47 & 3.34 & 3.58 \\
\hline Rhode Island & 1.99 & 2.02 & 2.07 & 2.22 & 2.35 & $\cdots$ & .. & 2.49 \\
\hline South Caroliria. & 1.69 & 1.83 & 3.95 & 4.70 & 4.53 & 4.49 & 4.37 & 2.27 \\
\hline South Dakota ... & NA & NA & $\cdots$ & -- & -- & -- & -. & 2.62 \\
\hline 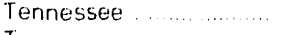 & NA & NA & $\ldots$ & -- & -- & $\cdots$ & -- & 2.83 \\
\hline 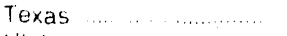 & 2.10 & 2.00 & 1.96 & 1.96 & 2.12 & 2.46 & 2.76 & 2.23 \\
\hline 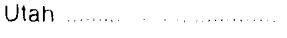 & NA & NA & … & 5.04 & -- & -. & -- & 3.38 \\
\hline Vermont & NA & NA & $\ldots$ & -- & ... & -. & -. & -. \\
\hline Virgınıa ........ & 2.06 & 1.68 & -. & $\ldots$ & -. & $\cdots$ & -. & 2.57 \\
\hline Washington & NA & NA & .. & -- & $\cdots$ & -- & ... & 5.19 \\
\hline West Virginia & 3.31 & 658 & 6.45 & 6.63 & 5.99 & 4.82 & 4.76 & 4.59 \\
\hline Wisconsin & 271 & 295 & 295 & 2.81 & 2.83 & 3.40 & 3.64 & 3.11 \\
\hline Wyoming $\ldots . . . . . . .$. & 3.26 & 3.28 & 323 & 324 & 3.93 & 3.92 & 3.88 & 3.61 \\
\hline Total. & 2.22 & 2.16 & 2.19 & 2.29 & 2.37 & 2.76 & 3.01 & 2.43 \\
\hline
\end{tabular}

See footnotes at end of table. 
Table 31. Average Price of Natural Gas Delivered to Electric Utility ${ }^{a}$ Consumers by State (Continued) (Dollars per Thousand Cubic Feet)

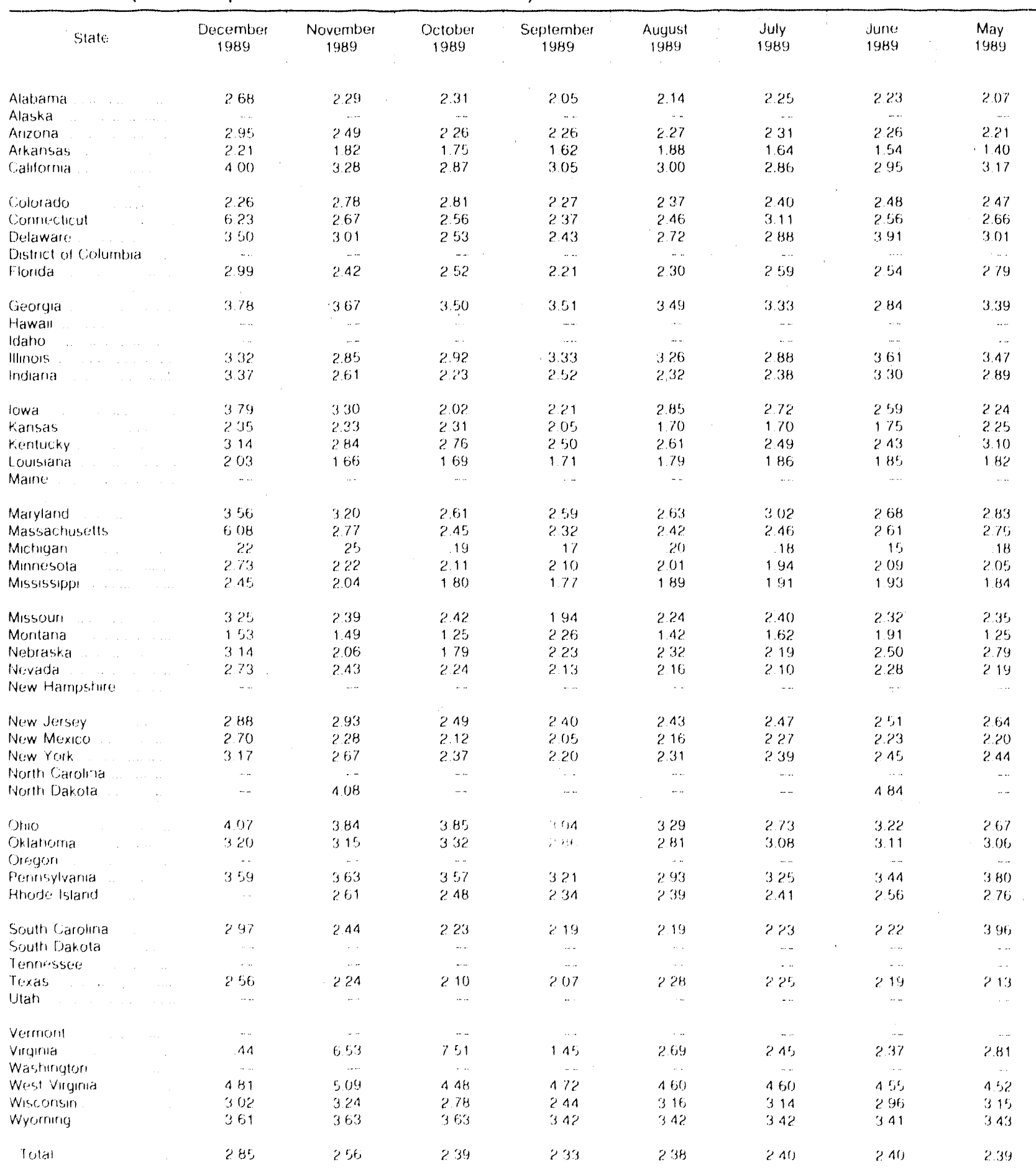

Seretootroles at and of latse: 
Table 31. Average Price of Natural Gas Delivered to Electric Utility ${ }^{a}$ Consumers by State (Continued) (Dollars per Thousand Cubic Feet)

\begin{tabular}{|c|c|c|c|c|c|c|c|c|}
\hline State & $\begin{array}{l}\text { April } \\
1989\end{array}$ & $\begin{array}{c}\text { March } \\
1989\end{array}$ & $\begin{array}{c}\text { February } \\
1989\end{array}$ & $\begin{array}{c}\text { January } \\
1989\end{array}$ & $\begin{array}{l}\text { Total } \\
1988\end{array}$ & $\begin{array}{c}\text { December } \\
1988\end{array}$ & $\begin{array}{c}\text { Novernber } \\
1988\end{array}$ & $\begin{array}{c}\text { October } \\
1988\end{array}$ \\
\hline 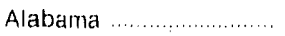 & 2.08 & 2.21 & 2.56 & 2.34 & 2.15 & 2.14 & 2.02 & 2.07 \\
\hline 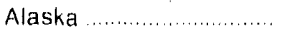 & -. & -. & - & $\cdots$ & 1.34 & $\cdots$ & $\ldots$ & $\ldots$ \\
\hline 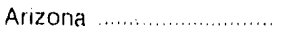 & 215 & 2.09 & 2.19 & 2.84 & 2.32 & 2.50 & 2.44 & 2.42 \\
\hline 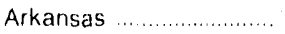 & 1.50 & $1.59^{\circ}$ & 1.47 & 1.21 & 1.39 & 1.10 & 1.30 & 1.47 \\
\hline Calıfornia ........................ & 2.75 & 2.89 & 2.92 & 3.33 & 2.93 & 3.65 & 3.66 & $3.18^{\prime}$ \\
\hline Colorado ............................. & 2.18 & 2.14 & 2.10 & 2.07 & 2.25 & 2.08 & 2.77 & 2.38 \\
\hline Connecticut ...................... & 2.81 & 2.87 & 3.13 & 3.19 & 2.2 .4 & 3.43 & 2.72 & 2.72 \\
\hline 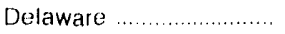 & 2.61 & 2.56 & 2.72 & 3.28 & 2.59 & 3.09 & 2.76 & 2.69 \\
\hline District of Columbia ....... & -- & -- & -.- & -- & - & -- & $\cdots-$ & $\cdots$ \\
\hline Florida ............................. & 2.63 & 2.28 & 2.34 & 2.37 & 2.12 & 2.26 & 2.18 & 1.70 \\
\hline 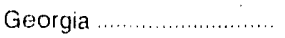 & 2.76 & 3.60 & 3.62 & 3.35 & 2.80 & 3.50 & 3.16 & 2.78 \\
\hline 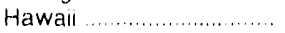 & -. &.- & $\ldots$ & -. & -. & $\ldots$ & -- & $\ldots$ \\
\hline 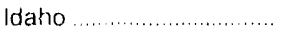 & -.. & $\ldots$ & ... & -.. & - & $\ldots$ & .. & $\ldots$ \\
\hline 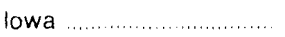 & 2.61 & 2.84 & 3.19 & 3.49 & 2.04 & 3.26 & 3.03 & 2.72 \\
\hline 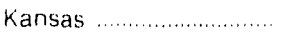 & 2.27 & 2.07 & 2.27 & 2.37 & 2.05 & 2.09 & 2.10 & 1.90 \\
\hline 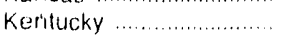 & 2.98 & 2.76 & 2.81 & 3.07 & 2.52 & 3.25 & 2.83 & 2.53 \\
\hline 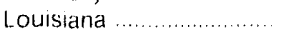 & 1.66 & 1.54 & 1.81 & 1.80 & 1.70 & 1.63 & 1.82 & 1.73 \\
\hline 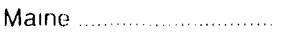 & $\ldots$ & -. & $-\cdots$ & $\cdots$ & $\cdots$ & ... & -.. & $\cdots$ \\
\hline 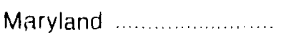 & 2.60 & 2.64 & 3.67 & 3.41 & 2.71 & 3.86 & 3.29 & 2.52 \\
\hline Massachuselts ............. & 2.39 & 2.55 & 3.17 & 5.22 & 2.29 & 4.31 & 2.67 & 2.84 \\
\hline Michigan ........................... & .18 & .20 & .19 & 20 & .41 & .28 & 1.13 & .44 \\
\hline 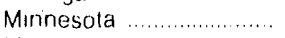 & 1.78 & 2.08 & 2.53 & 2.87 & 1.99 & 2.59 & 2.07 & 2.25 \\
\hline Mississipp $\neq \ldots \ldots \ldots \ldots \ldots \ldots$ & 1.68 & 1.67 & 1.96 & 2.23 & 1.90 & 2.28 & 1.97 & 1.93 \\
\hline 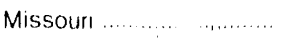 & 2.36 & 2.43 & 2.60 & 2.56 & 2.75 & 2.71 & 2.38 & 1.82 \\
\hline 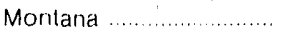 & 1.38 & 1.12 & 1.01 & 1.18 & 1.53 & 1.29 & 1.72 & 1.64 \\
\hline Nebraska ......................... & 2.28 & 2.36 & 2.50 & 2.89 & 2.56 & 2.99 & 2.43 & 2.27 \\
\hline Nevada & 1.72 & 2.09 & 2.00 & 2.15 & 2.79 & 2.07 & 2.61 & 2.88 \\
\hline New Harnpshure . ............ & $\cdots$ & -- & -. & $\cdots$ & 2.48 & $\cdots$ & $\cdots$ & $-\cdot$ \\
\hline New York ......................... & 2.37 & 2.51 & 2.78 & 2.98 & 2.30 & 2.99 & 2.56 & 2.35 \\
\hline North Carolina ............... & - & -. & -. & $\ldots$ & 3.48 & $\ldots$ & $\ldots$ & ... \\
\hline North Dakota .................. & 4.20 & -. & $\cdots$ & $\cdots$ & 4.56 & .. & 1.42 & 4.37 \\
\hline 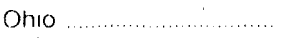 & 3.70 & 4.25 & 123 & 4.21 & 3.51 & 4.18 & 4.17 & 3.83 \\
\hline 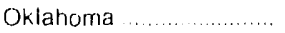 & 296 & 2.98 & 3.13 & 3.03 & 2.92 & 2.54 & 3.15 & 3.01 \\
\hline 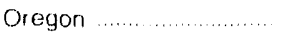 & 1 &.- & ... & $\ldots$ & -..- & ..... & -.. & $\ldots$ \\
\hline Pennsylvaria ....................... & 3.78 & 3.66 & 3.97 & 3.81 & 3.45 & 4.03 & 3.78 & 3.62 \\
\hline Rhode Island ................ & 273 & $\cdots$ & -- & $-\cdots \cdot$ & 215 & $\cdots$ & $\cdots$ & $\cdots$ \\
\hline South Carolina ................... & 2.31 & 2.26 & 4.30 & 3.94 & 1.86 & 3.90 & 3.33 & 3.49 \\
\hline South Dakota ................ & - & $\ldots-$ & $\ldots$ & $\ldots$ & 2.33 & ..... & ...... &.- \\
\hline Tennessee & $\ldots$ & $\ldots$ & .. & $\ldots$ & 2.46 & & $\ldots$ & $\ldots$ \\
\hline 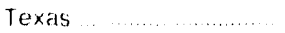 & 2.13 & 2.14 & 225 & 2.48 & 2.16 & 2.39 & 2.32 & 2.13 \\
\hline 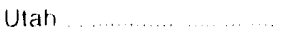 & $\cdots$ & $\cdots$ & $\cdots$ & $\ldots$ & 3.05 & $\cdots$ & $\ldots$ & ... \\
\hline Vermont & $\ldots$ & $\ldots$ & $\ldots$ & $\ldots$ & $\ldots$ & $\ldots$. & .. & $\ldots .$. \\
\hline 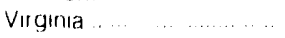 & 2.74 & 133 & 2.34 & 2.04 & 2.16 & 5.06 & 1,34 & 4.00 \\
\hline Washngton & $\ldots$ & $\ldots$ & $\ldots$ & 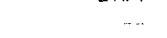 & 315 & $\ldots$ & ... & $\ldots$ \\
\hline West Virginta & 4.76 & 4.45 & 4.31 & 476 & 3.89 & 4.76 & 4.52 & 434 \\
\hline 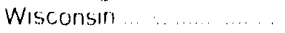 & 3.17 & 3.43 & $35 \%$ & 3.43 & 326 & $3.6 \%$ & 3.78 & 3.31 \\
\hline 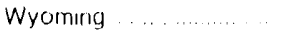 & 3.45 & 5.17 & 4.10 & 357 & 3.78 & 360 & 4.03 & 415 \\
\hline Total & 231 & 2.33 & 244 & 264 & 233 & 257 & 2.58 & 2.40 \\
\hline
\end{tabular}

\footnotetext{
a Includes all steam electric utility generatirg plants with a combined capacity of 50 megawalts or greater

- Avefage prices calculated from data reported on Form ElA-176 Not Appticatio

Notes and Sources: See the last page of thus sectort.
} 
Table 32. Average Price of Natural Gas Deilvered to All Consumers by State (Dollars per Thousand Cubic Feet)

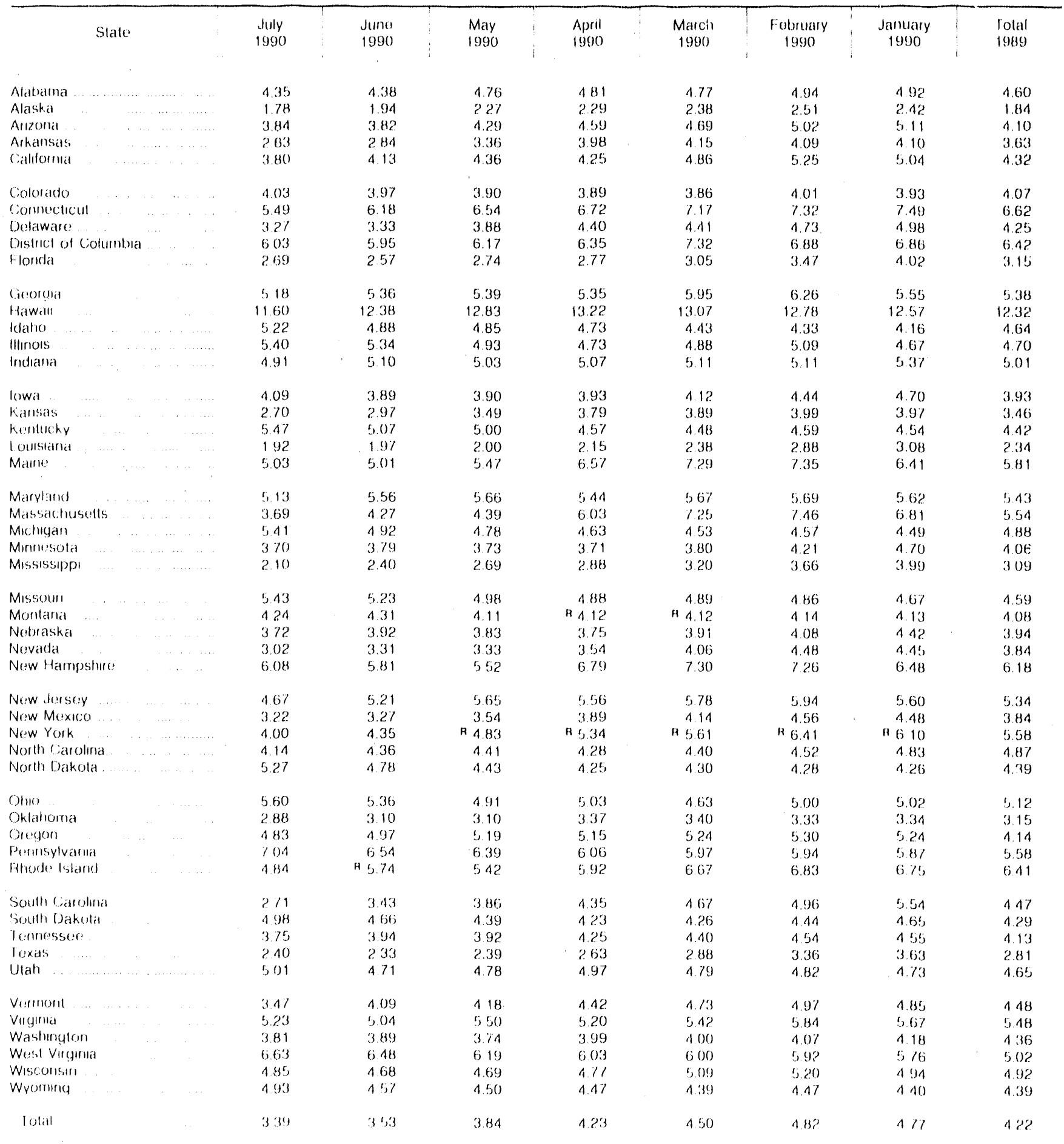

Soe tomolnotes at und of table: 
Table 32. Average Price of Natural Gas Delivered to All Consumers by State (Continued)

(Dollars per Thousand Cubic Feet)

\begin{tabular}{|c|c|c|c|c|c|c|c|c|}
\hline Slate & $\begin{array}{c}\text { Decomber } \\
1989\end{array}$ & $\begin{array}{c}\text { Novombor } \\
1989\end{array}$ & $\begin{array}{l}\text { Oclobon } \\
1989\end{array}$ & $\begin{array}{c}\text { Soptombor } \\
1989\end{array}$ & $\begin{array}{c}\text { August } \\
1089\end{array}$ & $\begin{array}{l}\text { July } \\
\text { lese }\end{array}$ & $\begin{array}{l}\text { Jurie } \\
\text { lges }\end{array}$ & $\begin{array}{l}\text { May } \\
1989\end{array}$ \\
\hline$\ldots \ldots \ldots \ldots$ & 4.91 & 4.78 & 456 & d.5is & 4.51 & 4.52 & 435 & 4.41 \\
\hline Alaskáa & 2.20 & $2.16 i$ & 198 & 1.77 & 1.63 & 1.70 & $18 \%$ & 1.91 \\
\hline Alzona $\ldots \ldots$ & 4.64 & 368 & 3.45 & 3.49 & 3.18 & 3.31 & 3.70 & 3.84 \\
\hline Arkallsas & 3.86 & $36 ?$ & $35 \%$ & 3.66 & 3.09 & 324 & 3.45 & 3.85 \\
\hline Cathorma .... & 4.87 & 4.37 & 3.74 & $39 ?$ & 392 & 370 & 4.00 & 4.28 \\
\hline Colorado $\quad \ldots \ldots \ldots \ldots \ldots . . . . .$. & 411 & 4.22 & 423 & 4.29 & $30 ?$ & 4.31 & 4.36 & 4.17 \\
\hline 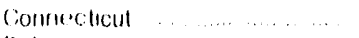 & 7.25 & 6.61 & 5.93 & 5.73 & 5.93 & 6,51 & 6.26 & 6.05 \\
\hline Dolawaro $\quad \ldots$ & $4.9 ?$ & 4.33 & 3.69 & 3.46 & $3.5 ?$ & 3.50 & 4.29 & $4.2 \%$ \\
\hline Districi of ciolumbia & (i.57 & 640 & 642 & 652 & 5.10 & 5.86 & 5.21 & 6.05 \\
\hline Flonida $\ldots \quad \ldots \quad \ldots \quad \ldots$ & 383 & 3.18 & 3.04 & 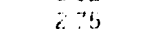 & 2.83 & 3.04 & 3.05 & 3.26 \\
\hline Goorgla $\quad \ldots \quad \ldots . . . . . . .$. & 5.30 & 560 & 5.70 & 5.47 & 5.44 & 5.66 & 5.39 & 5.46 \\
\hline 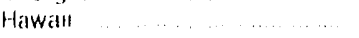 & 1642 & 1271 & 12.40 & 12.53 & 1264 & 12.81 & 1296 & 12.34 \\
\hline tdaho $\quad \ldots \ldots+\ldots \ldots$ & 445 & 4.89 & 5.04 & 5.11 & 5.33 & 5.10 & 505 & $5.0 ?$ \\
\hline Illinols $\quad \ldots . . . .$. & 443 & 4.37 & 460 & 512 & 5.42 & 5.14 & 500 & 4.79 \\
\hline Indiana $\ldots . . . \quad \ldots \ldots \ldots$ & 4.59 & 4.52 & 4.59 & 503 & 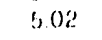 & 4.85 & 4.69 & 5.47 \\
\hline$\ldots$ & 4.26 & 3.88 & 3.56 & 369 & 3.85 & .3 .78 & 3.72 & 366 \\
\hline Kansas . . . . . . . & 3.92 & 3.71 & 3.81 & 3.27 & 280 & 2.90 & 3.15 & 3.59 \\
\hline Kentucky . . . . . . . . & 423 & 4.16 & 4.60 & 5.04 & 5.05 & 4.78 & 4.64 & 4.56 \\
\hline 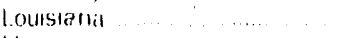 & 2.90 & 233 & 2.10 & 2.10 & 2.11 & 2.14 & 2.14 & 2.09 \\
\hline Manre $\ldots \ldots \ldots$ & 6.37 & c. 96 & 5.56 & 5.42 & 5.21 & 5.10 & 5.37 & 5.50 \\
\hline Maryland & 5.55 & 5.81 & 5.04 & 5.66 & 509 & 5.48 & 5,06 & 5.14 \\
\hline Massachusetts _............. & 6.77 & 6.22 & 3.93 & 4.04 & 4.13 & 4.10 & 4.41 & 4.83 \\
\hline 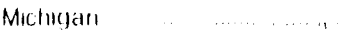 & 4.54 & 471 & 5.17 & 5.62 & 600 & 5.53 & 5.33 & 4.94 \\
\hline Munnosola & 427 & 3.95 & 3.82 & 3.92 & 3.84 & 3.80 & 3.91 & 3.86 \\
\hline Mississippl & 366 & 328 & 267 & 2.52 & 2.44 & 2.19 & 2.62 & 2.65 \\
\hline Misisoun & 456 & 4.70 & 4.89 & 5.19 & 5.32 & 5.34 & 5.2 .1 & 4.85 \\
\hline$\ldots$ & 417 & 419 & 3.88 & 3.81 & 3.68 & 3.44 & 3.99 & 3.94 \\
\hline 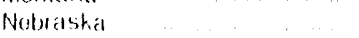 & $41 \%$ & 3.87 & 3.77 & 3.85 & 374 & 3.66 & 4.07 & 3.89 \\
\hline Nevata & 439 & 4.04 & 360 & 3.27 & 3.06 & 3.07 & 3.70 & 0.73 \\
\hline Now Hannoshure: & $6 ! 7$ & 667 & 55.3 & 5.17 & 582 & 5.75 & 5.49 & 5.47 \\
\hline New Jersey $\ldots \ldots$ & 5.81 & 5.63 & 5.30 & 4.94 & 465 & 4.54 & 4.54 & 5.19 \\
\hline New Mexico & 4.12 & 381 & 3.62 & 3.43 & 3.47 & 3.61 & 3.69 & 3.64 \\
\hline New rouk . . . . . . & 619 & 5.94 & 5.00 & 4.63 & 4.59 & 4.60 & 4.81 & 499 \\
\hline North Carolina & b! & 4.57 & 4.69 & 465 & 4.61 & 4.55 & 4.56 & 4.67 \\
\hline Norlth Dakolat & 425 & 4.33 & 4.78 & 477 & 5.33 & 5.09 & 4.80 & 1.61 \\
\hline Ohne ... & $A B 1$ & 5.03 & 5,03 & 5.56 & 5.73 & 5.68 & 5.53 & 5.04 \\
\hline$\ldots, \quad \ldots$ & 3.33 & 318 & 329 & 296 & 2. By & 3.10 & 3.083 & 3.07 \\
\hline Oreyon & $5.2 B$ & 510 & 187 & 496 & 5.04 & 5.12 & 5.04 & 505 \\
\hline Pontisylvantá & 4,65 & 5.80 & 5.99 & 623 & 6.20 & 6.26 & 5.62 & 5.55 \\
\hline Rhorde: Isiand & $6 \theta 8\}$ & 645 & 545 & 614 & 5.53 & 5.71 & 6.15 & 6.25 \\
\hline Soulh Canolina & 56 & 437 & 3.837 & 371 & 3.64 & 358 & 3.62 & 4.22 \\
\hline Soulh loakola & 443 & 4.14 & 414 & 447 & 463 & 457 & 441 & 1.28 \\
\hline Tonterseser & 445 & 4.17 & 393 & 393 & 387 & 3.93 & 3.92 & 3.883 \\
\hline$\ldots$ & $3: 7$ & 286 & 2.54 & 2. 4? & 255 & 2.55 & 2.56 & 2.55 \\
\hline Wah & 477 & $49 \%$ & 508 & 487 & 5.06 & 4.68 & 4.46 & 4.60 \\
\hline Verrmont & 493 & 439 & 413 & 4.26 & 137 & 4.47 & 4.47 & 154 \\
\hline Vircyrnes & 679 & 533 & 513 & $5: 7$ & 4.82 & 529 & 1.66 & 5.11 \\
\hline Watshomplon & 130 & 4.38 & 3.14 & 407 & 3.86 & 394 & 1.16 & 4.23 \\
\hline Wensl Virginla & 5,6, & 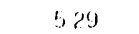 & 489 & $4 \mathrm{HO}$ & 4.98 & 503 & 5.11 & 4.90 \\
\hline Whecolesent & $46 f 5$ & 4.87 & 4.77 & $5(0)$ & 589 & 516 & 4.89 & 4.85 \\
\hline Wyomming & 434 & 445 & $A 4 B$ & 450 & $4 / 9$ & 471 & 4.46 & 4.36 \\
\hline$|0| a \mid$ & 161 & 430 & $3.86 \%$ & $3.6 \%$ & 348 & 369 & $3.7 \%$ & 3.94 \\
\hline
\end{tabular}

Ser foothotel at ond ol table 
Table 32. Average Price of Natural Gas Delivered to All Consumers by State (Continued)

(Dollars per Thousand Cubic Feet)

\begin{tabular}{|c|c|c|c|c|c|}
\hline Slathe & $\begin{array}{l}\text { Almul } \\
\text { I!Is!3 }\end{array}$ & $\begin{array}{c}\text { Malich } \\
\text { ind }\end{array}$ & $\begin{array}{c}\text { lobmualy } \\
\text { lesses }\end{array}$ & $\begin{array}{c}\text { Jarmaty } \\
19180\end{array}$ & 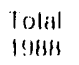 \\
\hline Alalsambal & 446 & $4 d 1$ & d lifi & 473 & $1 \leq 1,1$ \\
\hline Alalsitial & $? 11$ & $21 \%$ & $a d i$ & 223 & 1.17 \\
\hline Allzonad & 361 & $4 t, 7$ & 50 & 5.17 & 176 \\
\hline Arkallsals & 35,3 & 35,1 & 379 & 'j 98 & 367 \\
\hline Cialiformal & 398 & 443 & A $1:$ & 511 & 1: \\
\hline colonardo & 40,3 & 401 & $: 196$ & 390 & 404 \\
\hline Comenectent & 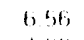 & 619 & 69 & 6869 & 6.25 \\
\hline 1)elawars & $40 \mathrm{H}$ & $4 ! 1$ & 478 & $; 10$ & 1.2? \\
\hline 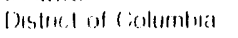 & $63 \%$ & 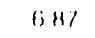 & $6 ! 66$ & 6,67 & $6.0 \%$ \\
\hline I lonila & 326 & 311 & $3: 6$ & 336 & $\therefore 9 ?$ \\
\hline Sincongla & 4. & $3: 39$ & 1,383 & 516 & 5.24 \\
\hline Hawilt & 1242 & 1198 & $114{ }^{\prime}$ & $11: 34$ & $\left|a^{\prime}\right| \mid$ \\
\hline letation & 400 & 453 & 439 & 4.16 & 499 \\
\hline HHorols , & 478 & 403 & $A \mathrm{BC}^{2}$ & 454 & 4.36 \\
\hline Indialla & 540 & $\therefore: 10$ & 5.00 & 540 & 473 \\
\hline lowa & $\therefore 68\}$ & 319 & $40 t$ & $4 a$ & 400 \\
\hline kallsals & $34 c^{\prime}$ & 38,3 & 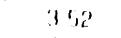 & 341 & 319 \\
\hline kinoluck.y & 443 & 933 & $43 !$ & 431 & 120 \\
\hline 1 oulstatas & 290 & 242 & 979 & $38 \%$ & 229 \\
\hline Mallut: & 519 & $5(18)$ & 5,47 & 0.01 & 580 \\
\hline Maryland. & 306 & 546 & $5 / 4$ & 562 & 531 \\
\hline Massacthusells & 5,3 & 619 & 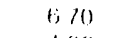 & (i.fij & $56 ?$ \\
\hline Michuyan & $48(3)$ & $A B{ }^{\circ}$ & $A B r$ & 4.834 & $50 \pi$ \\
\hline Mirubesola & 384 & $3 ! 14$ & 116 & 4.34 & Aot \\
\hline Miscissif)| & 307 & 340 & 393 & 4.00 & 340 \\
\hline Missoun & $4 \vdots 1$ & 431 & 438 & 436 & 444 \\
\hline Montanta & 4014 & 413 & 117 & 1.25 & 401 \\
\hline Noterasha & $1 / 5$ & $: 184$ & 406 & $1: 1$ & 3.88 \\
\hline fonada & 350 & $A 19$ & 143 & 4.41 & $4 \leq \%$ \\
\hline New Hanpsshers. & 616 & 641 & ball & 6.34 & 66.4 \\
\hline Now bersity & 3.24 & 539 & 570 & $56 ?$ & 5,18 \\
\hline New Mrexico & $1 \%$ & $3 \mathrm{HO}_{2}$ & $40 \%$ & 419 & $3.6 \mathrm{H}$ \\
\hline New rork & 534 & 6.21 & 614 & 6.16 & 526 \\
\hline North Carrolina & 490 & $50 \mathrm{~s}$ & 501 & 503 & $4.6 \%$ \\
\hline Forth Dakrota & 43 & 426 & 420 & 430 & 470 \\
\hline Oho & 59 & 507 & 5.21 & 519 & $4 ! 98$ \\
\hline Oklahoma & 311 & 391 & $3 \% 6$ & 316 & 315 \\
\hline Orregen & 1,083 & \$10 & 520 & 549 & t) \\
\hline Pencrisyluarlial & 3 & $5: 38$ & 534 & $5: 1 \%$ & 5.18 \\
\hline Hhorde Is,anil & $\Leftrightarrow 5$ & 677 & 6.89 & 648 & $6 ; 14$ \\
\hline Sombli Carsolusa & 1135 & 496 & $5: 3 H$ & 5.37 & 449 \\
\hline Sombl Dakota & 414 & 414 & $4 \%$ & 446 & 434 \\
\hline Termessst;e: & $A \cup A$ & 110 & $4 \%$ & A.ts & 307 \\
\hline Toxas & $2 / 10$ & ?93 & 311 & $: 13 \%$ & $? 69$ \\
\hline Utah & 473 & 4 & $44 !$ & 452 & 4.47 \\
\hline Vormonel & $A d B$ & A.dB & 4 & 4.49 & 1.14 \\
\hline Vurguna & $5(1) 9$ & $56 ?$ & $5 / 1$ & $5,(60$ & $48 \%$ \\
\hline Washluegenton & 436 & $44 \%$ & 450 & 443 & 419 \\
\hline Wostil Virginata & $a s(1)$ & $4 ! 3)$ & 411 & 476 & ij 11 \\
\hline Wiscortsill & $49 \%$ & 196 & $\sin 8$ & $50 ?$ & 9.19 \\
\hline Wyomming & $4: 38$ & $43 \%$ & $4: 1 \%$ & $43 \%$ & 418 \\
\hline lolal & 418 & 446 & 460 & 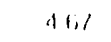 & $40 ! 1$ \\
\hline
\end{tabular}

H Rreviened hata

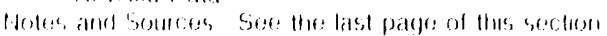




\section{Table 33. Percentage of Total Deliveries Represented by Onsystem Sales}

\begin{tabular}{|c|c|c|c|c|c|c|c|c|}
\hline \multirow{2}{*}{ Slate } & \multicolumn{2}{|c|}{$\begin{array}{c}\text { Auguss } \\
1900\end{array}$} & \multicolumn{2}{|c|}{$\begin{array}{l}\text { July } \\
1090\end{array}$} & \multicolumn{2}{|c|}{$\begin{array}{l}\text { Ju1no } \\
1900\end{array}$} & \multicolumn{2}{|c|}{$\begin{array}{l}\text { Maly } \\
1090\end{array}$} \\
\hline & Commorial & Indusstral & Commoncial & Industraal & Cormmorcial & Induslelal & Commorckal & Indusilial \\
\hline Alabarnal & 71.3 & 361 & 74.8 & $29 ! 9$ & 78.3 & 32.7 & 72.7 & 2) 2 \\
\hline Alaska $\ldots \ldots \ldots \ldots$ & 100.0 & 96.3 & 100.0 & 57.4 & 100.0 & 50.1 & 100.0 & 640 \\
\hline Allogollit $\ldots \ldots \ldots \ldots$ & 906 & 25.6 & $01 \%$ & 220 & 925 & 29.6 & 84.7 & 45.1 \\
\hline Arkallsals $\ldots . . . . . . . . . . . . . . . . . .$. & 86.8 & 193 & 88.4 & 18.3 & H.1 7 & 16.8 & 90.6 & 16.7 \\
\hline Califorma & 82.4 & 3810 & 477.0 & $A .30 .9$ & 1136.6 & $" 130.4$ & H 90.1 & $n+1.8$ \\
\hline Colorado ... & 94.4 & 39.7 & 04,6 & 56.0 & 97.4 & 394 & 07.2 & 196 \\
\hline Cionfoclicul & 86.8 & 65.9 & 87.4 & 67.2 & 80.9 & 78.6 & 01.1 & 700 \\
\hline Dolawalro ........ & 100.0 & 73.7 & 100.0 & 69.9 & 100,0 & 70.6 & 100.0 & 67.5 \\
\hline Disilice of Columbia ................. & 100.0 & $\cdots$ & 100.0 & $\cdots$ & 100.0 & $\ldots$ & 100.0 & $\rightarrow \cdot$ \\
\hline Flonda $\ldots . . . . . . . . . . . . . . . . . .$. & 97.2 & 38.9 & 97.4 & 59.7 & 97.2 & 61.0 & 97.3 & 62.5 \\
\hline 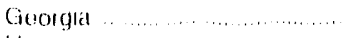 & 81.6 & 40.1 & 79.0 & 38.9 & 79.9 & 31.7 & 81.0 & 276 \\
\hline Hawall & 1000 & - & 100.0 & $\cdots$ & 100.0 & $\cdots$ & 1000 & -- \\
\hline Idaho $\ldots \ldots \ldots \ldots \ldots \ldots \ldots$ & 85.2 & 3 & 86.2 & 5 & 88.1 & 6 & 85.8 & 6 \\
\hline Illinots $\ldots \ldots \ldots \ldots$ & 460 & 9.4 & 16.8 & H 13.7 & 47.4 & B.6 & 50.5 & 14.9 \\
\hline Inclianal $\ldots \ldots \ldots \ldots \ldots \ldots \ldots \ldots$ & 89.8 & 15.6 & 92.2 & 16.7 & 90.7 & 16.0 & $9 \% 1$ & 159 \\
\hline lowal $\ldots \ldots \ldots \ldots \ldots \ldots \ldots \ldots$ & 95.6 & $3 a 5$ & 953 & 31.8 & 06.3 & 30.5 & 97.2 & 29.6 \\
\hline 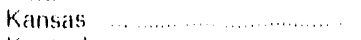 & 94.3 & 17.3 & 94.5 & 19.5 & 93.6 & 16.3 & 92.2 & 16.0 \\
\hline 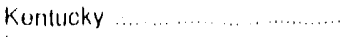 & 89.2 & 10.8 & 89.3 & 11.5 & 89.6 & 12.5 & 89.9 & 11.5 \\
\hline 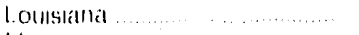 & 100.0 & 448 & 100.0 & 49.7 & 100.0 & 14.3 & 100.0 & 44.7 \\
\hline 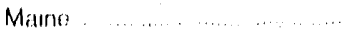 & 1000 & 1000 & 100.0 & 100.0 & 100.0 & 100.0 & 100.0 & 100.0 \\
\hline 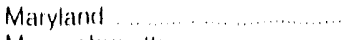 & 93.3 & 190 & 93.8 & 19.2 & 94.7 & 20.3 & 94.5 & 27.6 \\
\hline Massachusells .......................... & 99.8 & 95.9 & 100.0 & 97.4 & 100.0 & 92.7 & 100.3 & 946 \\
\hline Michigan ............................... & 49.5 & 8.3 & 17.7 & 0.4 & 56.2 & 9.9 & 65.6 & 15.2 \\
\hline Minnosola ................................. & 89.4 & 28.8 & 89.6 & 2.7 .0 & 91.0 & 32.0 & 94.7 & 30.5 \\
\hline 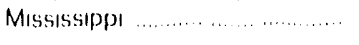 & 94.0 & 44.4 & 89.8 & 42.5 & 00.4 & 14.1 & 90.9 & 449 \\
\hline Missoull. & 724 & 22.0 & 72.5 & 22.3 & 78.8 & 23.0 & 83.7 & 27.7 \\
\hline Montana $\ldots . . . \ldots \ldots \ldots . . . . . . . . . . . .$. & 96.9 & 75.5 & 96.3 & $77.0^{\circ}$ & 98.4 & 79.7 & 96.9 & 82.1 \\
\hline 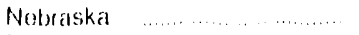 & 94.1 & 30.5 & 96.8 & 1128.2 & 89.3 & F 31.1 & 92.1 & 34.4 \\
\hline 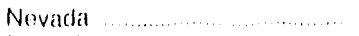 & 96.7 & 8.6 & 90.5 & 8.6 & 96.7 & 9.9 & 96.4 & 10.6 \\
\hline Now Hampshure $\ldots . . . . . . . . . . . .$. & 100.0 & 100.0 & 100.0 & 100.0 & 100.0 & 100.0 & 100.0 & 100.0 \\
\hline Now Jursey ....................... & 86.4 & 459 & $89 . \%$ & 48.8 & 93.2 & 50.4 & 94.3 & 51.6 \\
\hline 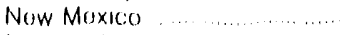 & 73.0 & 21.3 & 76.3 & 34.1 & $7 C 7$ & 28.0 & 78.2 & 18.1 \\
\hline 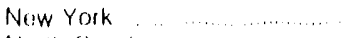 & 83.4 & 29.5 & $H 2.1$ & 31.9 & 81.9 & 280 & ค 82.4 & 33.2 \\
\hline 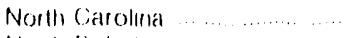 & 833.1 & 45.8 & 83.4 & 53.8 & 84.8 & 48.3 & 89.2 & 47.7 \\
\hline 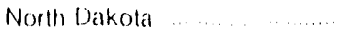 & 53.4 & 17.1 & 54.3 & $19 . ?$ & 69.7 & 20.3 & $7 \leqslant .4$ & 22.2 \\
\hline.$\quad \ldots \ldots$ & 749 & 96 & 76.4 & 8.9 & 77.9 & 9.4 & 81.8 & 9.3 \\
\hline Oklahoma $\therefore . . . . . . . . .$. & 885 & 45.8 & 88.1 & 49.8 & 89.8 & 363.9 & 91.9 & 13.6 \\
\hline Orogon $\ldots \ldots \ldots \ldots \ldots$ & 965 & 15.3 & 965 & 16.4 & 97.5 & 23.4 & 97.1 & 18.2 \\
\hline Punmisylvarial ...... . . . . . & 671 & 15.6 & 660 & 14.0 & 69.7 & 167 & 73.6 & 15.3 \\
\hline Fhode Island . . . ................. & 100,0 & 814 & 1000 & 82.2 & 100.0 & $n 85.4$ & 100.0 & 88.6 \\
\hline South (Carolma $\quad \ldots . . . . .$. & 970 & 79.1 & 97.1 & 81.2 & 97.1 & 77.6 & 90.6 & 74.3 \\
\hline South Oakola & 849 & 325 & 855 & 38.5 & $86 . ?$ & 42.4 & 87.1 & 55.4 \\
\hline Ionnosstes $\quad \ldots . \quad \ldots$ & 951 & 51.4 & 94.2 & 53.0 & 94.5 & 52.1 & 94.6 & 52.7 \\
\hline Toxas $\quad \ldots \ldots \ldots \ldots$ & 944 & 24.7 & 94.1 & 24.8 & 95.4 & 236 & 94.1 & 21.0 \\
\hline Mah . . . . . . . & 100.0 & 197 & 100.0 & 19.1 & 1000 & 18.7 & 100.0 & 18.5 \\
\hline Venmonil & 1000 & 100.0 & 1000 & 100.0 & 1000 & 100.0 & 100.0 & 1000 \\
\hline Virgunia & 914 & 13.4 & 938 & 10.0 & 936 & 12.9 & 938 & 16.0 \\
\hline Wastimenton & 8385 & 177 & 881 & 46.2 & 88.9 & 47.7 & 01.0 & 50.1 \\
\hline Wesil Virgirha & 369 & 109 & 36.4 & 11.1 & 37.2 & $10\}$ & 49.2 & 10.8 \\
\hline Wisconsir! $\ldots . .$. & 80.1 & $30 . ?$ & $79) 7$ & 306 & 83.5 & 29.8 & 88.9 & 354 \\
\hline Wyominn! $\ldots \ldots \ldots$ & 99.6 & 6.? & 996 & $6 . ?$ & 99.7 & 77 & 99.7 & 11.8 \\
\hline$\ldots \ldots \ldots \ldots$ & 833.4 & 31.8 & 183.4 & A $: 116$ & 185.3 & 14296 & 1186.4 & H 30.2 \\
\hline
\end{tabular}

Soo loothotes, al end of tables 
Table 33. Percentage of Total Deliveries Represented by Onsystem Sales (Continued)

\begin{tabular}{|c|c|c|c|c|c|c|c|c|}
\hline \multirow[t]{2}{*}{ Stale } & \multicolumn{2}{|c|}{$\begin{array}{l}\text { Aprol } \\
\mid(t), 0\end{array}$} & \multicolumn{2}{|c|}{$\begin{array}{c}\text { Match } \\
1000\end{array}$} & \multicolumn{2}{|c|}{$\begin{array}{c}\text { Fobruary } \\
1990\end{array}$} & \multicolumn{2}{|c|}{$\begin{array}{c}\text { January } \\
\text { Iggo }\end{array}$} \\
\hline & Commercial & Industual & Commorcial & Indusitrial & Commorcial & Inclusleial & Commorcial & Inclustrial \\
\hline 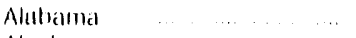 & 74.8 & 27.4 & 78.1 & 2019 & 80.4 & 2819 & 86.5 & 31.8 \\
\hline$\cdots \quad \cdots \quad \cdots, \cdots$ & 100,0 & 561 & 100.0 & 00.5 & 100.0 & 60.3 & 100.0 & 67.8 \\
\hline 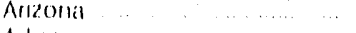 & 903 & 12.7 & $9 / 4.9$ & 47.2 & 96.5 & $5 B Q$ & 06.4 & 62.3 \\
\hline Arkallsalss $\ldots \ldots . . . . . .$. & 932 & 17.6 & 03.7 & 14.5 & 047 & 16.7 & 96.8 & 18.1 \\
\hline 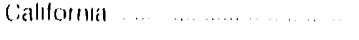 & 1190.3 & 1140.6 & 104.4 & $H 46.8$ & A 81.0 & $11: 37.0$ & $n 95.8$ & 1157.5 \\
\hline$\ldots \ldots, \quad \ldots \ldots \ldots$ & 07.6 & 46.7 & 97.81 & 45.6 & 98.0 & 46.6 & 08.1 & 50.6 \\
\hline$\ldots \ldots \ldots \ldots$ & $9 ! 6$ & 86.2 & 93.6 & 93.9 & 100.0 & 018.5 & 100.0 & 09.7 \\
\hline 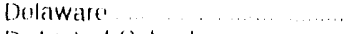 & 1000 & 69.9 & 100.0 & 74.0 & 100,0 & 72.2 & 100.0 & 78.2 \\
\hline Disletct of Collumblat $\ldots \ldots$ & 1000 & $\cdots$ & 100.0 & $\cdots$ & 100.0 & $\cdots$ & 100.0 & $\cdots$ \\
\hline Flonta , $\quad \cdots \cdots$ & 1000 & $74 . ?$ & 100.0 & 72.1 & 100.0 & 72.5 & 100.0 & 724 \\
\hline Goorgla $\ldots . .$. & $86 . ?$ & 27.4 & 87.9 & 27.2 & 89.8 & 25.3 & 96.3 & $5\} 3 . ?$ \\
\hline Hawall $\ldots . .$. & 100.0 & $\cdots$ & 100.0 & $\cdots$ & 100.0 & $\cdots$ & 100.0 & $\cdots$ \\
\hline Idlaho $\ldots . . . \ldots \ldots \ldots$ & 817.7 & 6 & 80.7 & 6 & 90.4 & .6 & 91.5 & 8.8 \\
\hline 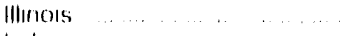 & 57.9 & 16.8 & 62.5 & 22.3 & 67.6 & 31.4 & (ifi.ti & $20 !$ \\
\hline$\ldots \ldots \ldots$ & $y(1.2$ & 18.5 & 0335 & 18.4 & 98,6 & 283.3 & 09.2 & 20.3 \\
\hline$\ldots \ldots \ldots \ldots$ & 07.8 & 34.2 & ()$(3.1$ & 390 & 98.2 & 46.2 & 98.6 & 44.5 \\
\hline 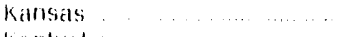 & 930 & 18.4 & 01.0 & 17.5 & 011 & 19.6 & 929 & 21.8 \\
\hline kenlucky $\quad \ldots \ldots \ldots \ldots . . . .$. & 93.4 & 13.0 & 95.3 & 15.5 & (1)6.? & 218 & 96.3 & 10.1 \\
\hline 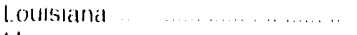 & 100.0 & 45.3 & 100.0 & 410 & 100.0 & 41.9 & 100.0 & 41.1 \\
\hline Manmo $\quad \ldots \ldots \ldots \ldots \ldots$ & 100.0 & 100.0 & 100.0 & 1000 & 100.0 & 100.0 & 100.0 & 1000 \\
\hline 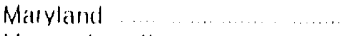 & 958 & 36.9 & 96.1 & 42.1 & 97.2 & 699 & 98.3 & 58.7 \\
\hline 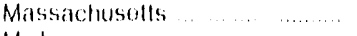 & 100.0 & 9014 & 100.0 & 90.2. & 100.0 & 918 & 100.0 & $94 . ?$ \\
\hline Michonan $\quad . . . . . . . . . . . . .$. & 70.7 & 189 & 730 & 22.0 & 72.4 & 207 & 715 & 22.0 \\
\hline 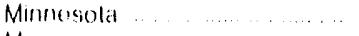 & 94.7 & 35.3 & $96 ; 6$ & dG0 & 96.2 & 51.4 & 97.6 & 53.5 \\
\hline Mıssisssipp| $\quad \ldots \ldots \ldots \ldots \ldots$ & 100.0 & 54.3 & 100.0 & 57.0 & 100.0 & 51.4 & 100.0 & 520 \\
\hline MissouII $\ldots \ldots \ldots \ldots$ & 890 & 327 & 80.9 & 37.7 & 91.4 & 46.3 & 94.1 & 56.8 \\
\hline Montanal $\ldots \ldots$ & 986 & 750 & 97.7 & $6\} 3.8$ & 97.8 & 733 & $9 \pi 9$ & 56.5 \\
\hline Nobraskat $\ldots \ldots$ & 92.7 & 39.9 & 93.9 & 43.8 & 95.2 & 56.5 & 965 & 59.6 \\
\hline 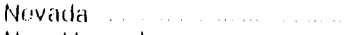 & 970 & 11.3 & 97.7 & 11.8 & 99.4 & 18.8 & 99.1 & 27.8 \\
\hline New Hampshire & 100.0 & 100.0 & 100.0 & 100.0 & 100.0 & 100.0 & 1000 & 100.0 \\
\hline$\ldots \ldots \ldots \ldots \ldots$ & 64.4 & 59.8 & 96.6 & 01.4 & 09.3 & 73.2 & 99.6 & 81.3 \\
\hline$\ldots \ldots \ldots$ & a2 4 & 13.3 & 85.1 & 8.7 & 83.4 & 9.1 & 465 & 8.6 \\
\hline Now Yrin $\ldots$ & A 883.1 & 36.2 & ค 888.3 & 40.6 & 499.6 & 60.9 & $A 925$ & 588.2 \\
\hline North Carcolma $\ldots . . . . . . . . . . . . . . . .$. & 977 & 71.7 & 988 & 92.8 & 98.9 & 97.1 & 90.1 & $(19.4$ \\
\hline 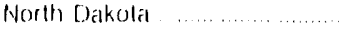 & 76.1 & 294 & 78.4 & 29,5 & 82.5 & 30.4 & 839 & $2 \% .7$ \\
\hline 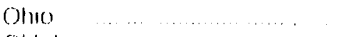 & 860 & 114 & 89.5 & 12.6 & 88.9 & 128 & 89.2 & 20.2 \\
\hline Oklahorna $\ldots \ldots \ldots \ldots \ldots$ & 925 & 485 & 91.3 & 531 & 92.6 & 56.8 & 943 & 57.6 \\
\hline$\ldots \ldots \ldots \ldots \ldots$ & 97.3 & $20 ?$ & 964 & 20.7 & 98.5 & 18.? & $94 . ?$ & 26.6 \\
\hline Penmsylvana $\quad \ldots$ & 796 & 210 & 805 & 235 & 83.3 & 28.4 & 857 & 312 \\
\hline Ahode Island & 1000 & 89.4 & 1000 & 95.1 & 100.0 & 1000 & 100.0 & 100.0 \\
\hline 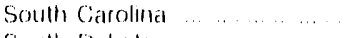 & 97.1 & 76.9 & 97.9 & 76.6 & 98.0 & 856 & 088.3 & $79 ! 5$ \\
\hline South Dakola & 893 & 47.0 & 91.7 & 55.3 & 02.1 & 6.3 .1 & 925 & 76.0 \\
\hline Tennossee $\ldots \ldots \ldots$ & 07.5 & 547 & 98.4 & 60.3 & $963 ?$ & 63.3 & () 18.7 & 65.5 \\
\hline 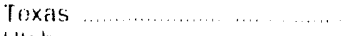 & 94.2 & 21.1 & 89.5 & 27.4 & 940 & 2.7 .5 & 94.6 & 30.2 \\
\hline 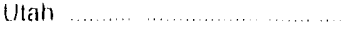 & 100.0 & 19.8 & 1000 & 19.1 & 100.0 & 23.9 & 100.0 & 249 \\
\hline Vermond ... & 100.0 & 100.0 & 1000 & 100.0 & 1000 & 1000 & 100.0 & 100.0 \\
\hline Virginia $\ldots \ldots \ldots \ldots \ldots \ldots$ & 85.8 & 19.9 & 90.1 & 24.0 & 96.6 & 200 & (17.1. & $2 t, 6$ \\
\hline 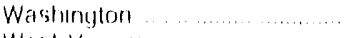 & $9 \% ?$ & 50.9 & $93 \%$ & 61.0 & 95.9 & $6: 3,9$ & $96 . ?$ & 639 \\
\hline 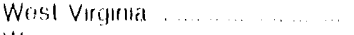 & 55.9 & 10.1 & 601 & 10.8 & 636 & 150 & 70.2 & 176 \\
\hline Wisconsin & 90.7 & 39.2 & 90.8 & 441 & 90.3 & 470 & 00.5 & $56 \theta$ \\
\hline Wyominy & 99.8 & 114 & 90.7 & 169 & 907 & 13.8 & 907 & 19.1 \\
\hline Tolal & 187.4 & 1131.6 & $n 8836$ & 1347 & R 890 & 1936.7 & $\mathrm{H} 90.9$ & 140.6 \\
\hline
\end{tabular}

Soe foolnotos at ond of tables 
Table 33. Percentane of Total Deliveries Represented by Onsystem Sales (Continued)

\begin{tabular}{|c|c|c|c|c|c|c|c|c|}
\hline \multirow[t]{2}{*}{ Slato } & \multicolumn{2}{|c|}{$\begin{array}{l}\text { Tolal } \\
\text { lase9 }\end{array}$} & \multicolumn{2}{|c|}{$\begin{array}{c}\text { Decombor } \\
108 ! 1\end{array}$} & \multicolumn{2}{|c|}{$\begin{array}{c}\text { Novamilior } \\
\text { 1980) }\end{array}$} & \multicolumn{2}{|c|}{$\begin{array}{l}\text { Ootober } \\
\text { lobs }\end{array}$} \\
\hline & Commorcial & Induslatal & Commertial & Inclustrial & Commerclal & Industral & Commorcial & |ncluslial \\
\hline Nlabama $\ldots . . . \ldots \ldots \ldots \ldots$ & 82.9 & 37.4 & 86.6 & 42.4 & 78.3 & 326 & 84.4 & 333.0 \\
\hline 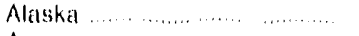 & 100.0 & 565.1 & 1000 & 51.7 & 100.0 & 61.4 & 1000 & 483.0 \\
\hline Arlzonf ............................... & 93.7 & 14.5 & 96.3 & 575 & 94.8 & 560.6 & 01.6 & 44.2 \\
\hline 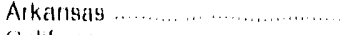 & 931 & 19.1 & 914 & 23.5 & 90.7 & 18.1 & 87.5 & 16.4 \\
\hline Gialiforna & 011.3 & 14.1 & 92.8 & $5 c^{\prime} \cdot 6$ & 001 & $2 ! 5$ & 886.5 & 72.4 \\
\hline Colotido .............................. & 07.3 & 133 & 97.2 & 35.4 & $y(i .4$ & 34.6 & 96.6 & 41.7 \\
\hline Connecticul ......................... & 91.2 & 011.1 & 90.5 & 100.0 & 90.81 & 06.5 & 85.5 & 86.1 \\
\hline Dolaware ................................. & 100.0 & 75.6 & 100.0 & 74.1 & 100.0 & $6(3,9)$ & 100.0 & 64.0 \\
\hline Chstucl of Columbla . ................. & 1000 & 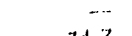 & 100.0 & $\cdots \cdot$. & 100.0 & $\cdots$ & 100.0 & $\cdots$ \\
\hline Floricla $\ldots . . . . . . . . . . . . . . . . . . . . . . . .$. & 100.0 & 74.7 & 100.0 & 74.7 & 100.0 & 683.7 & 100.0 & 72.4 \\
\hline 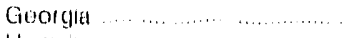 & 002 & 36.4 & 96.8 & 59.6 & 89.4 & 36.4 & 86.4 & 29.6 \\
\hline 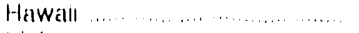 & 100,0 & $\cdots \cdots$ & 100.0 & $\cdots .$. & 100.0 & $\cdots$ & 100.0 & $\cdots$ \\
\hline 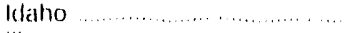 & 87.5 & .5 & 67.4 & 6 & 84.5 & 6 & 70.3 & .3 \\
\hline 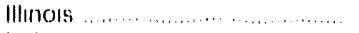 & 693 & 29.6 & 74.5 & 36.9 & 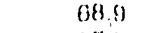 & 30.6 & 64.5 & $? \% .6$ \\
\hline 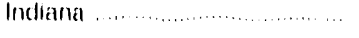 & 95.1 & 21.7 & $(189.9$ & 32.0 & 97.7 & 2.2 .5 & 91.9 & 17.2 \\
\hline lowa & 97.8 & 11.2 & 98.3 & 15.7 & 97.6 & 48.4 & 96.6 & 19.5 \\
\hline 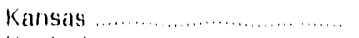 & 0.31 & 22.1 & 92.9 & 26.7 & 88.2 & 10.7 & 88.9 & 22.6 \\
\hline 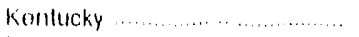 & 94.9 & 30.9 & 97.0 & 41.0 & 94.4 & 21.8 & 00.7 & 20.8 \\
\hline 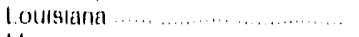 & 99.9 & 17.4 & 99.9 & 40.5 & 99.9 & 42.4 & y9.9 & $4 i j, 4$ \\
\hline 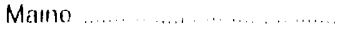 & 1000 & 100.0 & 1000 & 100.0 & 100.0 & 100.0 & 100.0 & 100.0 \\
\hline 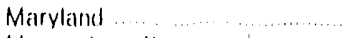 & 96.2 & 326 & 96.83 & 15.7 & 94.6 & 31.5 & 94.2 & 24.3 \\
\hline Matsiachusolls ................. & 999 & 984 & 99.9 & 99.6 & 09.9 & 96.9 & 99.9 & 100.0 \\
\hline 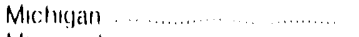 & 70.4 & 17.6 & 73.5 & 22.5 & 69.5 & 15.5 & 64.4 & 12.3 \\
\hline 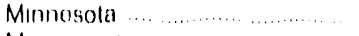 & 958 & 472 & 97.0 & 56.2 & 94.9 & 15.4 & 91.9 & 46.1 \\
\hline Misisissippl $\quad \ldots . . . \ldots \ldots \ldots \ldots \ldots$ & 100.0 & 50.7 & 100.0 & 56.4 & 100.0 & 49.7 & 100.0 & 50.6 \\
\hline 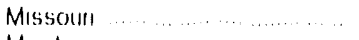 & 92.3 & 47.4 & 92.3 & 48.5 & 90.3 & 47.2 & 88.8 & 41.3 \\
\hline Montana & 98.1 & 737 & 98.1 & 59.2 & 97.9 & 61.3 & 97.5 & 56.63 \\
\hline Nobraskal $\ldots \ldots \ldots \ldots \ldots \ldots$ & 983.4 & 43.3 & 98.5 & $58 . \ddot{*}$ & (9) 8.1 & 60.5 & 98.8 & 76.7 \\
\hline 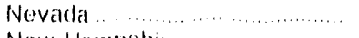 & 96.1 & 11.3 & 98.8 & 13.0 & 07.2 & 7.4 & 962 & 11.2 \\
\hline Now Hampshire ................... & 100.0 & 100.0 & 100.0 & 100.0 & 100.0 & 100.0 & 100.0 & 100.0 \\
\hline 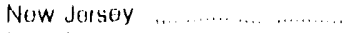 & 978 & 615 & $9 y) 7$ & 83.5 & 99.0 & 71.6 & 94.7 & $53 . \theta$ \\
\hline Now Moxico & 881 & 17.3 & 837.6 & 168 & 85.6 & 13.7 & 86.9 & 16.6 \\
\hline 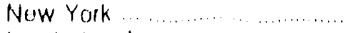 & 85.5 & 397 & 89.7 & 517 & 82.4 & 30.4 & 74.8 & 35.0 \\
\hline North Canoluna ....................... & 935 & 70.1 & 98.1 & 988.0 & 97.1 & 92.1 & 863 & 481.7 \\
\hline North Dakola ............................ & 80.3 & 26.2 & 81.6 & 27.3 & 74.8 & 21.2 & 70.9 & 15.5 \\
\hline Oho $\ldots$ & 86.4 & 160 & 00.3 & 199 & 87.8 & 15.8 & 85.1 & 15.9 \\
\hline 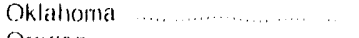 & 80.6 & 50.0 & 91.2 & 61.1 & 89.1 & 56,5 & 86.5 & 46.2 \\
\hline 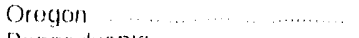 & 98? ? & 219 & 98.0 & 22.1 & 97.3 & 21.8 & 96.7 & 20.8 \\
\hline Ponnsylvanial $\ldots . . . . .$. & $E, 3$ & 29) 1 & 85.3 & 390 & 82.9 & 31.6 & 80.3 & 23.3 \\
\hline 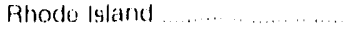 & 83.9 & $82: 1$ & 100.0 & 100.0 & 100.0 & 88.9 & 82.7 & 87.5 \\
\hline Soulh Carolina .................. & 98.1 & 18.5 & 67.3 & 91.3 & 98.0 & 82.9 & 97.8 & 70.5 \\
\hline 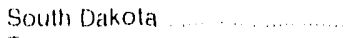 & 92.1 & 606 & $\theta 2.0$ & 630 & 011.1 & 57.8 & 89.6 & 56.4 \\
\hline Tonnessog . . . . . . . . . . & 963.1 & 62.4 & 09.1 & 66.3 & 974 & 62.7 & 97.1 & 61.0 \\
\hline Texas $\quad \ldots \quad \ldots$ & 899 & 25,5 & 92.7 & 24.6 & $\beta 6.9$ & 2.4 .5 & 86.3 & 21.7 \\
\hline Utah $\ldots$ & 1000 & 284 & 100.0 & 23.4 & 100.0 & 23.3 & 100.0 & 22.9 \\
\hline Vermonl & 1000 & 1000 & 1000 & 100.0 & 100.0 & 100.0 & 100.0 & 100.0 \\
\hline . $\quad \ldots \quad \ldots \ldots \ldots$ & 97.1 & 36.3 & 97.9 & 44.6 & 06.1 & 38.7 & 93.3 & 24.8 \\
\hline Washengtor $\quad \ldots \quad$.. $\quad \ldots \ldots$ & 94.0 & 58.6 & 06.5 & 52.9 & 03.6 & 51.0 & 030 & 625 \\
\hline Wesil Virgmia . . ........... & 61.5 & 279 & 71.5 & 34.4 & 58.6 & 26.3 & 19.5 & 22.8 \\
\hline Wisconsin $\ldots \quad \ldots \quad \ldots \ldots$ & $9 \% 6$ & 36.9 & 950 & 580 & 91.1 & 36.6 & 84.3 & 25.0 \\
\hline Wyoming $\ldots \ldots$ & $9(9)$ & 167 & 90.7 & 16.2 & 997 & 11.0 & (6) 6 & 12.9 \\
\hline$\ldots \ldots \ldots$ & 890 & 363 & $90 \%$ & 39.2 & 476 & 34.4 & 35.2 & 37.2 \\
\hline
\end{tabular}

Sog lootnotes at end of tablo. 
Table 33. Percentage of Total Deliveries Represented by Onsystem Sales (Continued)

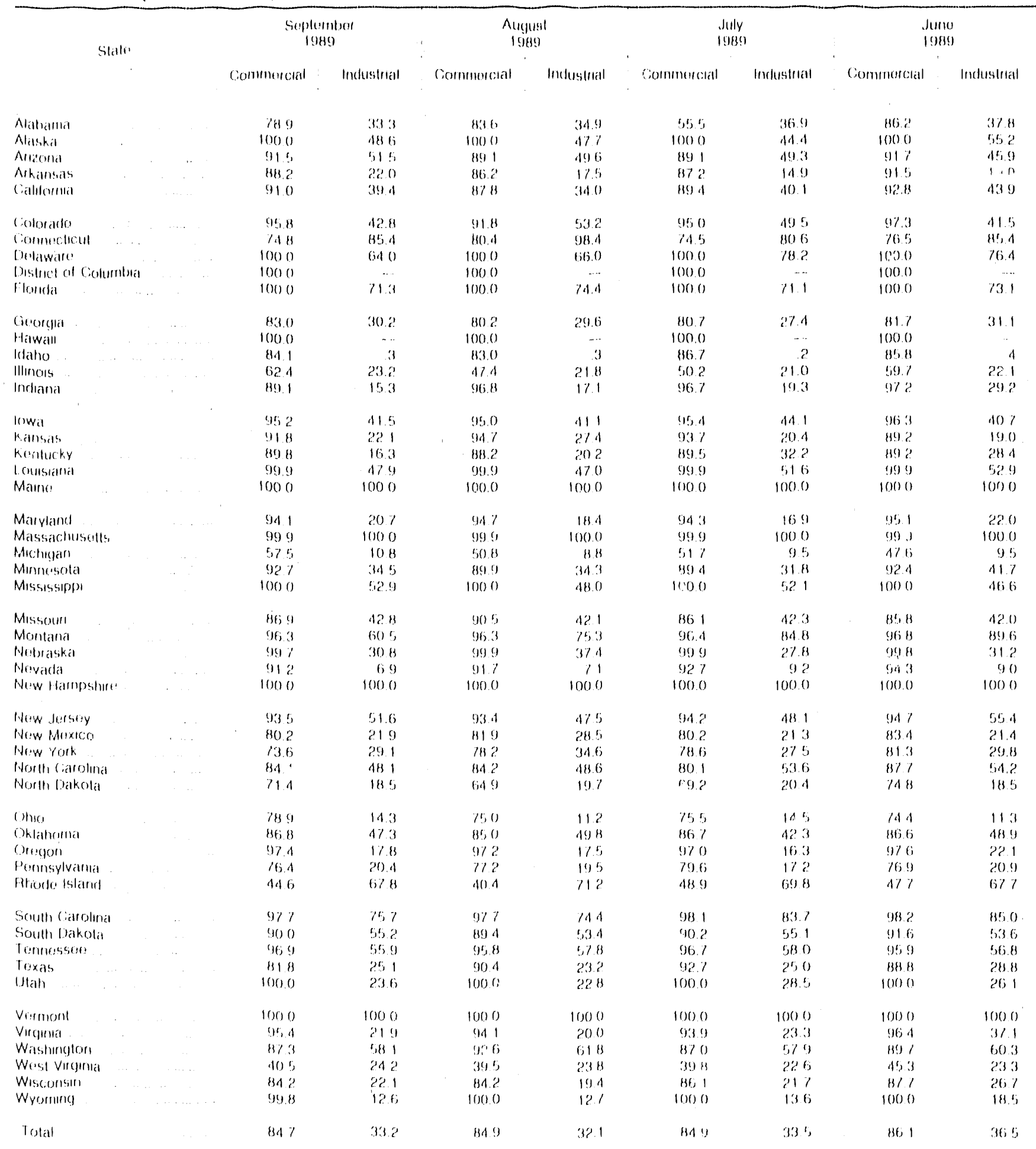


Table 33. Percentage of Total Deliveries Represented by Onsystem Sales (Continued)

\begin{tabular}{|c|c|c|c|c|c|c|c|c|}
\hline \multirow[t]{2}{*}{ State } & \multicolumn{2}{|c|}{$\begin{array}{l}\text { May } \\
1989\end{array}$} & \multicolumn{2}{|c|}{$\begin{array}{l}\text { April } \\
1989\end{array}$} & \multicolumn{2}{|c|}{$\begin{array}{c}\text { March } \\
1989\end{array}$} & \multicolumn{2}{|c|}{$\begin{array}{c}\text { February } \\
1989\end{array}$} \\
\hline & Commercial & Industrial & Commercial & Industrial & Commercial & Industrial & Commercial & Industrial \\
\hline 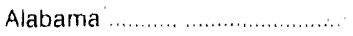 & 83.8 & 37.6 & 90.6 & 39.5 & 89.4 & 42.2 & 82.5 & 40.4 \\
\hline 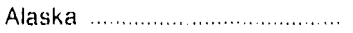 & 100.0 & 60.4 & 100.0 & 50.9 & 100.0 & 61.8 & 100.0 & 59.2 \\
\hline 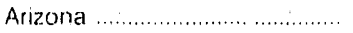 & 92.8 & 45.1 & 93.5 & 43.6 & 94.1 & 31.5 & 96.3 & 32.7 \\
\hline 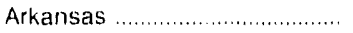 & 91.3 & 18.9 & 94.3 & 17.3 & 95.9 & 18.3 & 95.6 & 23.1 \\
\hline 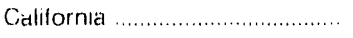 & 94.8 & 38.1 & 88.3 & 39.9 & 93.0 & 37.4 & 95.1 & 50.7 \\
\hline 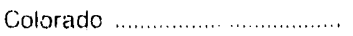 & 97.3 & 42.0 & 97.8 & 43.6 & 98.3 & 39.5 & 98.1 & 46.2 \\
\hline Connecticut ... & 81,6 & 82.6 & 90.0 & 87.2 & 98.4 & 91.7 & 100.0 & 100.0 \\
\hline Delaware .......................... .. .. & 100.0 & 82.9 & 100.0 & 83.8 & 100.0 & 83.5 & 100.0 & 79.2 \\
\hline District of Columbia ..... & $100: 0$ & $-\cdots$ & 100.0 & -- & 100.0 & $\cdots$ & 100.0 & $\cdots$ \\
\hline Florida ....................... & 1000 & 75.9 & 100.0 & 74.0 & 100.0 & 78.0 & 100.0 & 80.0 \\
\hline 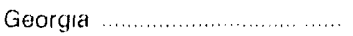 & 85.3 & 30.6 & 88.2 & 33.8 & 89.7 & 37.2 & 93.6 & 42.6 \\
\hline 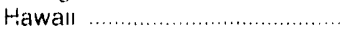 & 100.0 & -- & 100.0 & -- & 100.0 & - & 100.0 & -- \\
\hline 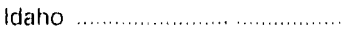 & 82.7 & 4 & 89.0 & .4 & 90.6 & .5 & 90.2 & .7 \\
\hline Illinors & 67.5 & 25.0 & 69.6 & 27.7 & 69.8 & 30.6 & 73.4 & 36.4 \\
\hline 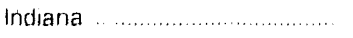 & 92.6 & 14.4 & 92.6 & 17.1 & 94.3 & 20.0 & 93.9 & 28.3 \\
\hline lowa & 97.0 & 43.5 & 98.0 & 40.7 & 98.5 & 44.6 & 98.4 & 46.0 \\
\hline 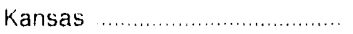 & 92.9 & 18.1 & 94.6 & 21.3 & 94.1 & 22.7 & 94.4 & 22.5 \\
\hline 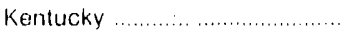 & 91.0 & 31.2 & 94.9 & $31 . \mathrm{J}$ & 96.4 & 32.9 & 96.6 & 35.6 \\
\hline 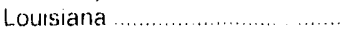 & 99.9 & 53.1 & 99.9 & 51.2 & 99.9 & 17.4 & 99.9 & 45.3 \\
\hline Maine ....................................... & 100.0 & 100.0 & 100.0 & 100.0 & 100.0 & 100.0 & 100.0 & 100.0 \\
\hline Marylan' & 95.9 & 28.1 & 96.7 & 36.3 & 97.1 & 40.6 & 96.6 & 42.5 \\
\hline 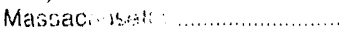 & 99.9 & 100.0 & 99.9 & 100.0 & 99.9 & 100.0 & 99.9 & 91.2 \\
\hline 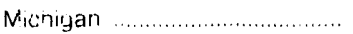 & 64.9 & 16.2 & 71.7 & 18.9 & 76.0 & 23.6 & 74.5 & 23.7 \\
\hline 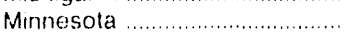 & 95.0 & 48.4 & 95.8 & 52.4 & 97.0 & 53.5 & 97.4 & 56.6 \\
\hline 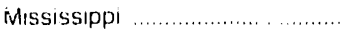 & 100.0 & 50.7 & 100.0 & 47.5 & 100.0 & 55.4 & 100.0 & 54.6 \\
\hline 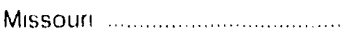 & 90.7 & 44.7 & 93.2 & 47.4 & 94.4 & 53.5 & 93.9 & 53.4 \\
\hline Monterla & 97.4 & 85.4 & 98.6 & 83.7 & 98.8 & 88.9 & 98.9 & 89.9 \\
\hline Nebraska & 99.7 & 37.5 & 99.8 & 42.2 & 97.1 & 41.9 & 96.5 & 45.9 \\
\hline Nevada & 94.2 & 8.9 & 95.0 & 11.2 & 96.9 & 15.4 & 98.1 & 21.3 \\
\hline New Hampshire .......................... & 100.0 & 100.0 & 100.0 & 100.0 & 100.0 & 100,0 & 100.0 & 100.0 \\
\hline 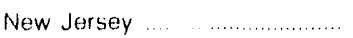 & 96.2 & 53.3 & 98.3 & 58.7 & 98.7 & 62.6 & 58.9 & 75.1 \\
\hline New Mexico & 86.6 & 28.6 & 86.5 & 15.9 & 91.4 & 2.2 & 80.2 & 10.9 \\
\hline 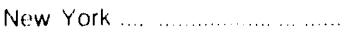 & 85.4 & 35.5 & 85.0 & 36.3 & 89.3 & 45.7 & 90.1 & 47.3 \\
\hline 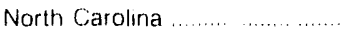 & 89.6 & 57.6 & 92.4 & $71:$ & 94.9 & 78.1 & 98.9 & 96.8 \\
\hline 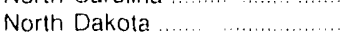 & 80.6 & 22.0 & 83.9 & 34.9 & 84.0 & 33.4 & 84.8 & 36.5 \\
\hline Ohwo & 81.2 & 12.7 & 86.0 & 15.3 & 87.1 & 16.6 & 88.1 & 18.7 \\
\hline Oklahoma & 89.1 & 47.1 & 87.9 & 46.7 & 91.7 & 48.1 & 90.2 & 54.7 \\
\hline Oregon & 97.6 & 19.4 & 98.3 & 22.2 & 98.7 & 24.6 & 99.2 & 31.9 \\
\hline 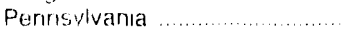 & 81.7 & 23.9 & 874 & 25.5 & 88.7 & 33.8 & 88.9 & 42.9 \\
\hline 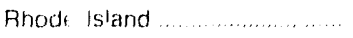 & 83.9 & 71.3 & 87.1 & 82.1 & 100.0 & 100.0 & 100.0 & 100.0 \\
\hline 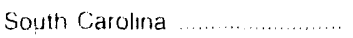 & 93.1 & 67.7 & 98.3 & 75.8 & 98.6 & 74.5 & 98.5 & 76.5 \\
\hline 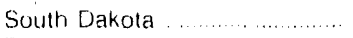 & 92.5 & 56.3 & 92.1 & 65.5 & 92.8 & 68.1 & 93.1 & 68.6 \\
\hline 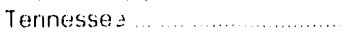 & 968 & 67.4 & 97.5 & 61.9 & 98.9 & 66.1 & 98.9 & 65.9 \\
\hline 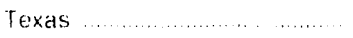 & 89.9 & 25.6 & 91.9 & 26.4 & 93.2 & 28.1 & 91.8 & 25.4 \\
\hline 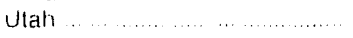 & 100.0 & 28.8 & 100.0 & 31.3 & 100.0 & 30.3 & 100.0 & 38.8 \\
\hline Vermont & 100.0 & 100.0 & 100.0 & 100.0 & 00.0 & 100.0 & 100.0 & 100.0 \\
\hline Virgınıa ... . . . . . . . . & 964 & 28.3 & 97.1 & $45 ;$ & 98.1 & 43.3 & 98.4 & 51.0 \\
\hline 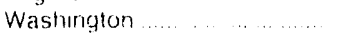 & 93.0 & 575 & 93.2 & 60.9 & 96.9 & 58.9 & 94.8 & 62.4 \\
\hline 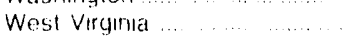 & 560 & 233 & 63.9 & 30.8 & 65.3 & 27.1 & 69.0 & 36.6 \\
\hline Wisconsin & 92.6 & 32.8 & 94.0 & 35.7 & 94.5 & 40.9 & 94.2 & 43.5 \\
\hline Wy'sming & 100.0 & 24.5 & 100.0 & 15.5 & 100.0 & 21.5 & 100.0 & 21.7 \\
\hline Total & 88.2 & 35.3 & 89.1 & 36.1 & 90.8 & $37:$ & 91.2 & 39 \\
\hline
\end{tabular}

Seet footinotes at end of table 
Table 33. Percentage of Total Deliveries Represented by Onsystem Sales (Continued)

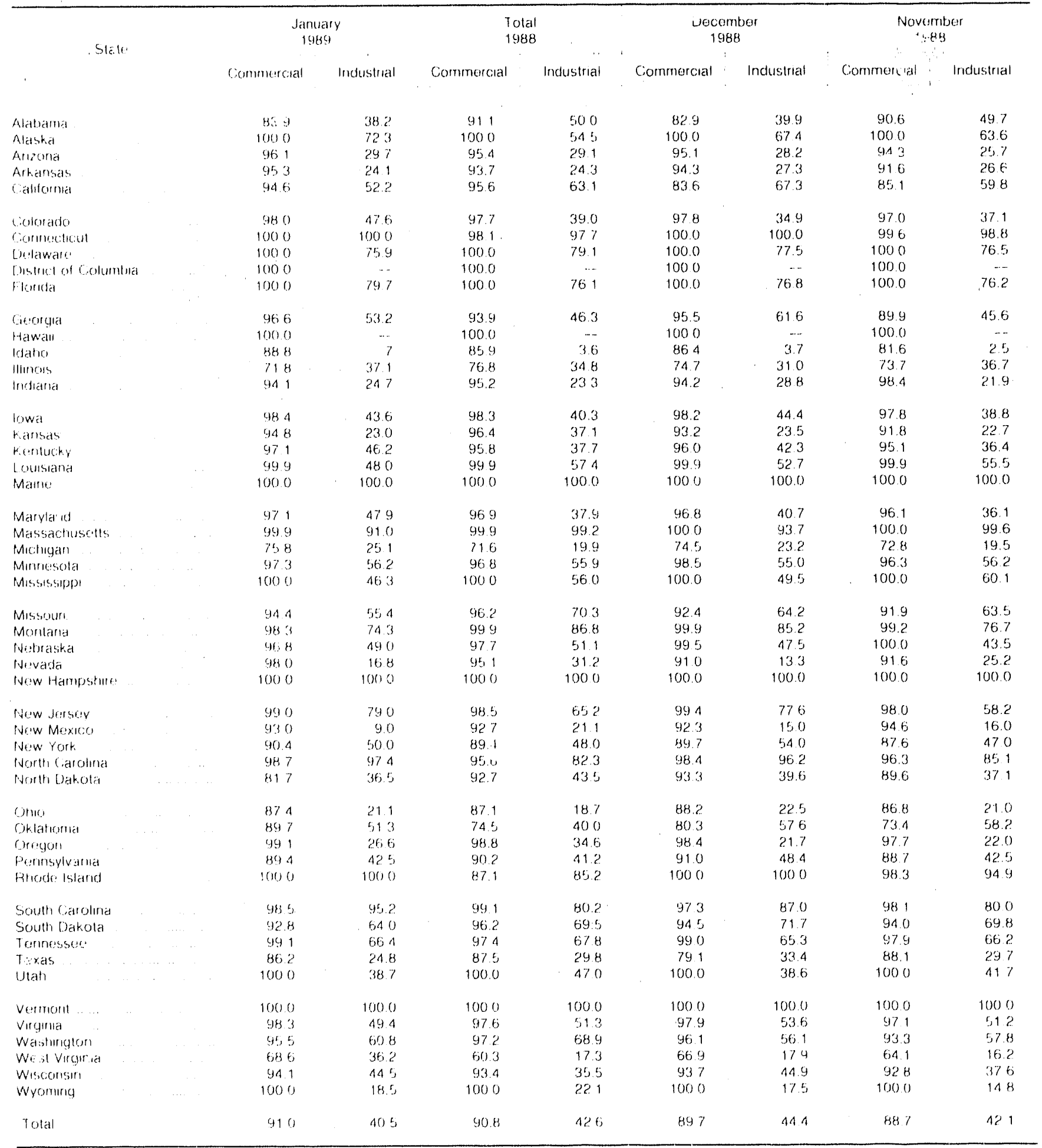

H Revised lotas

Nol Applicatule

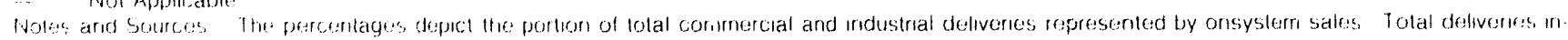
cturdo volume transported to commencial and iridustral comsumers for the accourit of others 
Figure 7. Percentage of Total Deliveries Represented by Onsystem Sales

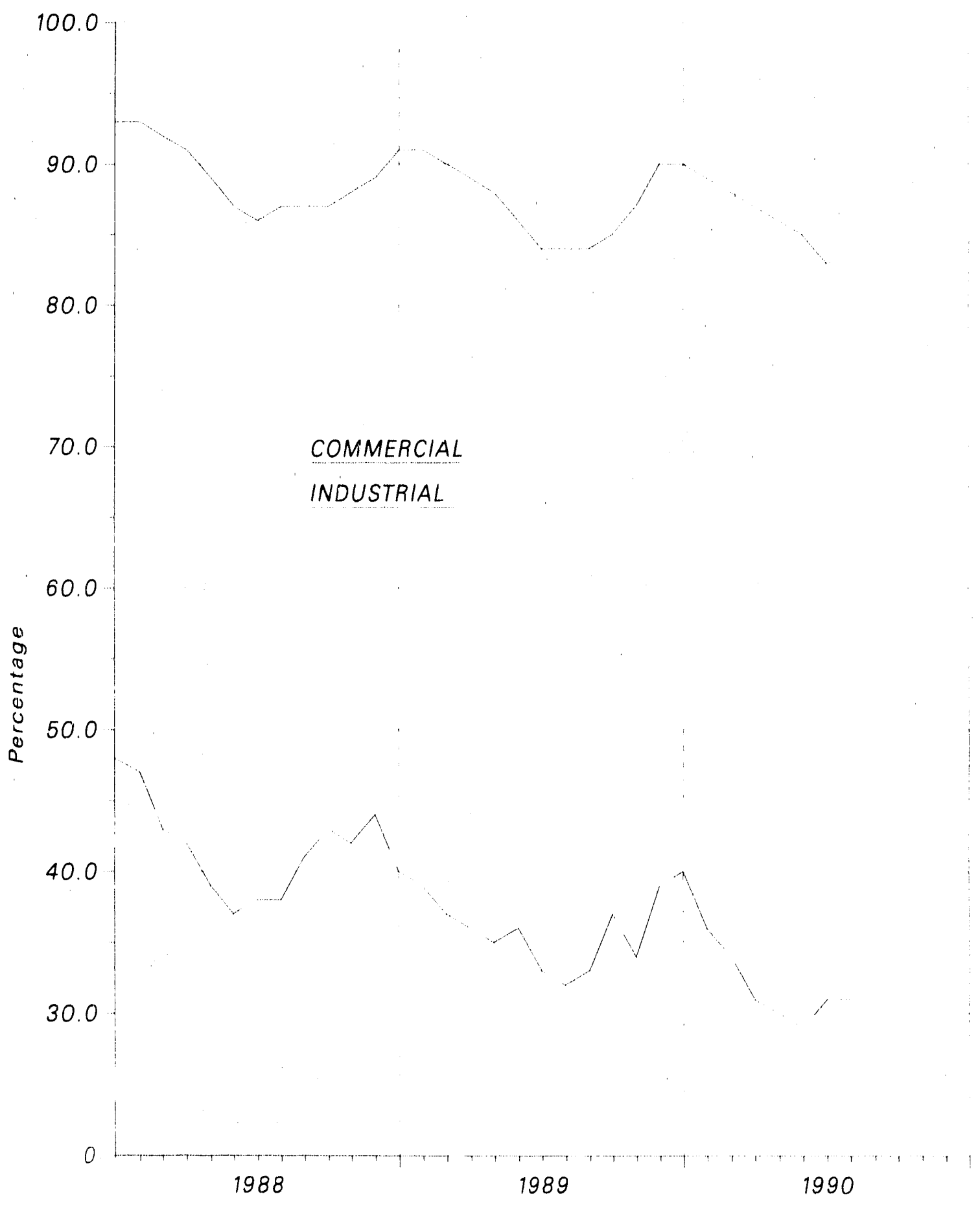

Source: Form ElA-857. 


\section{Table Notes and Sources}

\section{Table 1}

Notes: Data for 1984 through 1989 are final. All other data are preliminary unless otherwise indicated. Geographic coverage is the 50 States and the District of Columbia. Totals may not equal sum of components because of independent rounding.

Sources:

- EIA, Natural Gas Annual 1989. Table 7 and EIA estimates, January 1990 through current month. See Explanatory Notes 1, 3, and 6 for discussion of computation, estimating procedures, and revision policy.

\section{Table 2}

Notes: Data for 1984 through 1989 are final. All other data are preliminary unless otherwise indicated. Geographic coverage is the 50 States and the District of Columbia. Totals may not equal sum of components because of independent rounding.

Sources:

- Total Dry Gas Production: ElA Natural Gas Annual 1989, 1984 through 1989; IOCC, MMS reporting, and ElA estimates, January 1990 through current month. See Explanatory Note 3 for estimation procedures and revision policy.

- Withdrawals from and Additions to Storage: EIA Natural Gas Anmual 1989, 1984 through 1989; Form FERC-8 and Form ElA-191, January 1990) through current month.

- Supplemental Gaseous Fuels: ElA Natural Gas Annual 1989.1984 through 1989; and EIA computations, January 1990 through current month. See Explanatory Note 2 for discussion of eomputation procedures and revision policy.

- Imports and Exports: Form FPC-14, 1984 through 1989: and EIA estimates, January 1990 through the current month. See Explanatory Note 4 for discussion of procedures and revision policy.

- Consumption and Unaccounted For: EIA Natural

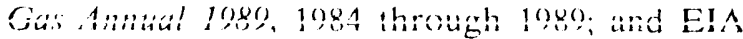
computations, January 1990 through current month. See Explanatory Notes 5 and 11 for discussion of computation procedures and revision policy

\section{Table 3}

Notes: Data for 1984 through 1989 are final. All other data are preliminary unless otherwise indicated. Geographic coverage is the 50 States and the District of Columbia. Totals may not equal sum of components because of independent rounding.

Sources:

- All data except electric utility: EIA Natural Gas Annual 1989, 1984 through 1989; and Form ElA-857 and computations January 1990 through the current month. See Explanatory Note 5 for computation procedures and revision policy. Electric utility data: Form ElA-759, "Monthly Power Plant Report" (formerly Form FPC-4).

\section{Table 4}

Notes: Data for 1984 through 1989 are final. All other data are preliminary unless otherwise indicated. Geographic coverage is the 50 States and the District of Columbia. Average prices for gas delivered to industrial consumers for 1984 through 1987 include imputed averages for volumes of gas delivered for the account of others. From 1988 on, average prices reflect onsystem sales prices only. The change in series in 1987 affects the commercial, industrial, and all consumers averages.

\section{Sources:}

- Average wellhead price: EI A Natural Gas Annual 1989, 1984 through 1989; and EIA estimates, Janwary 1990 through current month. See Explanatory Note 8 for estimation procedures and revision policy.

- Imports and Interstate Pipeline Company Purchases: Form FERC-11.

- Average City Gate, Residential, Commercial and Incistrial average prices for 1984 through current month from Form EIA-857. See Explanatory Note 5 for discussion of revision policy.

- Earlier prices from EIA Natural Gas Anmual 1989. Electric Utilities averages from Form FERC-423, "Monthly Report of Cost and Quality of Fuels for Electric Plan's."

\section{Table 5}

Notes: See Explanatory Note 12 for discussion of purchased gas adjustment filings. Totals may not equal sum of components because of independent rounding.

Source:

- Purchased Gas Adjustment filings for interstate pipeline companies. 


\section{Table 6}

Notes: Data for 1985 through 1989 are final. All other data are preliminary.

\section{Sources:}

- Form FPC-14, "Annual Reporl for Importers and Exporters of Natural Gas."

- "Quarterly Sales and Price Report" prepared by the Office of Fuels Programs, Office of Fossil Energy.

\section{Table 7}

Notes: Data for 1984 through 1989 are final. All other data are preliminary unless otherwise indicated. Totals may not equal sum of components because of independent rounding. See Explanatory Notes 1 and 3 for discussion of computation procedures and revision policy.

\section{Solurces:}

- Ela Natural Gas Allnual 198\%, 1984 through 1989.

- IOCC, MMSreports, and EIA computations, January 1990) through current month.

\section{Table 8}

Notes: Data for the current month and the previous 2 months are preliminary. All other dala are final. Dates shown indicate date received at the FERC. Data include affirmative determinations only. See Explanatory Note 10 for discussion of Title I of the Natural Gats Policy Act. Geographic eoverage is the 50 States and the District of Columbiat. Totals may not equal sum of components because of independent rounding.

Source:

- Furm FERC-121.

\section{Table 9}

Notes: Dates shown indicate date received at FERC. Values include affirmative determinations only. See Explantary Note 10 for discussion of Title I of the Natural Gas Policy Act. Geographic coverage is the 5() States and the District of Columbia. Totals may not equal sum of components hecause of independent rounding.

Sourse:

- Form FERC-121.
Table 10

Note: Data shown arc based on filings received al FERC during the month indicated by the table heading. Data include affirmative determinations only. See Explanatory Note 10 for discussion of Title I of the Natural Gats Policy Act. Geographic coverage is the 50) States and the District of Columbia. Totals may not equal sum of components because of independent rounding.

Source:

- Form FERC-121.

\section{Table 11}

Notes: Geographic coverage is the 50) States and the District of Columbia. A "small producer" is one with ammal sales subject to FERC jurisdiction not in excess of 11) hillion cubic feet. On November 16, 1984, the Federal Energy Regulatory Commission issued Order No. 406 in Docket No. RM 84-14-000 providing for inclusion of miximum lawful prices under Sections 103(b)(2) and 105(b)(3) of the Natural Gas Policy Act. Order No. 406 becanie effective on January 1, 1985.

Source:

- Federal Register. "Rules and Regulations."

\section{Table 12}

Notes: Data up to 3 years prior to current month are final. All other data are prelininary unless otherwise indicated. See Explanatory Note 9 for discussion of major interstate pipeline companies. Totals may not equal sum of conponents hecause of independent rounding. This table shows selected items only and therefore does not balance mathematically.

Source:

- Form leter-11

\section{Table 13}

Notes: The summaries presented in this table are exclusive of transactions between major pipeline companies in the computation of total pipeline activities to eliminate double-counting. Data up to 3 years prior to current month are final. All other data are preliminary unless otherwise indicated. See Explanatory Noteg for discussion of major interstate pipeline companies. Totals may not equal sum of components because of independent sounding.

Source:

- Form Fl:R(-11. 


\section{Table 14}

Notes: Two lines have been added to this table to explicitly differentiate transactions between major and nommajor pipeline companies. Totals may not equal sum of components due to independent rounding and provisions for pending regulatory adjustments,

Source:

- Form FERC-11.

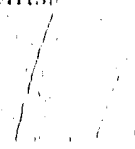

\section{Table 15}

Notes: Previously published manufactured gas is now summarized with liquefied natural gas, gasified coal, and synthetic gas production. Also, the summaries presented in this table are exclusive of transactions between major pipeline companies in the computation of total pipeline activities to eliminate double counting. See Explanatory Note 9 for discussion of major interstate pipeline companies. Totals may not equal sum of comporents because of independent rounding.

Source:

- Form FERC-11.

\section{Table 16}

Notes: Previously published manufactured gas is now summarized with licjuefied natural gas, gasified coal, and synthetic gas production. Two lines have been added to this table that explicitly differentiate transactions between major and nonmajor pipeline companies. See Explanatory Note 9 for discussion of major interstate pipeline companies. Totals may not equal sum of components because of independent rounding.

Source:

- Form FERC-11.

\section{Table 17}

Notes: Data for 1984 through 1989 are final. All other data are preliminary unless otherwise noted. See Explanatory Note 7 for discussion of revision policy. Gas in storage at the end of a reporting period may not equal the quantity derived by adding or subtracting net injections or withdrawals during the period to the quantity of gas in storage at the beginning of the period. This is due 10 changes in the quantities of native gas included in base gas and/or ineses in base gas due to migration from storage reservoirs. Totals may not equal sum of components because of independent rounding. Geographic coverage in the so stutes and the District of Columbia.
- Form EIA-191, Form FERC-8, and Form EIA-176.

\section{Table 18}

Notes: Data for 1984 through 1989 are final. All other data are preliminary unless otherwise noted. See Explanatory Note 7 for discussion of revision policy. Gas in storage at the end of a reporting period may not equal the quantity derived by adding or subtracting net injections or withdrawals during the period to the quantity of gas in storage at the beginning of the period. This is due to changes in the quantitie. of native gas included in base gas and/or losses in base gas due to migration from storage reservoirs. Totals may not equal sum of components because of independent rounding. Geographic coverage is the 50 States and the District of Columbia.

\section{Sources: \\ - Form EIA-191, Form FERC-8, and Form ElA-176.}

\section{Table 19}

Notes: Data for 1984 through 1989 are final. All other data are preliminary unless otherwise noted. See Explanatory Note 7 for discussion of revision policy. Gas in storage at the end of a reporting period may not equal the quantity derived by adding or subtracting net injections or withdrawals during the period to the quantity of gas in storage at the beginning of the period. This is due to changes in the quantities of native gas inclucied in base gas and/or losses in base gas due to migration from storage reservoirs. Totals may not equal sum of components because of independent rounding. Geographic coverage is the 50 States and the District of Columbia.

Sources:

- Form EIA-191, Form MERC-8, and Form EIA-176.

\section{Table 20}

Notes: Gas in storage at the end of a reporting perood may not equal the quantity derived by adding or subIracting net injections or wit hdrawals during the period to the quantity of gas in storage at the beginning of the period. This is due to changes in the quantities of native gas included in base gas and/or losses in base gas due to migration from storage reservoirs. Totals may not equal sum of components because of independent rounding. Geographice coverage is the 50 Stare: and the District of Columbia. 
- Form FERC-8.

\section{Table 21}

Notes: Gas in storage at the end of a reporting period may not equal the quantity derived by adding or subtracting net injections or withdrawals during the period 10 the quantity of gas in storage at the beginning of the period. This is due to changes in the quantities of native gas included in base gas and/or losses in base gas due to migration from storage reservoirs. Totals may not equal sum of components because of independent rounding. Geographic coverage is the 50 States and the District of Columbia.

Sources:

- Form FERC-8 and Form EIA-191.

\section{Tables 22-25}

Notes: Geographic coverage is the 50 States and the District of Columbia. Volumes in these tables represent the amount of natural gas delivered to four groups of natural gas consumers. See Explanatory Note 5 for discussion of computations and revision policy.

Sources:

- Residential, Commercial, and Industrial Volumes: Form EIA-857.

- Electric Utility Volumes: Form EIA-759.

\section{Table 26}

Notes: Geographic coverage is the 50 States and the District of Columbia. Volumes in this table represent the amount of natural gas delivered to all consumers. See Explanatory Note 5 for discussion of computations and revision policy.

Source:

- Form EIA-857 and Form EIA-759.

\section{Table 27}

Notes: Geographic coverage is the 50 States and the District of Columbia. Prices in this table represent the average price of natural gas by State at the point where the gas is transferred from a pipeline 10 a local distribution company within the State. See Explanatory
Note 5 for discussion of computations and revision policy.

Source:

- Form ElA-857.

\section{Tables 28-31}

Notes: Data for 1988 and 1989 are final. All other data are preliminary unless otherwise indicated. Geographic coverage is the 50 States and the District of Columbia. Average prices for gas delivered to commercial and industrial consumers for 1988 forward reflect onsystem sales prices only. See Explanatory Note 5 for discussion of computations and revision policy. See Table 33 for data on onsystem sales expressed as a percentage of both total commercial and total industrial deliveries.

Sources:

- Residential, Commercial, and Industrial Prices: Form EIA-857.

- Electric Utility Prices: Form FERC-423.

\section{Table 32}

Notes: Geographic coverage is the 50 States and the District of Columbia. Prices in this table represent the on-system average price of natural gas delivered to all consumers. See preceding note regarding break in average price series. See Explanatory Note 5 for discussion of computations and revision policy.

Sources:

- Form EIA-857 and Form FERC-423.

\section{Table 33}

Notes: Volumes of natural gas reported for the commercial and indust rial sectors in this publication include data for both sales and deliveries for the account of others. This table shows the percent of the total State volume that represents natural gas sales to the commercial and industrial sectors. This information may be helpful in evaluating commercial and industrial price data which are based on sales data only. See Appendix C, Statistical Considerations, for a discussion of the computation of natural gas prices.

Source:

- Form EIA-857. 


\section{Appendix A}

Explanatory Notes 


\section{Appendix A}

\section{Explanatory Notes}

\section{Note 1. Nonhydrocarbon Gases Removed}

\section{Annual Daia}

Data on nombedrocarbon gases removed from marketed production--antbon dioxide, helium, hydrogen sultide, and nitrogen--are reported by Stale agencies on the voluntary Form EIA-627. For 1989, of the 32 producing States, 22 reported data on nombydrocarbon gases removed. The 22 States accounted for 57 perecent of total 1989 gross withdrawals. Of the 22 States reporting nonhydrocarbon gases removed, 11 reported zero values: Alaska, Arizona, Arkansas, Illinois, Indiana, Maryland, Missouri, New York, Oregon, South Dakota, and Virginia. The nine Stales reporting volumes greater than zero are Alabama, Califormia, Colorado, Florida, Mississippi, New Mexico, North Dakota, Texas, and Wyoming. Two States (Kentucky and Nebraska) reported quantities unknown but considered insignificant. In addition, Kansas, Louisiana, Montana, and Oklahoma, which together accounted for 38 percent of gross withdrawals, did not repont nonhydrocarbon gases removed separately. However, their gross withdrawal clata excluded all or most of the nonhydrocarbon gases removed on leases. No estimates are made for states not reporting nombydrocarbon gases rernoved.

\section{Preliminary Monthly Data}

All monthly data are considered prelimis ary until after publication of the Natural Gas Anmual for the year in which the report month falls. Three States report monthly data on nombedrocarbon gases removed: Alabama, Texas, and Mississippi. Monthy data for California, Colorado, Florida, New Mexico, North Dakota, and Wyoming are estimated based on annual data reported on Form ElA-627. Nombydrocarbon gasc's as an annual pereentage of gross withdrawals reported by each of the six States is applied to each State's monthly gross withdrawal data to produce all estimate of nombedrocarbon gases removed.

\section{Final Monthly Data}

Monthly data are revised after publication of the Natural Gas Annual by proportionally allocilling the differences between anmual data reported on the form EIA-627 and the sum of monthly data (JantuaryDecember).

\section{Note 2. Supplemental Gaseous Fuels}

\section{Annual Data}

Annual dala are published from Form EIA-176.

\section{Preliminary Monthly Data}

All monthly data are considered preliminary until after the publication of the Natural Gas Annual for the year in which the report month falls. Monthly estimates are based on the annual ratio oi supplemental gaseous fuels to the sum of dry gas production, net imports, and net withdrawals from storage. This ratio is applied to the monthly sum of these three clements to compute a monthly supplemental gaseous fuels figure.

\section{Final Monthly Data}

Momthly data are revised after publication of the Natural Gas Annual. Final monthly data are estimated based on the revised annual ratio of supplemental gascous fucls to the sum of dry gas production, net imports, and net withdrawals from storage. This ratio is applied (o) the revised monthly sum of these three clenents to compute final monthly data. 


\section{Note 3. Production}

\section{Annual Data}

Nallural gars produclion dala are colleceled from 32 gatsprofucing Stales on lonrm l:1 A-627. The U.S. Minceals Management Service (MMS) alser supplices dala on llac

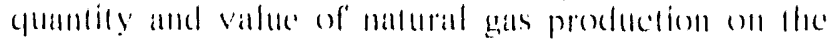

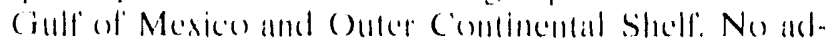
justments are made to the datal

\section{Estimated Monthly Data}

Shate markeded prodection data for a particular momb

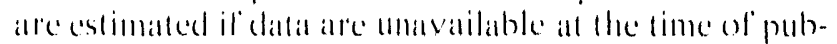
lications. The dallat alle estamalted based on an average

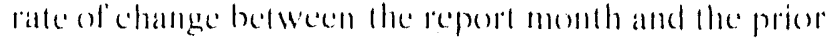
month ovel the previous a years. The lalle-ol-change pereentage is applied to the data for the momth prior to the repert momth on compute the estimate for the report momb in the current year.

Lstimaten for total U.S. matreled production are based on he application of historical (batest 3 calcondar years) month-lo-momth ratios of daily prodection rates to the latest pretimimaty reported mombly prodection data. Slate estimales for momhydrocarbon gas removed, gas ieced for repressuring. and gals vented and hared are based on the ratio of the latest prediminary reported 3-month average of these catcegeries lo matkeled poreduction. These ration alle applied to the estimates of matreked production lo colculale figures for mombydrocathem gares removed, gas used for repressuring, and gats vented and flared. Fstimates for geross withdrawal data alle the sum of the estimates cal-

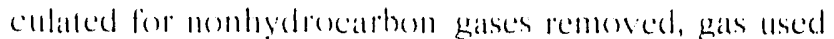
for repressuring, gats vemed and flared, and marketed production

\section{Preliminary Monthly Data}

All monthly dala are censidered preliminary until aller

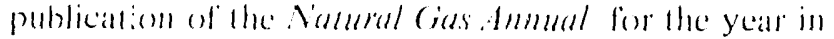
which the repont month balls. Peeliminary monthy datat are published frem reports from the Interstate oil Compact Commission ( $(1) C^{\circ} \mathrm{C}$ ) and the MMS.Volumetric dalla arecomverled, an mecessary, 10 a standard 14.73 psia pressure base. Data are revised as Table 7 monthly data are 11 related.

\section{Final Monthly Data}

The differences helween each slate's anmeal produc-

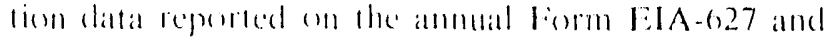

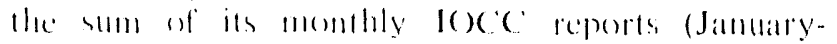

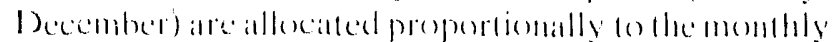
Io) (latlit.

\section{Note 4. Imports and Exports}

\section{Annual Data and Final Monthly Data}

Annual and final monthly dalu are published from the

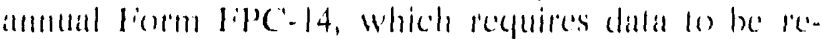
ported by month fore the calcendar yeatr.

\section{Preliminary Monthly Data - Imports}

Preliminary monthly import dilla are based on dala firour the National Energy Band of Camala and responses lo informal industry contaces and lila estimates. Predimbary data are revised after the publicestion of the arlicle "U.S. I mporls and Exports ol Natural (ias" for llec calcolall year.

\section{Preliminary Monthly Data . Exports}

Prefiminary monthly export dalal are hased on historical data firem the form lipe - 14, informal industry contats:s, and information s,athered from natural gas industry trade publications. Preliminary monthly data are revised afler publication of "U.S. Imports and IExports of Nallural Cias" for Hhe callendar year in which the report month falls.

\section{Note 5. Consumption}

\section{All Annual Data}

All comsumption dala except electric mility dala alle

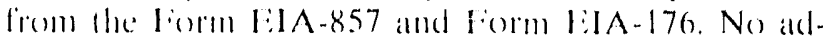
justments are made to the dalta. Bilectrice utility data are reported on Form rilA-75\%.

\section{Monthly Data}

All momhly data are comsidered preliminary umbil after publication of the Nantral (ias dumbul.

\section{Total Consumption}

\section{Preliminary Monthly Data}

The most current month estimate is ralculated based on the aribmetic average change from the previous momb for he previous ? years. The following month this estimalle is revined by smmming the e(momponemts (pipeline fied. lease and plant linel, andel deliveries fo comstimlexs) 


\section{Final Monthly Data}

Monthly data ure revised after publication of the Natural Gass Ammual. Final monthly total consumprion is obtained by summing its components.

\section{Residential, Commercial, and industrial Sector Consumption}

\section{Preliminary Monthly Data}

Preliminary monthly residential, commercial, and industrial data are from Form EIA-857. See Appendix C. "Statistical Considerations," for a detailed explanation of sample selection and estimation procedures.

Price data are representative of prices for gas sold and delivered to residential, commercial, and industrial eonstumers. These prices do not reflect average prices of natural gas transported to consumers for the aceount of third partices or "spot-market" prices.

\section{Final Monthly Data}

Monthly data are revised after the publication of the Natural Gas Annual. Final monthly data are estimated by allocating annual consumption data from the Form E1A-176 to each mont. proportion to monthly sales volumes reported in Form EIA-857.

\section{Electric Utility Sector Consumption}

\author{
All Monthly Data \\ Monthly data published are from form EIA-759.
}

\section{Pipeline Fuel Consumption}

\section{Preliminary Monthly Data}

Preliminary data are estimated based on the pipeline fuel consumption as an annual percentage of total consumption from the previous year's F.orm EIA-176. T'his percentage is applied ro each month's lotal consumption figure to compute the monthly estimate.

\section{Final Monthly Data}

Monthly data ate revised after the publication of the Natural Cias Ammual. Final monthly data are based on the revised annual ratio of pipeline fuel consumption
(1) fotal comsumption from the form bis-176, This ratto is applicel to cach month's revised total contsumption figme to compute linal monthly pipeline fied contsumbifion estimales.

\section{Lease and Plant Fuel Consumption}

\section{Preliminary Monthly Data}

Preliminney monthly data are estimated based on lease and plant fuei consumption as an annual pereentage of matreted production excluding nonhydrocarbon gases). This fiencentage is applied to ench month's marketed production ligure to compule estimated lease and plant fuel consumprion.

\section{Final Monthly Data}

Monthly data are revised after publication of the Natural Gass Annual. Final monthly data are based on a revised annual ratio of lease and plant fiscl consumption to marketed production (excluding nonhydrocarbon gatses) from form EIA-176. This ratio is applied to each month's revised marketed production figure to compute final monthly lease and plant fuel consumption estimates.

\section{Note 6. Extraction Loss}

\section{Annual Data}

Extraction loss data are calculated from filings of Form EIA-64A, "Annual Report of" the Origin of Natural Gan Liquids Production." For a fuller discussion, see the Natural Gas Annuml 1989.

\section{Preliminary Monthly Data}

Preliminary data are estimated based on extraction loss ats an annual percentage of marketed production. This pereentage is applied to each month's marketed production to estimate monthly extraction loss.

\section{Final Monthly Data}

Monthly data are revised after the publication of the Netural (iass Anmual. Final momblhy data are estimated by allocitting anmual extraction less datta to cach month batsed on its lotal matural gats disposition. 


\section{Note 7. Natural Gas Storage}

\section{Underground Natural Gas Storage}

All monthly data concerning undergerennd solorage are published from the essentially identical forms,

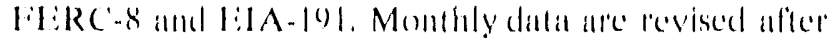
publiculion of "Undergenend Natural Gas Slormge in the United States" for Hhe healting yeare (A pril through March) in which the report month fulls. In addition, injection and withdrawal data from the FERC-8/ BIA-19) survey are adjusted fo corresipond (o) data form lorm E:1A-176 following publication of the Nat-

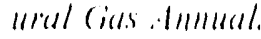

\section{Underground and Liquefied Natural Gas Storage}

The fintil monthly and ammual storinge and withdrawal data for 1084t dherengh 1089) shown in Tiable 2 inclucle

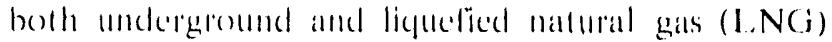
storage. Underground sterage dalla atre oblatined from the HER(-8/I:IA-19) and I:IA-176 surveys in the manner described carlier. Anmual data on I.NG addidions and wilhdrawals ane taken from form EIA-176. Monthly datta ate estimated by computing the rattion of each month's underground storage addetitens and withdrawals to annual undergeround storigge additions and withdrawals and applying it (o) antulail I.NG data.

\section{Note 8. Average Wellhead Value}

\section{Annual Data \\ form b:IA-627 requests Stalle agencies for repore the quantity and value of marketed production. When com- plete data are anavailable, the form instructs the State agency to report the availathe value and the quantity of marketed production associaled with this value. A number of states reported volumes of production and associated values for ofloer than markeled production. In addition, infermation for several States which were molable to provide dilla wals obtatined from form EIA-176. It should be noled that Form EIA-176 reports a fraction of state production. The imputed value of marketed predection in eact state is calculated hy di- viding the State's reported vilue hy its assiceciated well- head value. This unit price is then applied to the quant- tity of the Stalle's marketed preslection to derive the impuled vilue of matreterf productions.}

\section{Preliminary Monthly Data}

All estimate of the U.S. gats price is mate canch month

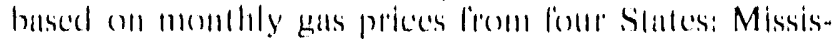
sippi, Now Mexico, oklahloman, and lexus.

\section{Final Monthly Data}

Preliminury monthly data are revised alfere the publi-

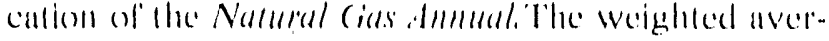
age 12-montla pricese for 'lexas, New Mexico, ()klaheman, atnd Mississippi alde compured to the wellhead

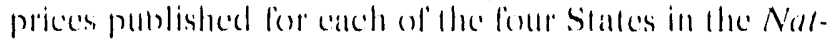
ural cias atumual: The ratio derived from this compari-

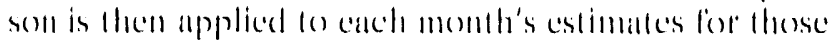
slates, and the monthly data are revised.

\section{Note 9. Financial Data of Major Interstate Pipeline Companies}

The prices in Thable 4 for imports and purchases forom producers, and indestrial sales by major interstate piperlince compantes, and all datit in Tiable's 12 through 16

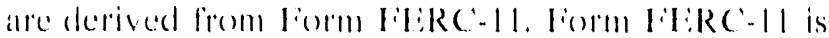
filed monthly by dhe approximately 47 major interstate natural gas pipeline companies. A major pipeline com pany is clefined as anse "whose combined salles for re.-

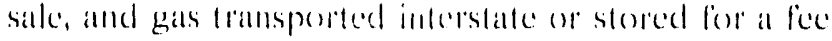
exceceded 50 billion cubic fied in the previous callendaus yciar."

Data reported by the major interstate pipeline compa-

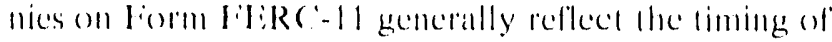

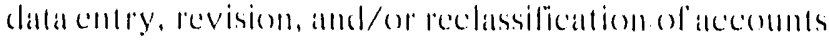
in the compantes' aceounting records in alcerordance with the FITRC regulations and regulatory filings. Corlain dala may also be estimated. Consecguently, the data reported and showen in lables 12 lherough to for any given month maty include or reflect oul-of-period dollar or volume addustments, restatements or revisions, or

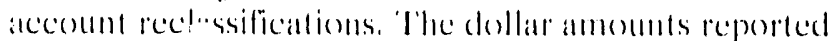
als paid or received and volume. reported ats delivered or received maly also include anounts paicl, dedivered, or received moler contractual provisions such as prepayment, takce-or-pay, minimum lakce, or minimum bill

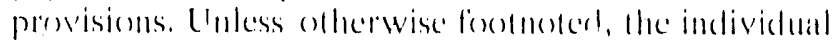
dalta items, compuled averages, and aggeregalled lotals shown include the effect of any and all stech adjustments, revisions, estimates, rectissificiations, ande/or contractual provisions. Average prices are not reported (111) the forem 11. The averages shown ane computed hy dividing the tetal dollats reported for the particular item by the letal velume reperted for the same item. 


\section{Final Monthly Data}

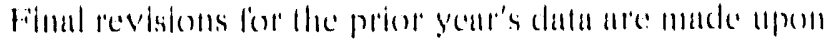

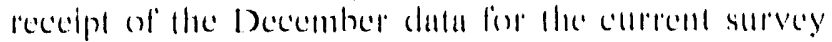
yeall.

\section{Note 10. Natural Gas Policy Act of 1978}

Tables 5 and 8 through 11 , in this publicalion eomlain data reported by the malural gas industry under Tille I of the Naturul Cias Policy Act of 1978 (NGPA).

\section{Title I of the NGPA - Wellhead Prices}

The NGild signed inte law (n) November 9, 1978, mandaled a new franework for the regulation of most lacets of the matural gas industiy. Ceiling prices were established for all production of malural gas. for gas produced firom both new and old (pre-NCil'A) reserves, ceiling prices depend on contract povisions and the characteristics of the well. If a well qualifies under more than one provision in Title l of the NCil'A. the highest maximum ceiling price is applicable, fur. llect, all price ceolings are adjustod by a momthly inllation adjustment.

Natural gas dedicaled to interstate commerce on or hefore the November 9), 1978 enactment of the NGIPA has as a maximum lawful price the "just and reasenable" rate us established by the Federal Energy Regulatory Commission under Section 104.

The maximum lawful price of natural gas under old intrastate contracts (Section 105) is based on its contrace price on November 9, 1978, and is, in gencral, much higher than for comparable old interstate gas. Gas production under Sections 104, 105, and 106 (old contracts that have "rolled over") constitule what is refered to as "old gass."

Almost all development and extension wells begun al (er Fehroary 19, 1977, qualify as new onshore production (Section 10)3). In order to qualify for new gats statlus under Section 102, an onshore well starled alter February 19, 1977, must produce from a new reservoid of be all least 2.5 miles from, of 1 (o) of feet decpere than, " "marker well" (a well producing in commercial cuanfilies any time between Jantury 1, 197(), and April 20, 1977). Offshore gas qualities for Section 102 if production is from new leases (entered into on of after April 2(), 1977) or reservorims "discovered" on of after July 27,1976 .

Production from wells qualifying under Section 107 is

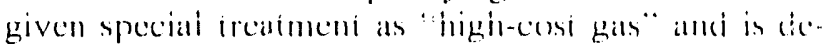
fincel as gas produced:

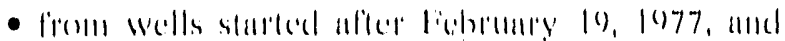

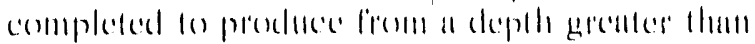
$15,()()()$ licul

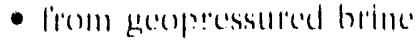

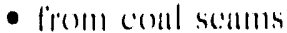

- fromir Deventian shale

- ander comditions delermined by die liceleral linergy Regulatory (ommission to presemll extratordimury risitis or cossts.

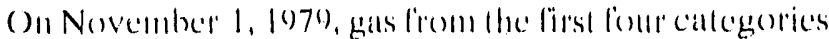
was deregulated. () 1 August 15, 1980, malural gas produced from light formalions (geofogic formation where

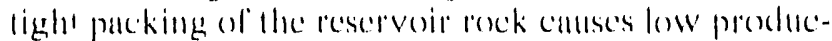
lion ralces) was defined as high-eost gas with a ceciling price sed at $2(0)$ pereent of the Section I0,3 price.

Under the NGI'A, producers applying for price classificution under Secolions 102, 10), 10), or l()8 must like Form liER -121 , with the applicuble Stalc juriselicliomal agency. 'The forms are then sent to litiRC for review. The filings are gleuped aceoreling to the month received by fi:RC: Not all submissions contain estimated atmonal velumes or contract pricess. Ammual voldumes are often estimaled by preducers prion !n actual operations of hased on short periods of operation.

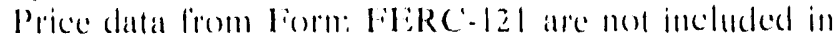
his publicanom, since submission dates vary and prices may have changed subseguent lo stabmission.

() Janlany 1, 1985, lhe following callegories of bas were deregulated pursuant lo NCirA Section 121:

- Section 102(c) "how natural gias"

- Section lo3(c) "new enshore production wells" which were nol comminted or dedicated lo interstate commerec on April 20, 1977, and which prodece gas from a complesion localtion deepere than $5,(x)()$ ficet.

- Secetions 105 and loto(b) "intrastalle gas moler eontmaces" where the price paid on December 31, 1984, is higher than $\$ 1 .(x)$ per million Btu, pros-

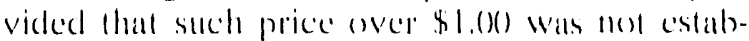
lished by operation of an indefinite cascalantor clause.

()1: July 1, 1987, the following callegory of gits wats cleregulated pursuam an N(i:'A Secoliom.121:

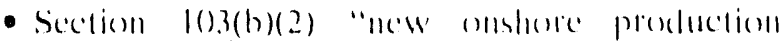

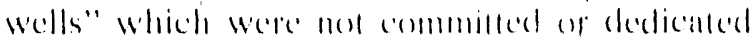
(1) inferstate commoneres (1) April 20, 1977, and

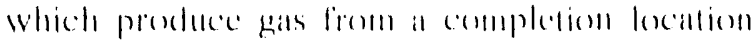

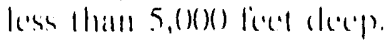

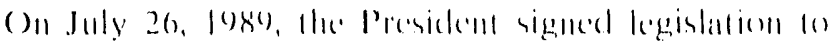

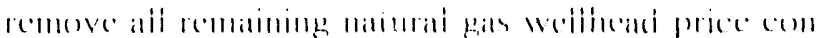

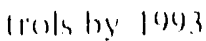


Note 11. Unaccounted For

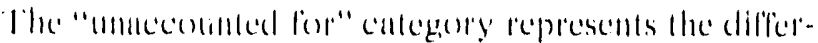

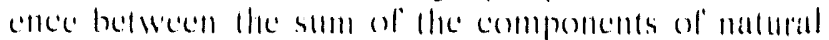
gas supply and the stum of the cemponents a) matural gats dispesition. These differencess may be dac to cuman-

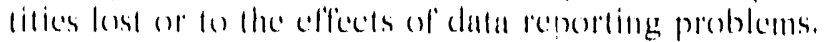
Beporting problems inclade dillerences doe lo the net resula of eomversions of flow dala meleted at varying lemperallates and pressilde hases and converted fo a standard lemperatere and pressure base; the effece of valdiations incompany aceounting and billing practices; differences bedween billing eycles and calendar periols; and imbalancess fesulling firom the mergere of data reporting systems, which vally in sceper, formal, definitions, and lype of respendents.

\section{Annual Data}

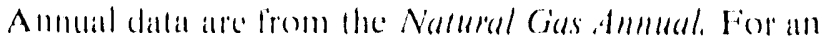
explanation of the methodology involved in calculaling annual "manceomuled for" data, see the Natural Gas .l1111161/1988.

\section{Preliminary Monthly Data}

Preliminary monthly data in the "unaceounted fert" callegory are calculated by subtracting additions 10 storage, exports, and consiumption from total supply/ disposition.

\section{Final Monthly Data}

final momblhly dala in the "unaceounted forp" callegery atre calculaled by subtracting storage additions, exports, and tolat comsumplion from tolal supply.

\section{Note 12. Purchased Gas Adjustments}

The Purchatsed Gas Adjuslment filings for selected in-

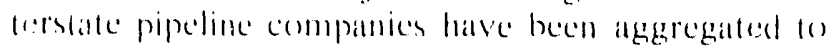
present volume and price dala by Neil'A callegery as shown in Iable 5. These filings represent over 85 percent of the wellhead purchases subject to the N(il'A and dedicaled to the Interstate market.
These filings were submilled every 6 momthes by most

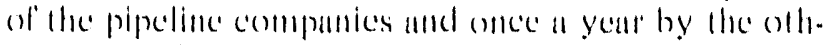
cos. Begimming Junc l, i988, all pipelines will file pro. jections quarterly in an abbrevialed formul, and one detailed lïling pere year. The prefeceled velume and price clata are ased fore each month for which the liting is effective. The filling dales, which are different for lhe different companies, alle shown in Table Al.

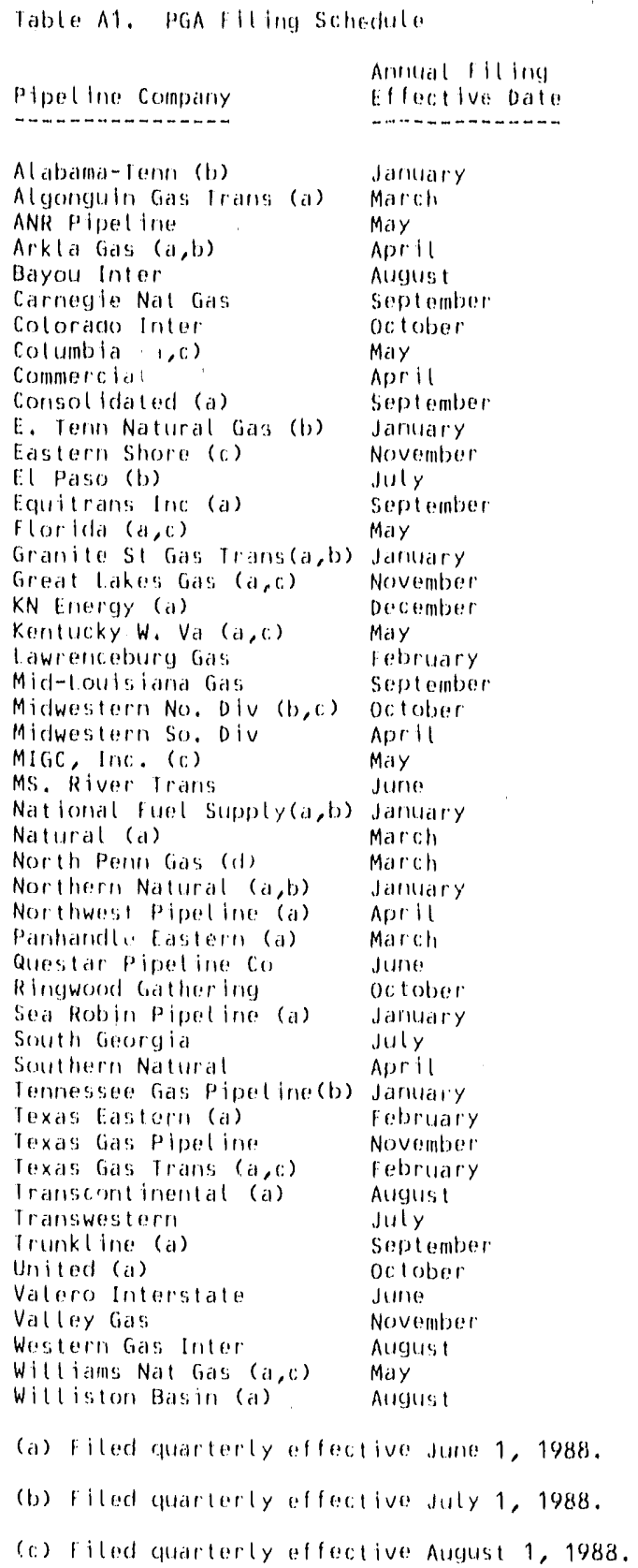


Appendix B

Data Sources 


\section{Appendix B}

\section{Data Sources}

The data in this publication are taken from survey reports authorized by the U.S. Department of Energy (DOE). Energy Information Administration (EIA) and by the Federal Energy Regulatory Commission (FERC). The EIA is the independent statistical and analytical agency within the DOE. The FERC is an independent regulatory commission within the DOE which has jurisdiction primarily in the regulation of electric utilities and the interstate natural gas industry. The ElA conducts and processes some of the surveys authorized by the FERC.

Data are collected from two annual surveys and five monthly surveys. Filings with the FERC also provide sources of data for this publication.

The annual reports are the Form ElA-176, a mandatory survey of all companies that deliver natural gas $10 \mathrm{con}-$ sumers or that transport gas across State lines, and the Form EIA-627, a voluntary survey completed by energy or conservation agencies in the gas-producing States.

The monthly reports include three surveys of the natural gas industry and two surveys of the clectric utility industry. The natural gas industry surveys are the Forms FERC-8 and EIA-19] filed by companies that operate underground storage facilities, the form FERC-11 filed by major interstate natural gas pipeline companies, and the Form EIA-857 filed hy a sample of companies that deliver natural gas to consumers. The electric utility industry survegs are the form EIA-759 filed by all generating electric utilities and the Form FERC-423 filed by fossil fueled plants. Responses to these five monthly surveys are mandatory.

Data in this publication are also taken from tho types of reports that are filed with the FERC under its regulatory functions. One filing is the Form FERC-121. filed by natural gas producers whe are the first seller of gas that qualified for an incentive price. The other filing is the "Purchased Cias Adjustment." which in filed by interstate pipeline companies on allew them th recover changes in their purchase prices fer natural gas abeve the cons of service reflected in their current fiitiizes.
A description of the survey respondents, reporting re quirements, and processing and editing of the data is given on the following pages for each of the surveys. Also show'm are copies of the EIA and FERC survey forms that are sources of data for this publication.

\section{Form EIA-176, "Annual Report of Natural and Supplemental Gas Supply and Disposition"}

\section{Survey Design}

The original version of Form EIA-176 was approved in 1980 with a mandatory response requirement. Prior 10 1980). published data were hased on voluntiry responses 10 Bureat of Mines. U.S. Department of the Interior predecessor Forms BOM-6-134()-A and BOM-6-1.341-A of the same title.

In 1982, the seope of the revised ElA-176 survey was expanded to collect the number of elecertic utility consumers in each State, volumes of gas transported to industrial and electric utility consumers, detailed information on volumes tramsported across State borders by the respondent for others and for the responding company, and detailed information on other disposition. These shanges were incorporated to provide more complete survey information with a minimal change in respondent burden. The 1982 revision of the Form EIA-176 continues of be the basis of the current version of the form. (On March 4. 1985. the Form EIA-176 wath again approved by the office of Management and Budget for use throngh report year 1986.

In 1988. Whe form EIA 176 was revised to include data collection for deliveries of nalurat gat lo commerelal comsumers for the aceount of ofhers. The revised form was approsed for use during report years 1987 thromgh 1989. Response 10 the form comtinues lo be mandatery

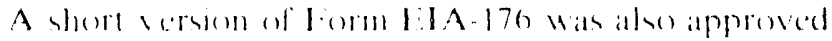

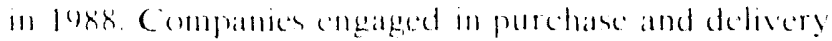

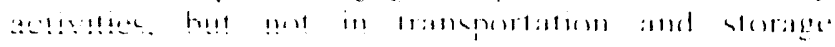


acivivies, may file the shot form. leablly, these ompanies are municipals handling small volumes of gas.

\section{Survey Universe and Response Statistics}

The Form ElA-176 is mailed 10 all identified interstate and intrastate natural gas pipeline companies, investor and municipally owned natural gas distributors, underground natural gas storage operators, synthetic natural gats plant operators, and field, Well. or processing plant operators that deliver natural gas derectly toconsumers (including their own industrial facilities) and/or that transport gas to, across, or from a State horder through ficld or gathering facilities.

Each company and its parent company or subsidiaries were required to tile if they met the survey specifications. The original mailing in February 199() for report year 1989 totaled 2,138 questionnaire packages. To this original mailing. 9 names were added and 2 were deleted as a result of the survey processing. Additions were the result of comparisoms of the mailing list 10 other survey mailing lists. Deletions resulted from post office returm and determinations that companies were out of business, sold, or nol within the scope of the survey Afier all undates. the survey universe was 2.145 responses from approximately 1.800 companices.

Following the original mailing, second request mailing. and nomrespondents followup. 2.145 responses were entered into the data hase. and there were no monrespondents

\section{Summary of Form EIA-176 Data Reporting Requirements}

The EIA-176 is a multiline schedule for reporring all supplies of natural gas and supplemental gaseous fuels and their dispositom within the State indicated. Respondente file completed forms With ElA in Washington. DC. Data for the report year are due by April 1 of the following year. Fxtemsiom of the filing deadline for up to t5 days are granted lo any respendent on reçucut
All natural gats and supplemental gaseous fuels volumes are reported on a physical custody hasis in thousand cubic feet (Mcf), and dollar values are reported to the nearest whole dollatr. All volumes are reported at 14.7 .3 pounds per square inch absolute pressure (psia) and 60 degrees Fahrenheit.

\section{Routine Form EIA-176 Edit Checks}

A series of manual and computerized edit checks are used to screen the Form FIA-176. The edits performed include validity, arithmetic, and analytical checess.

The incoming forms are reviewed prior to keying. This prescan defermines if the respondent identification (ID) number and the company name and add ress are correct, if the data on the form appear complete and reasonable, and if the certifying information is complete.

Manual chects on the data are also made. Sach form is prescanned fo determine that data were reported on the correct lines. The flow of gas through incerstate pipelines is checked at the company level to ensure that each delivery from a State is matched with a corresponding receipt in an adjoining State.

After the data are keyed, computer edit procedures are performed. Edit programs verify the report year. State code, and arithmetic totals. Further tests are made 10 ensure that all necessary data elements are present and that the data are reasonable and internally comsistent. The computerized edil system produces exror listings with messages for each failed edit test. When problems oceur, respondents are comlacted by elephone and required to file amended forms with corrected data.

\section{Other EI.A Publications Referencing Form EIA-176}

Datla from Form l:IA-176 are also published in the Varumal cias dmmual. 
ANNUAL REPORT OF WMIURAL AND SUPPLEMENTAL GAS SUPPLY AND DISPOSITION, $19 \square$

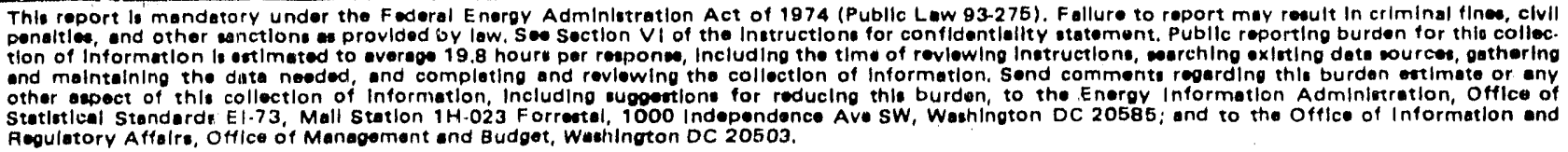

Reguletory Affalrs, OHfice of Management and Budget, Washington DC 20503.

EIA USE

EIA COPY. Tear out, complete, and return to:

Control (ID) No.

Affix mailing label or enter mail address

Energy Information Administration

Mal Station: BG-004 FORSTL

U.S. Department of Energy

Wushington, D.C. 20585

Attr: Form EIA-176

Name: .

Operations in (State):

e Past Office Box: ..........

City, State, Zip Code:

Artention:

PART I: IDENTIFICATION

\begin{tabular}{|l|l|l|l|}
\hline 1.0 Control No. & 2.0 Company Name: & 3.0 Report State & Ela Resubrnittal \\
\hline
\end{tabular}

5.0 Compeny status, name, and/or address change or correction. (Check appropriate box.)

a. Wame and address on mailing label are correct.

b. Change narne, attention line, and/or mail address as indicated below.

c. Company was sold to, or merged with, company entered below.

d. Company went out of business. Customer accounts taken over by company entered below.

e. Other changes, corrections, or comments:

5.1 Change mail addreas to:

o. Company Name:

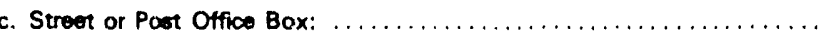

d. City, State, Zip Code:

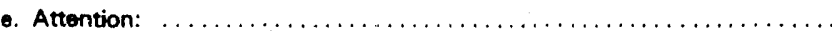

6.0 Contact person:

Name:

Telephone Aros

Number: Code No. No

Ext.

PART II: CERTIFICATION AND DISCLOSURE STATEMENT

1.0 I certity that (Check appropriste box):

a. The information provided herein and appended hereto is true and accurate or, where indicated on the form, reasonable estimetes to the best of $\mathrm{my}$ kirowledge.

b. Dy company does not meet any of the criteria eet forth in Section II, "Who must submit," of the instructions and is therefore not required to complete and submit a Form EIA.176 for the roport State.

c. Does the information supplied on this form contain trade secrets and/or confidential commercial information? $\square$ Yes $\square$ No

\begin{tabular}{|l|c|}
\hline 2.0 Name & 3.0 Titio \\
\hline 4.0 Signature & 5.0 Date
\end{tabular}

Tride 18, USC 1001, makes it a crime for any person knowingly and willingly to make to any agency or department of the United States any false, fictitious o fraudulent statements as 10 any matter within its juriadiction. 
ANNUAL REPORT OF NATURAL AND SUPPLEMENTAL GAS SUPPLY AND DISPOSITION, 19

\begin{tabular}{|c|c|c|c|c|c|c|c|}
\hline \multicolumn{2}{|c|}{1.0 Control No. } & \multicolumn{2}{|l|}{2.0 Company Narne } & \multicolumn{2}{|c|}{ 3.0 Report State } & \multicolumn{2}{|c|}{$\begin{array}{l}4.0 \text { Resubmittal } \\
\text { Date: }\end{array}$} \\
\hline \multicolumn{8}{|c|}{ PART III: TYPE OF COMPANY AND GAS ACTIVITIES OPERATED IN THE REPORT STATE } \\
\hline \multicolumn{3}{|c|}{ 1.0 Type of Company (check one) } & 2.0 & \multicolumn{4}{|c|}{$\begin{array}{l}\text { Gas Activities Operated On-system Within the Report State } \\
\text { (check all that apply) }\end{array}$} \\
\hline & $\ln$ & Investor owned distributor & & a & \multirow{2}{*}{$\begin{array}{l}\text { Produced Natural Gas } \\
\text { Gathered }\end{array}$} & \multirow{3}{*}{$\begin{array}{l}m \\
n\end{array}$} & \multirow{5}{*}{$\begin{array}{l}\text { Delivered for Resale } \\
\text { Delivered directly } \\
\text { to consumers } \\
\text { Other (specify) }\end{array}$} \\
\hline & M & \multirow{2}{*}{$\begin{array}{l}\text { Municipally owned distributor } \\
\text { Interstate pipeline }\end{array}$} & & b & & & \\
\hline & In & & & c & Processed & & \\
\hline & d & Intrastate pipeline & & $d$ & \multicolumn{2}{|l|}{ Purchased } & \\
\hline & S & Storage operator & & e & Transported Interstate & & \\
\hline & s & SNG plant operator & & 1 & \multicolumn{2}{|l|}{ Transported Intrastate } & \\
\hline & $\ln$ & Integrated oil and gas & & 9 & \multicolumn{2}{|l|}{ Stored Underground } & \\
\hline & h & Producer & & h & \multicolumn{2}{|l|}{ Stored LNG } & \\
\hline$i$ & $\mathrm{G}$ & Gatherer & & $i$ & \multicolumn{2}{|l|}{ Injected Propane-air } & \\
\hline i & $\mathrm{Pi}$ & Processor & & i & \multirow{2}{*}{\multicolumn{2}{|c|}{$\begin{array}{l}\text { Produced SNG } \\
\text { Imported }\end{array}$}} & \\
\hline & 0 & \multirow[t]{2}{*}{ Other (specify) } & & $k$ & & & \\
\hline & & & & 1 & Exported & & \\
\hline
\end{tabular}

PART IV: SUPPLY OF NATURAL AND SUPPLEMENTAL GAS RECEIVED WITHIN OR TRANSPORTED INTO REPORT STATE

1.0 Company owned natural gas produced on-system

2.0 Onsystem purchases received

2.1 From producers, gatherers, and/or gas processors

2.2 From pipeline, distribution, and/or storage operators

2.3 From synthetic natural gas plants or SNG pipeline

2.4 At State line or U.S. torder from:

(Company)

(Siate or Country)

(Continue on Part $V I$, if more space is needed)

3.0 Transportation, exchange, and/or storage receipts:

3.1 Received within the report State...

3.2 Received at the State line or U.S. border from:

(Company)

(State or Coun'ry)

(Continue on Part $V I$, if more space is needed)

4.0 Transported into the report State from:

(State or Country)

(Continue on Part VI, if more spece is needed)

5.0 Withdrawn from company operated storage facilities:

5.1 From underground storage

5.2 Fiom liquefied natural gas storage

60 Synthetic natural gas produced

7.0 Other sources of supply (specify):

(Source and/or kind of fuel)

(Continue on Part VI, if more space is needed)

80 Total supply within report Stato

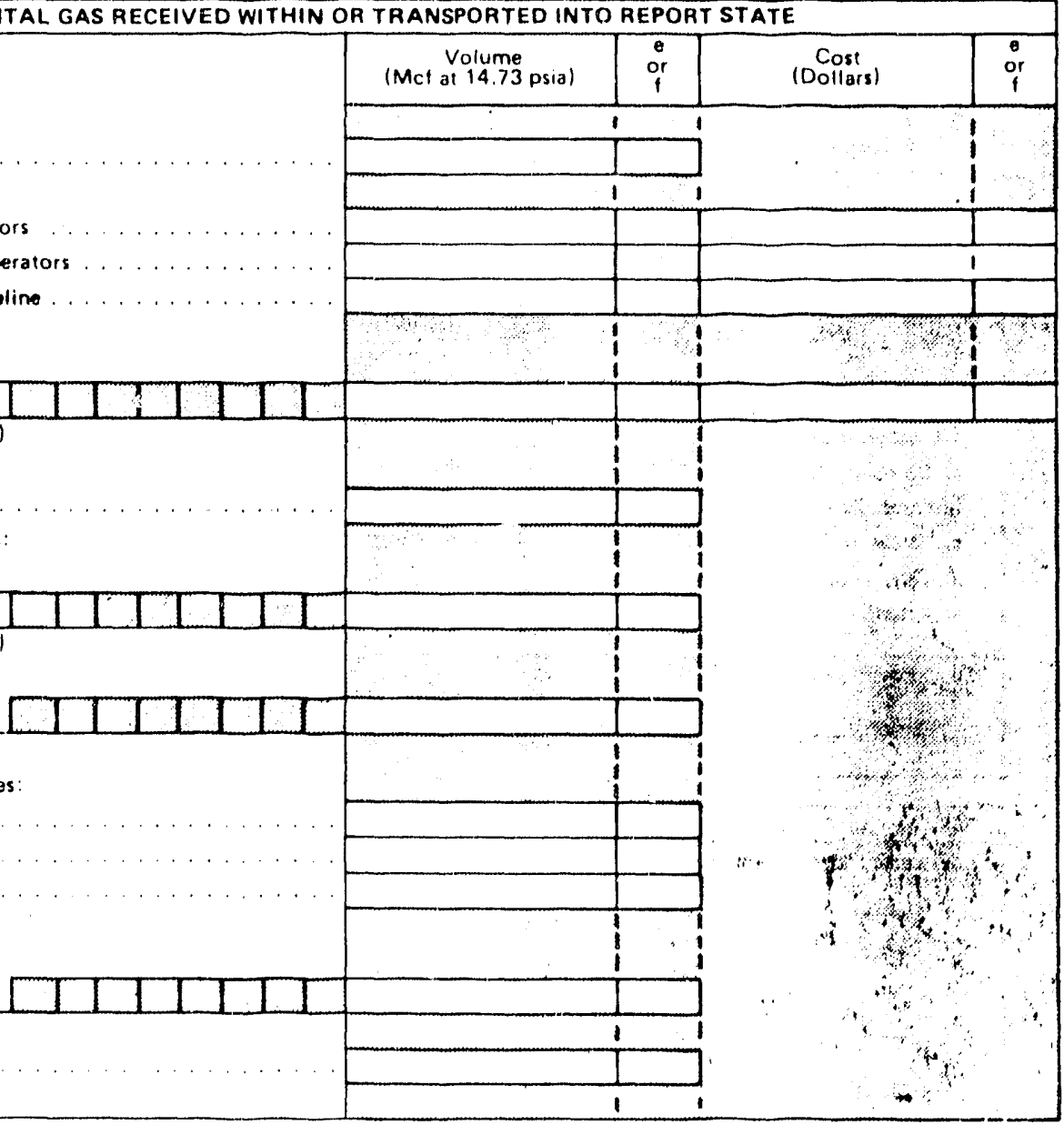


ANNUAL REPORT OF NATUAAL AND SUPPLEMENTAL GAS SUPPLY AND DISPOSITION, $19 \square$

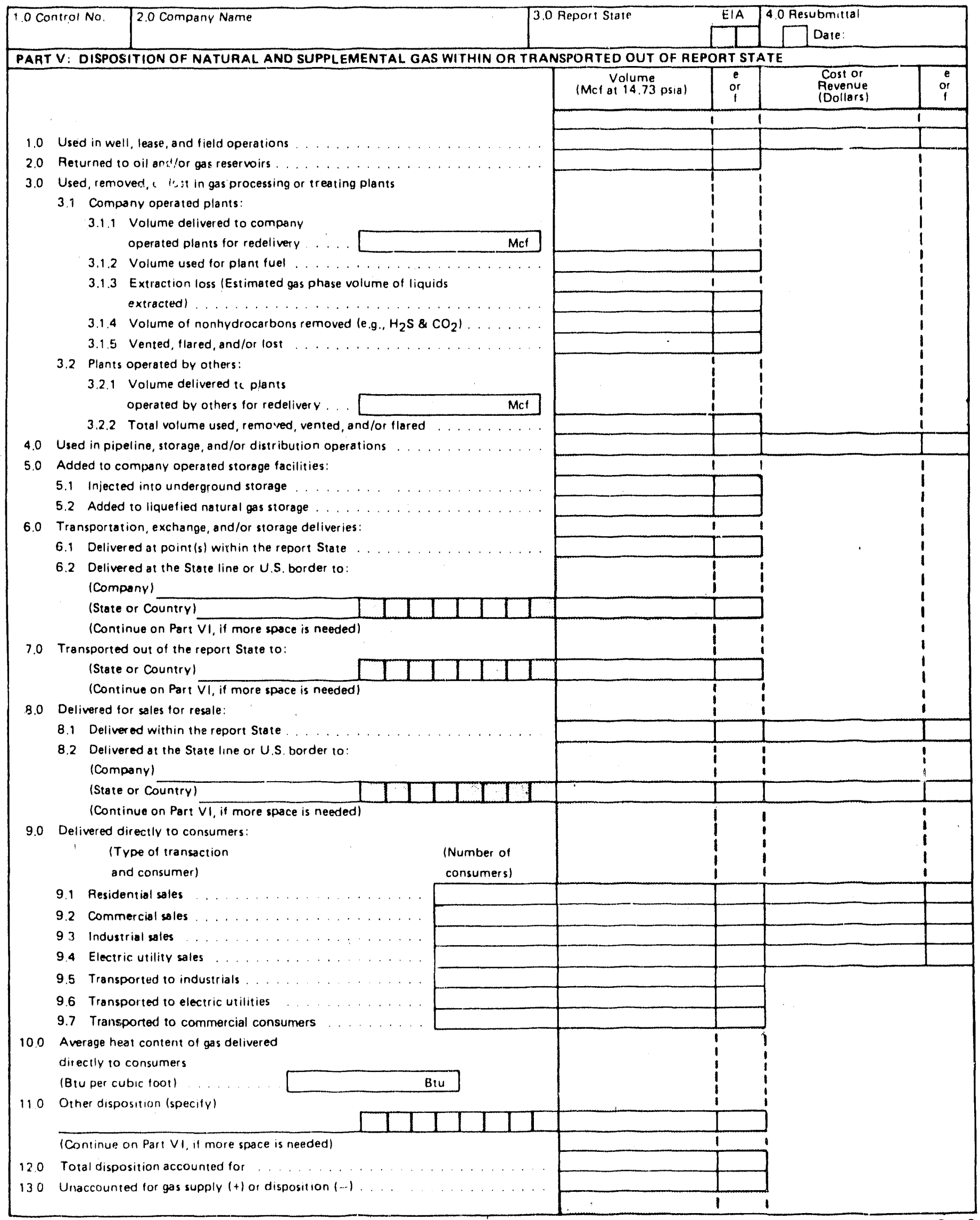




\section{ANNUAL REPORT OF NATURAL AND SUPPLEMENTAL GAS SUPPLY AND DISPOSITION, 19}

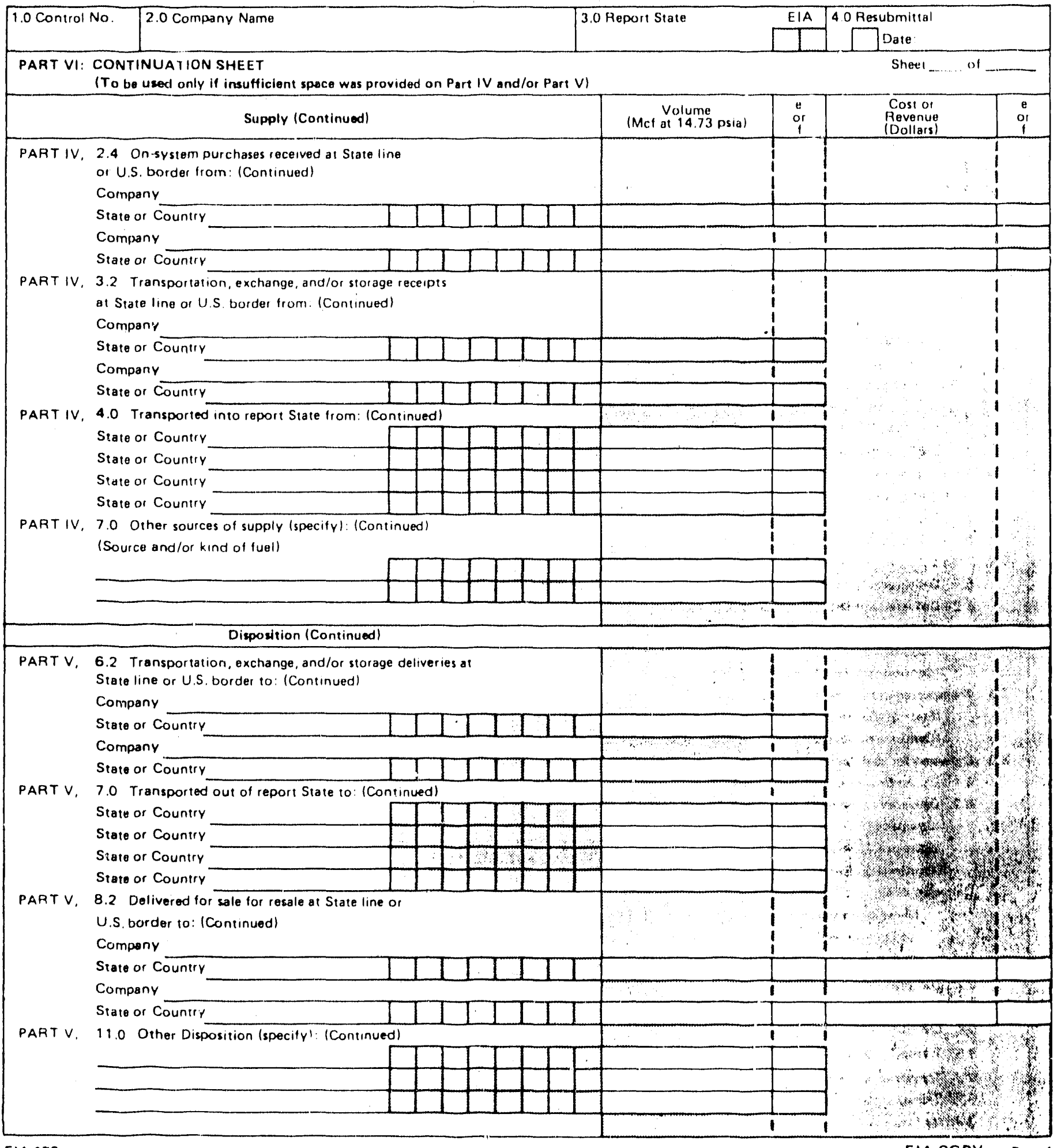


ANNUAL REPORT OF NATURAL AND SUPPLEMENTAL GAS SUPPLY AND DISPOSITION, $19 \square$

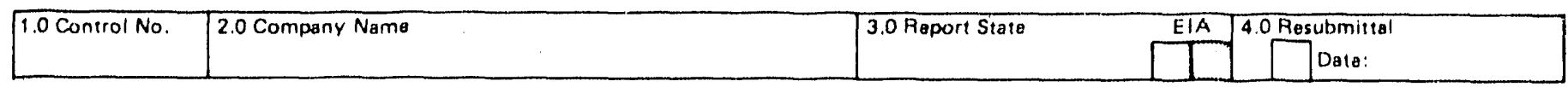

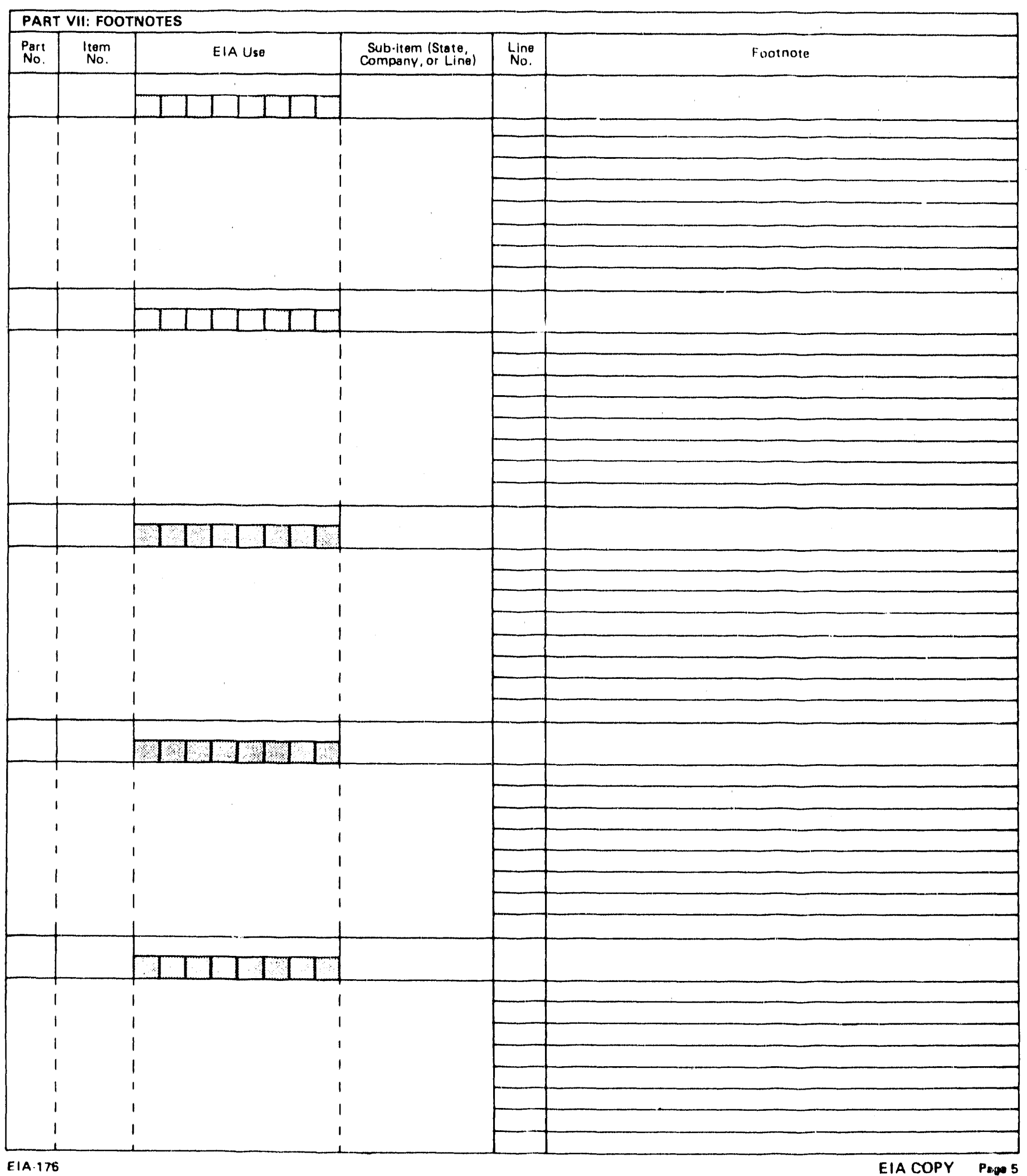




\title{
U.S. DEPARTMENT OF ENERGY \\ ENERGY INFORMATION ADMINISTRATION
}

Form Approved

OMB No. 19060176

Expires: $12.31 / 90$

SHORT

ANNUAL REPORT OF NATURAL AND SUPPLEMENTAL GAS SUPPLY AND DISPOSITION, 19[]$]$ FORM

\begin{abstract}
Thls roport ls mandatory under the Focoral Energy Adminlatratlon Act of 1974 (Public Law 93275 ). Fallure to report may result In criminal fines, clvil penaleles, and other sanctions as provlded by law. See Sectlon VI of the Instructlons for confidentlallty statement. Publlc reporting burden for this colloc. ion of informatlon lo estlmated to average 2 hours per responso, including the time of rovlewing Instructions, searching axlstirg data sources, gatherling other aspect of this collectlon of Information, Including euggestions for reducing thls burden, to the Energy Information Admlalstration, Otfice of Siatstical Standard E1.73 Mall Statlon $1 \mathrm{H} .023$ Forrestal, 1000 Independence Ave SW Washington DC 20585 , and 10 tho Office of Int irmation and Regulatory Affalre, Offlce of Management and Budget, Washlngton DC 20503.
\end{abstract}

EIA USE

A'fix mailing label or enter mail address

Control (ID) No.

EIA COPY. Tear out, complete, and return to:

Erergy Information , Idministration

Mail Station: BG-094 FORSTL

U.S. Department of Energy

Washington, D.C. 20585

Atin: Form EIA-176

Name:

Operations in (State)

Street or Post Office Box:

Citץ, State, Zip Code:

Attention:

A

\begin{tabular}{|l}
\hline PART I: IDENTIFICATION \\
\hline 1.0 Conirol No. 2.0 Company Name: \\
\hline 5.0 Company status, name, and/or address charige or correction. (Check appropriate box.) \\
a. \\
b. Name and address on mailing label are correct. \\
c. Change name, attention line, and/or mail address as indicated below. \\
d. Company was sold to, or merged with, company entered below. \\
e. Company went out of business. Customer accounts taken over by company entered below. \\
Other changes, corrections, or comments:
\end{tabular}

5.1 Change mail address to:

a. Company Name:

b. Operations in (State):

c. Street or Post Office Box:

d. City, State, Zip Code:

e. Attention:

6.0 Contact person:

Name:

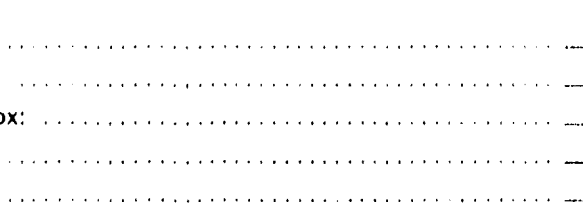


ANNUAL REPORT OF NATURAL AND SUPPLEMENTAL GAS SUPPLY AND DISPOSITION, 19

\begin{tabular}{|l|l|l|l|l|l|}
\hline 1.0 Control No. & 2.0 Company Name: & 3.0 Peport State & ElA & 4.0 Resubmittal & Date: \\
\hline
\end{tabular}

\begin{tabular}{|c|c|c|c|c|c|}
\hline \multicolumn{2}{|c|}{ 1.0 Type of Company (check one) } & \multicolumn{4}{|c|}{$\begin{array}{l}\text { 2.0 Gas Activities Operated On-system W/thin the Report State } \\
\text { (check all that apply) }\end{array}$} \\
\hline a. & \multirow{2}{*}{$\begin{array}{l}\text { Investor owned distributor } \\
\text { Municlpally owned distributor }\end{array}$} & a. & \multirow{3}{*}{$\begin{array}{l}\text { Produced Natural Gas } \\
\text { Gathered } \\
\text { Processed }\end{array}$} & m.I & \multirow{3}{*}{$\begin{array}{l}\text { Dellivered for Resale } \\
\text { Delivered directly } \\
\text { to consumers }\end{array}$} \\
\hline b. & & b. & & n. & \\
\hline c. & Interstate plpeline & c. & & & \\
\hline d. & \multirow{2}{*}{$\begin{array}{l}\text { Intrastate pipeline } \\
\text { Storage operator }\end{array}$} & d. & \multirow{2}{*}{$\begin{array}{l}\text { Purchased } \\
\text { Transported Interstate }\end{array}$} & o. & \multirow[t]{2}{*}{ Other (specify) } \\
\hline e. & & $\boldsymbol{\theta}$. & & & \\
\hline f. & SNG plant operator & f. & Transported Intrastate & & \\
\hline g. & Integrated oil and gas & g. & \multicolumn{2}{|l|}{ Stored Underground } & \\
\hline h. & Producer & h. & \multicolumn{2}{|l|}{ Stored LiNG } & \\
\hline i. & Gatherer & i. & \multicolumn{2}{|l|}{ Injected Propane-air } & \\
\hline i. & \multirow{2}{*}{$\begin{array}{l}\text { Processor } \\
\text { Other (specify) }\end{array}$} & j. & \multicolumn{2}{|l|}{ Produced SNG } & \\
\hline k. & & k. & \multirow{2}{*}{\multicolumn{2}{|c|}{$\begin{array}{l}\text { Imported } \\
\text { Exported }\end{array}$}} & \\
\hline & & 1. & & & \\
\hline
\end{tabular}

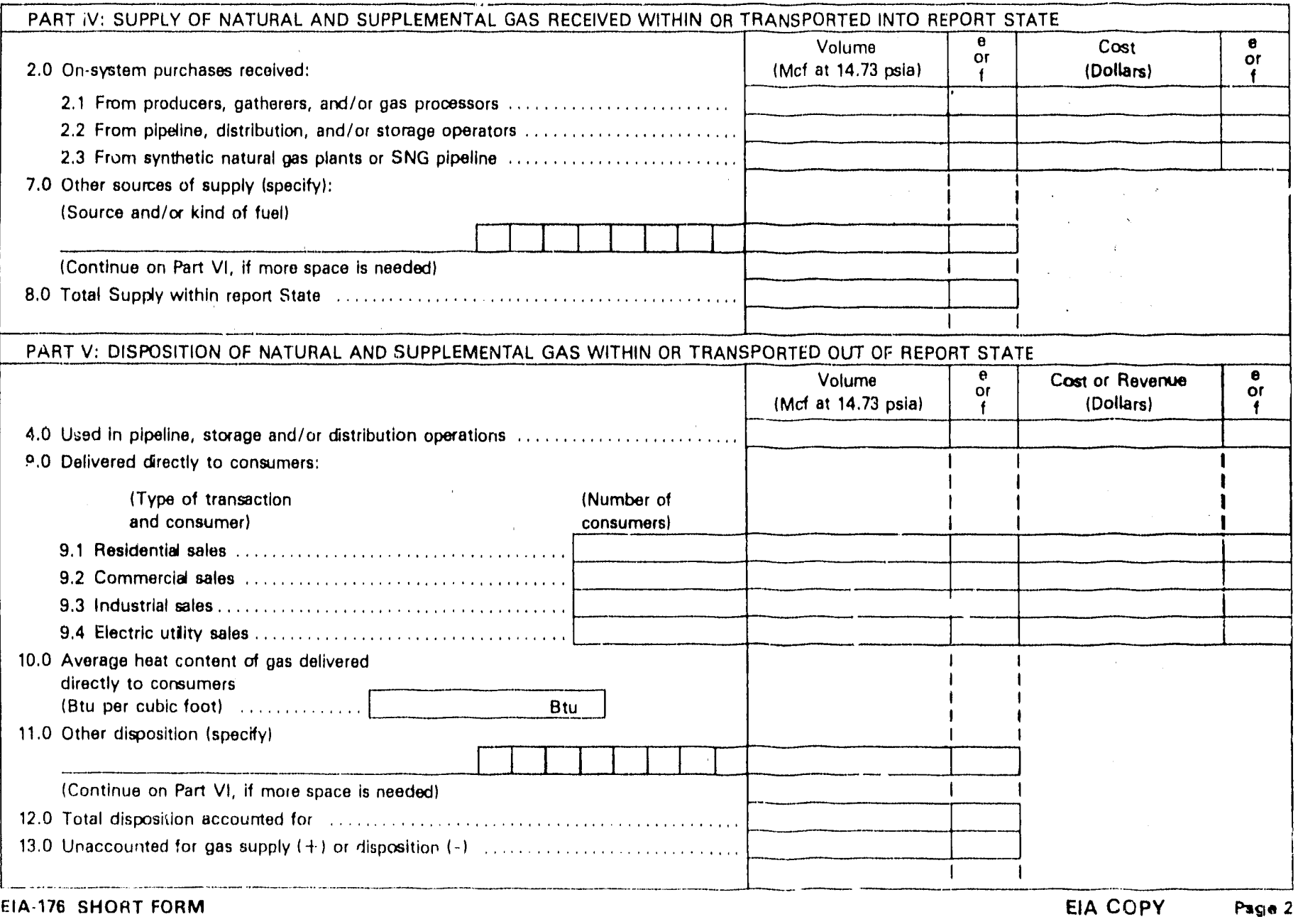


ANNUAL REFORT OF NATURAL AND SUPPLEMENTAL GAS SUPPLY AND DISPOSITION, $19 \square$

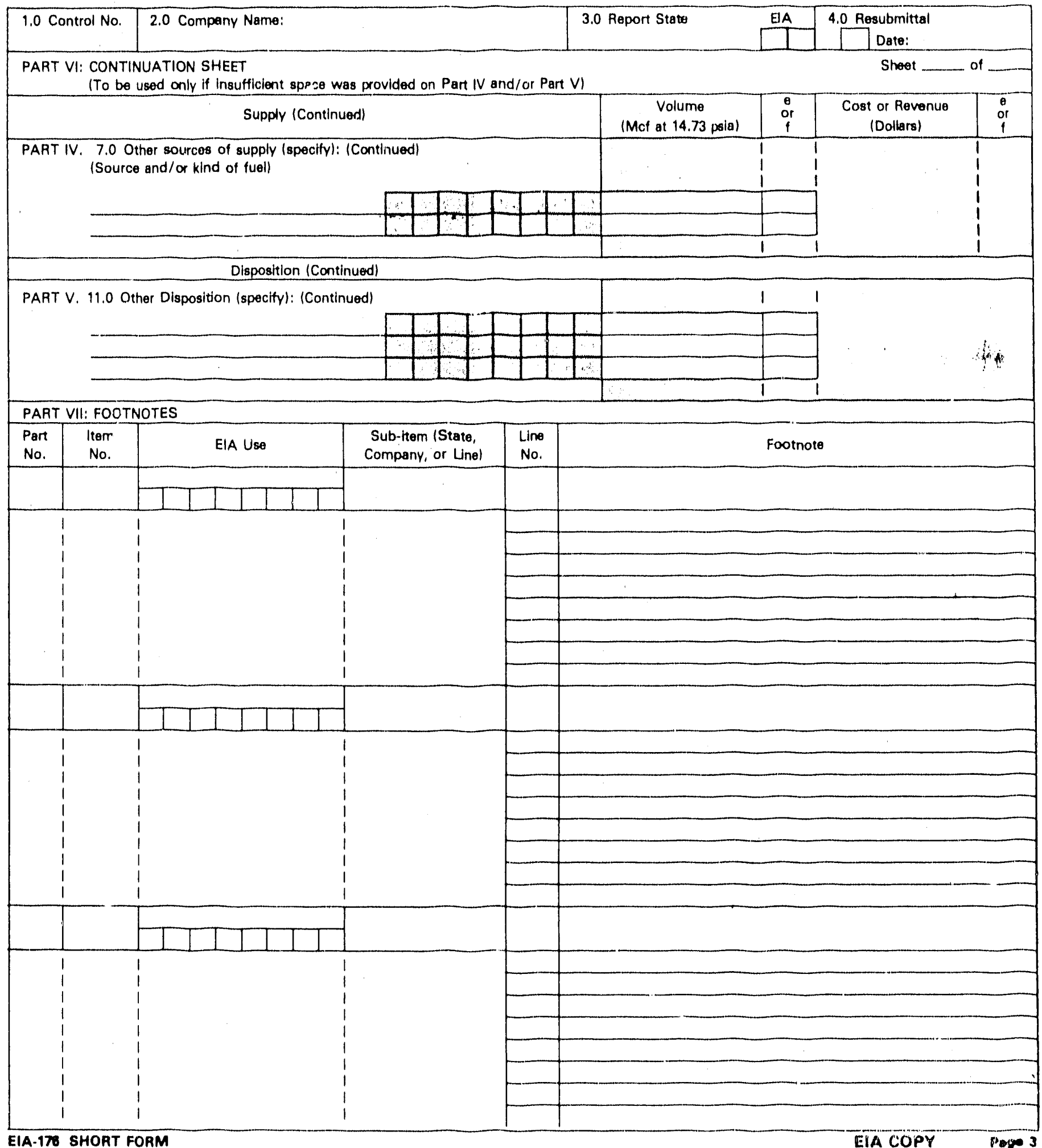




\section{Form EIA-627, "Annual Quantity and Value of Natural Gas Report",}

\section{Survey Design}

Beginning with 198(), matural gas production dala previomsly ohtained on an informall basis from State comservalion agencies were colleceded on liorm Ela-627 (Figure D)2). This form Was designed by LIA to colleed annual matural gats production data from the appropriate State agencies under a standard data reporting system within the limits imposed by the diversity of data collection systems of the various producing States. It was also designed lo averid duplication of effort in eollecting production and value data by producing states and to avoid an monecessary respondent burden on gas and oil well operalors.

\section{Survey Universe and Response Statistics}

Form r1A-627 is mailed to energy or conservation agencies in all 32 nalural gas producing States. All producing States participate voluntarily in the EIA-627 survey by filing the completed form or by responding to telephone contacts. For 1989, data on the quantities of nombydrocarbon gases removed were reported by the appropriale agencies of 22 of the 32 States. These 22 States aceonuted for 57 pereent of total 1989 gross withdrawals. In addition, gross withdrawal data from Kansals, Oklahoma, Louisiana, and Montana, which iogether aceounted for 38 pereent of total production, excluded all or most of the nombydrocarbon gitses removed on leases.

\section{Summary of Form EIA-627 Data Reporting Requircments}

Form EIA-627 is a multipart form that collects data on the production volume of matural gas (including gross withdrawals from both gas and oil wells); vol. umes returned to fomation for repressuring, pessure matinienance, and eycling; quantities vented and flared; quantities of mombedrocarbon gases removed; matrketed production; the value of matketed production; and the number of producing gas wells.

Respondents are asted to report all volumes in million cubic feet at the Stake's standand pressure base and all

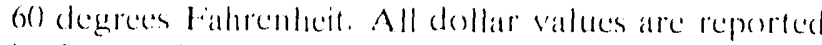
in thousintels

\section{Routine Form EIA-627 Edit Checks}

Each filing of form EIA-627 is manually checked for reasomableness and mathematical accuracy. Informafion on the forme is compared to totals of monthly data reported to the futerstate (O) Compact Commission (sece Appendix B, "Datal Sources"). Volumes are converted, als neecessialy, lo a standard 14.73 psial pressure hase. Reatsomableness of data is assessed hy comparing reported datla to the previous year's data. State ngencies are contacted by telephone to correct errors. Ame nded filings or resubmissions are not a requirement, since participation in the survey is voluntary.

\section{Oiher EIA Publications Referencing Form EIA-627}

Data from form E1A-627 are also published in the EIA publication, Natural Cass dnnual.

\section{Interstate Oil Compact Commission Form "Monthly Report of Natural Gas Production"}

\section{Survey Design}

The Interstate oil Compact Commissiom (IOCC) is an organization comprised of 32 gas and oil producing States; the Governor of each State sits on the board of the IOCC. The IOCC form, "Monthly Report of" Natural Cats Production," (Figure Do3) is a voluntary report filed to the $10 \times C$ " by most of the producing States. The IOCC forwards enpies of these forms fo the EIA. The pupese of the form is to standardize, 10 the extent posible, the reporting of natural gas data by the States.

\section{Survey Universe and Response Statistics}

Most of the 32 Stales report data to the Ioce. Twe exceptions atre filorida, which submils its own form, and Califormat whese data are taken from the Comservation Commillee of (alifornia ()il producess publication. Reports on Statc preduction alle forwarded fo the lis by the Ioce approximately so) daty after the end of the report month. 


\section{ANNUAL QUANTITY AND VALUE OF NATURAL GAS REPORT}

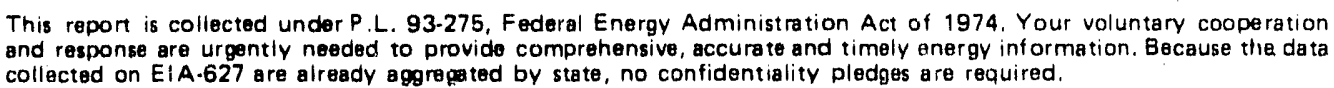

This report is collected under P.L. 93-275, Federal Energy Administration Act of 1974. Your voluntary cooperation and response are urgentiy needed to provide comprehensive, accurate and timely energy information. Because the data collected on EiA.627 are already aggregated by state, no confidentiality pledges are required

\section{PART I: IDENTIFICATION DATA}

\begin{tabular}{l|l|l} 
1. Name of State Reporting & 2. Calendar Year Being Reported
\end{tabular}

\section{Name of Office/Agency}

4. Office Address (Street, City, State, Zip Conde)

5. Name of Contact Person 6. Phoile Number of Contast Person

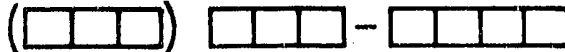

PART II: NATURAL GAS VOLUMES

7. Enter the pressure base at which all volumes are reported (psia at $60^{\circ} \mathrm{F}$ )

8. Gross Withdrawals

\section{REPORT ALL VOLUMES IN MILLIONS OF CUBIC FEET}

Gross withdrawals should represent full well siream volumes including all natural gas plant liquids and nonhydrocarbon gases, but excluding lease condensate. Also, include amounts delivered as royalty payments ar consumed in field operations. PROVIDE BEST ESTIMATE VIHEN GAS IS NOT METERED

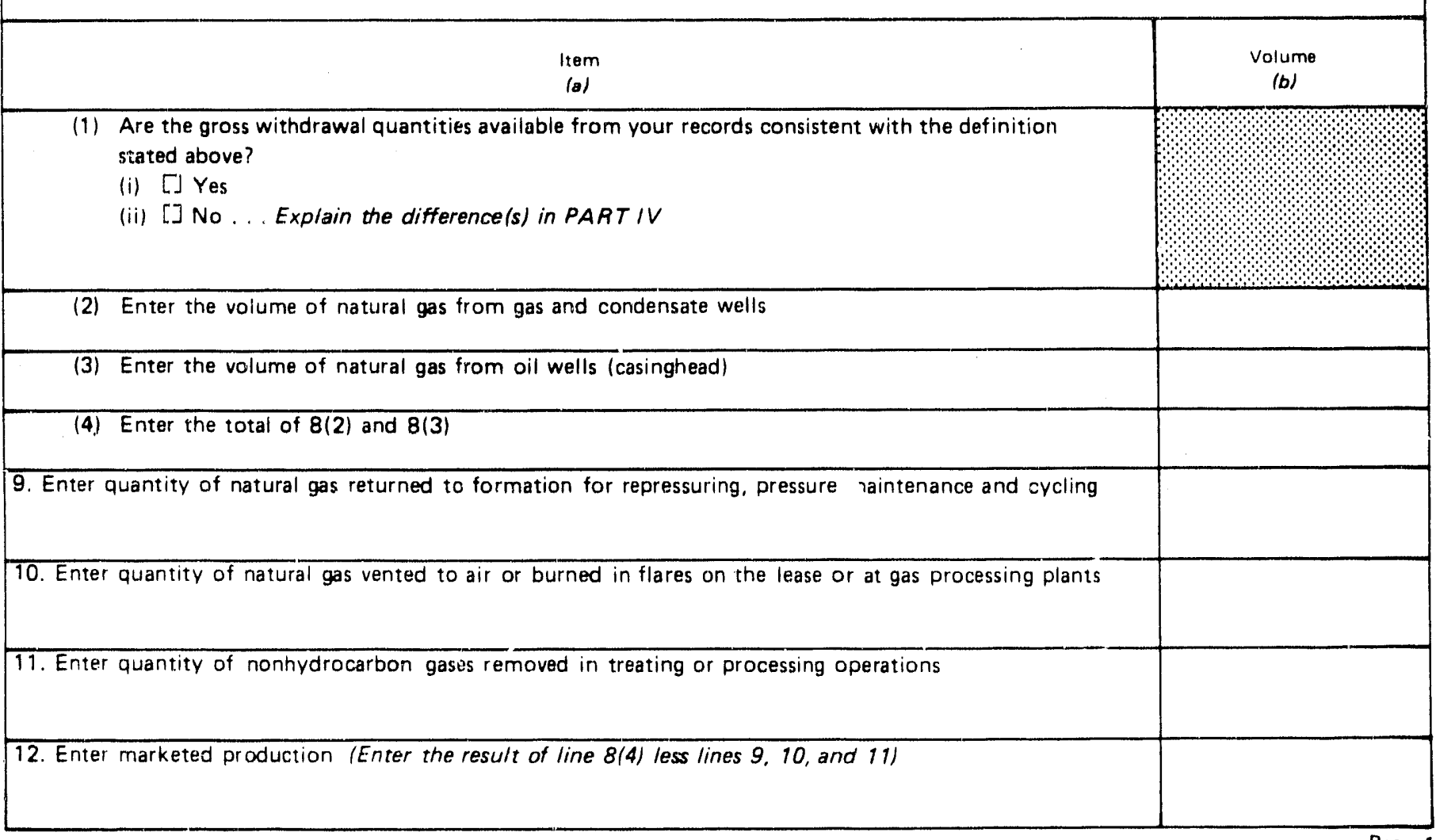




\section{ANNUAL QUANTITY AND VALUE OF NATURAL GAS REPOAT}

PART III: NATURAL GAS VALUES

13. Value of 'Marketed Production (Wellhead Sales Prices)

\section{NOTE}

The value reported on line 14 below should represent wellhead sales prices including charges for natural gas plani liquids subsequent/y removed from the gas and for gathering and compression, in addition to state production, severance, and/or similar taxes.

Complete the table below only if your method of reporting is inconsistent with the above note. For each itern in column (a) of the table, enter an " $X$ " in column (b) or 'c) to indicate whether the item is included or exsluded from the amount reported on line 14 .

\begin{tabular}{|l|l|l|l|l|}
\hline $\begin{array}{l}\text { Line } \\
\text { No. }\end{array}$ & \multicolumn{1}{|c|}{$\begin{array}{c}\text { Item } \\
(a)\end{array}$} & $\begin{array}{c}\text { Excluded } \\
(\mathrm{c})\end{array}$ & \\
\hline (1) & Natural gas plant liquids & & \\
\hline$(2)$ & Lease condensate & & \\
\hline$(3)$ & Gathering and compression charges & & \\
\hline$(4)$ & State production, severance, and/or similar taxes & & \\
\hline
\end{tabular}

14. Enter the available value of marketed production REPORT IN THOUSANDS OF DOLLARS

15. Enter the quantity of marketed production associated with the value entered in 14 . REPORT IN MILLIONS OF CUBIC FEET

16. Enter the total number of producing gas wells in operation as of December 31 for the reporting year.

PART IV: COMMENTS

17. Enter any additional comments you may have, including identification and explanation of any data elements submitted based on definitions differing from those applications to data which you provided for the previous year. If more space is needert, please attach separate sheet(s) and put "sheet of " in the upper right corner of those sheets. 


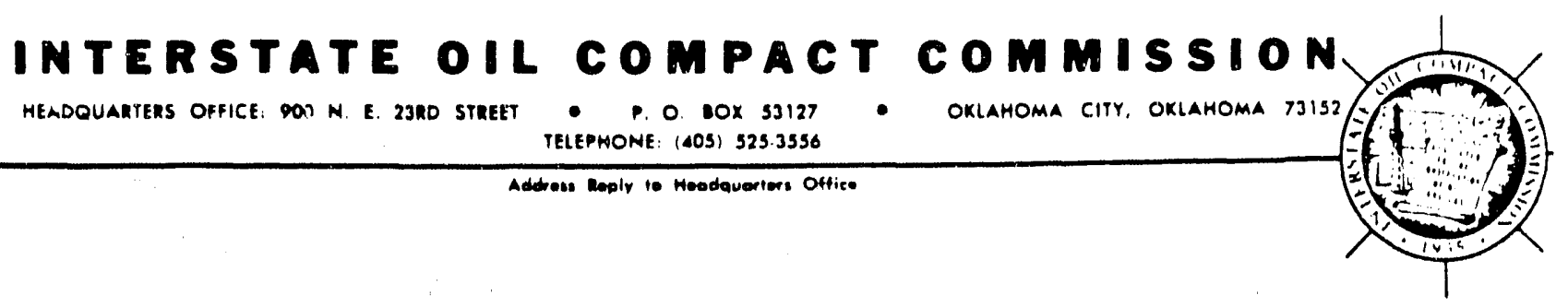

Monthly Report of Natural Gas Production for the State of

Month of

Please return two copies of this report as soon as the figures are available after the close of the month for which data are reported. Reports should be addressed to the Interstate Oil Compact Commission, Box. 53127, Oklahoma City, OK 73152 .

Name of Reporting Agency

Address

Correspondent \& Title

Please adjust all volumes to the same pressure base:

Report pressure base used ( lbs. absolute at $60^{\circ} \mathrm{F}$.)

(Millions of Cubic Feet)

1. Gross gas production*

(a) Natural gas from gas and condensate wells

(b) Natural gas from oil wells (casinghead)

* Include amounts delivered as royalty payments or consumed in field operations. Please provide best estimates when gas is not metered.

2. Quantity of natural gas returned to formation for repressuring, pressure maintenance and cycling.

3. Quantity of ratural gas vented to air or burned in flares on the lease or at gas processing plants.

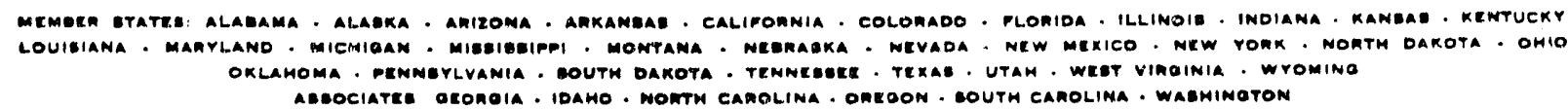




\section{Summary of Data Requirements}

The ace form comsists of the questions an one page, and requires folumetric information on gross production, quantitices of gars venfed or flared, and gas used for repressuring.

\section{Routine Edit Checks}

State data are checked for reasonahleness and, in the cvent of problems, the appropriate state agency is called.

\section{FERC-8/EIA-191 Surveys, "Underground Natural Gas Storage Report"}

\section{Survey Design}

This survey was jointly implemented in 1975 by the Federal Power Commission (FPC), the Federal En(rgy Administration (FEA), and the Bureau of Minces (BCM) as the FPC-8/ FEA-C-318 system. The data recoved on hoth the FPC-8 and FEA-G-318 were computerized and aggregated by FPC.

At the beginning of 1979 , the ElA assumed responsibility for the collection, processing, and publication of the data gathered in the survey. Form FEA-G-318 was renewed on July 1, 1979, as Form EIA-191 and the survey was retilled the FPC-8/FIA-19) Survey (Figure D4 show's the EIA-191). Form FPC-s wats renewed in December 198.5 and the survey relitled FERC-8/ EIA-19l Survey. The forms have not been merged because of HERC's stated desire to maintain the separate identity of the HERC-8 for administrative reasons.

\section{Survey Universe and Response Statistics}

Currently, 91 companies operate underground storage facilitices (of these companies, 4 () are subject to the jurisdiction of FERC and are required foreport data on Form FERC $-x$. The other 51 companies are required to file the essentially identical Form ElA-191. Both forms are filed with EIA and the data are merged in a unified data processing yystem.

The response rate as of the filing deadline is approximatcly 20 percent. Data from the remaining 80 percem sf respondents are received in writing and/or by ketephome within 3 for tays after the foling deadline. All data supplicet by telepheme are subsequently filed in writing. generally within 15 days of the filing deadline. The final response rate is 10() perecent.

\section{Summary of FERC-8/EIA-191 Data Reporting Requirements}

The virtually identical forms FERC -8 and EIA-191 are mulipart forms that report the quantitices of gas in storage, injections and withdrawals, and the location (including State and county), and capacity of underground storage reservoirs. Information on co-(owners of slorage reserverirs is also required.

Collection of the survey is on a custody basis, although some respondent ownership data are required. Information requested must be provided within 10 days af ler the first day of each month. Twelve reports are required per calendar year. Respondents are required to indicate whether the data reported are actual or estimated. In the case of most estimated filings, the necessary revisions are reflecked in subseyuent sched: led filings or in a revised submission that provides aco: al data filed, although there is no specific requirement that the respondent do either. Actual data on natural gas injections and withdrawals from underground storage are based on metered quantities. Data on quantities of gas in storage and on storage capacity represent, in part, reservoir enginecring evaluations. All volumes are reported at 14.73 psia and 60 degrees Fahrenheit.

\section{Routine Forms FERC-8/EIA-191 Edit Checks}

Data received on Form HERC-R are merged with data received on Form EIA-19) in a unified data processing system. The survey's five principal data elements (total, base, and working gas in storage, injections, and withdrawals) are hand labulated to provide preliminary totals.

Individual company reports are mamually checked for reasonableness by comparing current reports with prior responses. Mathematical calculations are manually chected for accuracy and all data are checked for internal comsintency. Respondents are required to refile reports combaning any inconsistencies or corrors.

\section{Other EIA Publications Referellcing Forms FERC-8/EI.4-191}

The LIA publication Gonthly Emerge Revien contains data from the FER(-h/LIA-19) survey 


\section{NDERGROLND GAS STORAGE REPORT}

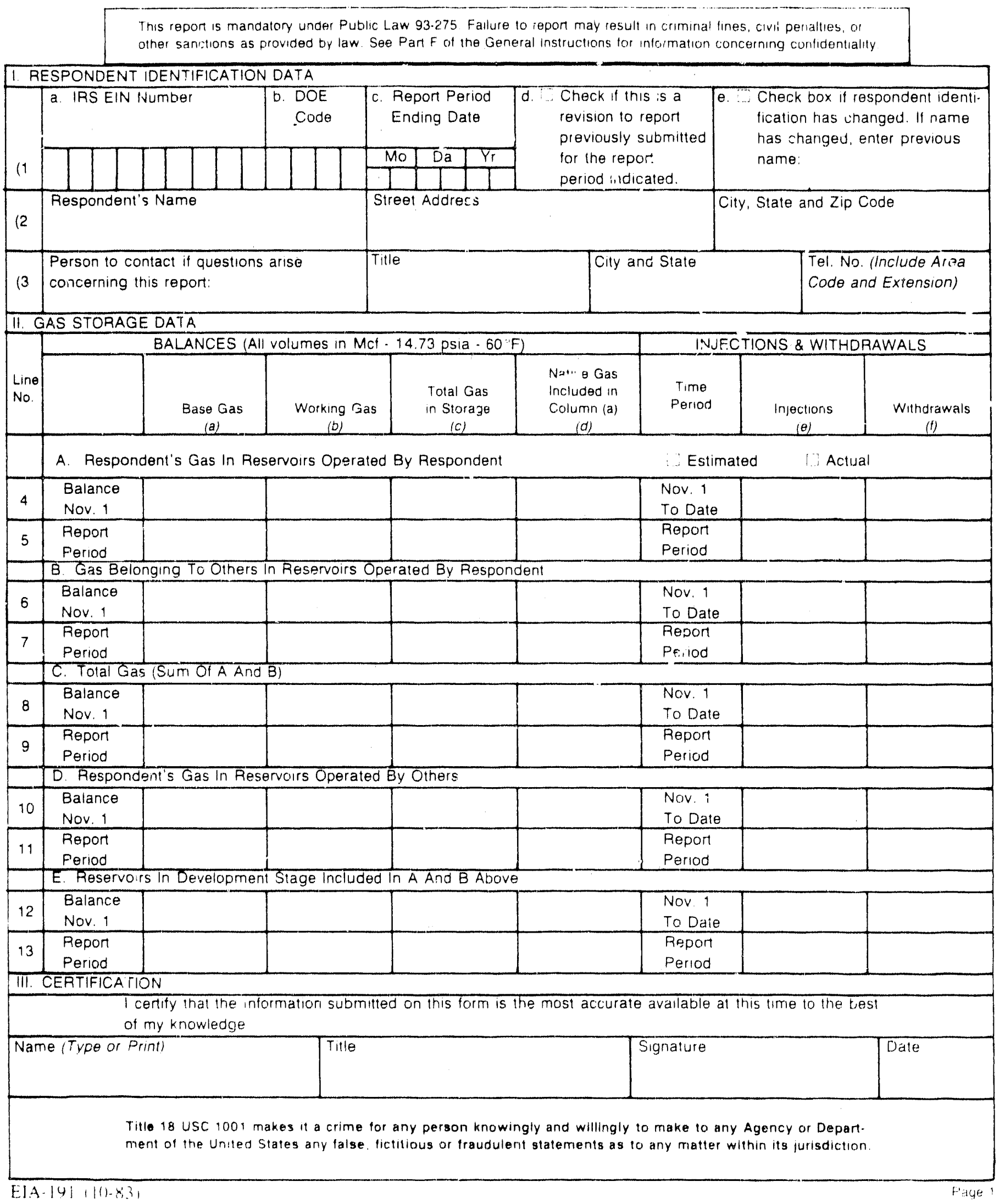




\section{Form FERC-11, "Natural Gas Pipeline Company Monthly Statement"}

\section{Survey Design}

The collection of monthly data from major pipeline companies was begun in December 1904 hy the Federal Power Commission (FPC). On October 1, 1977, FPC ceased to exist, and its functions and regulatory responsibilities were transferred to the Secretary of Energy and to the lederal Encrgy Regulatory Commission (IERC), an independent commission within the Department of Energy.

Information collected on Form FERC-11 (Figure D5) is used by FERC in carrying out its regulatory authority. Form FERC-1] is a momthly regulatory reporting form rather than one filed for statistical purposes.

\section{Survey Universe and Response Statistics}

Form FERC -11 is filed by major interstate natural gas pipeline eompanies whose combined sales for resale and gas transported interstate or stored for a fee exceeded 50 hillion cubic feet in the previous calendar year. Approximately 40 pipeline companies report data on Form FERC-11. Natural gas nipelinc companics are monitored annually fo determine whether each has met the requirements for classification as a major pipeline.

Information is collected monthly hy mail. Historically, the response ralle has been 100 pereent.

\section{Summary of Form FERC-11 Data Requirements}

form F-EKC-11 requires infomation on revenues, expenses, and sales data, as well as volumelric dala (m) purchases and production.

Submission of form FlikC -11 is required no later than 40 days after the colese of the report month. The form requires reporting of hesta preliminary data for the report month and final data fore the same momb in the previous gear. All data are reported ont an equity basis.

\section{Routine Form FERC-:11 Edit Checks}

Ditlat are collected on standard forms that initially are mantatly reviewed and coded. Reviews are made to consure consistency in reporting withen and among utilities in the presentation of current and 12-month financial and sales data. Also, receipts and disposition of gals are analyed between domestice and foreign producers. Data are later sent for keying. which begins the antermated processing. Datla are keved to tape or disk for data editing. Edit reports are produced and are reviened mambially.

\section{Other EIA Publications Referencing Form FERC-11}

The Energy Information Administration publicalion Momthly Eimerge Review contains data from form HERC-11.

\section{FERC-121, "Application for Determination of the Maximum Lawful Price Under the Natural Gas Policy Act"}

\section{Survey Design}

Form FlaRe-121 (Jigure D6) was designed by the Federal Encrgy Regulatory Commission (FERC) to carry out its authority fo regulate natural gats pricess under the Natural Gas Policy Act. Form FERC-121 is intially filed with the agency having jurisdiction over the lease on the land where the well is drilled, for example. State agencies of the Department of Interior. The agencies determine whether or mot to grant the application. The application, support documentation, and decision are forwated fo FRRC, which hats 4.5 days lo review the decision.

\section{Survey Universe and Response Statistics}

form Fi:RC - 121 muse be filed by any natural gas prodecer whos is the first seller of gas that qualifices for an

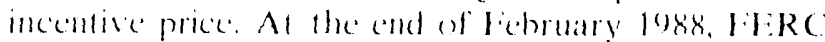

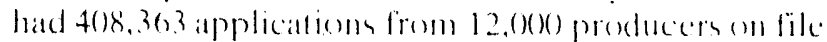
(of the total applications, approsimately ze pereent atre reapplicatione requestinge mew price determintations on

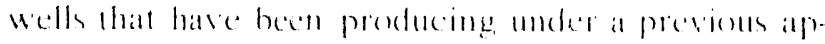
plication. 
Form Approved, OMB No. 1902-0032

U.S. DEPARTMENT OF ENERGY
Federal EnergY Regulatory COMmission

Energy Regulatory Comm
Washington, D.C. 20426

FERC FORM NO. 11: NATURAL GAS PIPELINE COMPANY MONTHLY STATEMENT

This report is mandatory under the authority granted by Sections 10 and 16 and sanctions provided by Section 2 flbl of the Natural Gas Act.

(Expires 6-30-90)

I: IDENTIFICATION

1. Name of Company

2. Address of Cumpany

(1) Number and Street

(2) City

(3) State

(4) Z॥ Code

3. Person Authorized To Sign This Report

(1) Signature

(2) Phone Number (Including area code)

\begin{tabular}{|c|c|c|c|c|c|c|}
\hline \multirow{2}{*}{$\begin{array}{c}\text { Line } \\
\text { No. } \\
\text { (a) }\end{array}$} & \multirow{2}{*}{$\begin{array}{l}\text { Item } \\
\text { (b) }\end{array}$} & \multicolumn{2}{|c|}{$\begin{array}{l}\text { Revenues } \\
\text { ('n thousands of dollars) }\end{array}$} & \multicolumn{2}{|c|}{$\begin{array}{c}\text { Sales } \\
\text { (In millions of cubic leet) }\end{array}$} & \multirow{2}{*}{$\begin{array}{c}\text { Foot- } \\
\text { note } \\
(g)\end{array}$} \\
\hline & & $\begin{array}{l}\text { Current Year } \\
\text { (c) }\end{array}$ & $\begin{array}{l}\text { Previous Year } \\
\text { (d) }\end{array}$ & $\begin{array}{c}\text { Current Yoar } \\
\text { (e) }\end{array}$ & $\begin{array}{c}\text { Previous Year } \\
\text { (f) }\end{array}$ & \\
\hline 4 & Sales of Natural Gas & & & & & \\
\hline 5 & Firm Industrial & & & & & \\
\hline 6 & Off..peak Industrial & & & & & \\
\hline 7 & Interruptible Industrial & & & & & \\
\hline 8 & $\begin{array}{l}\text { TOTAL INDUSTRIAL } \\
\text { (Enter totel of lines } 5,6 \text { and } 7 \text { ) }\end{array}$ & & & & & \\
\hline 9 & Other Ul:ımate Consumers & & & & & \\
\hline 10 & $\begin{array}{l}\text { TOTAL SALES TO ULTIMATE CONSUMERS } \\
\text { (Enter total of lines } 8 \text { and } 9 \text { ) }\end{array}$ & & & & & \\
\hline & Sales For Resale (483) & & & & & \\
\hline 11 & Total Major Gas Pipelines & & & & & \\
\hline 12 & Total to All Other Pipelines and Gas Utilities & & & & & \\
\hline 13 & $\begin{array}{l}\text { TOTAL SALES FOR RESALE } \\
\text { (Enter total of lines } 11 \text { and } 12 \text { ) }\end{array}$ & & & & & \\
\hline 14 & $\begin{array}{l}\text { TOTAL SALES OF NATURAL GAS } \\
\text { (Enter total of lines } 10 \text { and } 13 \text { ) }\end{array}$ & & & & & \\
\hline 15 & Intracompany Transters (485) & & & & & \\
\hline 16 & Revenues From Transportation of Gas of Others (489) & & & (Enter volume & is transported: & \\
\hline 17 & Provision for Rate Refunds (496) & & & & & \\
\hline
\end{tabular}

FERC FORM NO. 11 (REVISED 12.84)

Page 1 of 4 


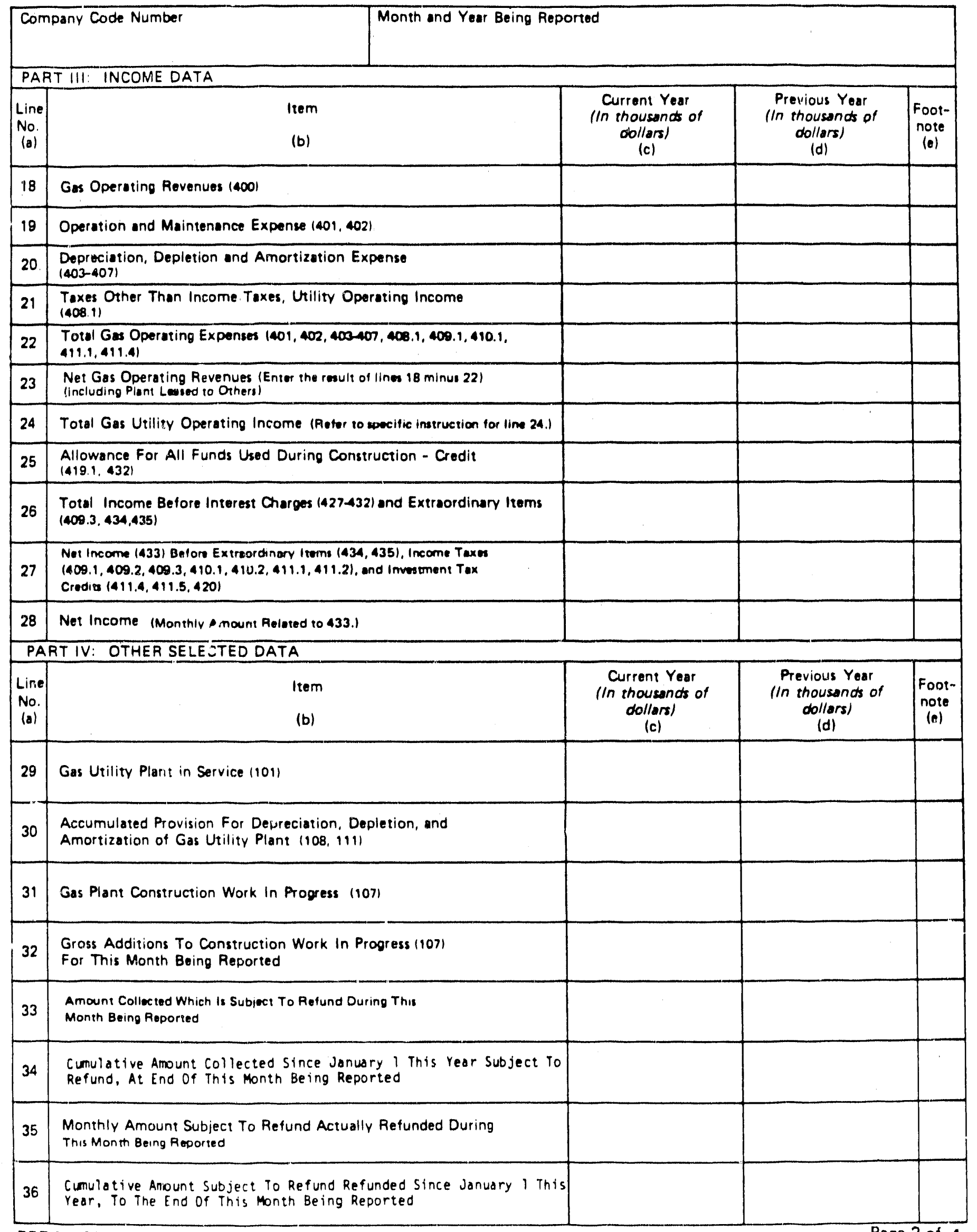

FERC FORM NO. 11 (REVISED 12.84)

Page 2 of 4 


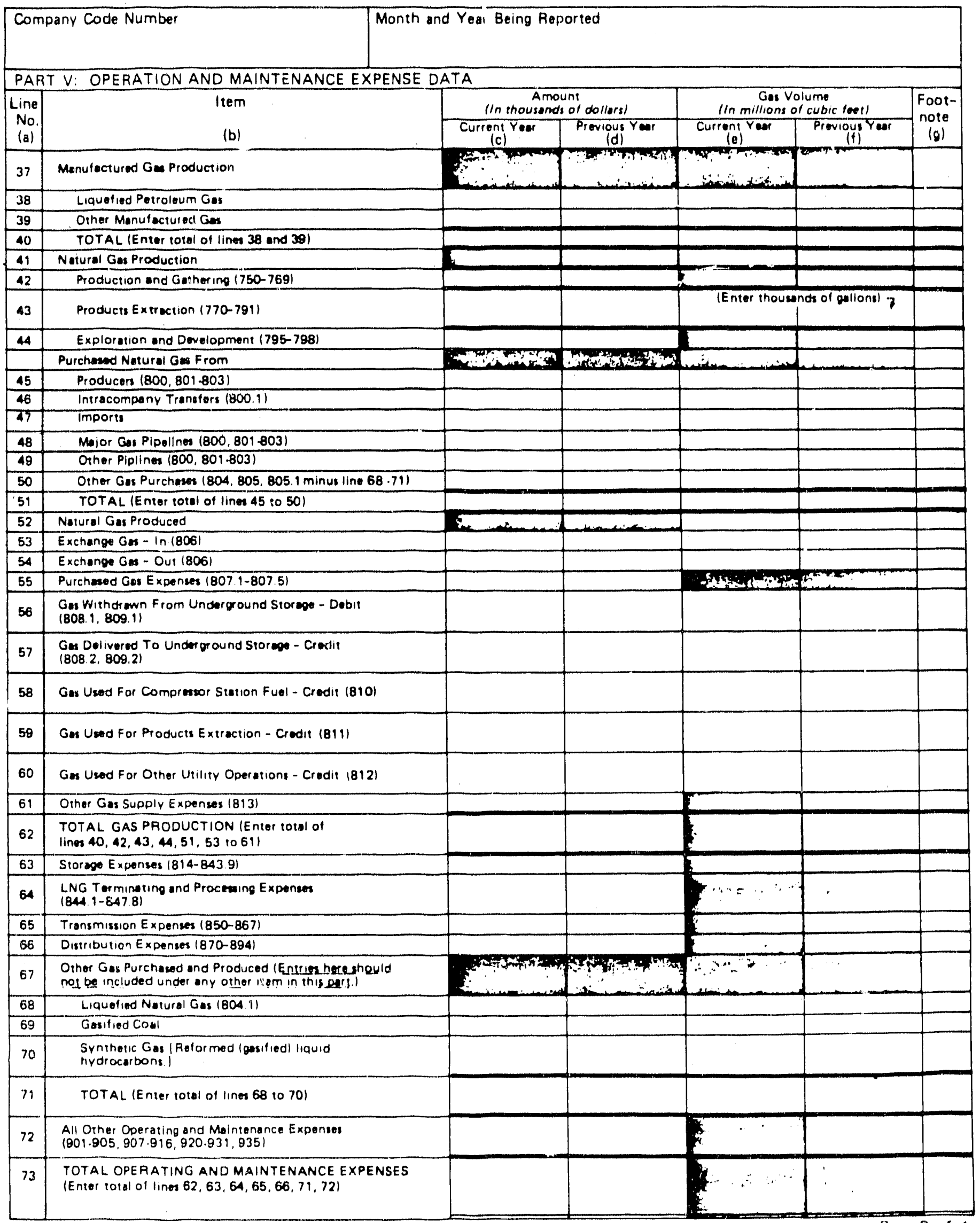




\section{Summary of Form FERC-121 Data Reporting Requirements}

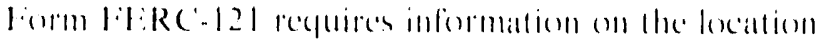
and A'l number of the well, fle mame and address of the applicant, the lype af delemination being sollght.

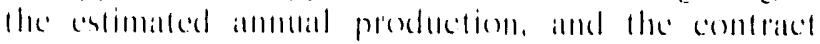
pricis.

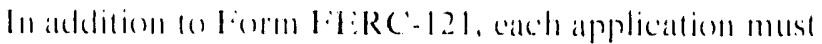
be submilled with smpport docementation; lore examples, a well compledion repont for Section lo.3 fïlings, and

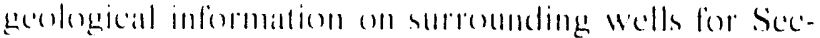
lion lor applicillions.

\section{Routine Form FERC-121 Edit Checks}

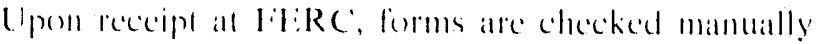

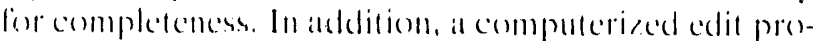
gram Mage incomplete or incorrect data.

\section{Purchased Gas Adjustment Filings}

The purpose of the Pe is is to allow inferstate natural gas pipeline companies a lo recover changes in the pur purchase pricen for matural gas above the cesst of service reflected in lheir courent tarift filings. To allow recov". cry in a timely manner, the key data reported in the Peis are projections of purchatses in the immediate future. In most calses, companies report both the NGPA Section number eovering each combacted purchase and the date of the combrat. These data mate the Pe iA filings a unique sourece of information about the corrent stalce of the natural gats matkel.

\section{Description of Data}

Fifiy-eight interstate pipeline companics submit P' $\mathrm{BA}$ filings. Pipelins: compantes that traispont only intratstate supplices of natural gats do noul submit filings, nof do compantes that have no purchased gas adjustment clatuses in their tarifis.

Prior 10 July 1, 1984, filings for 20 (1) the latgest interstate pipeline companies were included in Table 5. Together, they alceosulted for between 86 and 88 pereent of all interstalce wellhead purchatses reported on liom

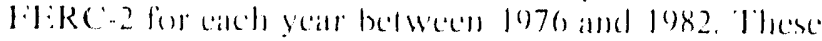

(empanies were also the subjece of an informal 1970)

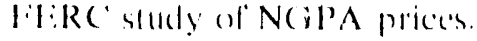

The portion ol the Pe is filing used wats generally re-

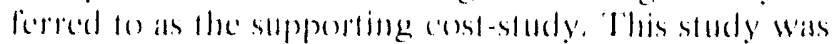
und filed in a speceifice formal or for specilied time rieds. As a result, the lesel of detail included varied from company focompany, ats dicl the exalch lime periond covered. In most casces, howevere, compantes filed price and volume data for llocir purchatse contracts, alomg with the lollowing:

- Contrial dilte

- Noil'a calcegory

- (icosgraphic somico

- firRc account callegory.

For the 20 pipeline compantes, the timing of l' i A submissions varied in two ways:

- Seventeder companies reported dala semijanmually, white threce reported anmually.

- The dater on which the filings hecante effertive and the periods covered by the filings varied.

Because of the lack of a specified form for PCi A filings, calch submission was processed scpalratcly. Major adjustments required to transform the I arious submissions into al single dala base are described in the secelion on datia transformations.

Effective July 1, 1984, Form 542-Pe is required hall certain purchatsed gas cost information that is commonom (o) all P' is lilings be sed forth in a standardized formall. fiblings received alter July 1, 1984, are now being included in rable 5 as they are eompulerized and hecome available. Eventually all pipeline companies hat file the lorm 5.42-l's is will be included in the lable. It is expected that there will be appreximallely 54 cempanies includecl.

All PCiA filinges included projected purchases for a delined future period. Projected volumes and prices represent the company's best estimalce of natural gas purchase volumes and costs during the subsequent effective tariff period. The figures are usually derived

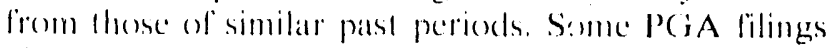
also include "actual" volumes and prices for some past

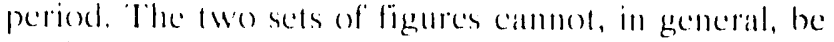
used to check cach ofher hecause the actual ligures call cover periods ofler hath past projections. Fo the extemt hath the twe sels of ligures cant be compared, lley appear (o approximate bul mol fo malch cach other. 


\section{APPLICATION FOR DETERMINATION OF THE MAXIMUM LAWFUL PRICE UNDER THE NATURAL GAS POLICY ACT (NGPA)}

(Sections 102, 103, 107 and 108)

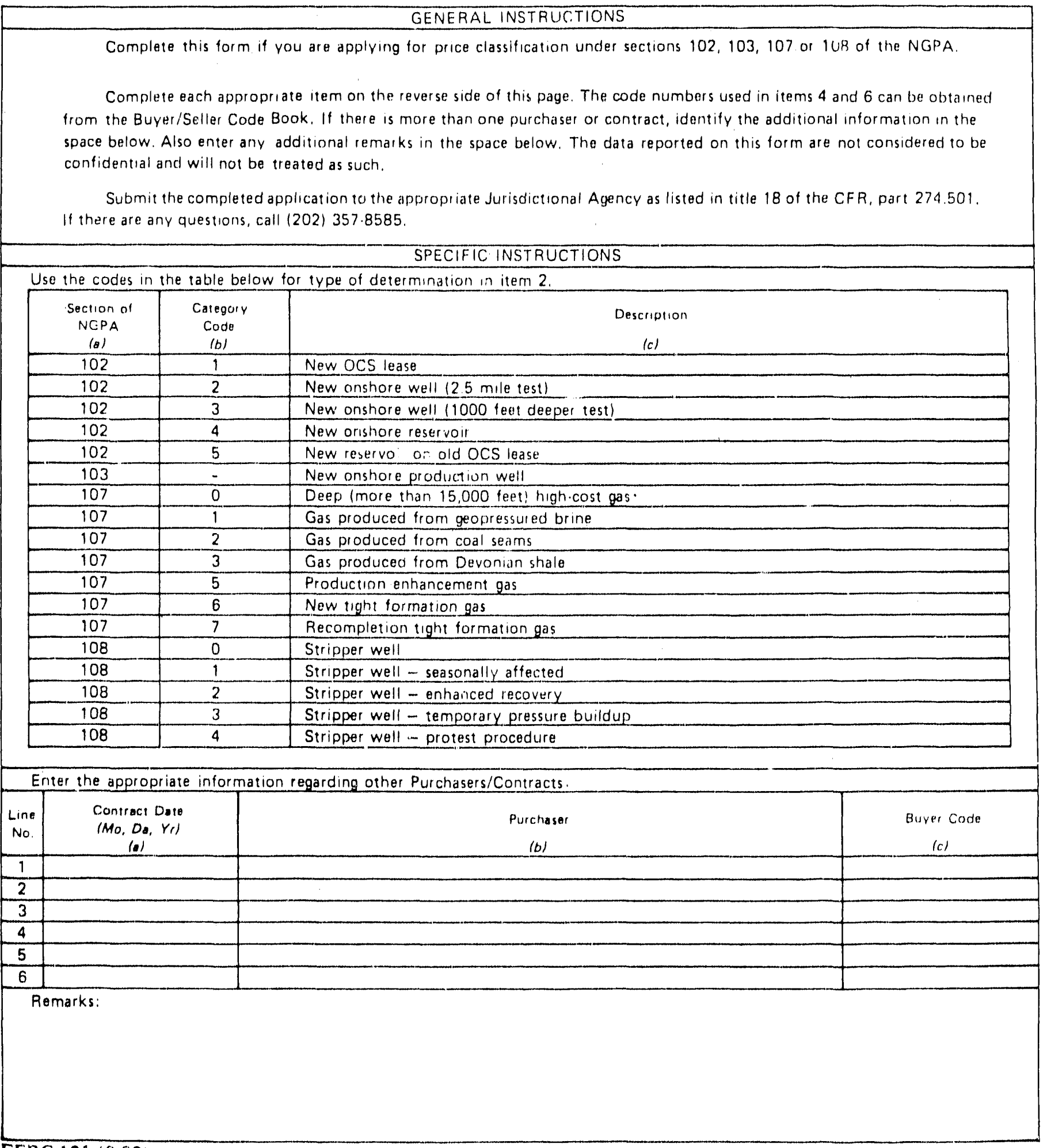


APPLICATION FOR DETERMINATION OF THE MAXINIUM LAWFUL PRICE UNDER THE NATURAL GAS POLICY ACT (NGPA)

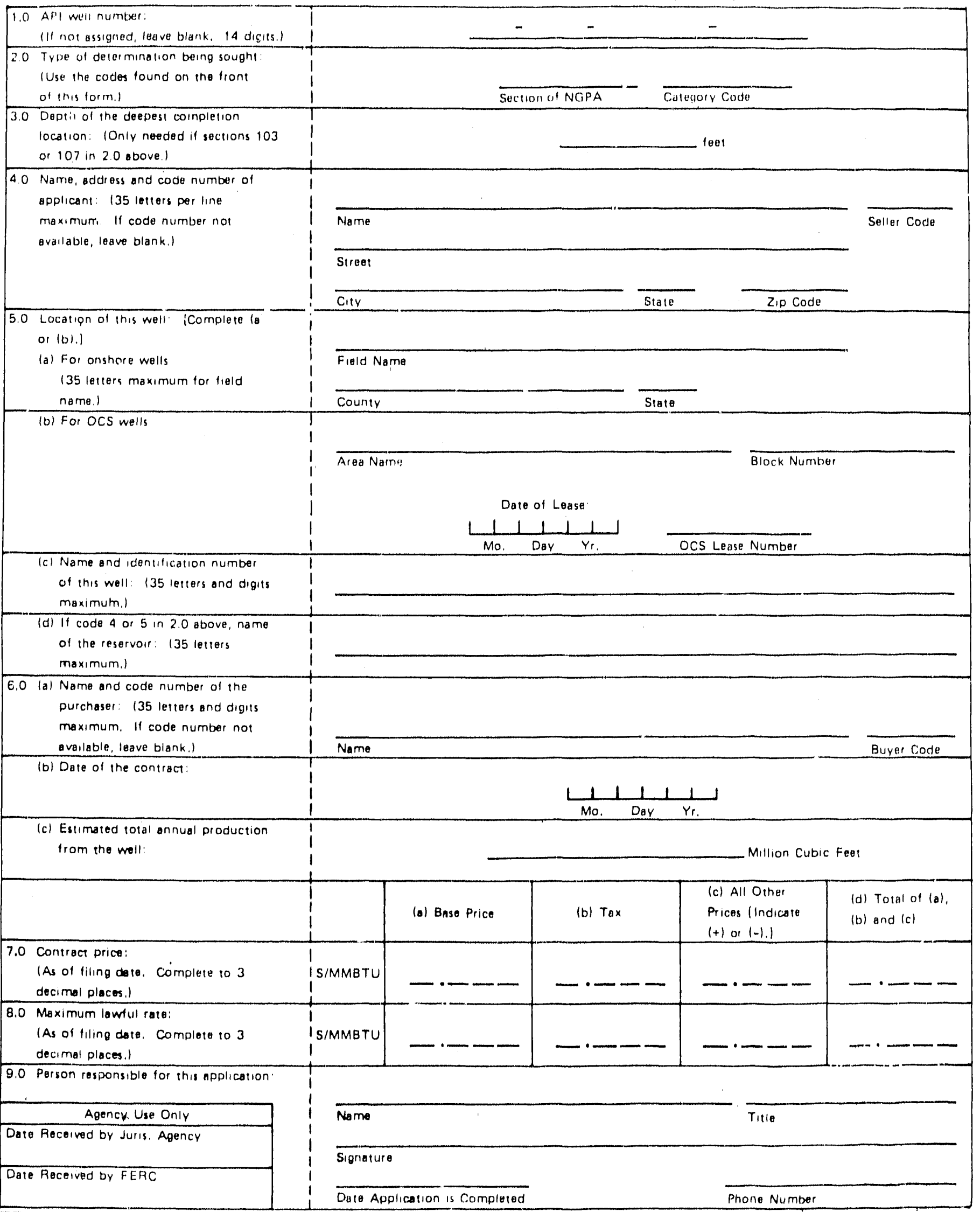

FERC.121(8.82) 


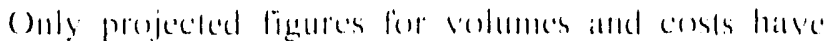

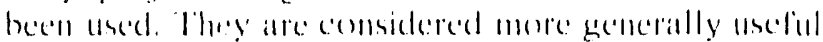

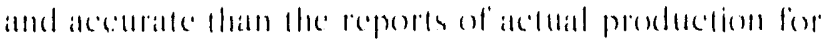
the foliowing reatsoms:

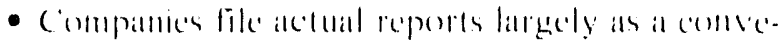

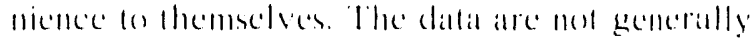
subjected to the same seruting as projections. Moresere pipeline compranies have comsiderable lattatede as fo which time they use in reporting aclatil figures.

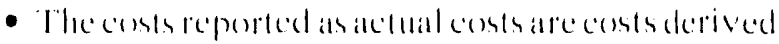
firem previous projections.

- Noll all compranies filled actual fotals.

- As a general rule, meither profeceded ner acenal velumes atre aludited. In the absernce of such an atudit, it is thomght that actual velumes maty be ats subjece lo error as the profecelions.

It appears that Pe is calta have hecome incereasingly accurale and complete with each submission. The mum. bere of carces requiring impulation for missing datal (for

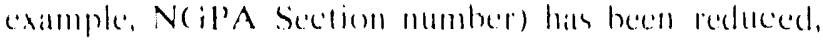

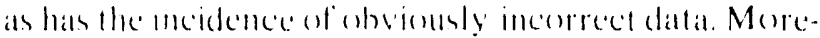
over, complany submissions bate hecome incereasingly comsistent willo catch onther.

Purchased volumes and prices were checked agatost combrol cotals laken from the hard copy of the submission an lite with the I:T:RC: The data comprising incombistent lotale were compared direcely with the hil.

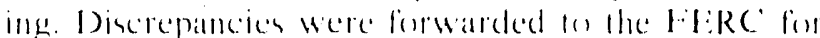

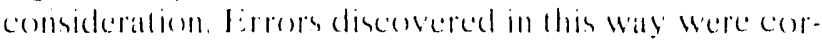
rected, and the prexess was repeated matil agrecement wath reached.

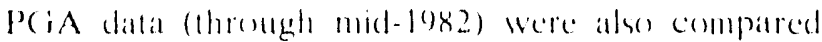

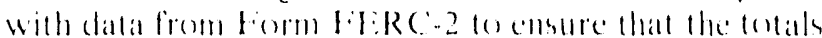
derived from the lwe souteces wete compalible, The awo filings cammon be compared direcelly becalluse the PCis is mol mollatly tiled for callendat years.

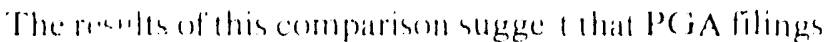

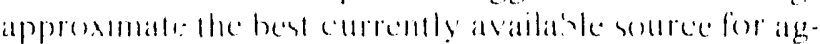
gregale informaltom, bul do mol malch a very chesely.

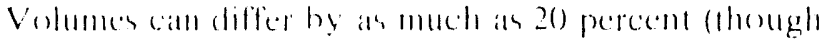

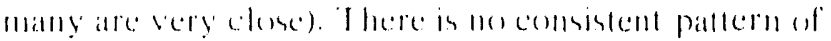
volume differemeses In the case of prices there appeass

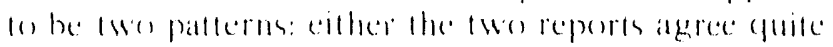

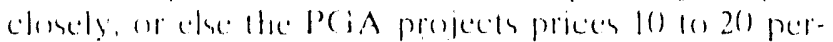

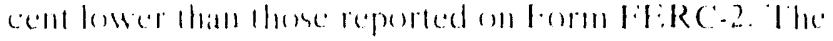

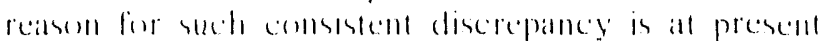

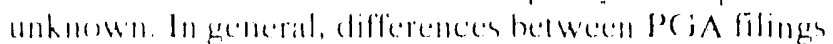
and form 2 information maty be due to a vallety of c:allses, including:

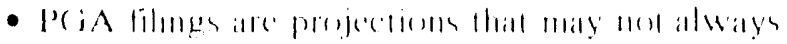

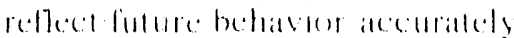

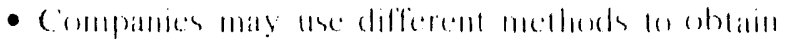
pris propectirims

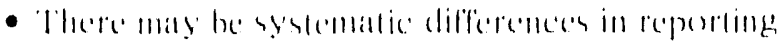

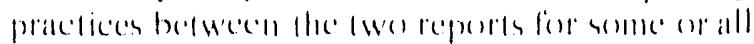
(c)illpantiles.

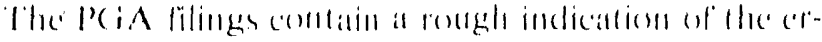

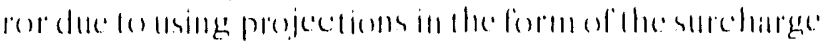

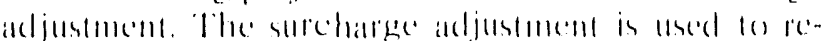

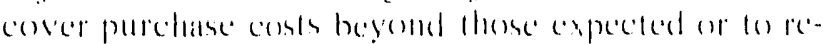

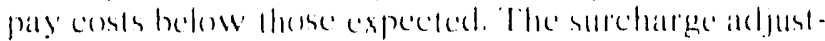

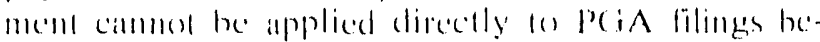
callse:

- Il combanos only a summary ligure for all differences from projected levels. This includes cousts of pipeline purchares and imports.

- Nor volumes are direcelly reporeded 1 cons per llousand cubice feed figure is reported but is based ou projected future purchases anly.

- Ir covers time periends different firem previous projections. Nemally, the surcharge adjustment

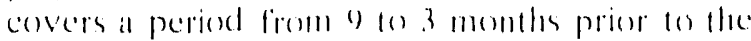
beginning of a new projection periend.

The Pe is filings remano the only source of information for antalyzing gat callegertices, heswever, and the dala problems do nost appear lo be crippling for purposes of drawing genceral conclusions.

No further validation of the P' is datla hats heen performed because of the difficulties involved in such at precess. There are some precedural issues that may alfect the aceuracy of filings, however. Pris filings are not intended lo serve ats datal collection instruments per se but as a regulatory syestem for matural gas. (Daces. lioms concerming the dalla are lostually atred cluring Fl:RC ralle hearings, where the primary concern is reselution of legal issteses. No allempl is made lo revise

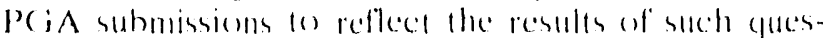
lioning. In addition, most Pe is filings are profested and the resulting tariffis are suspended for a time. In

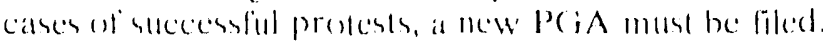
The precise status of data on a given courent submission becomes difficult on judge.

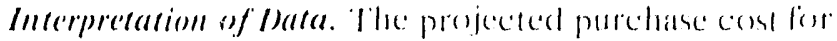
each contract volume represents the pipeline compes

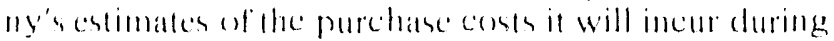
the conerage periond of the filing. These costs include:

- The average price en be paid a the predecer

- Cialhering! charger

- Tramsportation chatgen

- Taxes

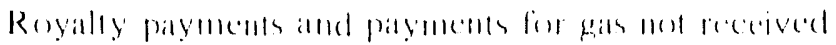

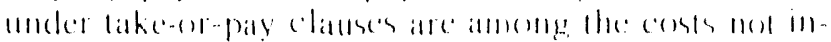
elender

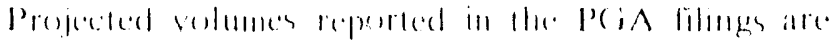

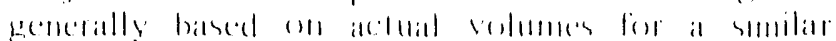


preceding period (though they are typically adjusted (o) lit collrent mattiol conditions). A number of projected gas purethases reported in the P'a filings have not been comsidered hecaluse: they would haver resulted in signilicant druble connting. They are:

- Purchases from other pipelines

- 1 miporis

- Iixchange gats.

In addition, cost-of'-scevice gats (a company's own production from specific leascholds of pre-NGl'A gas) is not comsidered because it is not covered by the NGPA and hecallse most companies do mot include if in theil ' is lilings.

The lable included in this publication is therefore designed lo cover a specilic part of the overall national gas mattice: wellhead purchases that are both subject (1) the Neil'A and dedicated to the interstate mated.

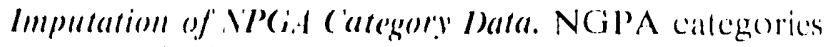
were not included in part or all of some early P(iA filings. To make filings comparable, each contract thal did not have a reported NoipA section was assigned one based on cost. The same procedure was used for reported Neil'A sections when the prices were obviously inappropriate less than lopereent of the volume was subjected to this procedure in any given month. Since the inception of the Form 542-PGA, NGPA callegeries are included in all PCo lilings.

Miscellancens ddjustments. Slight reporting discrepancies among the companies reguired further minot adjustments to the dala base. The adjustments are as follows:

- All volumeric projections have seen converted to monthly ralles.

- In their Peis liling, many companies, in whole or ir art, reported heir gas volumes at a pressure base other than 14.73 psia. Volumes provided at the other pressure hases (14.65 and 15.(2)5) were adjusted to the 14.73 psia level. The factors used in lle adjustment were:

- Volume (all 14.6.5) x(0.9946. Volume (al 14.73)

- Volume (a1 15.025) x 1.02: Volume (al 14.73).

- Velumetric dala are prescrnted in cubic feed, although several companices reported in therms for their hardeopy filings. Volumes in cubic feed alle available on lapec.

- All prices are reported in cents (or dollars and cents) per thomsand cubic foet.

In filings rececived since July 1,1984, all companies are reporting volumes in mi! ion Btu making pressure base acljustments lo volumes moncecessaly

\section{Form FPC-14, "Annual Report for Importers and Exporters of Natural Gas"}

\section{Survey Design}

The collection of data covering matural gas imports and exports was begun in 197.3 by the Federal Power

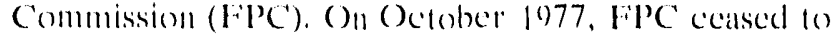
exist and its data collection functions were transferted to the Fecleral Energy Regulatory Commission (FERC) within the Department of Encrgy (DOE), Since 1979, the Energy Information Administration (EIA) has had the responsibility for collecting Form Fil' -14

\section{Survey Universe and Response Statistics}

The Form lape-14 is filed annually by each organization or individual having anthorization $10 \mathrm{impost}$ and export natural gas regardless of whether any imports or exports fook place duing the reporting year. The aulhorization fo import and export was originally granted by the file. In 1977, it was lanferred to the Economic Regulatory Commission (ERA) and it now: resides with the office of Finels leregrams in the Office of Fossil Energy. In 1989, 205 companies met the reporting criteria, only 76 reported import or export of nallural gas.

The respondent list for ihe Form FPC. 14 is updated at the beginning of each year. All new respondents with atuthorization to import or export natural gas are added to the list and respondents whose licenses have expired are deleded. Five copies of Form Fl' -14 are mailed in lebruary lo all companies authorized fo import or export natural gats. The eompleted original and three copies are to be filed with the EIA on or before March 31 of each year, for the preceding calcendar year. Companies that have not filed by March 31 are confacted.

\section{Routine Form FPC-14 Edit Checks}

Respondents are required lo erertify the aceuracy of all clata reported. The survey forms are checked at the LEA for responableness and accuracy. If errots are found the companies are repurired fo file eorrected cata. The dala are proesesed at the EIA and published ats reported. All matural gas volumes in this report are expressed at a pressure base of 14.7 .3 pounde per syuare inch abselule and lemperature of of (legrees Fahrenlecit, except as nofed. All prieces are in U.s. dollars and importexport preces are these paid at the U.S. herder (except experts of I Ne are these prices paid at the point of sale and delivery in Yokohama, Japand). 


\section{"Quarterly Natural Gas Import and Export Sales and Price Report"}

This report is prepared quarterly by the office of Fuels Programs in the Office of Fossil Energy based on information submitted by all firms having authorization to import or export natural gas. All data on this report is considered preliminary until the annual data on the Form FPC-14 is final, usually in seprember of the following year.

\section{Form EIA-857, "Monthly Report of Natural Gas Purchases and Deliveries to Consumers"}

\section{Survey Design}

The original Form ElA-857 was approved for use in December 1984. Response 10 the Form EIA-857 is mandatory on a monthly hasis. Data collected on ihe Form EIA-857 cover the 50 States and the District of Columbia and include both price and volume data.

\section{Survey Universe and Response Statistics}

A sample of 408 satural gas companies including interstate pipelines, intrastate pinelines, and local distribution companies report to the survey. The sample was selected independentiy for each of the 50 States and the District of Columbia. Each selected company is required to complete and file the Form ElA-857 on a monthly basis. Initial response statistics on a monthly basis are as follows: responses received by due date, approximately 9() percent, and responses received after follow-up, 100 percent.

The Form ElA-857 is a monthly sample survey of natural gas marketers, including interstate pipelines, int rastate pipelines, and local distribution companies. It provides data that are used 10 estimate monthly sales of natural gas (volume and price) by State and monthly deliveries of natural gas on behalf of others (volume) by State to three consumer sectors - residential, commercial, and industria!. (Monthly deliveries and prices of natural gas to electric utilities are reported on the Forr: FERC-423, "Monthly Report of Cost and Quality of Fuels for Electric Plants," and the Form ElA-759, "Monthly Power Plant Report.")

See A.ppendix (' for a discussion of the sample design and estimation procedures.

\section{Summary of Form ElA-857 Data Reporting Requirements}

Data collected monthly on the Form EIA-857 on a State level include the volume and cost of purchased gas, the volume and cost of natural gas consumed by sector (residential, commercial, and industrial), and the average heat content of all gas consumed. Respondents file completed forms with EIA in Washingten, DC on or before the 30 th day after the end of the report month.

All natural gas volumes are reported in thousand cubic feet at 14.73 psia at $t()$ degrees Fahren.ir it and dollar values are reported to the nearest whole dollar.

\section{Routine Form EIA-857 Fdit Checks}

A series of manual and computerized edit checks are used to screen the Form EIA-857. The edits performed include validity and analytical checks 


\section{MONTHLY REPORT OF NATURAL GAS PURCHASES AND DELIVERIES TO CONSUMERS}

This report is mandatory linder the Federal Energy Administration Act of 1974 (Public Law 93-275). Fallure fo report may result in criminal fines, civil penalties, and other sanctions as provided by law. See section VI of the instructions for the confidentiality statement. Public reporting burden for this sollection of information is estimated to average 4 hours per response, including time of reviewing instructions, searching existing data sources, gathering and maintaining the data needed, and completing and jeviewing the collection of information. Send comments regarding this burden or any cther aspect of this collection of information, inc'uding suggestions for reducing this burden, to the Energy lnformation Administration, Office of Statistical Standards EI-73, Mall Station: 1 H.023 Forrestal, 1000 Independence Ave. SW, W'ashington, DC 20585 ; and to the Office of Information and Regulatory Affairs, Office of Management and Budget, Washington, DC 20503.

Affix Indentification Label or Enter:

01 Control No.

02 Company Name:

State of Operation:

SECTION I - Natural Gas Purchases and Deliveries to Consumers

Purchased Gas Received in Distribution Service Area

Delivered to Residential Consumers (Onsystem Sales)

Delivered to Commercial Consumers (Onsystem Sales)

Delivered to Industrial Consu wers (Onsystem Sales)

Transported to Commercial Consumers

Transported to Industrial Consumers

A verage Btu Content of Gas Delivered to Consumers

SECTION 【 - FOOTNOTES
Return completed forms to:

Energy Ittormation Administration

Mail Station: BG-094

U.S. Department of Energ:

Washington, D.C. 20585

Attn: Form ELA-857

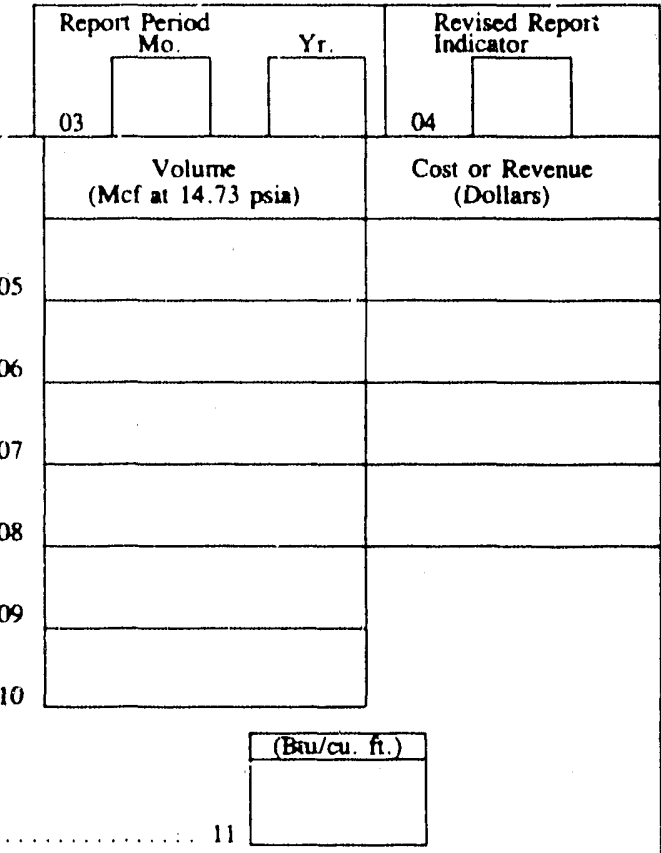

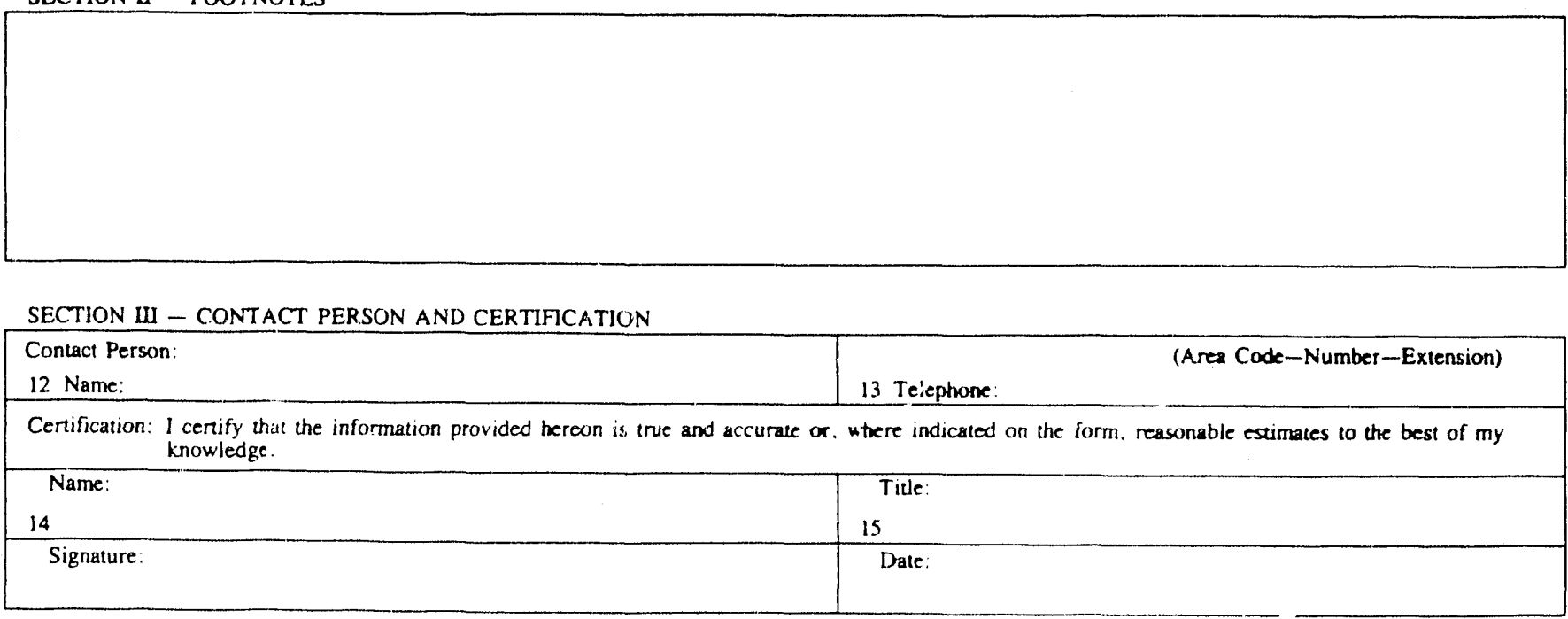


FEDERAL ENERGY REGULATORY COMMISSION Washington, D.C. 20426

\section{ANNUAL REPORT FOR

1. The completed original and 3 conformed copies of this report shall be filed with the Foderal Energy Regulatory Commission, Washington, D.C. 20426 on or betore March 31, of mach vear, for the preceding calendar vear.

2. The report will be filed by each person having authorization to import or export ratural gas.

3. Use separate schedule for each authorization. If one authorization involves more than one import or export point, a separate schedule must be filed for each point.

4. All volumes reported in Met will be at 14.73 Psia and $60^{\circ} \mathrm{F}$.

5. Where transactions are based primarily on volumetric measurement, weighted average Btu data should be used to estimate total morithly Biu's.

6. Amounts paid of received and cost or receipts shall be reported in U.S. Dollars.

7. DEFINITIONS: - Transporter - the party or parties, other than buyer or seller, owning the facilities by which gas or LNG is physically transferred between buyer and seller; Costs-all expenses incurred by importer up to the U.S. point of delivery for the reported quantity imported; Receipts-all revenues received by exporter for the reported quantity exported.

\begin{tabular}{|c|c|c|c|c|c|}
\hline \multicolumn{3}{|c|}{ Name of Respondent } & \multicolumn{2}{|c|}{ Address of Respondent } & $\begin{array}{l}\text { Year Ending } \\
\text { December } 31 .\end{array}$ \\
\hline \multicolumn{6}{|c|}{ SCHEDULE I - GASEOUS PHASE NATURAL GAS } \\
\hline \multicolumn{6}{|c|}{$\begin{array}{l}\text { NOTE: If autharization granted is increased, decreased, or extended under a succession of docket numbers and all } \\
\text { related gas moved through the came border facilities and authorized expansions thereof, moved to or from the same } \\
\text { purchaser or supplier, and was subject to the same rate, such transaction shall be combined for reporting purposes and } \\
\text { appropriate Docket Numbers shown. }\end{array}$} \\
\hline \multicolumn{2}{|c|}{$\begin{array}{l}\text { Report for (Check one) } \\
\square \text { Import } \square \text { Export }\end{array}$} & \multicolumn{4}{|c|}{ Foreign Seller or Foreign Buyer } \\
\hline \multicolumn{2}{|c|}{ U.S. Entry or Exit Point } & & Transporter & & Docket Number(s) \\
\hline \multirow{3}{*}{$\begin{array}{l}\text { Line } \\
\text { No. }\end{array}$} & \multirow{3}{*}{$\begin{array}{l}\text { Month } \\
\text { (a) }\end{array}$} & \multicolumn{3}{|c|}{ QUANTITY RECEIVED OR SHIPPED AT THE ENTAY OR EXIT POINT } & \multirow{3}{*}{$\begin{array}{l}\text { Amount Pard or } \\
\text { Received ot Entry } \\
\text { or Exit Point } \\
\text { (fi) }\end{array}$} \\
\hline & & \multicolumn{2}{|c|}{ Paso Dor } & \multirow[b]{2}{*}{$\begin{array}{l}\text { Mct } \\
\text { (d) }\end{array}$} & \\
\hline & & $\begin{array}{l}\text { Dare } \\
\text { (b) }\end{array}$ & $\begin{array}{l}\text { Met } \\
\text { (c) }\end{array}$ & & \\
\hline 1 & January & & & & \\
\hline 2 & February & & & & \\
\hline 3 & March & & & & \\
\hline 4 & April & & & & \\
\hline 5 & Mar & & & & \\
\hline 6 & June & & & & \\
\hline 7 & July & & & & \\
\hline 8 & August & & & & \\
\hline 9 & September & & & & \\
\hline 10 & October & & & & \\
\hline 11 & November & & & & \\
\hline 12 & December & & & & \\
\hline 13 & & & TOTAL & & \\
\hline \multicolumn{4}{|c|}{$\begin{array}{l}\text { ANNUAL WEIGHTED AVERAGE IN BIU's PER CUBIC FOOT } \\
(\text { Line } 13 \text { : (Cotumn }(0) \div(\text { Column }(d) \times(000)))\end{array}$} & \multicolumn{2}{|c|}{$\begin{array}{l}\text { ANNUAL WEIGHTED AVERAGE PRICE IN CENTS PER MMBiU } \\
(\text { Line } 13 ; \text { Column }(f) \div \text { Column }(e) \times(00)\end{array}$} \\
\hline
\end{tabular}

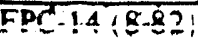


Appendix C

Statistical

Considerations 


\section{Appendix C}

\section{Statistical Considerations}

The monthly sales (volume and price) and monthly deliveries (volume) of natural gas by State presented in this report are estimated from data reported on the Form EIA-857, "Monthly Report of Natural Gas Purchases and Deliveries to Consumers." (See Appendix $B$ for a description of this Form.) These estimations must be made from the reported data since the Form EIA-857 is a sample survey. A description of the sample design and the estimation procedures is given below.

\section{Sample Design}

The Form EIA-857 is a monthly sample survey of natural gas marketers including interstate pipeline companies, int rastate pipeline companies, producers, and local distribution companies. It provides data that are used to estimate monthly onsystem sales of natural gas (volume and price) by State and monthly deliveries of natural gas (volume) by state to three consumer sectors--residential, commercial, and industrial. Monthly deliveries and prices of natural gas to electric utilities are reported on the Form EIA-759, "Monthly" Power Plant Report," and the Form FERC-423, "Monthly Report of Costs and Quality of Fuels for Electric Plants."

Sample Universe. The sample currently in use was selected from a universe of 1,746 companies. These companies were respondents to the Form EIA-176, "Allnual Report of Natural and Supplemental Gas Supply" and Disposition." for reporting year 1988. (See Appendix $B$ for a description of the Form EIA-176.)

Sampling Plan. The goal was a sample that would provide estimates of monthly natural gas consumption by the three consuming sectors within each State and the District of Columbia. A stratified sample using a single stage and sysiematic selection with probathility proportional to size was designed. The measure of size was the volume of gas ates and deliveries by the company during 1988. There were $\left.1 w^{\prime}\right)$ strata-companies selected whthertanty and empondes selected ander the systematic probability proportional to size design.
Initial calculations showed that a 25 pereent simple of companies would yield reasonably accurate estimates. The sample was selected independently in each State. resulting in a national total of 408 companies.

Certainty Stratum. Since estinates were needed for each of the 50) States and the District of Columbia, the strata were established independently within each State. In 16 States and the District of Columbia where sampling was not feasible due to small numbers of companies and/or small volumes of gas sales, all companies were selected. The 16 States were: Alaska, Connecticut, Delaware, Hawaii, Idaho, Maine, North Datkota, New Hampshire, New Jersey, Nevada, Oregon, Rhode Island, South Dakota, Utah, Vermont, and Washington.

For each of the remaining States, the total volumes of industrial sales and deliveries and of the combined residential/commercial sales were determined. Companies with gas sales/deliveries to the industrial sector or to the combined residential/commercial sector above a certain level were selected with certainty. This procedure ensured that large companies would be included since a few large companies often account for most of the volume of gas sales within a State. The formula for determining certainty was applied independently in the two consumer sectors-the industrial and the combined residential/commercial. These selected companies, together with the companies in the jurisdictions discussed where sampling was not feasible, formed the certainty stratum.

All companics with gas salce/deliveries in sector $j$ greater than the cut-off value $(C, j)$ were included in the ecrtainty stratum. The formula for $C$, was:

$$
\text { (.) } \frac{X_{j}}{2 n}
$$

where:

(i) culoff value for comsumet sector is

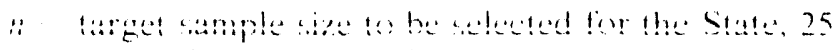
percent of the companices in the Statce. 


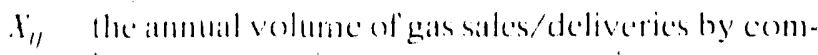
pany i fo customers in comsumer secotor j.

I. He ston within Statce of anmual gats volumes for comprally i.

d, the stmm within State of anmual gats volumes in comsumet ses:l(n) j.

$l^{\prime}$ Hhe stum within Stalle of anmual gas velumes in all comsumer sectors

Noncertainty Stratum. All onher companies formed the moncertainty stratum. They were systematically sampled with probability proportional fosize. The measure of size for cach company was the total volume of gas sales to all comsumer secelors $\left(x_{1}\right)$. The number of compantes to be selected from the: noncertanty stratum was calculated for cach State, with a minimum of 2

The formula for selecting the number of noncertainty stratum compranices was:

$$
m \quad 11 \frac{12}{1}
$$

where:

$m$. the sample size for the noncertainty stratum within a Stale.

$X^{2}$... the sum within State of the $X$, for all companies in the noncertainly stratum.

Companies were listed in ascending order according to their measure of size and then a cumulative measure of size in the stratum was calculated for each company. The cumulative measure of size was the sum of the measures of size for that company and all preceding companies on the list. All interval (I) for selecting the companices systematically was calculated $\left(I \ldots \frac{X 2}{m}\right)$. A uniform random number $\mathrm{R}$ was selected between zero and $I$. The first sampled company was the first company on the list to have a cumulative measure of size greater than $\mathrm{R}$. The second company selected was the first company on the list to have a cumulative measure of size greater than $\mathrm{R}+\mathrm{I}$ R + I was increased again by I to determine the third company to be selected. This procedure was repeated until the entire sample was drawn

Subgroups. In 12 Stalles, the noncertainty stratum was divided inte subgroups to ensure that gas in each (ont)sumer secter could be estimated. The systematic sample with probahility proportional fo size design described aboue was applied independently in each subgroup. The methesh for determining the subgroup sample size and calculating the subgroup interval for samnle selection were the same as the methods described abere for the moncertanty stratum. except that X2 was the sum within State of the $x_{i}$ lor only those compantes in lle subgr(oup).

These subgremps mere defined only for the purpose of sample sefection. 'They are:

West Virginia: companics handling only residential/commercial gas and all olhere compalnics.

California, 1 ousisiana, and Mississippi: compantes handling (nly industrial gas and all other companics.

Alabama, Illinosis, Kentucky, and Ohios compa nic's handling any industrial gas and all ofher companics.

Texas: companics handling only residential/ commercial gas, companies handling only industrial gas, and all other companies (three subgroups).

Arkansas: (ompantes handling (0.5 million cubic feet or more of gas and companics handling less than ().5 million cubic feet of gas.

Colorado: companics handling 1 million cubic fece or more of gas and companies handling less that 1 million cubic feet of gas.

New York: companies handling 8 million cubic fect or more of gatas and compantes handling less than 8 million cubic fece of gas.

\section{Estimation Procedures}

Estimates of Volumes. A ratio estimator is applied o the volumes reported in each stale by the sampled companies to estimate the total gas sales and deliveries for the State. Ratio estimators are calculated for cach consumer sector-eresidential, commercial, and industrial--in each State where companics are sampled. The following annual data are laken from the most recent 1989 submissions of Form EIA-176:

The formula for calculating the ratio estimator $\left(E_{v j}\right)$ for the velume of gats in consumer secelor j is:

$$
E_{y}-\frac{y_{j}}{Y_{j}^{\prime}}
$$

where:

$Y_{. j}$ the sum within Statc of anmual gats volumes in consumer sector f for all companics,

$y^{\prime \prime}$. The sum within Slate of anmual gats velumes in consumer serefor for flose companies in the sample. 
The ratio estimator is applied as follows:

$$
V_{j}=y_{1} \times E_{y j}
$$

where:

$V_{j}=$ the State cstimate of monthly gas volumes in consumer sector $\mathrm{j}$,

$y, j=$ the sum within State of reported monthly gas volumes in consumer sector $\mathrm{j}$.

Estimates of Revenues. State reventes are estimaled from monthly data reported by the sample companies in the same way as the volumes are estimated. Ratio estimators are calculated for each consumer sector based on annual sales revenue in each State where companies are sampled. The estimated reventues are subsequently used to calculate average prices.

The formula for calculating the ratio estimator for residential gas sales revenues in consumer sector $j$ is:

$E_{\eta j}=\frac{Z_{. j}}{Z_{. j}^{\prime}}$

where:

$Z_{j}=$ the sum within State of annual gas sales revenues in consumer seclor j.

$Z_{. j}^{1}=$ the sum within State of annual gas sales revenues for those companies in the sample in consumer sector j.

The ratio estimator is applied as follows:

$R_{j}=z_{j}, E_{j}$

where:

$R_{j}=$ the monthly estimated revenue for gas sales in consumer sector $\mathrm{j}$.

$z_{1}$ the sum within State of reported monthly gats satles reventues in consumer sector $\mathrm{j}$.

Computation of Natural Gas Prices. The natural gas volumes that "acluded in the computation of prices represent only those volumes associaled with natural gails sules.

The average price of natural gas is calculated as follows:

$P \quad \frac{R}{r}$ where:

$P_{j}=$ the average price of gals sales within a State in consumer sector $\mathrm{j}$,

$V_{r}=$ the estimate of gas sales within a State in consumer sector j.

All average prices are weighted by their corresponding sales volume estimates when national average prices are computed.

The monthly average prices of natural gats in the commercial and industrial sectors are based on sales data only. Volumes of gas delivered for the account of onhers to these consumer sectors are not included in the State or national average prices. Virtually all natural gas deliveries to the residential sector represent sales volumes only.

Table 3.3 shows the percent of the total State volume that represents volumes from natural gas sales to the commercial and industrial sectors. This table may be helpful in evaluating commercial and industrial price data.

Estimation for Nonrespondents. A volume for each consumer category is imputed for companies that fail to respond. The imputation is based on the previous month's value reported by the non-responding company and the change from the previous month to the current month in volumes reported by other companies in the State. The imputed volumes are included in the State totals. To estimate prices for non-respondents, the unit price (dollars per thousand cubic feet) reported by the company in the previous month is used.

The formula for imputing volumes of gas sales for nonrespondents was:

$$
F_{1}=F_{i}, \frac{y_{j}}{y_{, j} t-1}
$$

where:

If.-. imputed gas volume for current month t,

$F_{i}$, gats volume for the company for the previous month,

b.y gas volume reported by companies in the Statle stratum for report momth 1 .

y.j 1 gas velume in the previous month fore com-

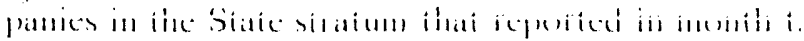




\section{Final Revisions}

Adjusting Monthly Data to Annual Data. After the anmual datat reported on the Ferm EIA-176 have been submitted, edited, and prepared for publication in the

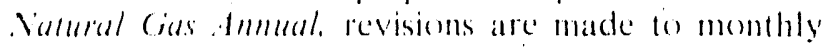
data. The revisions atre made to the volumes and prices of mallual gas delivered fo comstumers that have appeated in the Nantral (ios Momelhly to match them to the annual values appearing in the Nanural cids dmmual. The revised mombly estimates allocate the difference between the sum of momthly estimates and the ammoal reports aceording to the distribution of the cestimated values atcoss the momiths.

Before the final revisions are made, changes or additions fo submitled dala receseded after publication of the mombly estimate and mot sufficiently latge to require a revision an he publisted in the Nalural (jas Momhly are used lo derive an updated estimate of mombly consumption and reventes for each State's residential, commercial, or industrial natural gats comsumption.

For cach State, wo mumbers were revised, the estimaled comsumption and the estimated price per thou. sand cubic feet.

The formula for revising the estimated comsumption is:

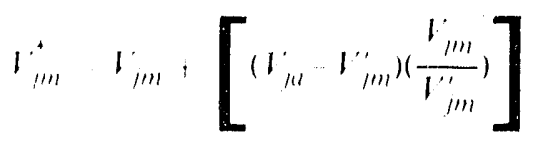

What:

$L_{\text {min }}^{*}$ the final velume estimate for month $m$ in consumer sector i.

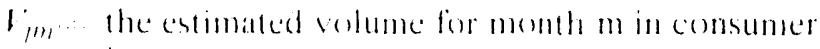
sector is.

$V_{11}$ the volume for the year reported on Form IIIA-176.

$l^{\prime \prime \prime m}$ The ammual sum of estimated momthly volumes.

The price is calculated as described above in the Estimation Precedures section, using the final revised consumption estimate and a revised revente estinate.

Ihe formula for revising the entmated revenue is:

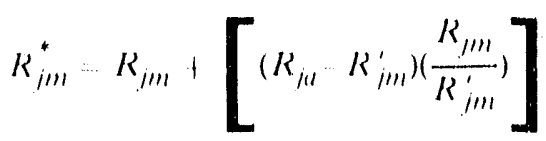

where:

$R_{j m}^{*}$ the final revenue estimate for momth $m$ in eorm. stumer sector j,

$R_{j m}$ the estimated revente fin month min consumet sectori. i.

$R_{j u}$ : the reventic for the yeat reported on form EIA-176.

$R_{j m}^{\prime}$ The anmual sum of estimated monthly revenues.

\section{Reliability of Monthly Data}

The monthly data published in this report are subject to two sources of error - nomsampling error and sampling error. Nonsampling errors occur in the collection and processing of the data. Sec the discussion of the Form ElA-857 in Appendix B for a description of nomsampling corors for monthly data.

Sampling error may be defined as the difference between the results obtained from a sample and the results that a complete enumeration wesuld provide. The standard error statistic is a measurement of sampling error.

\section{Standard Errors}

A standard error of an estimate is a statistical measure that indicates how the estimate from the sample compares 10 the result from a complete enumeration. Standard errors are calculated based on statistical theory that refers to all possible samples of the same size and design.

The standard errors for monthly matural gas volume estimales by State are given in Table (2. Nincty-five percent of the time, the velume that would have been (b) anded from a complete enumeration will lie in the range between the estimated velume minus two standard errors and the estimated velume plus lwo standard errors. 
Table C-1. Standard Error for Natural Gas Deliveries and Price to Consumers by State, August 1990

\begin{tabular}{|c|c|c|c|c|c|c|c|c|}
\hline \multirow{2}{*}{ State } & \multicolumn{4}{|c|}{$\begin{array}{l}\text { Volume } \\
\text { Million Cubic Foed }\end{array}$} & \multicolumn{4}{|c|}{$\begin{array}{c}\text { Prico } \\
\text { Dollars per Thousand cuble: Foul }\end{array}$} \\
\hline & Rosiddential & Commorcial & Industriat & Tolial & Rosidenlial & Commorcaal & Indusilmal & lolal \\
\hline Alabama ............. & 146 & 58 & 450 & 483 & 069 & $02 \%$ & 0.31 & () 44 \\
\hline Alaska & 0 & 0 & 0 & () & 00 & 00 & .00 & .00 \\
\hline Arizona & y & 8 & 0 & 12 & 00 & 01 & 00 & 04 \\
\hline 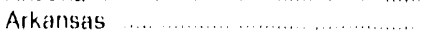 & 25 & 27 & 546 & $5,4 \%$ & 1,3 & 04 & 45 & 54 \\
\hline 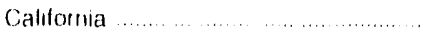 & 344 & 328 & 353 & 593 & 07 & .04 & .01 & .06 \\
\hline Colorado ..... & 15 & $2 ?$ & 790 & 791 & 96 & $0 \%$ & 94 & .83 \\
\hline Connecticul & 0 & 0 & 0 & 0 & 00 & 00 & 00 & 00 \\
\hline Delaware & 0 & 0 & 0 & 0 & .00 & .00 & 0() & .00 \\
\hline District of Columbia ........................... & 0 & 0 & 0 & 0 & 00 & (0) & 0() & .00 \\
\hline 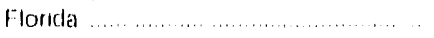 & 29 & 136 & 597 & 613 & 22 & 09 & 46 & in \\
\hline 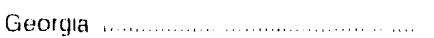 & 128 & 203 & 700 & 741 & $\therefore 0$ & 40 & $3 \%$ & .71 \\
\hline Hawall & 0 & 0 & 0 & () & .00 & .00 & 00 & 00 \\
\hline Jdaho & 0 & 0 & 0 & 0 & .00 & 00 & .00 & 00 \\
\hline Illinoss & 180 & 301 & 61 & 356 & 10 & .24 & $0 ?$ & 13 \\
\hline 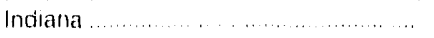 & 28 & 74 & 760 & 764 & .58 & 31 & .16 & 23 \\
\hline 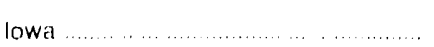 & $: 1$ & 8 & 32 & 33 & $0 ?$ & .01 & $0 ?$ & . ()9 \\
\hline Kansas ............... & 0 & 0 & 0 & 0 & 00 & .00 & 00 & 00 \\
\hline 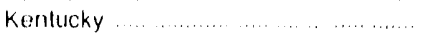 & 33 & 76 & 105 & 134 & .16 & .06 & 66 & 1.14 \\
\hline 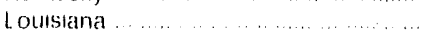 & 19 & 4 & 13,714 & 13.714 & .30 & .01 & 05 & 1.00 \\
\hline 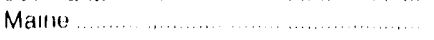 & 0 & 0 & 0 & 0 & .00 & .00 & .00 & .00 \\
\hline 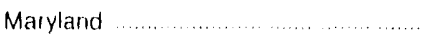 & 9 & 8 & 99 & 100 & 01 & 02 & ני & 28 \\
\hline 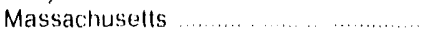 & 13 & 118 & 723 & 732 & 20 & .34 & 04 & 37 \\
\hline 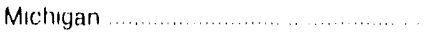 & 339 & 300 & 3,411 & 3,441 & .15 & .08 & 19 & 33 \\
\hline 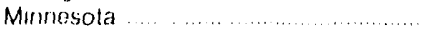 & 155 & 80 & 646 & 669 & .46 & 1', & $1 ?$ & .25 \\
\hline Mississippi . ................................ & 127 & 118 & 842 & 859 & .06 & 15 & 19 & 152 \\
\hline 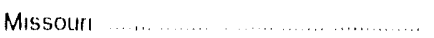 & 121 & 188 & 87 & 240 & .03 & 13 & .08 & 25 \\
\hline Montana & 2 & $?$ & 0 & 2 & .01 & 01 & .00 & .02 \\
\hline 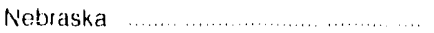 & 20 & 1913 & 85 & 216 & 08 & .01 & .06 & .23 \\
\hline 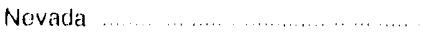 & 0 & 0 & 0 & 0 & .00 & .00 & .00 & .00 \\
\hline 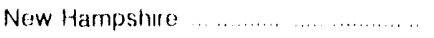 & 0 & 0 & 0 & 0 & .00 & .00 & .00 & .00 \\
\hline 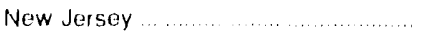 & 0 & 0 & 0 & 0 & .00 & .00 & .00 & .00 \\
\hline 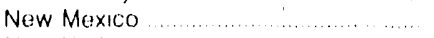 & 52 & 262 & 0 & 267 & .25 & 101 & .00 & 1.22 \\
\hline 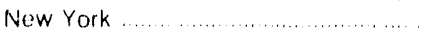 & 390 & 1.250 & 1,526 & 2,011 & .32 & .04 & .32 & .58 \\
\hline 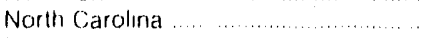 & 9 & 10 & 307 & 308 & 11 & 02 & 15 & 39 \\
\hline 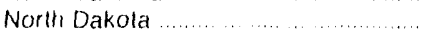 & 0 & 0 & 0 & 0 & .00 & .00 & 00 & Do \\
\hline 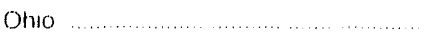 & 242 & 124 & 8,306 & 8,310 & .05 & $.0 \%$ & 11 & 17 \\
\hline Oklahoma & 152 & $9 ?$ & 23 & 179 & 99 & .09 & 02 & 14 \\
\hline 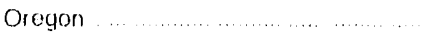 & 0 & 0 & 0 & 0 & 00 & .00 & 00 & .00 \\
\hline Penns) Ivania & 27 & 123 & 776 & 787 & .04 & .13 & 92 & .83 \\
\hline 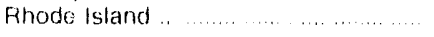 & 0 & 0 & 0 & 0 & .00 & .00 & 00 & 00 \\
\hline South Carolına & 23 & 94 & y24 & 929 & 52 & .03 & 22 & 36 \\
\hline 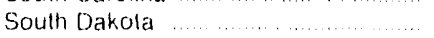 & 0 & 0 & 0 & 0 & 00 & .00 & 00 & .00 \\
\hline Tennessee & 54 & 70 & 793 & 798 & 10 & 09 & 13 & .55 \\
\hline Texas & 60 & 14.7983 & $10,7,7$ & 18,307 & 01 & 2.20 & $1 ?$ & .90 \\
\hline 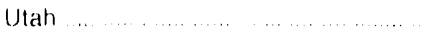 & 0 & 0 & 0 & 0 & 00 & 00 & .00 & .00 \\
\hline Vermont & 0 & 0 & 0 & 0 & .00 & .00 & .00 & 00 \\
\hline Virgirua $\ldots \ldots \ldots \ldots$ & 52 & 33 & 665 & 672 & 10 & .17 & .55 & 95 \\
\hline 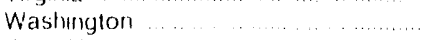 & 0 & 0 & 0 & 0 & .00 & .00 & 00 & .00 \\
\hline West Virginia $\ldots \ldots \ldots$ & 19 & 46 & ? & 49 & 55 & 16 & 00 & 28 \\
\hline Wiscorisin & 43 & $10 ?$ & 564 & 574 & 33 & (1) 5 & 12 & 16 \\
\hline 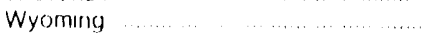 & 11 & 69 & 71 & 100 & .37 & 836 & 19 & 86 \\
\hline U.S Tolals & $783 ;$ & 14,871 & 19,862 & 24,825 & 06 & 32 & 17 & 16 \\
\hline
\end{tabular}


The standard errof of the nalleral gas volume estimate is the sequate root of the variance of the estimate. The formula for calculating the vartance of the volume ess. timate is:

$$
\text { (i) } \sum_{n=1}\left[N_{i}^{2} \frac{\left(1-\frac{n_{1}}{N_{1 /}}\right)}{n_{1 /}\left(n_{h}-1\right)}\left(\sum_{1}\left(v_{i}-T x_{i}\right)^{2}\right)\right]
$$

where:
$N_{h}$ the total number of companies in stratum h

$11 / \cdots$ he sample size in strutum h

$v_{i}$. the reported monthly volume for compuny i

$x_{1}, \ldots$ the reperted anmual volume for company i

$T$... the ratio of the sum of the reported monthly volumes for sample companies to the stom of the reported annual volumes for the sample companies. 
Appendix D

Natural Gas

Reports and

Feature Articles 


\section{Appendix D}

\section{Natural Gas Reports and Feature Articles}

\section{Reports Dealing Principally with Natural Gas and/or Natural Gas Liquids}

- Natural Gias Ammual 1989. DOEE/EIA-(01.31(89). Seprember 190().

- Stanistics of Imterstate Natural Gias Pipelime Companies 1988. DOE:-EIA-(0)45(88), November 1989. Published annually.

- Gass Suppliess of Interstate Natural Gas Pipalime Companies 1988, DOE:L1A-0167(88), Decemher 1989. Published annually.

\section{Other Reports Covering Natural Gas, Natural Gas Liquids, and Other Energy Sources}

- U.S. Crude Oil, Nanural Gas, and Natural cias Liquids Rescerves - 1989 Allmual Repurt. DOE:EIA-(0216(89), September 199),

- Momhly Energy Review, DOE/EIA-()035. Published monthly. Provides national aggregate dala for matural gats, natural gats licquids, and other encergy sources.

- Ammual Roporr a Congress 1989. DOEEa EIA-(0)7.3(89), March 199(). Published annually.

- Ammual Eincrgy Oullook l990, DOE/ EIA-().383(9)()), January 196)(). Published annually.

- Anmual linergy Review 1989, DOE: EIA-0.384(89), May 1990). Published annually.

- Short-Term Energy Oullook, DOE/EIA-()2(1)2. Published quarterly. Provides forceasts for next six quarters for natural gas and other encrgy sources.

- Anmual Oullook for Oil and (ias: logo DOEL IiA-()517()(0), May 19(0).

- International Oil and Cias Exploration and Development Activities, DODE/EIA-(0523. Published (Quarterly. Provides a compilation of reported oil and gas reserve additions in forceign countries.

\section{Selected One-Time Naturail Gas and Related Reports}

- Drilling and Production Under Title I of the Natural Gias Policy de' 1978-1986, DOE/EIA-()448(86), January 1989.

- An Examination of Domestic Natural Gas Resource Estimates, SR/RN(GD/89-()), Fubruary 1989.

- Girowth in Unbundled Natural Gas Tramsportation Services: 1982-1987. DOE:LIA-0525, May 1989.

- Assessmont of Pipeline C'apacity to Transport Domestic Natural Gias Supplies: to the Northe'ast. SR/RNGD/89-()2, August 1989.

- Natural Cias Production Responsess os a Changing Marker Einvironment. 1978-1988. DOE/ LIA-(15.32, June 199).

- Costs and Indices for Domestic Oil and Gas Fïeld Eequipment and Production Operations - 1987 through 1989, DOE/EIA-(0185(89), July 199().

- U.S. Oil and Gas Reserves by Year of Fïcld Discovery. DOE:LEIA-()5.34, August 1990).

\section{Selected and Recurring Natural Gas and Related Data Reference Reports}

- Directary of Eincrgy Data Collection Liorms, DOE/ EIA-(249)(89), January 1990).

- Oil and cias fiold code Master List, 1989. EIA-(0.37()(89), January 199)( 
NGM Feature Articles

\section{March 1987}

Estimated Distribution of Proved Natural Gas Reserves Among the Geologic Provinces of the Lower 48 States

(Presents an overview of the methodology and procedures employed in the formulation of eertain positions of EJA's anmual reserves study.)

\section{April 1987}

Contract Provisions Covering Production of New Gas

(Discusses various types of contract provisions included in recent nutural gas contracts.)

\section{May 1987}

\section{U.S. Imports and Exports of Natural Gas 1986}

(Provides final 1986 data on all U.S. imports and exports of natural gas.)

\section{July 1987}

\section{Underground Storage of Natural Gas}

(Presents an analysis of the natural gas storage facilities and statistic's.)

Domestic Natural Gas Reserves and Production Dedicated to Interstate Pipeline Companies, 1986

(Provides preliminary data on natural gas reserves and production dedicated to the interstate market.)

\section{October 1987}

Main Line Natural Gas Sales to Industrial Users, 1986

(Describes and analyzes direet sales to end users by interstale pipeline companies and SIC codes.)
Decembrar 987

Overview of Wellhead Purchases by Interstate Natural Gas Pipeline Compan'es Since the NGPA

(Presents an overview of gas purchinsing trends and practices since 1979.)

January 1988

Gas Deliverability in Louistana

(Provides information on the levels of matural gas defiverability in the State of Lomisiana.)

\section{April 1988}

Overview of Pipeline Take-or-Pay lexposure: $198{ }^{x}-1987$

(Presents information on the recent history of the lakeor-pay issue.)

\section{June 1988}

Domestic Natural Gas Reserves and Production

Dedicated to Interstate Pipeline Companies, 1987

(Provides preliminary data on natural gas reserves and production dedicated to the interstate market.)

\section{July 1988}

\section{U.S. Imports and Exports of Natural Gas - 1987}

(Contains final 1987 data on all U.S. imports and exports of natural gas.)

\section{September 1988}

Status of Coalbed Methane Recovery in the United Stutes

(Presents an overview of enalbed methane gas in the U.S.) 
Main Iine Natural Gas Sales by Interstate Pipeline Companies, 1987

(Describes and analyzes direce sales to end users by interstale pipuline companies and SIC eodes.)

Summary of An Lxamination of Domestic (ias Resource Estimates

(Provides an overview of the various matural gas resource cestimates currently in use.)

\section{May 1989}

The Outlook for Natural Gas Supply in the 1990's

(Discusses recent conditions in the oil and gas producing industry.)

\section{June 1989}

Domestic Natural Gas Reserves and Production

Dedicated to Interstate Pipeline Companies, 1988

(Provides preliminary data on matural gass reserves and production dedicaled to the interstate matket.)

\section{August 1989}

U.S. Natural Gas Imports and L.iports - 1988

(Comains linal 1988 data on all U.S. imports and exports of malural gass.)

\section{September 1989}

Natural Cas Production in the Post-NGPA Decade

(Presents data on the production of matural gas by caltegericen defined under the Natural (ials Policy Act of I) 78 (N(IPA).)

\section{Natural Gas Wellhead Decontrol Act of 1989}

(Describes the calegories of natural gas most affected by the decontrol provisions and provides market data for these callegories and the overall markets during the 198()$\left.^{\prime} s.\right)$

\section{March 1990}

The Developing Natural Gas Futures Market and Its Potential Impact on Domestic Natural Gas Markets

(Discusses futures murkets vs, physicals markets (which inclucle spot markets.)

\section{April 1990}

The Outlook for Natural Gas

(Discusses projections of natural gas markets through the year 2010.)

\section{May 1990}

Domestic Natural Gas Reserves and Production Dedicated to Interstate Pipeline Companies, 1989

(Provides preliminary data on natural gas reserves and production dedicaled to the interstale market.)

\section{June 1990}

\section{U.S. Natural Gas Imports and Exports - 1989}

(Comtains final 1989 ) data on all U.S. imports and exports of matural gats.)

\section{July 1990}

Resurgence in Natural Gas Wellhead Productivity

(Explores the fictors that entributed to the recent increases in matural gas wellhead production.) 
Appendix E

\section{Technical}

Contacts 


\section{Appendix E}

\section{Technical Contacts}

\section{Siction)}

Summary Statistis:s:

Natural Cias Produclion

and Comsumption

Findaction $1.0 \mathrm{ss}$

Supplemental Gaserous fuels

Imports and Exports

Price:

Cily Gate, Residential,

commercial. and Industrial

Imports and Jurchases

from Produceres

Wellhead

EActric Unliu

NCil'A Calcerri:

prices And Volumes

Summary of Natural Cias Imports and Exports

Preducer Related Activitits

Natural Ciat Preducluem

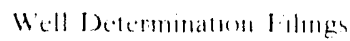

NGPA Colling prices

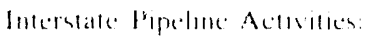

Tables

1, 2, 3 Monilily:

Allital:

Monthly

Monthly:

Annual:

Momilily:

Amolual:

2

Monthly

Alinual:

4

4

5

h

(uarlity

7

Momilily

r. 9. 11 .

11

Mumblily

M(m)tils

$15,1+$, Importers and J sporemen of Natural

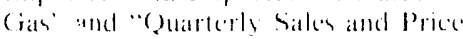
Repurit"

Principal Data Sources

Technical comtact

Interstate ( )il Compact (ommission (IO)

Form l:1A-627, "Annual Quanlily" and Value of Nalural (ias Reporl"

Form IAA-857, "Mombly Repure of Natural (ias Purchases and Deliveries (1) Cinsumets"

EiA compulations

lorm bIA-S16, "Mombly Nalural

(ias liquids Reporl" and Form IIA-64A. "Ammal Report of lhe (Origin of Natural (iats liquaids production"

Fis computatioms

Form RaA-176, "Annual Reporl of Narural and Supplemental (jas Sup) ply and Disposition"

EIA computations

Form Flec - 14, "Anmual Reporl for lmporters and I ixporters of Nalural (ials"

Furm İIA $857, " M$ onthly Report of Natural Gats Purchates and I) Coliveries io Comsumers"

Furm IV:RC-11, "Natural (iats Pipelime Compans Mombly Slatement"

JIA c(mII)UIalloms

Form lis -6.27, "Annual Q (uantity and Valuc of Natural (ias Reporl"

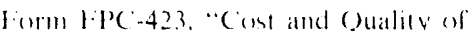

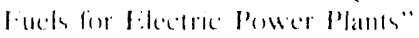

l'urchiased (ias Adjustment liblinger

Interstale ()il (impate (immisstull (I) $(C)$

form 1.1:R( $-121, "$ Applisatom for

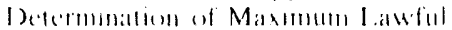

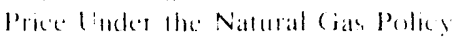
Al: $\cdot$

lederal Renastar

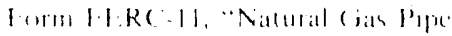

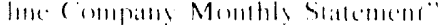

Sheila Dandell

(202) $5806-6165$

Margon Nallof

(20)2) $580-6.3(13$

Margon Nalol

(2(12) $58(2-6.3(1) .3$

Margo Natef (2012) $586-0.3013$

Fay Dillard

(2(12) $580-6181$

Roy Kass

(20) $5 \times(3.4790$

James kecling (2012) $580-610107$

Sheilat Darnell (201) $580(1-6) 65$

Mareno Natel (202) $58(1-6.303$

Nolman (rablece (2)12) 58,06180)

Jamen Tordano (2(1) $5 x(3-6.3) 5$

Sheila Darmall (20) $5 \times(206165$

Nomman ( Tablree (2012) $58(3.1) 180)$

I.val temmeng (201) >xonotii:

Janture Kictinge (201?) $58(1-6) 107$ 
17. 18, 19,

20, 21

Distribution and Consumption:

Deliveries 10:

Residential,

Commerciul,

Industrial,

Electric Unilits,

All Consumers

Average Price 10

City Gatto

Residential.

Commercial.

lndustrial.

Electric Utility,

and All Consumers

Onsystem Sales

Heating Degree Days

Highlights and Industry

Overview

Recent Developments
Monthly: . Forms FERC-8 and LIA-19), "Underground Gas Storage Report"

Monthly:

Form EIA-857. "Monthly Report of" Natural Gas Purchases and Deliveries 10 Consumers"

Form FERC-423. "Cost and Quality of Fucels for Electric Power Plants"

Monthly:

Form EIA-857, "Monthly Report of Natural Gan Purchases and Deliverices to Consumers"

Form FERC-423, "Cost and Quality" of Fuels for Electric Power Plants"

Monthly:

Form ElA-857, "Momthly Report of Natural Gas Purchase's and Deliveries to Consumers"

Seasonal: National Oceanic and Almospheric

Administration
JUlis Maupin (2(12) $58(2-6) 178$

Roy Kass

(20) $588(1-4700$

Margon Nallof

(20) $58(0-6.30 .3$

Roy Kass

(202) $5880-4780$

Margo Natof

(2()2) $58(6-6.30) 3$

Roy Kass

(2()) $586-4700$

Rosemary fameson

(20) $586-6229$

Jamen Todare (2012) $586(-6.305$

James Todaro (20) $5 \times(1-6.305$ 


\section{Glossary}

Base (Cushion) Gas: The volume of gas needed ats a permanent inventory 10 maintain adequate underground storage reservoir pressures and deliverability rates throughout the withdrawal season. All native gas is included in the base gas volume.

British Thermal Init (Btu): The heal required fo raise the termperature of one pound of water by one degree Fahrenheit at or near 39.2 degrees Fahrenheit.

City-gate: A point or measuring station at which a gat distribution company receives gas from a pipeline company or transmission system.

Commercial Consumption: Gas used by nommanufacturing organizations such as hotels, restaurants, retail stores, laundries, and other service enterprises, and gat; used by local, State, and Federal agencies engaged in nommanufacturing activities.

Depletion: The loss in service value incurred in connection with the exhaustion of the natural gas reserves in the course of service.

Depreciation: The loss in service value not restored by current maintenance, incurred in connection with the consumption or respective retirement of a gas plant in the course of service from causes that are known to be in current operation and against which the utility is not protected by insurance: for example. wear and tear, decay, obsolescence, changes in demand and requirements of public authorities, and the exhaustion of natural resources.

Dry Natural Gas Production: Marketed production less extraction loss.

Hectric Utility Consumption: Gas used as fuct in electric utility plants.
Exports: Natural gas deliveries out of the Continental United States (including Alaska) fo foreign countries. $\{, 3\}$

Extraction Loss: The reduction in volume of natural gas resulting from the renoval of natural gats liquid constituents al natural gats processing plants.

Flared: The volume of gas burned in flares on the base site or at gas processing plants.

Gross Withdrawals: Full well stream volume, including all natural gals plant liquid and nonhydrocarbon gases, but excluding lease condensate. Also includes amounts delivered as royally payments or consumed in field operations.

Hinshaw Pipeline: A pipeline or local distribution company that has received exemption, (by Section 1 (c) of the Natural Gas Act), from regulations pursuant to the Natural Gas Act. These companies transport interstate natural gas not subject to regulations under NGA.

Imports: Natural gas received in Ho Contincental United States (including Alaska) from a forcign country.

Independent Producers: Independent natural gas producers subject to the jurisdiction of the laRC:

Industrial Consumption: Natural gits used by manufacturing and mining establishments for heal, power. and chemical feedstexk.

Interstate Companies: Natural gas pipeline companies subject or FERC jurisdicion. 
Intransit Deliveries: Redeliveries to a foreign country of foreign gas received for transportation across U.S. territory and deliveries of U.S. gas to a foreign country for transportation across its territory and redelivery to the United States.

Intransit Receipts: Receipts of foreign gas for transportation across U.S. territory and redelivery to a foreign country and redeliveries to the United States of U.S. gas transported across foreign territory.

Intrastate Companies: Companies not subject to FERC jurisdiction.

Lease and Plant Fuel: Natural gas used in lease operatıons, as gas processing plant fuel, and net used for gas lift.

Liquefied Natural Gas (LNG): Natural gas that has been liquefied by reducing its temperature to minus 260) degrees Fahrenheit at atmospheric pressure.

Marketed Production: Gross withdrawals less gas used for repressuring. quantities vented and flared. and nonhydrocarbon gases removed in treating or processing operations. Includes all quantities of gas used in field and processing operations. See Explanatory Note 1 for discussion of coverage of data concerning nonhydrocarbon gases removed.

Major Interstate Pipeline Company: A company whose combined sales for resale, and gas transported interstate or stored for a fee, exceeded 50 million thousand cubic feet in the previous year.

Native Gas: Cias in place at the lime that a reservoir was converted to use as an underground storage reservoir. Excludes quantities of gas added or injected.

Natural Gas: A mixture of hydrocarbon compounds and small quantities of various nonhydrocarbons existing in the gaseous phase or solution with oil in natural underground reservoirs at reservoir conditions.

Natural (ias Policy Act of 1978 (NGPA): Signed into law on November 9, 1978, the NGPA is a framework for the regulation of most facets of the natural gas industry. See Explanatory Note lo for a full discussion.

Nonhydrocarbon Gases: Typical nonhydrocarbon gases that may be present in reservoir natural gas are carbon dioxide, helium, hydrogen sulfide, and nitrogen.

Onsystem Sales: Sales to customers where the delivery point is a point on, or directly intercomnected with, a transportation, storage, and/or distribution system operated by the reporting company.

Pipeline Fuel: Gas consumed in the operation of pipelines, primarily in compressors.

Repressuring: The injection of gas info oil or gas formations for pressure maintenance and cycling purposes.

Residential Consumption: Gas used in private dwellings, including apartments, for heating, cookung, water healing, and other household uses.

Storage Additions: The volume of gas injected or otherwise added to underground natural gas or liquefied natural gas storage during the applicable reporting period.

Storage Withdrawals: Total volume of gas withdrawn from underground storage or liquefied natural gas storage during the applicable reporting period.

Supplemental Gaseous Fuels Supplies: Synthetic natural gas, propane-air, refinery gas, biomass gas, air injected for stabilization of heating content, and manufactured gas commingled and distributed with natural gas.

Synthetic Natural Gas (SNG): A manufactured product chemically similar in most respects to natural gas, that results from the conversion or reforming of petroleum hydorcarbons and may easily be substituted for or interchanged with pipeline quality natural gas.

Therm: One-hundred thousand British thermal units. 
Unaccounted For: Represents the difference belween the sum of the components of natural gats supply and the sum of the components of natural gas dispositiont. These differences may be due to quantities lost or to the effects of data reporting problems. Reporting problems include differences due to the net result of conversions of flow data metered at varying temperature and pressure bases and converted to a standard $1 \mathrm{~cm}$ perature and pressure base: the effect of variations in company accounting and billing practices; differences between hilling cecele and calcendar period time frames; and imbalances resulting from the merger of data reporting systems which vary in scope, format, definitions, and type of respondents.

Underground Gas Storage Reservoir Capacity: Interstate company reservoir capacities are those certificated by FERC Independent producer and intrastate company reservoir capacities are reported an developed capacity.
Vented Gas: Gas released into the air on the base site or at processing plants.

Wellhead Price: Represents the wellhead sales price, including charges for natural gas plant liquids subsequently removed from the gas, gathering and compresson charges, and State production, severance, an's/or similar charges.

Working (Top Storage) (Gas: The volume of gat in an underground storage reservoir above the designed level of the base. It may or may not be completely withdraw'n during any particular withdrawal season. Conditions permitting, the total working capacity could be used more than once during any season. 


\section{NATURAL GAS MENTHLY}

3. Froqueney of lesue

MONTHLY
$9 / 25 / 90$

36. Annual Subscription Pitce DOMESTIC $\$ 70.00$ IOREIGN $\$ 87.50$

1000 Independence Ave. SW

washington, DC 20585

U.S. Department of Energy ava priwer)

nergy Information Administration

1000 Independence Ave. SW

U.S. Department of Energy Wropington, DG 20585

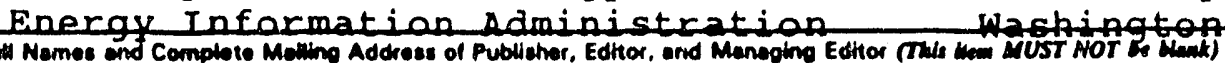

Publisher avame and Compher Molline Nidress)

U.S. Department of Energy

Energy Information Administration

1000 Independence Ave. SW Washington, DC 20585

Editor (Nane and Complese Mailine Address)

DOROTHY PRICHETT

U.S. Department of Energy

1000 Independence Ave. SW

Managing Editor arlome and Complace Malline Addrest

Washington. 20585

Patricia Jacobus

1000 Independence Ave. SW

U.S. Department of Energy

washington, DC 20585

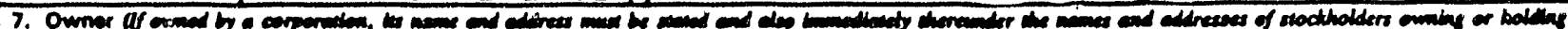

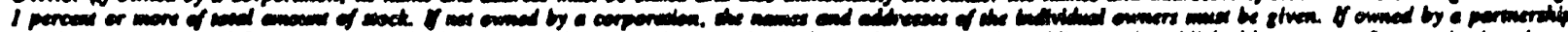

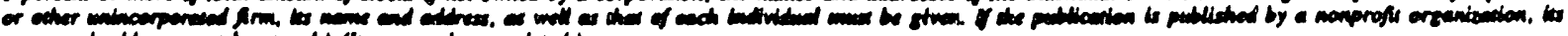

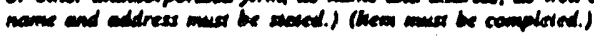

\begin{tabular}{|c|c|}
\hline Fun Nome & Complote Malling Addrees \\
\hline & \\
\hline & \\
\hline & \\
\hline & \\
\hline & \\
\hline
\end{tabular}

8. Known Bondholders, Mortgeceess. end Other Securtty Malders Owning or Holding 1 Percemt or Mere of Totel Amount of Bonds, Mortoeges of Other Socurities (of dhere ane nome, do sare)

\begin{tabular}{|c|c|c|}
\hline Ful Name & Complote Malling Addrese \\
\hline & \\
\hline & \\
\hline & \\
\hline$N / A$ & \\
\hline & \\
\hline
\end{tabular}

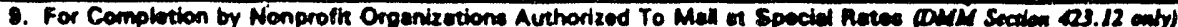

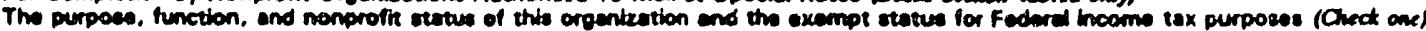

(1)

Has Not Chenged During

1. Preceding 12 Monthe
(2)

$\square^{\text {Has Chenoed During }}$

Freceding 12 Momth

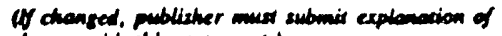
charge will shis enorement.)

\begin{tabular}{|c|c|c|c|}
\hline 10. & Exram end Anture of Ciravetion & Awereos No. Coptes Eech lewi Durino & 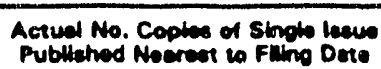 \\
\hline \multicolumn{2}{|c|}{ 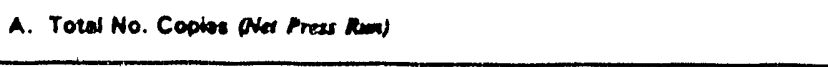 } & 1569 & 1579 \\
\hline \multicolumn{2}{|c|}{ 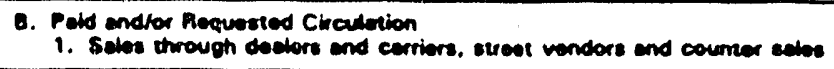 } & 142 & 142 \\
\hline \multicolumn{2}{|c|}{ 2. Moll subocription } & 1333 & 1343 \\
\hline \multicolumn{2}{|c|}{ 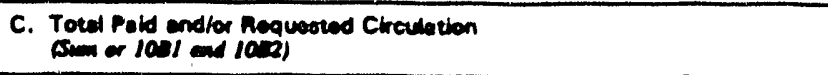 } & 1475 & 1485 \\
\hline \multicolumn{2}{|c|}{ 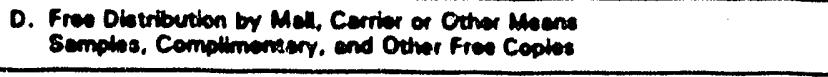 } & 0 & 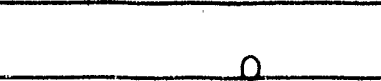 \\
\hline \multicolumn{2}{|c|}{ 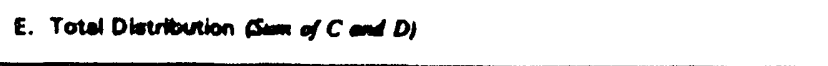 } & 1475 & 1485 \\
\hline \multicolumn{2}{|c|}{ 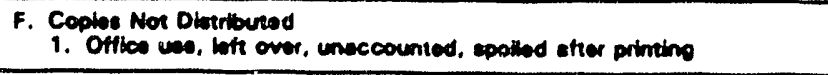 } & 34 & 94 \\
\hline \multicolumn{2}{|c|}{ 2. Rotum from Nows Agoms } & $\Omega$ & مـ \\
\hline \multicolumn{2}{|c|}{ 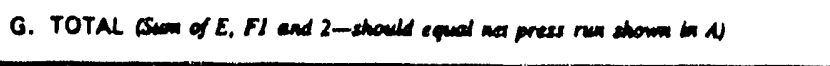 } & i559 & 7579 \\
\hline \multicolumn{2}{|c|}{$\begin{array}{l}\text { 11. I certify that the statements made by } \\
\text { me above are correct and complete }\end{array}$} & $\begin{array}{l}\text { Trob of Editor, Publiaher. Busingse Mon } \\
\text { Analy st. }\end{array}$ & our. pr Owng \\
\hline
\end{tabular}




\section{NOTICE}

\section{U.S.C. 1722 Provldes as follows:}

Whoever knowingly submits to the Postal Service or to any officer on omployee of the Postal Service, any false evidence relative to any publication for the purpose of securing the admiselon thereof at the Second-Class rate. for transportation in the malls, shall be fined mot more than $\$ 500$.

\section{INSTRUCTIONS TO PUBLISHERS}

1. Complete and file one copy of this form with your post. master on or betore October 1. A copy of the compluted form should be retained for your records.

2. Include in iterns 7 and 8 , page 1 , in cases where the slockholder or security holder appears upon the books of the compeny as trustes or in any other fiduciery ralation. the name of the person or corporation for whom euch trustee is acting. Also, include in items 7 and 8 the nemes and uddresses of individuals who are stockholders of the corporation which itself is a stockholder or holder of bonds. mortegeges or other securities of the publishing corporation when the interests of such individuats are equivalent to 1 percent or more of the sotal emoumt of the stock or securities of the publishing corporation.
3. Be sure to furnish all information called for in item 10 , page 1, regarding circulation. Show requested circulation in hem 10.B. and $C$. for requestor (DMM section 423.4 I publications ONLY. Fres circulation of other second-cless publications must be shown in item 10.0.

\section{Item 11 must be signed.}

5. If the publication has second-class entry under the provistons of DMM soction 423.1 or 423.4, the Statement of Ownership, Management and Circulation must be pub. Mahod; it must be printed in the second issue rearest to the date form 3526 is flled.

\section{INSTRUCTIONS TO POSTMASTERS}

1. Furnish each publisher not less than 10 days prior to October 1 at bast 2 copies of Form 3526 for each of his publications having en original second-class entry at your office.

2. Examine each statement to see that hecomteins all of the information required by law.

3. Verity that the known office of publication is at addreas indicated in item 4, pago 1 (DMM section 422.3).

4. Compere the information furnished in item 1082 with informution furnishod on applicable Form 3541. Statement of Mailing-2nd-Class Pubs Except Requester Publications (DMM 463), or Form 3541-A, Stetement of MallingSecond-Class/Requester Publications (DMM 463), if publication has no additional entry offices. If the pubtication has additional entry offices, compere information in hem 1082 with the findings of your verification of the publisher's records (DMM soction 425.4). Report discrepancies to the Rates and Classification Center (RCC) BSee DMM 1321.
5. Return incermplete or incorrect statements to the publishers and obtain trom them complete and correct stetomants.

6. The comphated form MUST be retained at your office.

7. Obtein a copy of the issus of each publication in which the required atetement is pultiached, and verify the correctness of the publistred statement. File the copy. DO NOT forwerd $k$ to the RCC. Promptly report to the RCC eny instance whore - publisher falls to publish a statemom. if required.

8. Indicate in blocks below informetion concerning the conditions of second-class omtry shown on second-class euthorization on fite at your office.

9. Vertty then a form 3526 is fibed for every second-cless publication which has its office of original entry at your office.

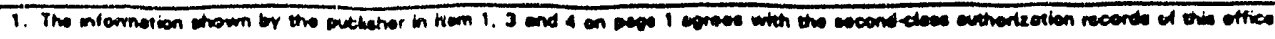

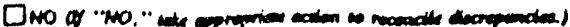

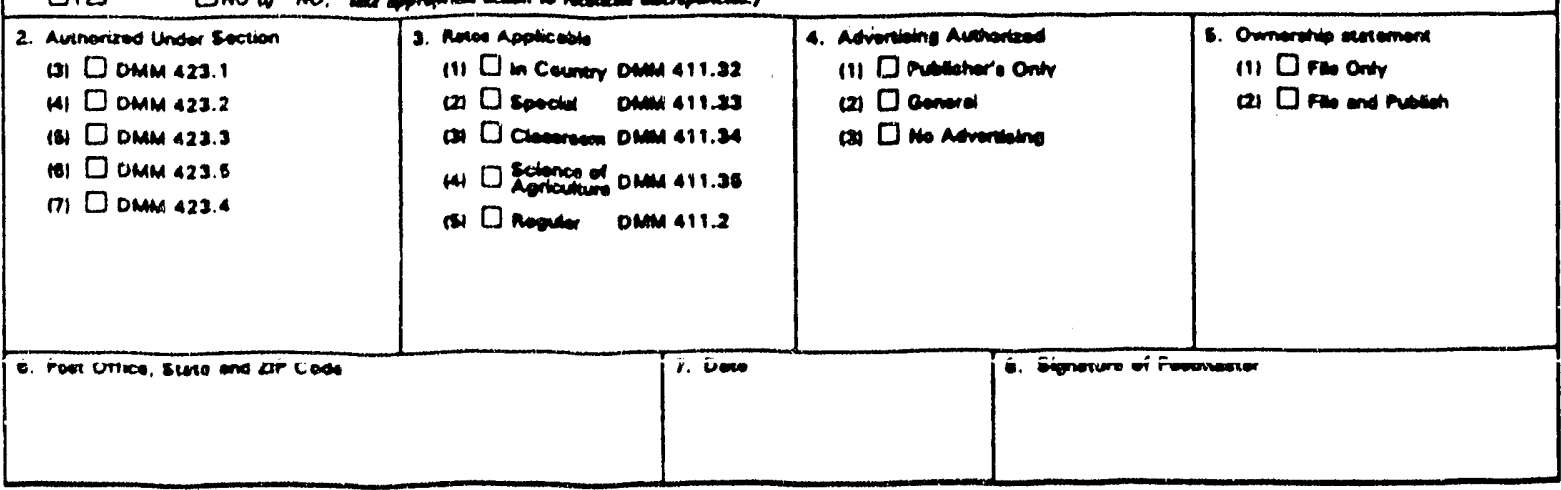



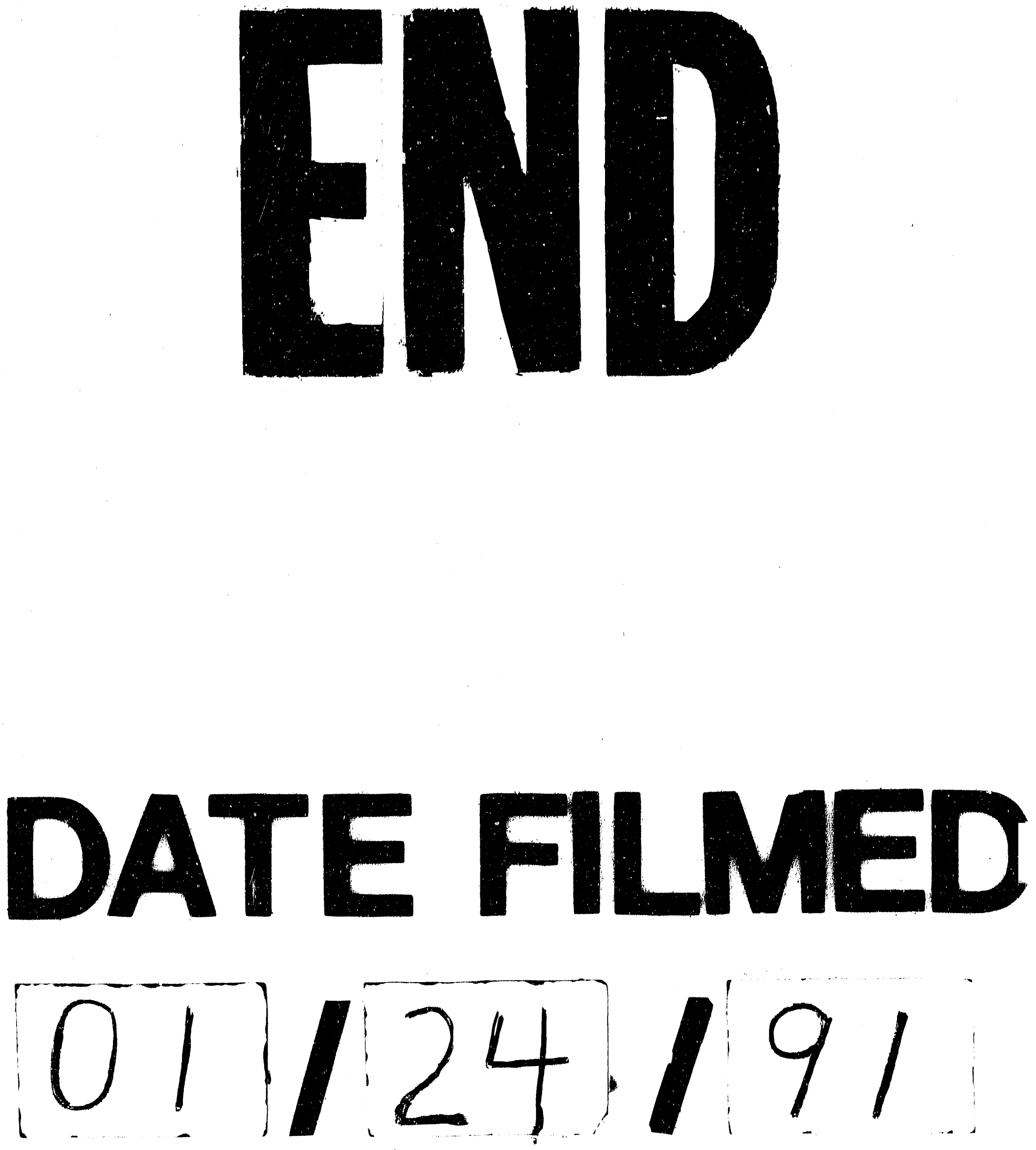
